\title{
\begin{tabular}{l|l} 
Mibraries & DSpace@MIT
\end{tabular}
}

\author{
MIT Open Access Articles
}

MASSES, RADII, AND ORBITS OF SMALL KEPLER PLANETS:
THE TRANSITION FROM GASEOUS TO ROCKY PLANETS

The MIT Faculty has made this article openly available. Please share how this access benefits you. Your story matters.

Citation: Marcy, Geoffrey W., Howard Isaacson, Andrew W. Howard, Jason F. Rowe, Jon M. Jenkins, Stephen T. Bryson, David W. Latham, et al. “MASSES, RADII, AND ORBITS OF SMALL KEPLER PLANETS: THE TRANSITION FROM GASEOUS TO ROCKY PLANETS." The Astrophysical Journal Supplement Series 210, no. 2 (January 13, 2014): 20. (C 2014 The American Astronomical Society

As Published: http://dx.doi.org/10.1088/0067-0049/210/2/20

Publisher: IOP Publishing

Persistent URL: http://hdl.handle.net/1721.1/92945

Version: Final published version: final published article, as it appeared in a journal, conference proceedings, or other formally published context

Terms of Use: Article is made available in accordance with the publisher's policy and may be subject to US copyright law. Please refer to the publisher's site for terms of use. 


\section{MASSES, RADII, AND ORBITS OF SMALL KEPLER PLANETS: THE TRANSITION FROM GASEOUS TO ROCKY PLANETS*}

Geoffrey W. Marcy ${ }^{1}$, Howard IsaACSON ${ }^{1}$, Andrew W. Howard ${ }^{2}$, Jason F. Rowe ${ }^{3}$, Jon M. Jenkins ${ }^{4}$, Stephen T. Bryson ${ }^{3}$, David W. Latham ${ }^{5}$, Steve B. Howell ${ }^{3}$, Thomas N. Gautier III $^{6}$, Natalie M. Batalha ${ }^{3}$, Leslie Rogers ${ }^{7}$, David Ciardi ${ }^{8}$,

Debra A. Fischer ${ }^{9}$, Ronald L. Gilliland ${ }^{10}$, Hans KJeldsen ${ }^{11}$, Jørgen Christensen-Dalsgaard ${ }^{11,12}$, Daniel Huber ${ }^{3}$, William J. Chaplin ${ }^{11,13}$, Sarbani Basu ${ }^{9}$, Lars A. Buchhave ${ }^{5,14}$, Samuel N. QuinN ${ }^{5}$, William J. Borucki ${ }^{3}$, David G. Koch ${ }^{3}$, Roger Hunter ${ }^{3}$, Douglas A. Caldwell ${ }^{4}$, Jeffrey Van Cleve $^{4}$, Rea Kolbl ${ }^{1}$, Lauren M. Weiss ${ }^{1}$, Erik Petigura ${ }^{1}$, Sara Seager $^{15}$, Timothy Morton ${ }^{7}$, John Asher Johnson ${ }^{7}$, Sarah Ballard ${ }^{16}$, Chris Burke ${ }^{4}$, William D. Cochran ${ }^{17}$, Michael Endl ${ }^{17}$, Phillip MacQueEn ${ }^{17}$, Mark E. Everett ${ }^{18}$, JACK J. Lissauer ${ }^{3}$, Eric B. Ford ${ }^{19}$, Guillermo Torres ${ }^{5}$, Francois Fressin ${ }^{5}$, Timothy M. Brown ${ }^{20}$, Jason H. StefFen ${ }^{21}$, David Charbonnead ${ }^{5}$, Gibor S. Basri ${ }^{1}$, Dimitar D. Sasselov ${ }^{5}$, Joshua WinN ${ }^{15}$, Roberto Sanchis-Ojeda ${ }^{15}$, Jessie Christiansen ${ }^{3}$, Elisabeth Adams ${ }^{22}$, Christopher Henze ${ }^{3}$, Andrea Dupree ${ }^{5}$, Daniel C. Fabrycky ${ }^{23}$, Jonathan J. Fortney ${ }^{24}$, Jill Tarter ${ }^{4}$, Matthew J. Holman $^{5}$, Peter Tenenbaum ${ }^{4}$, Avi Shporer ${ }^{7}$, Philip W. Lucas ${ }^{25}$, William F. Welsh ${ }^{26}$, Jerome A. Orosz ${ }^{26}$,

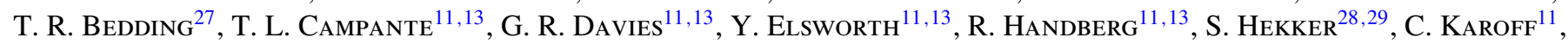
S. D. Kawaler ${ }^{30}$, M. N. Lund ${ }^{11}$, M. Lundkvist ${ }^{11}$, T. S. Metcalfe ${ }^{31}$, A. Miglio ${ }^{11,13}$, V. Silva Aguirre ${ }^{11}$, D. Stello ${ }^{27}$, T. R. White ${ }^{27}$, Alan Boss ${ }^{32}$, Edna Devore ${ }^{4}$, Alan Gould ${ }^{33}$, Andrej Prsa ${ }^{34}$, Eric Agol ${ }^{16}$, Thomas Barclay ${ }^{35}$,

Jeff Coughlin $^{35}$, Erik Brugamyer ${ }^{36}$, Fergal Mullally ${ }^{4}$, Elisa V. Quintana ${ }^{4}$, Martin Still ${ }^{35}$, Susan E. Thompson ${ }^{4}$, David Morrison ${ }^{3}$, Joseph D. Twicken ${ }^{4}$, Jean-Michel DéserT ${ }^{5}$, Josh CARTer ${ }^{15}$, Justin R. Crepp ${ }^{37}$, Guillaume Hébrard ${ }^{38,39}$, Alexandre Santerne ${ }^{40,41}$, Claire Moutou ${ }^{42}$, Charlie Sobeck ${ }^{3}$, Douglas Hudgins ${ }^{43}$, Michael R. Haas ${ }^{3}$, Paul Robertson ${ }^{17,19}$, Jorge Lillo-Box ${ }^{44}$, and David Barrado ${ }^{44}$

${ }^{1}$ University of California, Berkeley, CA 94720 , USA

${ }^{2}$ University of Hawaii, Honolulu, HI, USA

${ }^{3}$ NASA Ames Research Center, Moffett Field, CA 94035, USA

${ }^{4}$ SETI Institute/NASA Ames Research Center, Moffett Field, CA 94035, USA

${ }^{5}$ Harvard-Smithsonian Center for Astrophysics, 60 Garden Street, Cambridge, MA 02138, USA

${ }^{6}$ Jet Propulsion Laboratory/Caltech, Pasadena, CA 91109, USA

${ }^{7}$ California Institute of Technology, Pasadena, CA 91109, USA

${ }^{8}$ NASA Exoplanet Science Institute/Caltech, Pasadena, CA 91125, USA

${ }^{9}$ Yale University, New Haven, CT 06510, USA

${ }^{10}$ Center for Exoplanets and Habitable Worlds, The Pennsylvania State University, University Park, 16802, USA

${ }^{11}$ Stellar Astrophysics Centre (SAC), Department of Physics and Astronomy, Aarhus University, Ny Munkegade 120, DK-8000 Aarhus C, Denmark

${ }^{12}$ High Altitude Observatory, National Center for Atmospheric Research, Boulder, CO 80307, USA

${ }^{13}$ School of Physics and Astronomy, University of Birmingham, Edgbastron, Birmingham B15 2TT, UK

${ }^{14}$ Centre for Star and Planet Formation, Natural History Museum of Denmark, University of Copenhagen, DK-1350 Copenhagen, Denmark

${ }^{15}$ Massachusetts Institute of Technology, Cambridge, MA 02139, USA

${ }^{16}$ Department of Astronomy, Box 351580, University of Washington, Seattle, WA 98195, USA

${ }^{17}$ University of Texas, Austin, TX 78712, USA

${ }^{18}$ NOAO, Tucson, AZ 85719, USA

${ }^{19}$ Center for Exoplanets and Habitable Worlds, Department of Astronomy and Astrophysics, 525 Davey Laboratory,

The Pennsylvania State University, University Park, PA 16802, USA

${ }^{20}$ Las Cumbres Observatory Global Telescope, Goleta, CA 93117, USA

${ }^{21}$ Northwestern University, Evanston, IL 60208, USA

22 Planetary Science Institute, 1700 East Fort Lowell, Suite 106, Tucson, AZ 85719, USA

${ }^{23}$ Department of Astronomy and Astrophysics, University of Chicago, 5640 South Ellis Avenue, Chicago, IL 60637, USA

${ }^{24}$ University of California, Santa Cruz, CA 95064, USA

${ }^{25}$ Centre for Astrophysics Research, University of Hertfordshire, College Lane, Hatfield, AL10 9AB, UK

${ }^{26}$ San Diego State University, San Diego, CA 92182, USA

${ }^{27}$ Sydney Institute for Astronomy, School of Physics, University of Sydney 2006, Australia

${ }^{28}$ Max-Planck-Institut für Sonnensystemforschung, D-37191 Katlenburg-Lindau, Germany

${ }^{29}$ Astronomical Institute, "Anton Pannekoek," University of Amsterdam, The Netherlands

${ }^{30}$ Department of Physics and Astronomy, Iowa State University, Ames, IA 50011, USA

${ }^{31}$ Space Science Institute, Boulder, CO 80301, USA

${ }^{32}$ Carnegie Institution of Washington, Department of Terrestrial Magnetism, Washington, DC 20015, USA

${ }^{33}$ Lawrence Hall of Science, Berkeley, CA 94720, USA

${ }^{34}$ Villanova University, Dept. of Astronomy and Astrophysics, 800 East Lancaster Avenue, Villanova, PA 19085, USA

${ }_{35}$ Bay Area Environmental Research Institute/Moffett Field, CA 94035, USA

${ }^{36}$ McDonald Observatory, University of Texas at Austin, Austin, TX 78712, USA

${ }^{37}$ University of Notre Dame, Notre Dame, Indiana 46556, USA

${ }^{38}$ Institut d'Astrophysique de Paris, UMR7095 CNRS, Université Pierre and Marie Curie, 98bis boulevard Arago, F-75014 Paris, France

39 Observatoire de Haute Provence, CNRS/OAMP, F-04870 Saint-Michel-l'Observatoire, France

${ }^{40}$ Aix Marseille Université, CNRS, LAM UMR 7326, F-13388 Marseille, France

${ }^{41}$ Centro de Astrofisica, Universidade do Porto, Rua das Estrelas, 4150-762 Porto, Portugal

${ }^{42}$ Canada-France-Hawaii Telescope, 65-1238 Mamalahoa Hwy, Kamuela, HI 96743, USA

${ }^{43}$ NASA Headquarters, Washginton DC, USA

${ }^{44}$ Depto. Astrofísica, Centro de Astrobiología (INTA-CSIC), ESAC campus, P.O. Box 78, E-28691 Villanueva de la Cañada, Spain Received 2013 October 28; accepted 2013 December 12; published 2014 January 13 


\section{ABSTRACT}

We report on the masses, sizes, and orbits of the planets orbiting 22 Kepler stars. There are 49 planet candidates around these stars, including 42 detected through transits and 7 revealed by precise Doppler measurements of the host stars. Based on an analysis of the Kepler brightness measurements, along with high-resolution imaging and spectroscopy, Doppler spectroscopy, and (for 11 stars) asteroseismology, we establish low false-positive probabilities (FPPs) for all of the transiting planets (41 of 42 have an FPP under 1\%), and we constrain their sizes and masses. Most of the transiting planets are smaller than three times the size of Earth. For 16 planets, the Doppler signal was securely detected, providing a direct measurement of the planet's mass. For the other 26 planets we provide either marginal mass measurements or upper limits to their masses and densities; in many cases we can rule out a rocky composition. We identify six planets with densities above $5 \mathrm{~g} \mathrm{~cm}^{-3}$, suggesting a mostly rocky interior for them. Indeed, the only planets that are compatible with a purely rocky composition are smaller than $\sim 2 R_{\oplus}$. Larger planets evidently contain a larger fraction of low-density material $\left(\mathrm{H}, \mathrm{He}\right.$, and $\left.\mathrm{H}_{2} \mathrm{O}\right)$.

Key words: planetary systems - planets and satellites: detection - stars: individual (Kepler-25, Kepler-37, Kepler-48, Kepler-68, Kepler-93, Kepler-94, Kepler-95, Kepler-96, Kepler-97, Kepler-98, Kepler-99, Kepler-100, Kepler-102, Kepler-103, Kepler-106, Kepler-109, Kepler-113, Kepler-131, Kepler-406, Kepler-407, Kepler-409) techniques: photometric - techniques: radial velocities

Online-only material: color figures, machine-readable table

\section{INTRODUCTION}

Our solar system contains no planets with radii between those of Earth and Neptune $\left(3.9 R_{\oplus}\right)$, a size gap that differs from the apparent distribution of small planets in the Milky Way and requires adjustments to the core-accretion model to explain. For example, Uranus and Neptune, with equatorial radii of 4.01 and $3.88 R_{\oplus}$, respectively, and their massive rocky cores would presumably have grown to Saturn or even Jupiter size through runaway accretion had the protoplanetary disk not disappeared when it did (Pollack et al. 1996; Goldreich et al. 2004; Rogers \& Seager 2010b; Morbidelli 2013). Still, based on formation models of our own solar system, the size domain of $1-4 R_{\oplus}$ was expected to be nearly deserted (Ida \& Lin 2010; Mordasini et al. 2012). It is not. Instead, most of the observed planets around other stars have radii in the range of $1-4 R_{\oplus}$ (Borucki et al. 2011; Batalha et al. 2013).

This great population of sub-Neptune-size exoplanets had first been revealed by precise Doppler surveys of solar-mass stars within $50 \mathrm{pc}$. Such surveys find planet counts increase toward smaller masses, at least within the range of $1000 M_{\oplus}$ down to $\sim 5 M_{\oplus}$ (Howard et al. 2010; Mayor et al. 2011). Independently, the NASA Kepler telescope finds that $85 \%$ of its transiting planet "candidates" have radii less than $4 R_{\oplus}$ (Batalha et al. 2013). Since more than $80 \%$ of these small planet candidates are actually planets (Morton \& Johnson 2011; Fressin et al. 2013), the population of sub-4- $R_{\oplus}$ planets is assuredly large (but see Santerne et al. (2012) for the confirmation rate of Jupiter-size planets). No detection bias would favor the discovery of small planets over the large ones (for a given orbital period), and indeed the small planets enjoy a smaller rate of false-positive scenarios. Thus, in both the solar vicinity probed by Doppler surveys and in the Kepler field of view (slightly above the plane of the Milky Way), an overwhelming majority of planets orbiting within 1 au of solar-type stars are smaller than Uranus and Neptune (i.e., $<\sim 4 R_{\oplus}$ ).

For planets orbiting close to their host star, the great occurrence of small planets is particularly well determined. Within 0.25 au of solar-type stars, the number of planets rises rapidly

\footnotetext{
* Based in part on observations obtained at the W. M. Keck Observatory, which is operated by the University of California and the California Institute of Technology.
}

moving from $15 R_{\oplus}$ to $2 R_{\oplus}$, based on analyses of Kepler data that correct for detection biases due to photometric noise, orbital inclination, and the completeness of the Kepler planet-search detection pipeline (Howard et al. 2012; Fressin et al. 2013; Petigura et al. 2013). Further corrections for photometric signal-to-noise ratio $(\mathrm{S} / \mathrm{N})$ and detection completeness show that the occurrence of planets remains at a (high) constant level for sizes from 2 to $1 R_{\oplus}$, with $\sim 15 \%$ of FGK stars having a planet of $1-3 R_{\oplus}$ within 0.25 au (Fressin et al. 2013; Petigura et al. 2013).

With no solar system analogs, the chemical compositions, interior structures, and formation processes for $1-4 R_{\oplus}$ planets, including their gravitational interactions with other planets, present profound questions (Seager et al. 2007; Fortney et al. 2007; Zeng \& Seager 2008; Rogers et al. 2011; Zeng \& Sasselov 2013; Lissauer et al. 2011, 2012; Fabrycky et al. 2012). Determining chemical composition is one step toward a deeper understanding, but at this planet-size scale, the relative amounts of rock, water, and $\mathrm{H}$ and $\mathrm{He}$ gas remain poorly known. Most likely, the admixture of those three ingredients changes as a function of planet mass, but differs among planets at a given mass, as well.

Beyond the question of their characteristics, these $1-4 R_{\oplus}$ planets pose a great challenge for the theory of planet formation: like Venus, Earth, Uranus, and Neptune, they likely contain a ratio of rock to light material that is much greater than cosmic abundances, and therefore their formation must have required some complex processing in the protoplanetary disk. However, new ideas are emerging about the formation of such Neptune-mass-and-smaller planets, most of which are variations on the theme of core-accretion theory (Chiang \& Laughlin 2013; Mordasini et al. 2012; Hansen \& Murray 2013). Particularly intriguing is the notion that taken as an ensemble, the hundreds of Kepler exoplanet candidates reflect the mass densities of protoplanetary disks during the period of planet formation, leading to a theory that within 0.5 au of their host stars, sub-Neptunes formed in situ, i.e., without migration (Chiang \& Laughlin 2013; Hansen \& Murray 2013). The predicted relations between mass, radius, and incident flux agree with those observed (Lopez et al. 2012; Lopez \& Fortney 2013; Weiss et al. 2013). These models and their associated predictions of in situ mini-Neptune and super-Earth formation can be further tested with accurate measurements of planet masses and radii. 
Measuring masses for transiting planets that already have measured radii can constrain the mean molecular weight, internal chemical composition, and hence formation mechanisms for 1-4 $R_{\oplus}$ planets (Seager et al. 2007; Zeng \& Seager 2008; Zeng \& Sasselov 2013; Rogers et al. 2011; Chiang \& Laughlin 2013). Only a handful of small planets have mass measurements, and those with radii above $2 R_{\oplus}$ often have low densities inconsistent with pure rocky composition. Two well-studied examples are GJ 436 b and GJ 1214 b (Maness et al. 2007; Gillon et al. 2007; Torres et al. 2008; Charbonneau et al. 2009) with radii of 4.21 and $2.68 R_{\oplus}$, masses of 23.2 and $6.55 M_{\oplus}$, and resulting bulk densities of 1.69 and $1.87 \mathrm{~g} \mathrm{~cm}^{-3}$, respectively. Their densities are slightly higher than those of Uranus and Neptune (1.27 and $\left.1.63 \mathrm{~g} \mathrm{~cm}^{-3}\right)$, but still well below Earth's $\left(5.5 \mathrm{~g} \mathrm{~cm}^{-3}\right)$. The sub-Earth bulk densities indicate that the two exoplanets contain significant amounts of low-density material (by volume), presumably H, He, and water (Figueira et al. 2009; Rogers \& Seager 2010a; Batygin \& Stevenson 2013). At the larger end of the small-planet spectrum is Kepler-18 c, with a radius of $5.5 R_{\oplus}$ but a mass of only $17.3 M_{\oplus}$, implying a low density of $0.59 \pm 0.07 \mathrm{~g} \mathrm{~cm}^{-3}$ (Cochran et al. 2011). Similarly, HAT-P-26, with $6.3 R_{\oplus}$, has low density of $0.40 \pm 0.1 \mathrm{~g} \mathrm{~cm}^{-3}$ (Hartman et al. 2011).

The several other $\sim 2-4 R_{\oplus}$ exoplanets with secure masses and radii support this trend, including the five inner planets around Kepler-11, GJ 3470 b, 55 Cnc e, and Kepler-68 b (Lissauer et al. 2013; Bonfils et al. 2012; Endl et al. 2012; Demory et al. 2013, 2011; Gilliland et al. 2013). All of these planets have densities less than $5 \mathrm{~g} \mathrm{~cm}^{-3}$ and some under $1 \mathrm{~g} \mathrm{~cm}^{-3}$, indicating a significant amount of light material by volume $(\mathrm{H}, \mathrm{He}$, water) mixed with some rock and Fe. (The uncertainties for $55 \mathrm{Cnc}$ e admit the possibility this $2.1 R_{\oplus}$ planet could be pure rock.) Perhaps these securely measured lower-than-rock densities are representative of planets of size $2.0-4.5 R_{\oplus}$ in general, and hence representative of the chemical composition of such planets.

Most tellingly, the five planets with radii less $2 R_{\oplus}$, namely CoRoT 7b, Kepler-10b, Kepler-36b, KOI-1843.03, and Kepler$78 \mathrm{~b}$ all have measured densities of $6-10 \mathrm{~g} \mathrm{~cm}^{-3}$ (Queloz et al. 2009; Batalha et al. 2011; Carter et al. 2012; Rappaport et al. 2013; Sanchis-Ojeda et al. 2013; Pepe et al. 2013; Howard et al. 2013). Thus, below $2 R_{\oplus}$ some planets have densities consistent with pure solid rock and iron-nickel. The dichotomy of planet densities has been considered theoretically as due to accumulation and photo-evaporation of volatiles (Chiang \& Laughlin 2013; Hansen \& Murray 2013; Lopez et al. 2012; Lopez \& Fortney 2013).

To quantify this transition to rocky planets, one may use the extant empirical relation between density and planet mass that has been discovered for the planets smaller than $5 R_{\oplus}$ : $\rho=1.3 M_{p}^{-0.60} F^{-0.09}$, where $\rho$ is in $\mathrm{g} \mathrm{cm}^{-3}, M_{p}$ is in $M_{\oplus}$, and $F$ is the incident stellar flux on the planet in erg s $\mathrm{s}^{-1} \mathrm{~cm}^{-2}$ (Weiss et al. 2013). The Weiss et al. relation shows that planets with masses over $\sim 2 M_{\oplus}$ (equivalently, with radii over $1.5 R_{\oplus}$ ) have typical densities less than $5.5 \mathrm{~g} \mathrm{~cm}^{-3}$ and hence typically contain significant amounts of light material ( $\mathrm{H}, \mathrm{He}$, and water). Thus, the transition from planets containing significant light material to those that are rocky occurs at planet radii near $1.0-2.5 R_{\oplus}$, i.e., masses near $1-3 M_{\oplus}$, based tentatively on the handful of planets in that size domain located within $0.2 \mathrm{au}$. This suggestion of a transition to rocky planets below masses of $3 M_{\oplus}$ is a major result from current Kepler exoplanet observations.
However, the Weiss et al. relation, and the predicted transition to rocky planets below $2 R_{\oplus}$, is based on the measured masses and radii of only a handful of planets. It surely requires both confirmation and quantification, by measuring the masses and radii of more small exoplanets. Those additional small exoplanets would also greatly inform models of planet formation, based on correlations between the volatile or rocky nature of the planets and the metallicities of their host stars (Buchhave et al. 2012; Latham \& Buchhave 2012; Johnson et al. 2007).

Here we report measured masses, radii, and densities (or upper limits on those values) for 42 transiting planet candidates contained within 22 bright Kepler Objects of Interest (KOIs) from Batalha et al. (2013). We carried out multiple Dopplershift measurements of the host stars using the Keck 1 telescope. From the spectroscopy and Doppler measurements, we compute self-consistent measurements of stellar and planet radii, employing either stellar structure models or asteroseismology measurements from the Kepler photometry. We also search for (and report) 7 additional non-transiting planets revealed by the precise radial velocities (RVs), for a total of 49 planets.

\section{VETTING AND SELECTION OF 22 TARGET KOIs}

This paper contains the results of extensive precise-RV measurements of KOIs, made by the Kepler team. The intense RV follow-up observations described here were carried out on 22 KOIs chosen through a careful vetting process. The initial identification of the KOIs from the photometry was an extensive, iterative program carried out by the Kepler team during the nominal NASA mission from launch 2009 March to 2012 November. The identification process has been described elsewhere, notably by Caldwell et al. (2010), Jenkins et al. (2010b, 2010a), Van Cleve \& Caldwell (2009), and Argabright et al. (2008), with an overview in Borucki et al. (2010). The $\sim 2300$ KOIs identified in these searches are listed in Batalha et al. (2013).

\subsection{Data Validation: TCERT}

The selection of the 22 KOIs for this study involved several major stages of pruning of the candidates, starting with the "threshold crossing events" (TCEs) that are the series of repeated dimmings found for a particular star by the Kepler "Transit Planet Search" pipeline. Working within the Kepler TCE review team ("TCERT"), we vetted the TCEs to distinguish planet candidates from false positives and to measure more accurately the properties of the planets and their host stars. Detailed descriptions of the components of this TCE vetting can be found in Gautier et al. (2010), Borucki et al. (2011), and Batalha et al. (2013).

A TCE was elevated to KOI status (planet candidate) based on simple (often eye-ball) criteria involving the inspection of each Kepler light curve, using long cadence photometry, overplotted on a model of a transiting planet, and noting a lack of eclipsing binary signatures such as secondary eclipses and "odd-even" alternate variability of successive transit depths. This TCERTbased identification of the KOIs involved only Kepler data, not outside observations.

Actual transiting planets should exhibit photometry that is well fit, within errors, by a transiting planet model. They should also show an astrometric displacement (if any) during transit that is consistent with the hypothesis that the intended target star is the source of the photometric variations during transit. Such "data validation" (DV) techniques are described in Batalha 
et al. (2010, 2011) and Bryson et al. (2013). These DV tests have undergone improvements and automation during the past 3 yr (Wu et al. 2010; Bryson et al. 2013). All 22 KOIs in this work passed their DV tests, conferring KOI status on them as continued planet candidates. Details on the nature of DV criteria for each KOI are given below in Section 7.

The TCERT identification of KOIs as "planet candidates" made them worthy of follow-up observations with other telescopes, designed both to weed out false positives and to better measure the planet properties through superior knowledge of host star properties, notably radii. Various types of follow-up observations of some, but not all, of the $\sim 2300$ KOIs had been carried out by the time of our selection process of the $22 \mathrm{KOIs}$ studied here. Publication of those KOIs are in Borucki et al. (2011, 2012) and Batalha et al. (2013).

\subsection{Follow-up Observation Program: KFOP}

We activated in 2009 May the Kepler "Follow-up Observation Program" (KFOP) with the goals of vetting the KOIs for false positives and improving the measurement of the planet radii. The goals were to characterize all of the KOIs, as resources permitted, using a variety of ground-based telescopes. Each of the KOIs first had their Kepler light curves and astrometric integrity scrutinized again, polishing the TCERT vetting. Here we summarize the key KFOP observational efforts that were carried out on $\sim 1000 \mathrm{KOIs}$ from which the $22 \mathrm{KOIs}$ presented here were selected.

In brief, each of the $\sim 1000$ KOIs had its light curve further scrutinized and its position further measured (Section 2.1) to alert us to angularly nearby stars (within $2^{\prime \prime}$ ) in the photometric aperture. As described below, we carried out adaptive optics (AO) imaging and speckle interferometry (Sections 2.2.3 and 2.2.2) to hunt for neighboring stars. KOIs having a neighboring star within $2^{\prime \prime}$ and brighter than $1 \%$ of the primary star are not amenable for follow-up spectroscopy due to the light from both stars entering the slit. Roughly $20 \%$ of the KOIs were deemed not suitable for spectroscopy due a close stellar neighbor.

KOIs meeting those criteria were observed with highresolution, low-S/N echelle spectroscopy to measure atmospheric stellar parameters, magnetic activity, and rotational Doppler broadening, designed to detect binaries and, importantly, to assess suitability for precise RV measurements. Only single, FGKM-type stars with narrow lines $\left(v \sin i<10 \mathrm{~km} \mathrm{~s}^{-1}\right)$ are suitable for the highest precision RV measurements. Below is a summary of the nature of these KFOP vetting actions on over $1000 \mathrm{KOIs}$, prioritized by brightness, leading to the selection of the $22 \mathrm{KOIs}$ in this study. KOIs brighter than Kp $\sim 14.5 \mathrm{mag}$ received most of the KFOP observational resources, while those fainter than $K p=15$ mag were only rarely observed.

\subsubsection{Follow-up Reconnaissance Spectroscopy}

We carried out "reconnaissance" high-resolution spectroscopy on $\sim 1000$ KOIs with spectral resolution, $R \sim 50,000$, and $\mathrm{S} / \mathrm{N}=20-100$ per pixel. The dual goals were searching for false positives and refining the stellar parameters. We obtained one or two such reconnaissance spectra using one of four facilities: the McDonald Observatory $2.7 \mathrm{~m}$, the Tillinghast $1.5 \mathrm{~m}$ on Mt. Hopkins, the Lick Observatory $3 \mathrm{~m}$, and the $2.6 \mathrm{~m}$ Nordic Optical Telescope.

Of greatest importance was to detect angularly nearby stars that, themselves, might be eclipsed or transited by a companion star or planet, the light from which would be diluted by the primary star mimicking a transiting planet around it. With a typical spectrometer slit width of $1^{\prime \prime}$, stellar companions within 0 '.5 would send light into the slit, permitting their detection if bright enough (see below). A cross-correlation of each spectrum was performed, usually with a best-matched synthetic template, to detect stellar companions separated by more than $\sim 10 \mathrm{~km} \mathrm{~s}^{-1}$ in RV and brighter than $\sim 5 \%$ of the primary in optical flux.

Also, a second reconnaissance spectrum was obtained to detect RV variation above a threshold of $\sim 0.5 \mathrm{~km} \mathrm{~s}^{-1}$, indicating the presence of a binary. We selected the 22 KOIs in this paper by rejecting all KOIs that showed such RV variation from binary motion. The fraction of KOIs rejected by reconnaissance spectroscopy was roughly $5 \%$, leaving $95 \%$ as surviving planet candidates. The absence of a secondary spectrum and RV variations (confirmed later by the precise RVs with $2 \mathrm{~m} \mathrm{~s}^{-1}$ precision) for all $22 \mathrm{KOIs}$ rules out a large portion of parameter space for possible false positives in the form of an angularly nearby star that may be the source of the periodic dimming. As described in Section 6, a further analysis of the Keck-HIRES spectra taken later with $\mathrm{S} / \mathrm{N}$ further ruled out stellar companions within 0.5 down to optical flux levels of $1 \%$ that of the primary star.

The reconnaissance spectra were also analyzed to measure the properties of the host star more precisely than was available in the Kepler Input Catalog (KIC). The spectra were analyzed by comparing each one to a library of theoretical stellar spectra, e.g., Buchhave et al. (2012). This "recon" analysis (later refined by Buchhave et al. as "SPC" analysis) was done with grid step sizes between individual library spectra of $250 \mathrm{~K}$ for $T_{\text {eff }}$, 0.5 dex for $\log g, 1 \mathrm{~km} \mathrm{~s}^{-1}$ for $v \sin i$, and 0.5 dex in metallicity $([\mathrm{m} / \mathrm{H}])$. This "recon" spectroscopy analysis yielded approximate values of $T_{\text {eff }}$ (within $200 \mathrm{~K}$ ), $\log g$ (within $0.10 \mathrm{dex}$ ), and $v \sin i$ (within $2 \mathrm{~km} \mathrm{~s}^{-1}$ ) for the primary star of the KOI, valuable for deciding whether the KOI was suitable for followup precise RV observations. Only stars cooler than $6100 \mathrm{~K}$ on the main sequence $(\log g>4.0)$ with $v \sin i<5 \mathrm{~km} \mathrm{~s}^{-1}$ were deemed suitable for the RV measurements of highest precision near $\sim 2 \mathrm{~m} \mathrm{~s}^{-1}$. All relevant details about the reconnaissance spectroscopy for each KOI are given in Section 7.

\subsubsection{Speckle Imaging}

Speckle imaging of each of the 22 KOIs was obtained using the two-color DSSI speckle camera at the WIYN $3.5 \mathrm{~m}$ telescope on Kitt Peak, with technical details given in Howell et al. (2011) and Horch et al. (2009). The speckle camera simultaneously obtained 3000 images of $40 \mathrm{msec}$ duration in two filters: $V$ $(5620 / 400 \AA)$ and $R(6920 / 400 \AA)$. These data yielded a final speckle image for each filter. Section 7 describes the results of the speckle observation for each KOI noting if any other sources appeared.

The speckle data for each star allowed detection of a companion star within the $2.76 \times 2$.'76 field of view centered on the target. The speckle observations could detect, or rule out, companions between 0 '.05 and 1".5 from each KOI. The speckle images were all obtained with the WIYN telescope during seeing of $0.6-1{ }^{\prime \prime} .0$. The threshold for detection of companion stars was a delta magnitude of $3.8 \mathrm{mag}$ in the $R$ band and $4.1 \mathrm{mag}$ in $V$ band (within the sensitivity annulus from $00^{\prime} 05-1^{\prime \prime} .5$ ), relative to the brightness of the KOI target star. For Kepler-97 the detection threshold was compromised by a stellar companion 0.36 away from the primary and 2.7 mag fainter at optical wavelengths (3.2 mag fainter in $K$ band). This companion is farther than the 
maximum possible separation of a false positive star, based on centroid astrometry in and out of transit.

These speckle observations were used to select the $22 \mathrm{KOIs}$ studied here, by rejecting all KOIs (roughly 5\%) that showed such a stellar companion. Thus, these initial speckle observations showed that none of the 22 KOIs (except Kepler-97) in this work had a detected companion by speckle. This selection process using speckle imaging by which the 22 KOIs were chosen surely favors single stars rather than binaries or multiples. Hence it eliminates, a priori, a major domain of false positives (i.e., neighboring stars with transiting companions).

\subsubsection{AO Imaging}

Near-infrared AO imaging was obtained for $\sim 300$ KOIs of the $1000 \mathrm{KOIs}$ brighter than $K p=14 \mathrm{mag}$. The goal, as with the speckle observations described above, was to detect stellar companions that might be the source of the periodic dimming (a false positive). Seeing-limited imaging, obtained with various telescopes at both optical and IR wavelengths, revealed companions located more than $2^{\prime \prime}$ from the primary KOI star. Seeing-limited $J$-band Images from UKIRT were particularly useful (Lawrence et al. 2007). (After selection of the 22 KOIs here, we also examined optical seeing-limited images from the Keck-HIRES guide camera, and we provide those images here.) Any seeing-limited images showing a companion stars within $2^{\prime \prime}$ was rejected as a useful candidate for high precision RV work, i.e., rejected for inclusion in this study. Thus all 22 KOIs in this paper were selected with a prior AO image, as well as the speckle imaging described above.

The strength of AO imaging is the ability to detect companions located between $0.05-2$. 0 of the KOI primary star with detection limits 6-8 mag fainter than the primary (depending on the telescope and AO camera). The goal was to detect angularly nearby stars, either background or gravitationally bound, that might potentially have an eclipsing companion or a transiting planet that might mimic a transiting planet around the primary star, i.e., a false positive.

Four different AO instruments were used in the near IR on four different telescopes, namely the Keck 2 telescope on Mauna Kea (NIRC2-AO), the MMT telescope on Mt. Hopkins (ARIES), the $5 \mathrm{~m}$ telescope on Mt. Palomar (PHARO), and the $3 \mathrm{~m}$ telescope at Lick Observatory (IRCAL), each described briefly below (Hayward et al. 2001; Troy \& Chanan 2003; Adams et al. 2012). After the 22 KOIs were selected based on an absence of stellar companions found with the reconnaissance $\mathrm{AO}$ observations with those four AO instruments, we carried out subsequent $\mathrm{AO}$ imaging with the Keck 2 telescope and NIRC2 camera, generally superior to the other imaging.

All 22 KOIs were observed with the Keck NIRC2-AO system (Wizinowich et al. 2004; Johansson et al. 2008). We employed a natural guide star rather than the laser guide star as the Galactic field is rich with useful 13th mag guide stars in the Kepler field. We obtained all images on two nights, 2013 June 13/14 and $14 / 15$. We used the $K^{\prime}$ filter (wavelength coverage 1.9-2.3 $\mu$ ), except for the brightest five KOIs for which we employed a narrow-band Bracket-gamma filter to avoid saturation and to achieve flatter wavefronts. On both nights the natural seeing (before AO) was $\sim 0^{\prime} .2$ (FWHM) in $K^{\prime}$ band.

For each KOI, we obtained 15 images with NIRC2-AO, employing a pattern of three dither positions (using the three best quadrants on the detector) and five exposures at each position. The images were sky-subtracted, flat-fielded, and co-added to yield a final AO image. All final Keck AO images have a point- spread function (PSF) with a FWHM of $0.05 \pm 0$. .'01, with a field of view of $2^{\prime \prime}$.

The detection thresholds from these Keck AO images for each KOI are shown in Figures 1-44. The detection thresholds uniformly yielded $5 \sigma$ detection of delta- $K^{\prime}$ magnitude $=6$ mag as close as $0^{\prime \prime} .2$ from the KOI. At $0^{\prime \prime} .4$, the detection threshold is delta- $K^{\prime}$ magnitude $=8 \mathrm{mag}$. Excellent spatial resolution and sensitivity permit the detection of a large fraction of the background stars that could mimic a transiting planet. This spatial resolution also permits detection of a significant fraction of the widely separated bound stellar companions, given the typical distances $(\sim 200 \mathrm{pc})$ to these magnitude 10-13 solar-type stars, as described in Section 6. None of the 22 KOIs showed a stellar companion within $1^{\prime \prime}$, except for Kepler-97, described below.

The AO-vetting of the 2000 KOIs was done with four smaller telescopes. The MMT ARIES camera achieves near diffraction-limited imaging, with typical PSF FWHM of 0'.25 in the $J$ band and 0.14 in the $K s$ band, yielding Strehl ratios of 0.3 in $K s$ and 0.05 in $J$ band (Adams et al. 2012). While guiding on the primary star, a set of 16 images, on a four-point dither pattern was acquired for each KOI. Full details and a description of calibration and reduction of the images is described by Adams et al. (2012)

Some KOIs were vetted with the Palomar $5 \mathrm{~m}$ "PHARO" AO camera, observed in both the $K s$ and $J$ infrared bands using a five-point dither pattern with integration times between 1.4 and $70 \mathrm{~s}$, depending on the target brightness. The AO system used the primary star itself, not a laser, to guide and correct the images, achieving a best resolution of 0.05 at $J$ and $0{ }^{\prime} .09$ in the $K s$ band, with Strehl ratios of $0.10-0.15$ in $J$ and $0.35-0.5$ in $K s$. Typical detection thresholds were $7 \mathrm{mag}$ at a separation of 0.5 and 9.3 mag at $1^{\prime \prime} .0$.

The remaining KOIs were AO-vetted with the Lick Observatory $3 \mathrm{~m}$ telescope and high-resolution camera, "IRCAL." Observations were made in Natural Guide Star mode, allowing the AO system to guide on the target star. ${ }^{45}$

This mode of observing allows stars as faint as $K p=13.5$ to be observed. Detection limits down to a delta- $J$ magnitude of $6 \mathrm{mag}$, as close as $0^{\prime \prime} 1$ are typically achieved. Background sky emission in the $J$ band is typically 16.0 mag per square arcsecond. The $K$-band background sky emission at Lick Observatory is 10.3 mag arsec ${ }^{2}$, making $K$-band observing difficult. Typically only $J$-band images were taken at Lick Observatory. ${ }^{46}$

Adams et al. (2012) provides an excellent description of AO imaging of KOIs.

The AO imaging described above with five telescopes revealed a companion star within $6^{\prime \prime}$ of nine targets: Kepler-103, 95, 109, 48, 113, 96, 131, 97, and 407. The following KOIs have no detected stellar companion within $6^{\prime \prime}:$ Kepler-100, 93, $102,94,106,25,37,68,98,99,406,408$, and 409. Of the nine KOIs with a detected companion, only one, Kepler-97, has a companion within $1^{\prime \prime}$. See Table 3 for details.

None of the nine stars having stellar companions reside angularly within the maximum exclusion radius found from astrometry in and out of transit (see Section 6.2 and Table 3). Thus we find that none of the neighboring stars can arguably be causing the dimming as a false positive. For Kepler-97, the observed neighboring star resides 0.'38 away while the exclusion

\footnotetext{
45 The Lick IRCAL AO system, built by Claire Max and James R. Graham, is described in detail at astro.berkeley.edu/ jrg/ircal/spie/ircal.html.

46 For details about the IRCAL AO system, see

mtham.ucolick.org/techdocs/instruments/.
} 

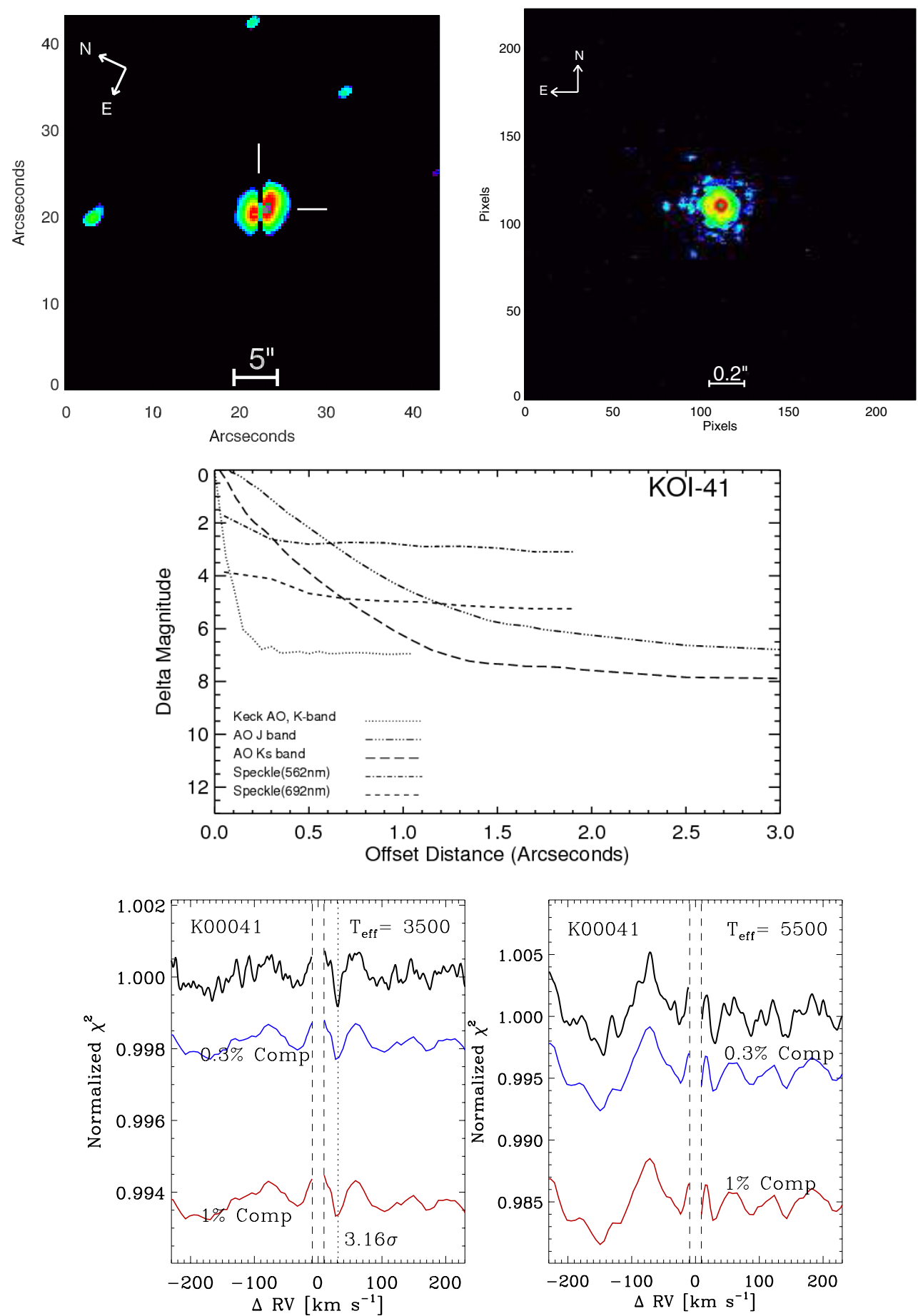

Figure 1. Upper left: seeing-limited image for Kepler-100, (KOI-41). Upper right: adaptive optics image. Middle: limiting magnitudes of companion stars found with Speckle and AO imaging. Lower left and lower right: diagnostic plots to detect the presence of absorption lines from any secondary star at a different radial velocity relative to the primary star. The plot gives the reduced chi-square statistic (black line) of the fit to the residuals (after subtracting the primary star spectrum) for representative secondary spectra for stars having $T_{\text {eff }}=3500 \mathrm{~K}$ (left panel) and $T_{\text {eff }}=5500 \mathrm{~K}$ (right panel), as a function of relative RV. Injection of such mock companion stars, at different relative RVs, was performed for a relative brightness of $0.3 \%$ (blue line) and $1 \%$ (red line). The lack of drops below those two color lines indicates the lack of companions at those thresholds. For Kepler-100, no companion star is apparent. Companions brighter than $0.3 \%$ (optical, $V$ band) would have been detected. Companions separated in velocity by less than $10 \mathrm{~km} \mathrm{~s}^{-1}$ would not be detected as the absorption lines mostly overlap.

(A color version of this figure is available in the online journal.)

radius is 0 '20, suggesting that the companion does not cause the apparent transit in the photometry.

Toward selection of the 22 KOIs in this study, any KOI with a neighboring star located within $2^{\prime \prime}$ that had more than $1 \%$ the flux of the primary star at optical wavelengths was rejected as a suitable candidate for precise RV measurements due to the contamination of light from that nearby star and due to the possible false positive. Of the $22 \mathrm{KOIs}$ in this paper, only Kepler-97 has a companion within $2^{\prime \prime}$, and it is less than $1 \%$ of the brightness of the primary star at optical wavelengths, as 

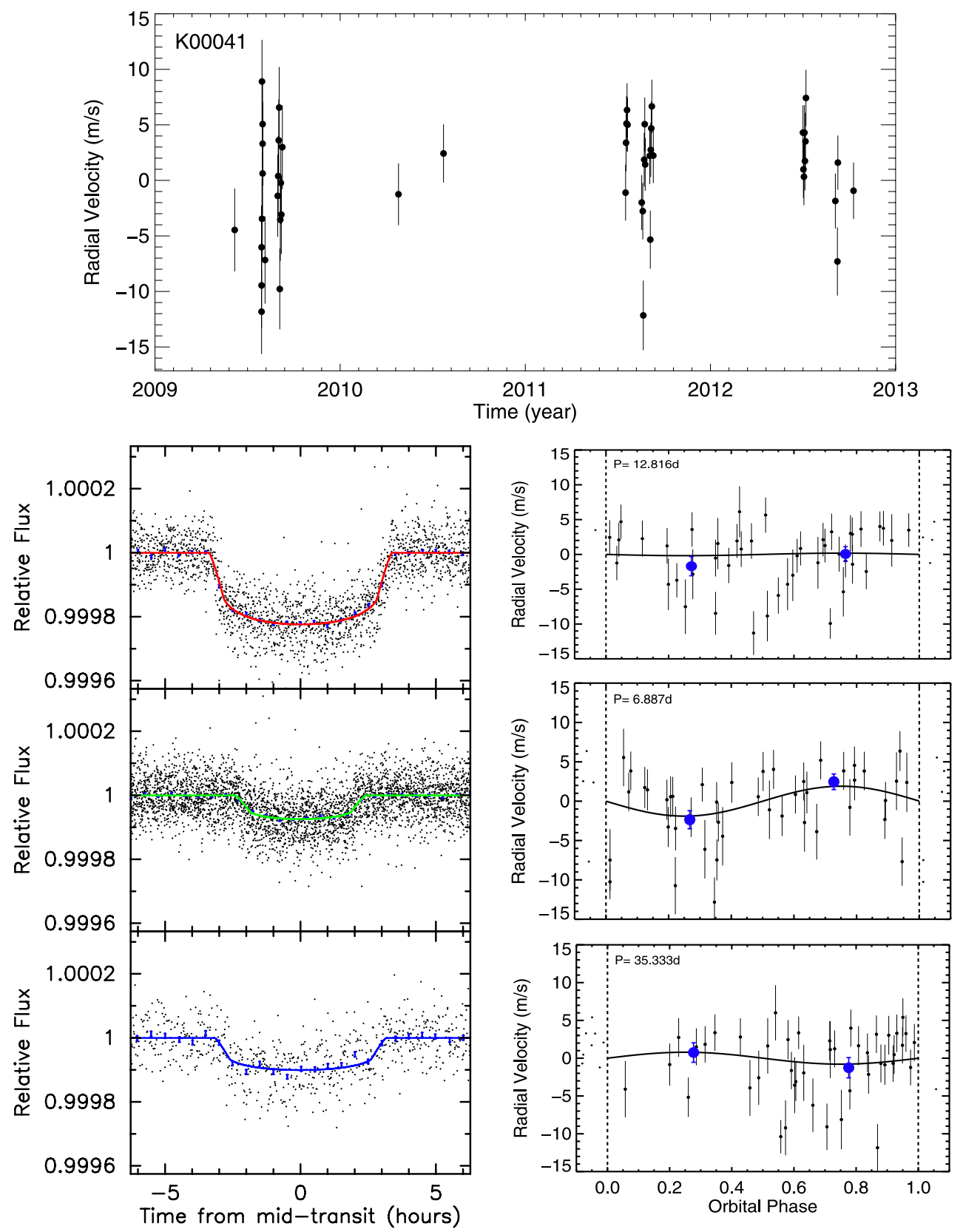

Figure 2. Top: RV vs. time for Kepler-100 (KOI-41). Lower left; phase-folded Kepler photometry for each planet. Lower right: phase-folded radial velocities for each planet. The blue points represent RVs binned into the quadrature intervals, $0.25 \pm 0.125$ and $0.75 \pm 0.125$. The amplitude of the blue point is the weighted average in each bin. The error of the blue point is the standard deviation of the RVs in the bin, divided by the square root of the number of RVs included. Kepler-100c: Rp $=$ $2.20 \pm 0.05 R_{\oplus}, \mathrm{Mp}=0.85 \pm 4.0 M_{\oplus}$. Kepler-100b: $\mathrm{Rp}=1.32 \pm 0.04 R_{\oplus}, \mathrm{Mp}=7.34 \pm 3.2 M_{\oplus}$. Kepler-100d: $\mathrm{Rp}=1.61 \pm 0.05 R_{\oplus}, \mathrm{Mp}=-4.36 \pm 4.1 M_{\oplus}$.

(A color version of this figure is available in the online journal.)

described in Section 7. The collection of imaging data and the associated search for companions, as well as the photometry and RV measurements, are found in Figures 1-44.

\subsection{Selecting the $22 \mathrm{KOIS}$}

In the last three years of the four-year Kepler mission, the TCERT committee systematically shifted its prioritization to select smaller-radii planets for precise-RV follow-up observations. Initially, the criteria had emphasized verifying the planet nature of KOIs. The effort had favored large planets with sizes above $4 R_{\oplus}$ and short-period orbits that might yield a detectable RV variation in the host star, to check the existence of Kepler transiting planets. Detections of the RV signatures of the large planets around Kepler 4, 5, 6, 7, and 8 followed from this conservative prioritization. After the successes of the first six months of the Kepler mission, the criteria shifted toward verifying and measuring the masses of the smaller planets, $2-5 R_{\oplus}$, and of planets in multi-planet systems, if they were likely to be detected with RVs. Resulting RV detections included Kepler 10, 18, 20, 22, 25 , and 68 yielding constraints on the masses of the planets.

During the second and third years of the 4 yr Kepler mission, i.e., 2010 and 2011, the TCERT prioritization shifted toward planets having smaller radii, below $3 R_{\oplus}$ and down to $1.0 R_{\oplus}$. Obviously such small planets are expected to have low masses, inducing small RV amplitudes in their host star. We carried out careful, optimized selection of suitable KOIs for RV work.

One selection criterion was a brightness limit, $K p<$ $13.5 \mathrm{mag}$, to permit Poisson-limited S/Ns near 100 per pixel 

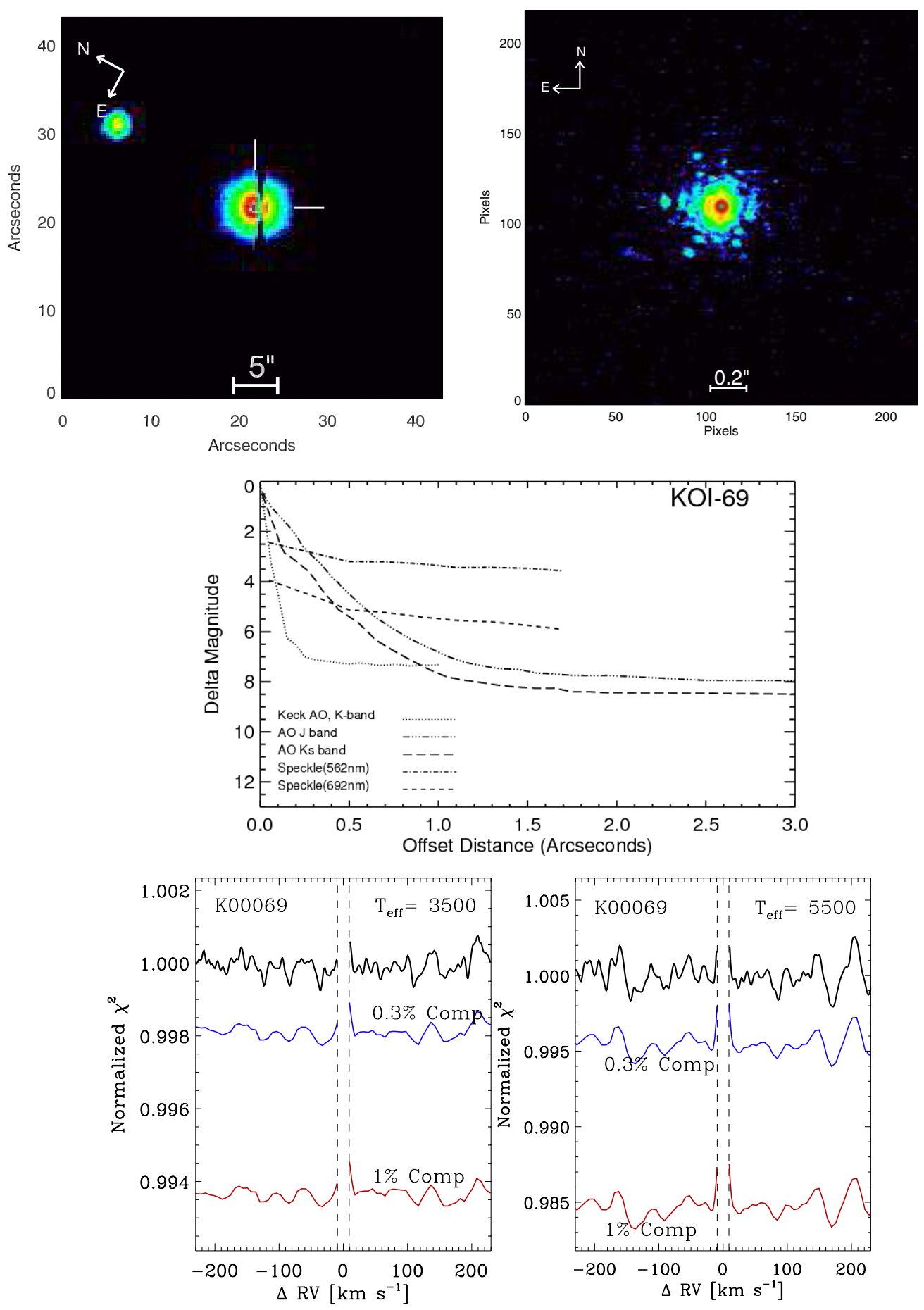

Figure 3. Upper left: seeing-limited image for Kepler-93 (KOI-69). Upper right: adaptive optics image. Middle: limiting magnitudes of companion stars found with Speckle and AO imaging. Lower left and lower right: same as Figure 1, but for Kepler-93. No companions are detected. Any companion brighter than $0.3 \%$ the brightness ( $V$ band) of the primary would have been detected.

(A color version of this figure is available in the online journal.)

within a 45 minute exposure with the Keck-HIRES spectrometer. Such exposures yield a photon-limited Doppler precision of $1.5 \mathrm{~m} \mathrm{~s}^{-1}$. Another selection criteria was $T_{\text {eff }}<6100 \mathrm{~K}$ (based on reconnaissance spectra) to promote numerous, narrow spectral lines that contribute Doppler information. Another criterion was small rotational Doppler broadening of the spectral lines, $v \sin i<5 \mathrm{~km} \mathrm{~s}^{-1}$, based on reconnaissance spectra, to limit broadening of the lines that degrades Doppler precision.
In the face of pervasive astrophysical "jitter" of $\sim 1 \mathrm{~m} \mathrm{~s}^{-1}$ for $\mathrm{G}$ and $\mathrm{K}$ dwarfs (Isaacson \& Fischer 2010), the Kepler TCERT committee selected KOIs for which an estimated planet mass might be sufficient to induce an RV amplitude greater than $1 \mathrm{~m} \mathrm{~s}^{-1}$. To anticipate the RV amplitude, we used a nominal mass based on the planet radius taken from the KIC, coupled with a rough estimate of planet density for that radius. The density assumptions were simplistically based on the planets in 

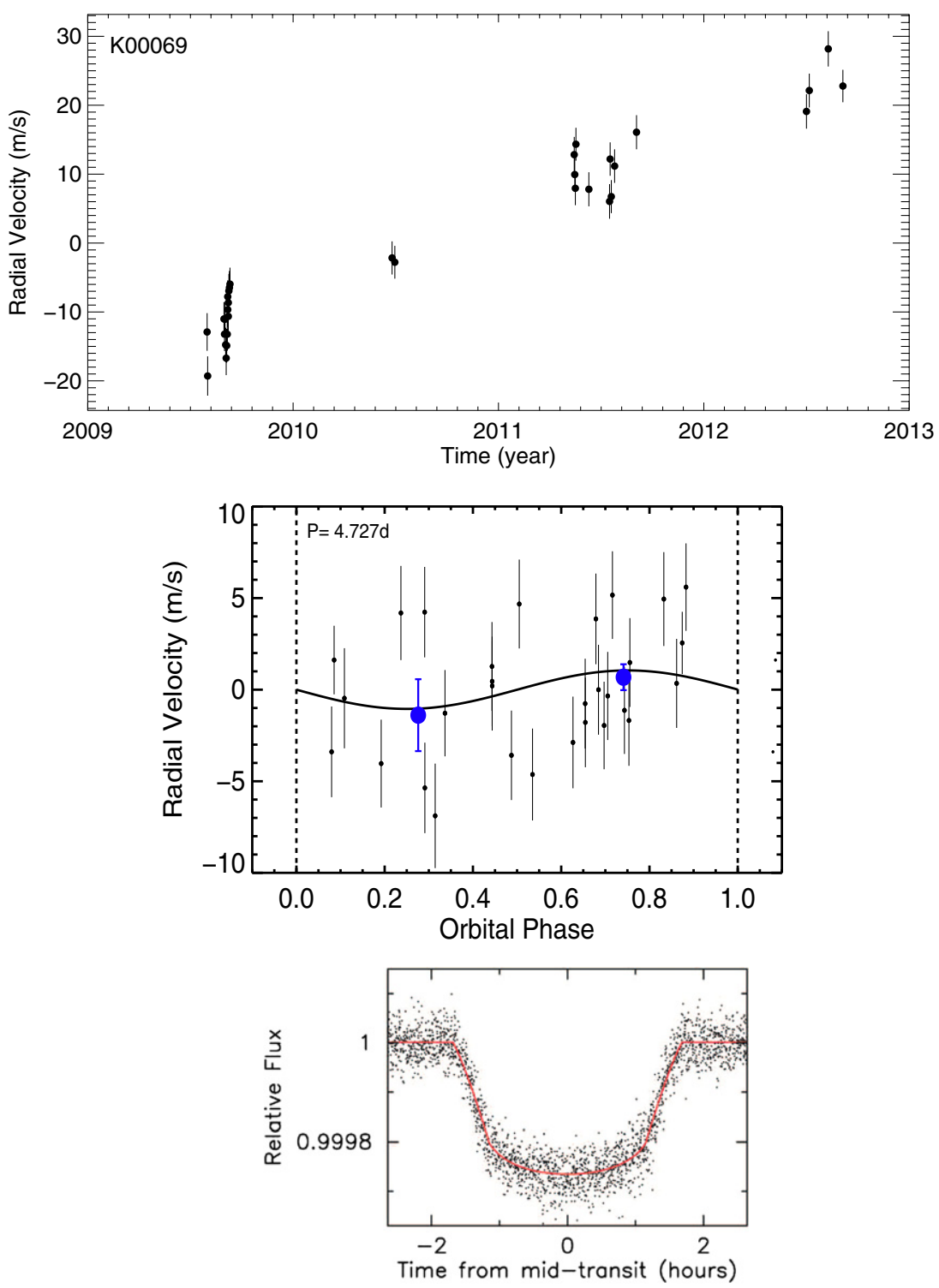

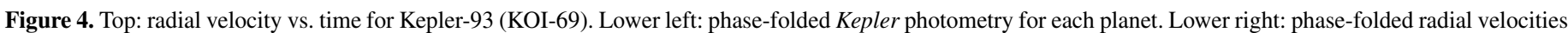

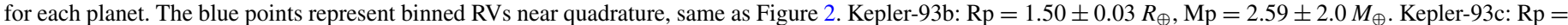
$\mathrm{NA}, \mathrm{Mp}>954 M_{\oplus}$, period $>1460$ days.

(A color version of this figure is available in the online journal.)

our solar system along with the few known small exoplanets, notably GJ436b, GJ1214b, and Kepler-10b. We simply assumed a rocky constitution and density of $\sim 5.5 \mathrm{~g} \mathrm{~cm}^{-3}$ for planets smaller than $2 R_{\oplus}$. We assumed densities of $2 \mathrm{~g} \mathrm{~cm}^{-3}$ for planets of 2-5 $R_{\oplus}$, and we assumed densities of $1 \mathrm{~g} \mathrm{~cm}^{-3}$ for planets larger than $5 R_{\oplus}$. These densities allowed the TCERT to choose planets that might meet the criteria above, including a prospective RV amplitude above $1 \mathrm{~m} \mathrm{~s}^{-1}$. The selection process was imperfect and biased as the assumed stellar parameters and planet densities were only approximately known and the target KOIs were selected based on RV detectability.

Here we report on the 22 KOIs selected by the process described above, with a preference for small planets suitable for detection and mass determination by precise RV measurements. All 22 KOIs are identified in Batalha et al. (2013), but the followup observations, their analysis, asteroseismology, and the RVs have not been published to date, except for Kepler-68 for which we provide an update to its long-period, non-transiting planet.
This sample of 22 KOIs contains neither a random selection of KOIs nor a defined distribution of any parameters. They were selected during the first $3 \mathrm{yr}$ of ever-evolving criteria, as described above.

Importantly, the planet masses were unknown at the time of target selection, except for estimates based on measured planet radii and guesses of density. Thus, for each of the selected planet candidates, the subsequently measured planet mass provides an unbiased sampling of planet masses for its particular planet radius. We could not have selected planet candidates biased toward high or low planet masses for a given planet radius, as we had no such mass indicator.

\section{STELLAR CHARACTERIZATION}

For each of the 22 KOIs, we obtained an optical "template" spectrum using the Keck telescope and HIRES echelle spectrometer (Vogt et al. 1994) with no iodine gas in the light path. 

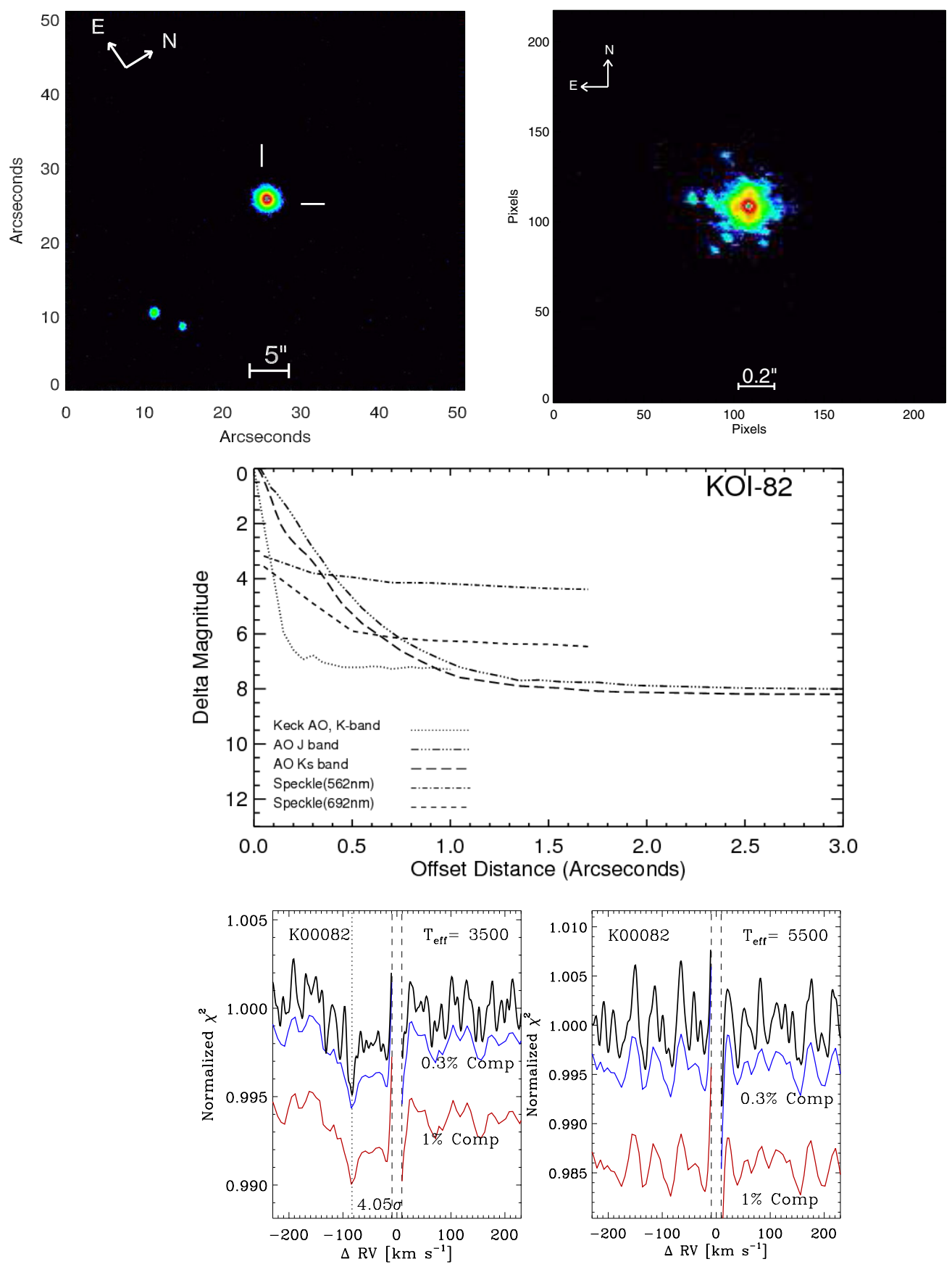

Figure 5. Upper left: seeing-limited image for Kepler-102 (KOI-82). Upper right: adaptive optics image. Middle: limiting magnitudes of companion stars found with Speckle and AO imaging. Lower left and lower right: same as Figure 1, but for Kepler-102. No companions are detected. Any companion brighter than $0.5 \%$ the brightness ( $V$ band) of the primary would have been detected.

(A color version of this figure is available in the online journal.)

Each spectrum spanned wavelengths from $3600-8000 \AA$, with a spectral resolution of $R=60,000$ and typical $\mathrm{S} / \mathrm{N}$ per pixel of 100-200. These template spectra were analyzed with the standard LTE spectrum synthesis code, SME (Valenti \& Piskunov 1996; Valenti \& Fischer 2005; Fischer \& Valenti 2005) to yield values of $T_{\text {eff }}, \log g$, and $[\mathrm{Fe} / \mathrm{H}]$ yielding formal uncertainties (of roughly $50 \mathrm{~K}, 0.1 \mathrm{dex}$, and $0.05 \mathrm{dex}$, respectively, with slight differences in precision due to $\mathrm{S} / \mathrm{N}$ and spectral type). We augment the formal uncertainties to account for addition contributions to errors seen in 56 transiting planet hosts for which constraints on stellar properties stem from analysis of the light curves (Torres et al. 2012). We added dispersions in quadrature of $\sigma_{T_{\text {eff }}}=59 \mathrm{~K}$, $\sigma_{[\mathrm{Fe} / \mathrm{H}]}=0.062$ dex. Values of $\log g$ are somewhat more uncertain and may be systematically in error for $T_{\text {eff }}>6100 \mathrm{~K}$, due to poor sensitivity of the magnesium $b$ triplet lines to surface gravity (Torres et al. 2012).

For 11 of the 22 KOIs an asteroseismic signal was detected in the Kepler photometry, namely for Kepler-100, 93, 103, 95, 109, 25, 37, 68, 406, 408 and 409.

For those 11 KOIs the output stellar parameters from the SME analysis $T_{\text {eff }}, \log g$, and [Fe/H], were fed into the asteroseismology analysis as priors. The asteroseismology analysis 

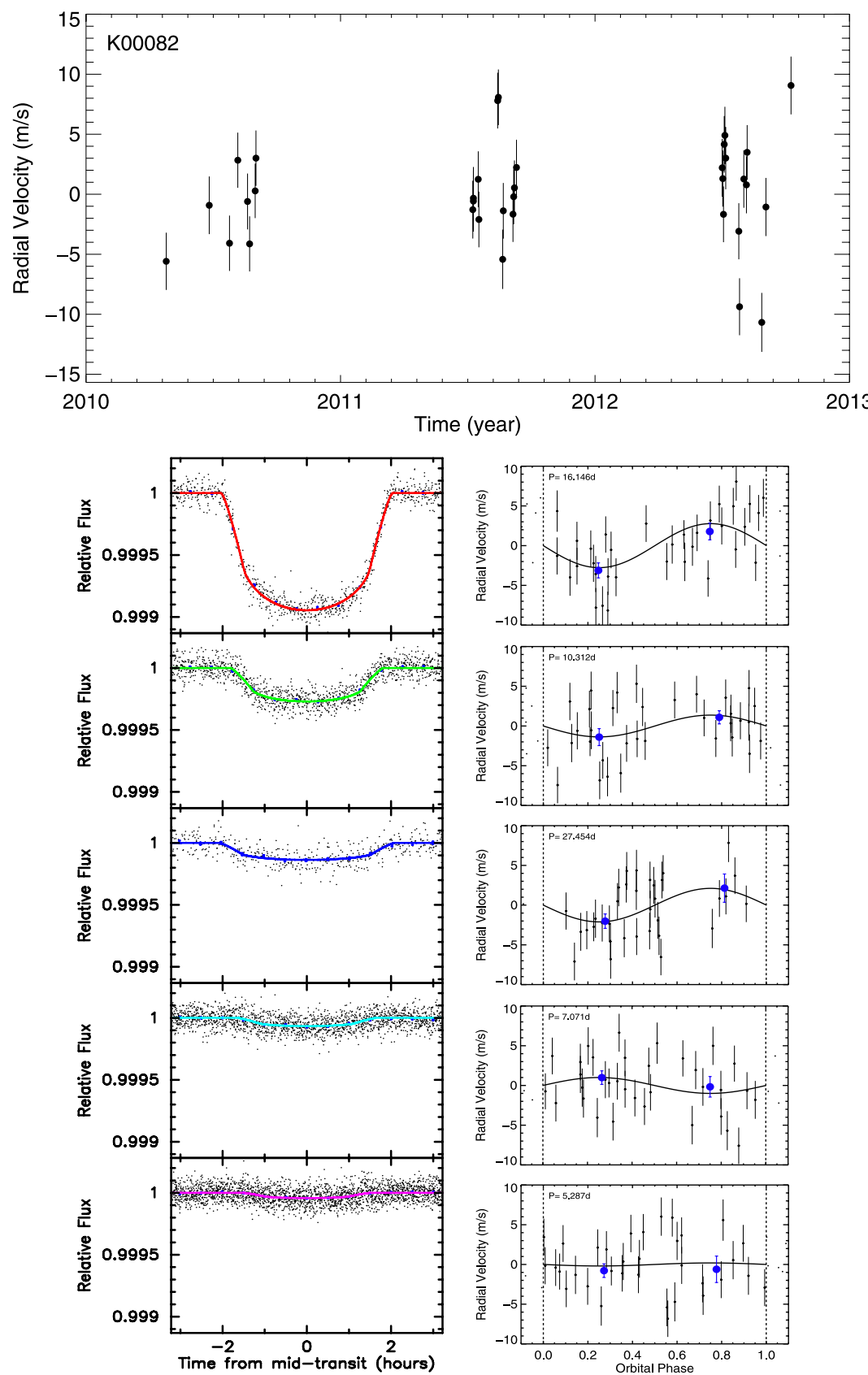

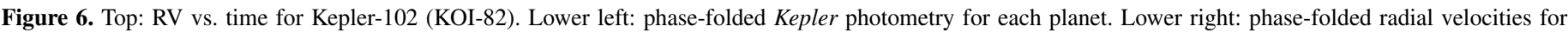

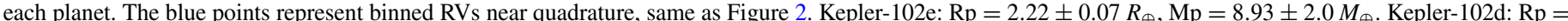

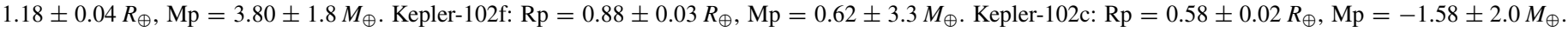
Kepler-102b: $\mathrm{Rp}=0.47 \pm 0.02 R_{\oplus}, \mathrm{Mp}=0.41 \pm 1.6 M_{\oplus}$.

(A color version of this figure is available in the online journal.)

yielded a more precise measure of stellar radius and mass, and hence of surface gravity. This surface gravity was fed back, frozen, in the SME analysis of the spectrum, allowing a redetermination of $T_{\text {eff }}$ and $[\mathrm{Fe} / \mathrm{H}]$ without the usual covariances with $\log g$. The resulting values of $T_{\text {eff }}$ and $[\mathrm{Fe} / \mathrm{H}]$ were then fed back to an asteroseismology analysis as before, achieving an iterative convergence quickly (Huber et al. 2013; Gilliland et al. 2013). The resulting uncertainties in stellar radius are between $2 \%$ and $4 \%$ (Huber et al. 2013). Stellar parameters for these $11 \mathrm{KOIs}$ with asteroseismology are reported in Table 1.

For the remaining $11 \mathrm{KOIs}$ that offered no asteroseismology signal, we determined the stellar mass and radius from the
SME spectrum analysis combined with the Yonsei-Yale stellar structure models (Yi et al. 2001; Demarque et al. 2004). The SME output values of $T_{\text {eff }}, \log g$, and [Fe/H] map to a stellar mass and radius. For the mild subgiants, the output SME stellar parameters may correspond to regions of the HR diagram where some convergence of the evolutionary tracks occurs, leaving greater uncertainties in the resulting stellar mass and radius, e.g., Batalha et al. (2011). Any such uncertainties are duly noted and included in the subsequent analysis of the properties of the planets.

The determinations of stellar masses and radii for the 22 KOIs (with or without asteroseismology) are employed as priors in a self-consistent Markov Chain Monte Carlo (MCMC) analysis 

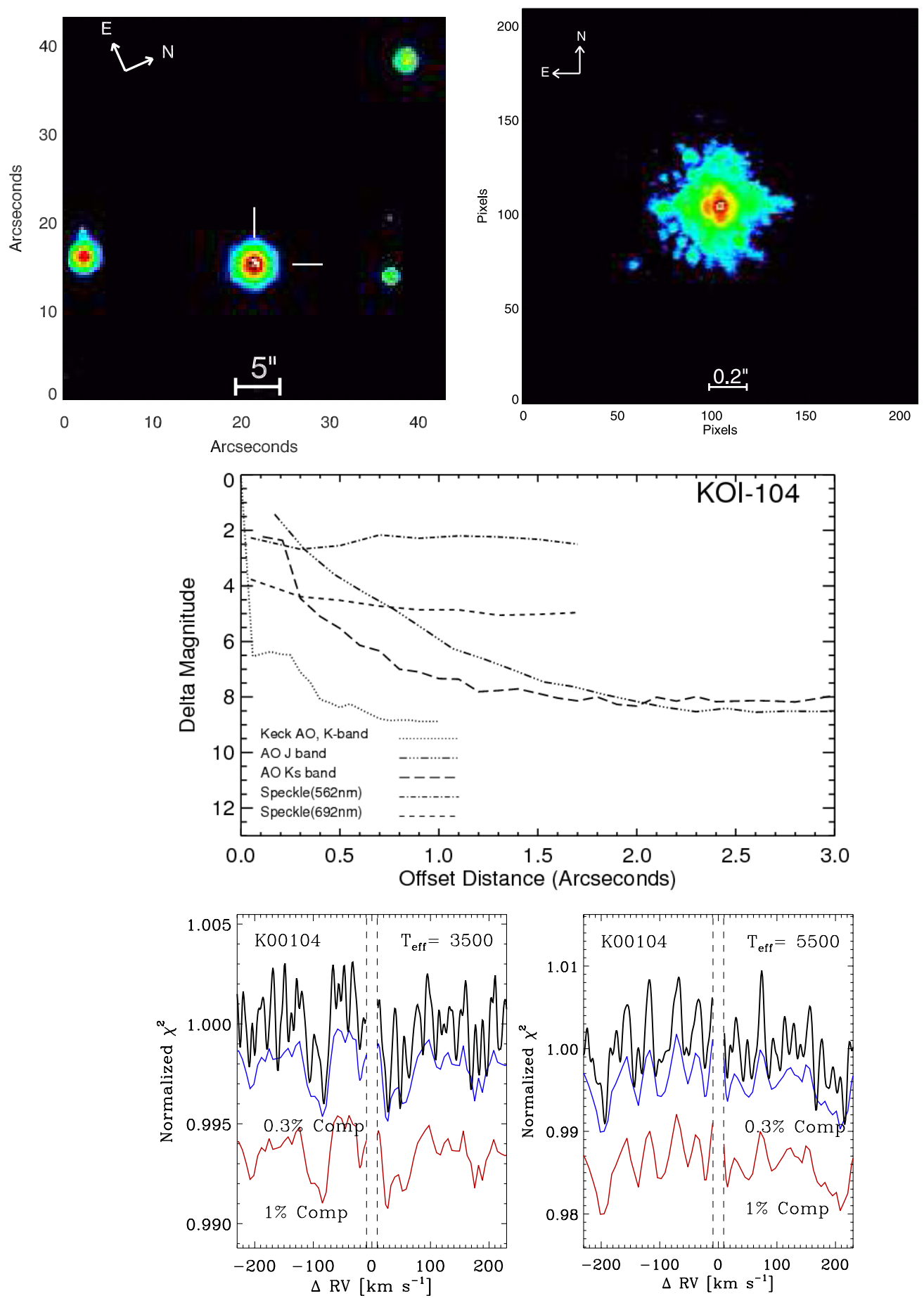

Figure 7. Upper left: seeing-limited image for Kepler-94 (KOI-104). Upper right: adaptive optics image. Middle: limiting magnitudes of companion stars found with Speckle and AO imaging. Lower left and lower right: same as Figure 1, but for Kepler-94. No companions are detected. Any companion brighter than 0.5\% the brightness ( $V$ band) of the primary would have been detected.

(A color version of this figure is available in the online journal.)

of the Kepler transit light curves and Keck RVs. Final stellar parameters are determined by self-consistent fits of the Kepler light curve and RVs to a model of a planet transiting its host star (see below). The output stellar masses and radii differ from input values by typically less than $10 \%$. The Kepler transit light curve shape and orbital period (notably transit duration) implicitly further constrain the stellar density and hence further constrain stellar radius and mass. By solving for all stellar (and planet) parameters simultaneously, and by constraining the fit with priors on $T_{\text {eff }}, \log g$, and metallicity, along with Yonsei-Yale stellar isochrones, we obtain final values of stellar radius and mass, along with planet parameters. Excellent discussions of the iterative convergence of spectroscopic and asteroseismology results, along with self-consistent light curve analysis, are provided by Torres et al. (2012), Gilliland et al. (2013), and Borucki et al. (2013). The final values of all stellar parameters 

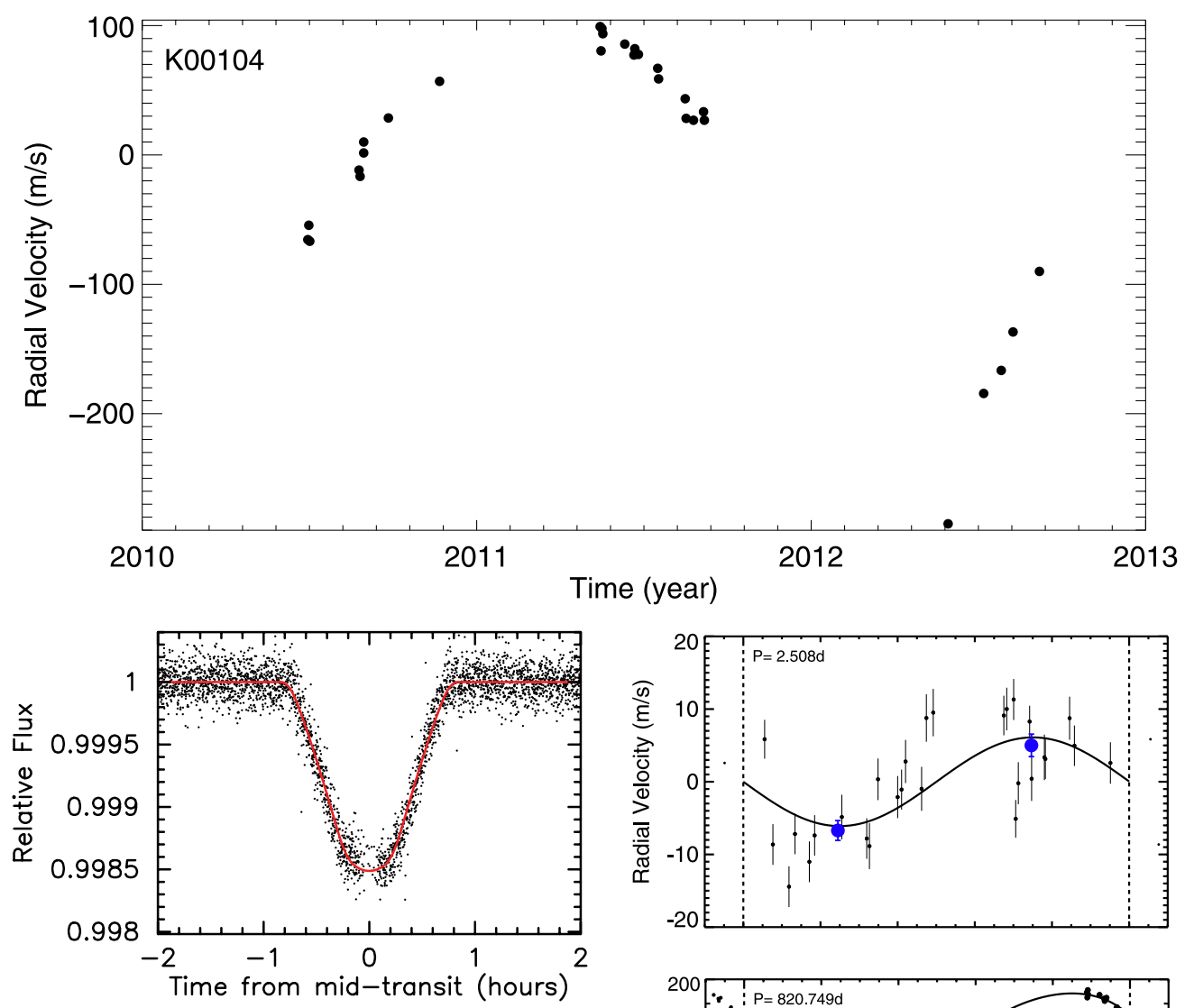

Non-Transiting

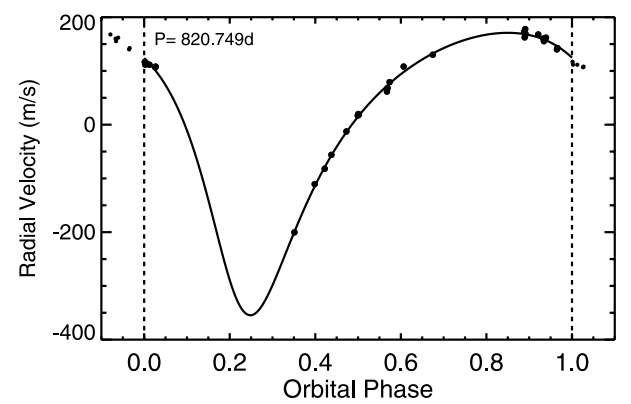

Figure 8. Top: RV vs. time for Kepler-94 (KOI-104). Lower left: phase-folded Kepler photometry for the transiting planet. The non-transiting planet has no light curve. Lower right: phase-folded radial velocities for each planet. The blue points represent binned RVs near quadrature, same as Figure 2 . Kepler-94b: $R p=3.51 \pm 0.15 R_{\oplus}$, $\mathrm{Mp}=10.84 \pm 1.4 M_{\oplus}$. Kepler-94c: $\mathrm{Rp}=\mathrm{NA}, \mathrm{Mp}=3126 \pm 202 M_{\oplus}$, period $=820 \pm 3$ days.

(A color version of this figure is available in the online journal.)

are listed in Table 1. In the following sections, these stellar parameters are used, along with the Kepler photometry, RVs, and stellar structure models, to derive the properties of the 42 planet candidates, listed in Table 2, and the false-positive probabilities (FPPs) listed in Table 3 and discussed in Section 6.

\section{KECK-HIRES PRECISE VELOCITY MEASUREMENTS}

We observed the 22 KOIs with the HIRES spectrometer at the Keck Observatory from 2009 July to 2013 August, obtaining 20-50 RV measurements for each star. The setup used for the RV observations was the same as used by the California Planet Search (CPS), including a slit width of 0 '.87, yielding a resolving power of $R \approx 60,000$ between wavelengths 3600 and $8000 \AA$ (Marcy \& Butler 1992a; Marcy et al. 2008). For those bright $(V<10)$ FGKM stars in the CPS, the photonlimited $\mathrm{RV}$ precision of $\sim 1.5 \mathrm{~m} \mathrm{~s}^{-1}$ matched the typical $\mathrm{RV}$ fluctuations (jitter) from complex gas flows in the photosphere, also $\sim 1.5 \mathrm{~m} \mathrm{~s}^{-1}$, on time scales from minutes to years (Howard et al. 2010).

For the KOIs observed here, typical errors were slightly higher. The typical exposure times were 20-45 minutes (for $K p=10-13 \mathrm{mag}$ ), resulting in an $\mathrm{S} / \mathrm{N}$ between 70 and 200 per pixel, depending on the brightness of the target. As a benchmark, at $K p=13.0 \mathrm{mag}$, the typical exposure was 45 minutes, giving $\mathrm{S} / \mathrm{N}=75$ per pixel, and each pixel spanned $\sim 1.3 \mathrm{~km} \mathrm{~s}^{-1}$. With such exposures, photon statistics of the observed spectrum, along with the comparable $\mathrm{S} / \mathrm{N}$ of the comparison template spectrum, limited the RV precision to $\sim 2 \mathrm{~m} \mathrm{~s}^{-1}$, slightly greater than typical jitter of $\sim 1 \mathrm{~m} \mathrm{~s}^{-1}$ and systematic errors, also of $\sim 1 \mathrm{~m} \mathrm{~s}^{-1}$. Indeed, KOIs yielding non-detections typically have an rms of the RVs of $\sim 3 \mathrm{~m} \mathrm{~s}^{-1}$, as shown in Table 4 . We note that at $\mathrm{S} / \mathrm{N}=70$, uncertainties in wavelength scale are estimated to be less than $0.5 \mathrm{~m} \mathrm{~s}^{-1}$ due to the wavelength information contained in thousands of iodine lines, making wavelength errors a minor source of error compared to the astrophysical jitter of $1.5 \mathrm{~m} \mathrm{~s}^{-1}$. 

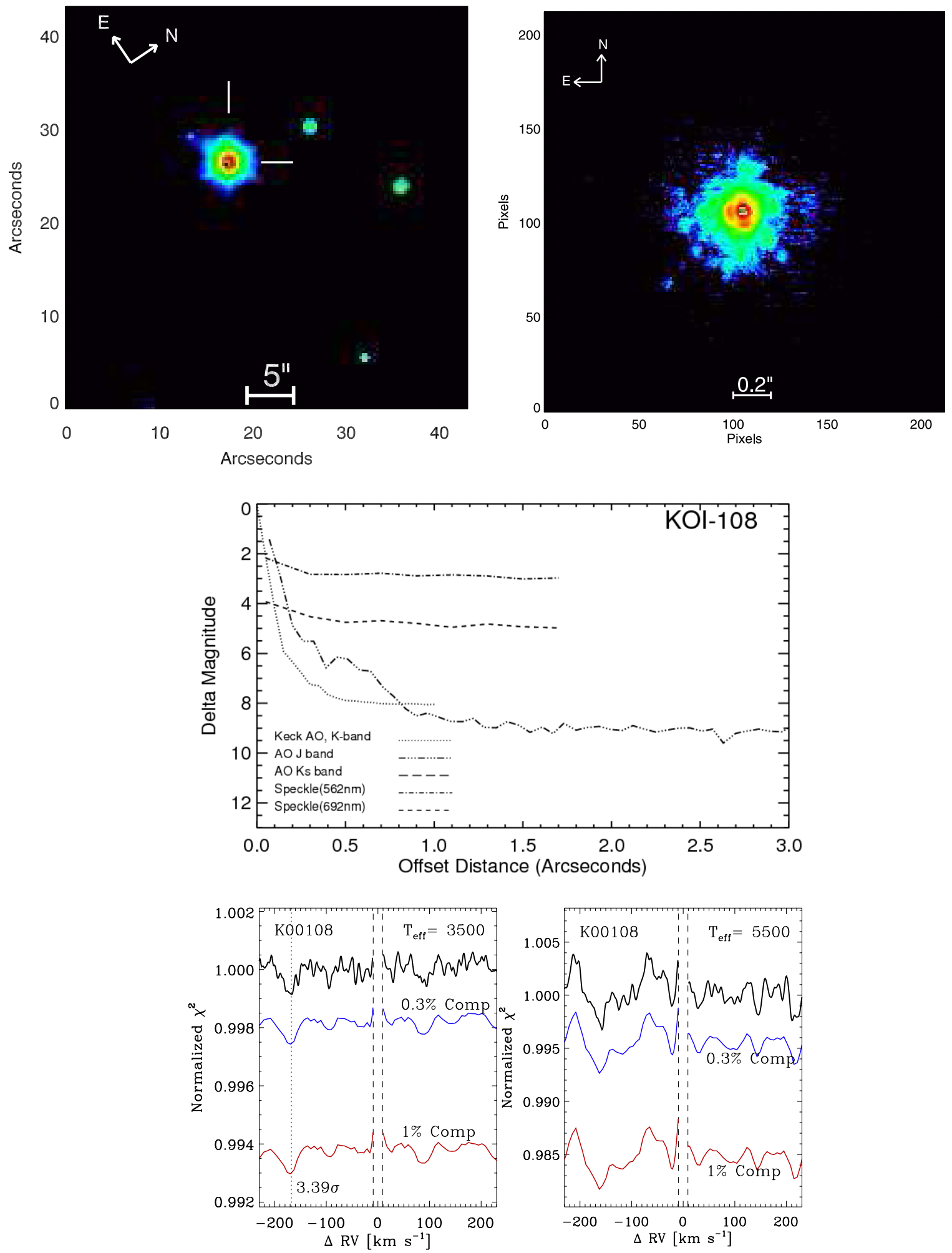

Figure 9. Upper left: seeing-limited image for Kepler-103 (KOI-108). Upper right: adaptive optics image. Middle: limiting magnitudes of companion stars found with Speckle and AO imaging. Lower left and lower right: same as Figure 1, but for Kepler-103. No companions are detected. Any companion brighter than $0.3 \%$ the brightness ( $V$ band) of the primary would have been detected.

(A color version of this figure is available in the online journal.)

The raw reduction of the CCD images followed the standard pipeline of the CPS group, but with the addition of sky subtraction, made necessary by the faint stars and longer exposure times. The spectra were obtained with the iodine absorption cell in front of the entrance slit of the spectrometer, superimposing iodine lines directly on the stellar absorption line spectrum, providing both the observatoryframe wavelength scale and the instrumental profile of the 

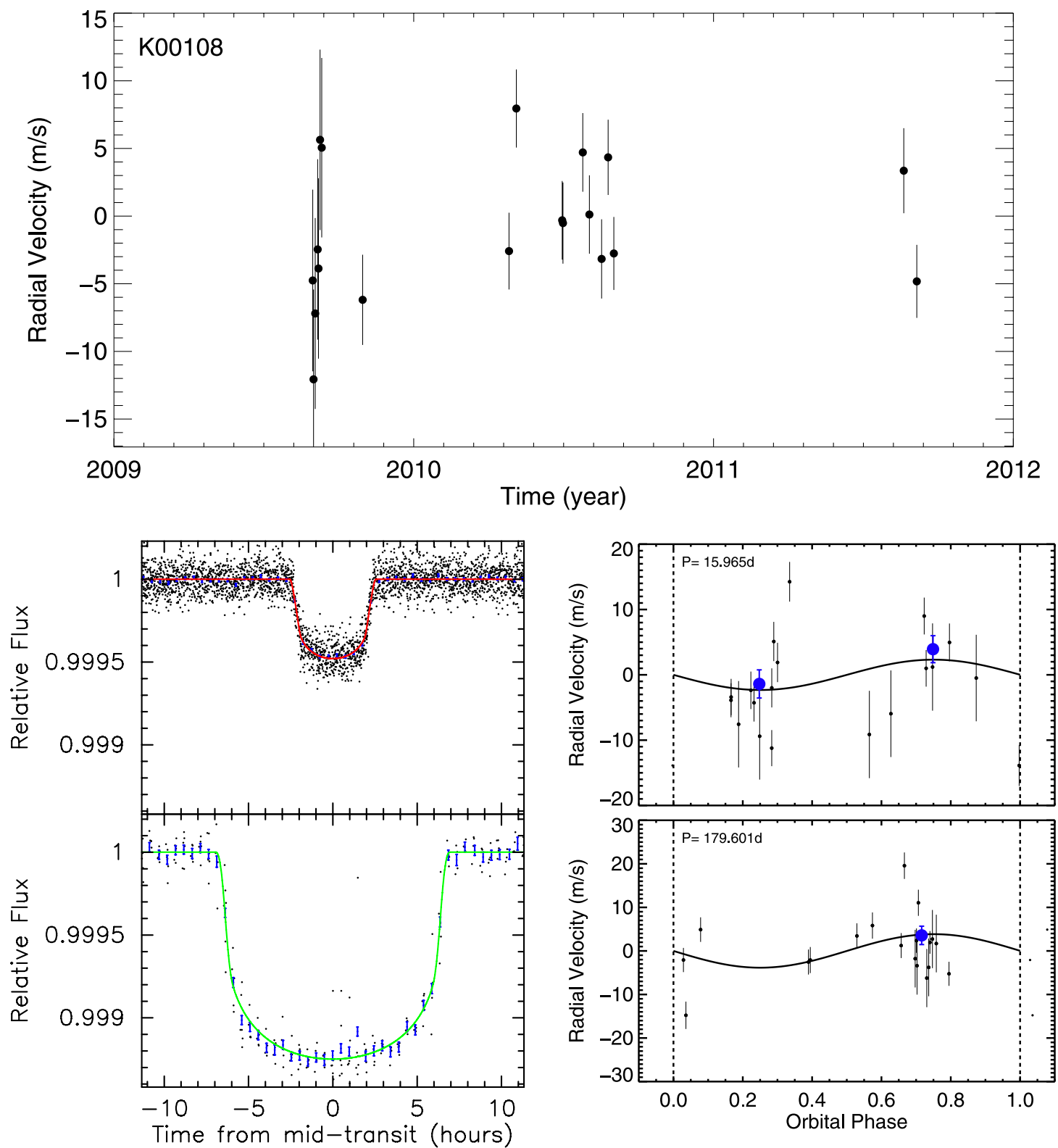

Figure 10. Top: RV vs. time for Kepler-103 (KOI-108). Lower left: phase-folded Kepler photometry for each planet. Lower right: phase-folded radial velocities for each planet. The blue points represent binned RVs near quadrature, same as Figure 2. Kepler-103b: Rp $=3.37 \pm 0.09 R_{\oplus}, \mathrm{Mp}=9.7 \pm 8.6 M_{\oplus}$. Kepler-103c: Rp $=$ $5.14 \pm 0.14 R_{\oplus}, \mathrm{Mp}=36.1 \pm 25.2 M_{\oplus}$.

(A color version of this figure is available in the online journal.)

HIRES spectrometer at each wavelength (Marcy \& Butler 1992b).

The Doppler analysis is the same as that used by the CPS group (Johnson et al. 2010). "Template" spectra obtained without iodine gas in the beam are used in the forward modeling of spectra taken through iodine to solve simultaneously for the wavelength scale, the instrumental profile, and the RV in each of 718 segments of length 80 pixels corresponding to $\sim 2.0 \AA$, depending on position along each spectral order. The internal uncertainty in the final RV measurement for each exposure is the weighted uncertainty in the mean RV of those 718 segments, the weights of which are determined dynamically by the RV scatter of each segment relative to the mean RV of the other segments. The resulting weights reflect the actual RV performance quality of each spectrum segment. The template spectra are also used in spectroscopic analysis to determine stellar parameters, as described in Section 3.

The typical long exposures of 10-45 minutes and modest $\mathrm{S} / \mathrm{N}$ of the stellar spectra imply that night sky emission lines and scattered moonlight may significantly contaminate the spectra. To measure and remove the contaminating light we use the $\mathrm{C} 2$ decker on HIRES which projects to $0.87 \times 14^{\prime \prime} .0$ on the sky. The C2 decker collects both the stellar light and night-sky light simultaneously. The star is guided at the center of the slit while the sky light passes through the entire $14^{\prime \prime}$ length of the slit. The sky contamination is thus simultaneously recorded with the stellar spectrum at each wavelength in the regions above and below each spectral order, beyond the wings of the PSF of the star image projected onto the CCD detector. The "sky pixels" located above and below each spectral order provide 

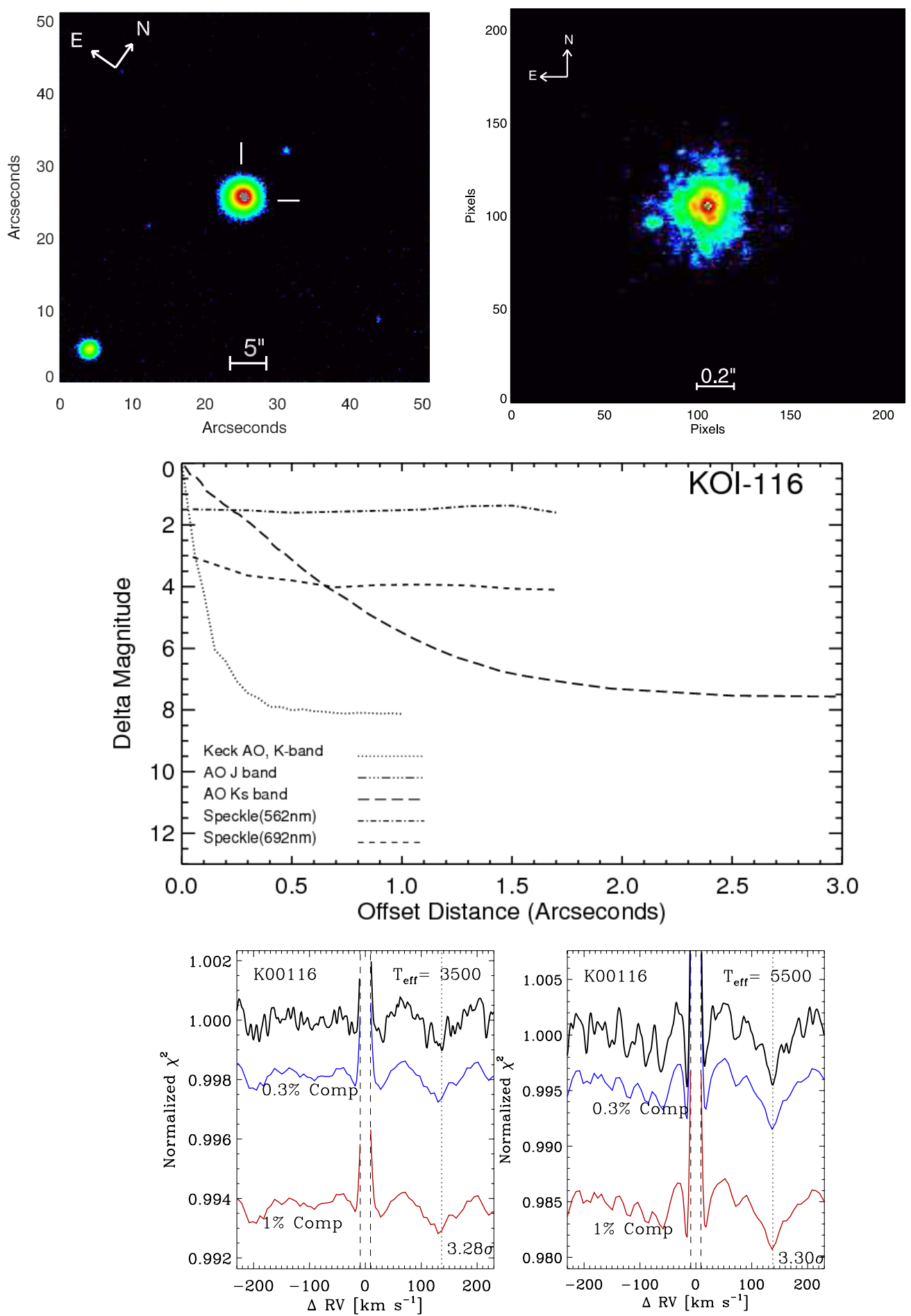

Figure 11. Upper left: seeing-limited image for Kepler-106 (KOI-116). Upper right: adaptive optics image. Middle: limiting magnitudes of companion stars found with Speckle and AO imaging. Lower left and lower right: same as Figure 1, but for Kepler-106. No companions are detected. Any companion brighter than 0.3\% the brightness ( $V$ band) of the primary would have been detected.

(A color version of this figure is available in the online journal.)

a direct measure of the spectrum of the sky and we subtract that sky light on a column by column basis (wavelength by wavelength). When the seeing is greater than 1".5 (which occurs less than $10 \%$ of the time at Mauna Kea), we do not use the $\mathrm{C} 2$ decker but instead use a smaller slit of dimensions $0.87 \times 3.5$ (B5 decker) and we observe only bright stars, $K p<11 \mathrm{mag}$, with exposure times of $\sim 10$ minutes to avoid sky contamination.
Observations of KOIs acquired in 2009 did not employ the $\mathrm{C} 2$ decker. With no ability to perform sky subtraction, those observations have additional RV errors from scattered moonlight. We quantified these RV errors by studying the contamination seen in long-slit spectra and by comparing the scatter in the RVs during 2009 (no sky subtraction) to the RVs obtained in later years (with sky subtraction), permitting us to compute the additional RV uncertainties incurred in 2009. 

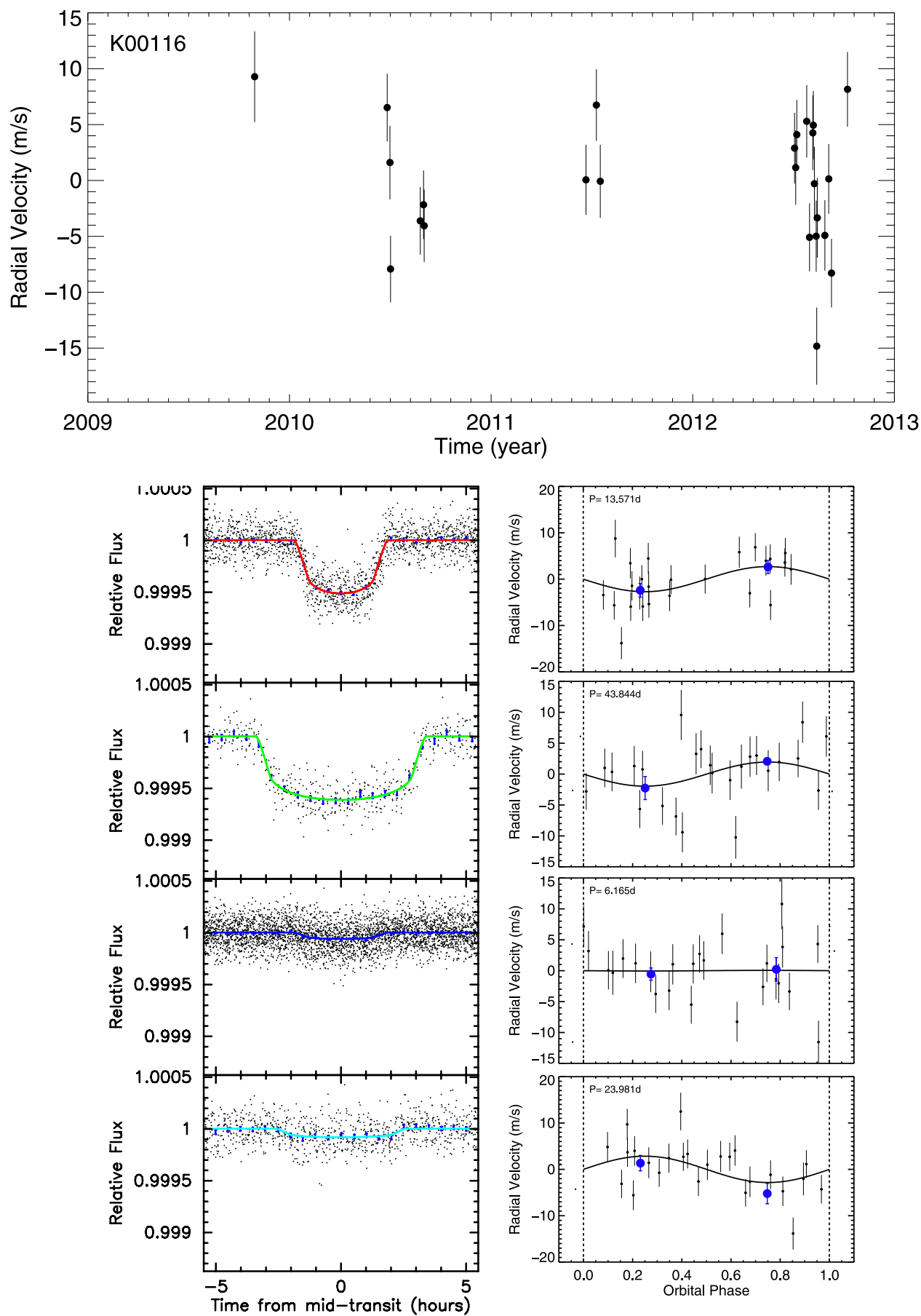

Figure 12. Top: RV vs. time for Kepler-106 (KOI-116). Lower left: phase-folded Kepler photometry for each planet. Lower right: phase-folded radial velocities for each planet. The blue points represent binned RVs near quadrature, same as Figure 2. Kepler-106c: Rp $=2.50 \pm 0.32 R_{\oplus}, \mathrm{Mp}=10.44 \pm 3.2 M_{\oplus}$. Kepler-106e: Rp $=$ $2.56 \pm 0.33 R_{\oplus}, \mathrm{Mp}=11.17 \pm 5.8 M_{\oplus}$. Kepler-106b: Rp $=0.82 \pm 0.11 R_{\oplus}, \mathrm{Mp}=0.15 \pm 2.8 M_{\oplus}$. Kepler-106d: $\mathrm{Rp}=0.95 \pm 0.13 R_{\oplus}, \mathrm{Mp}=-6.39 \pm 7.0 M_{\oplus}$

(A color version of this figure is available in the online journal.)

In typical gibbous moon conditions with light clouds, the moonlight contributed $1 \%-2 \%$ of the light of a $K p=13 \mathrm{mag}$ star (Rayleigh scattering causing a wavelength dependence) within a projected $\sim 3^{\prime \prime} .5$ extraction width of each spectral order. Under such gibbous conditions, the moonlit sky at Mauna Kea is apparently 19th mag per square arcsec in $V$ band. Increasing amounts of cirrus clouds will scatter more moonlight into the slit but will transmit less star light, thereby increasing the relative amount of contamination of the stellar spectrum.

We find that RV errors of up to $10 \mathrm{~m} \mathrm{~s}^{-1}$ occurred during 2009, depending on the amount of contamination and the relative RV of the stellar spectrum and the scattered solar spectrum from the moon. Employing sky subtraction with the C2 decker yield RV precision as if no sky contamination occurred; the observed RV scatter does not depend on the phase or presence of the moon. For stars brighter than $K p=11 \mathrm{mag}$ the sky subtraction made no difference in RV precision as moon light was apparently negligible.

Plots of the RVs for each of the 42 transiting planet candidates, phased to the final orbit (see Section 5), are shown in Figures 2-44. The measured RVs for each of the 22 KOIs are listed in Table 4. In those tables, the first column contains 

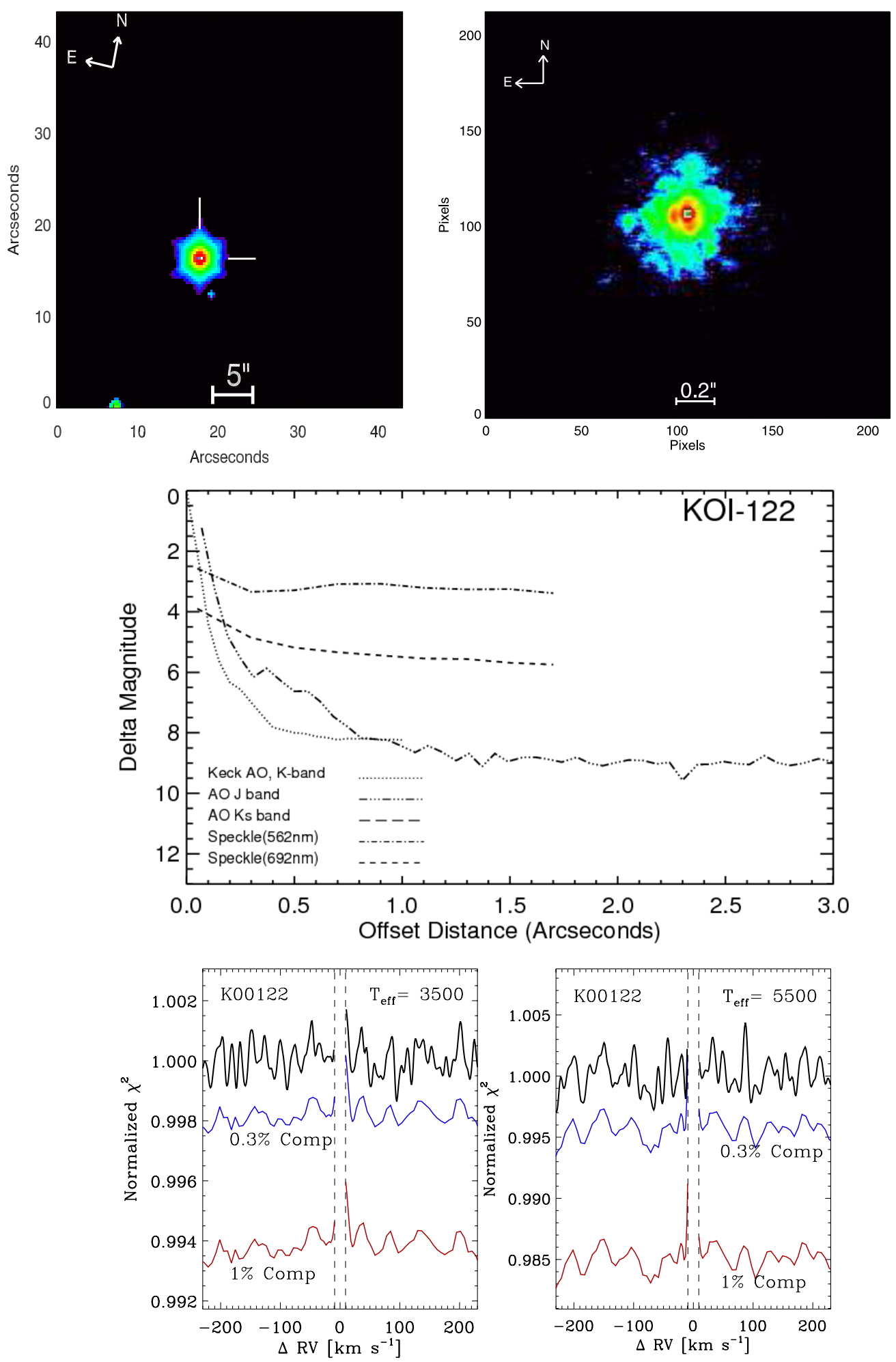

Figure 13. Upper left: seeing-limited image for Kepler 95 (KOI-122). Upper right: adaptive optics image. Middle: limiting magnitudes of companion stars found with Speckle and AO imaging. Lower left and lower right: same as Figure 1, but for Kepler 95. No companions are detected. Any companion brighter than $0.3 \%$ the brightness ( $V$ band) of the primary would have been detected.

(A color version of this figure is available in the online journal.)

the barycentric Julian date when the star light arrived at the solar system barycenter (BJD) based on the measured photonweighted mid-time of the exposure. The second column contains the relative RV (with no defined RV zero point) in the frame of the barycenter of the solar system. Only the changes with time in the RVs are physically meaningful for a given star, not the individual RV values. The absolute RVs can be determined relative to the solar system barycenter, but only with an accuracy of $\sim 50 \mathrm{~m} \mathrm{~s}^{-1}$ (Chubak et al. 2012). The third column contains the time-series RV uncertainty, which includes both the internal 

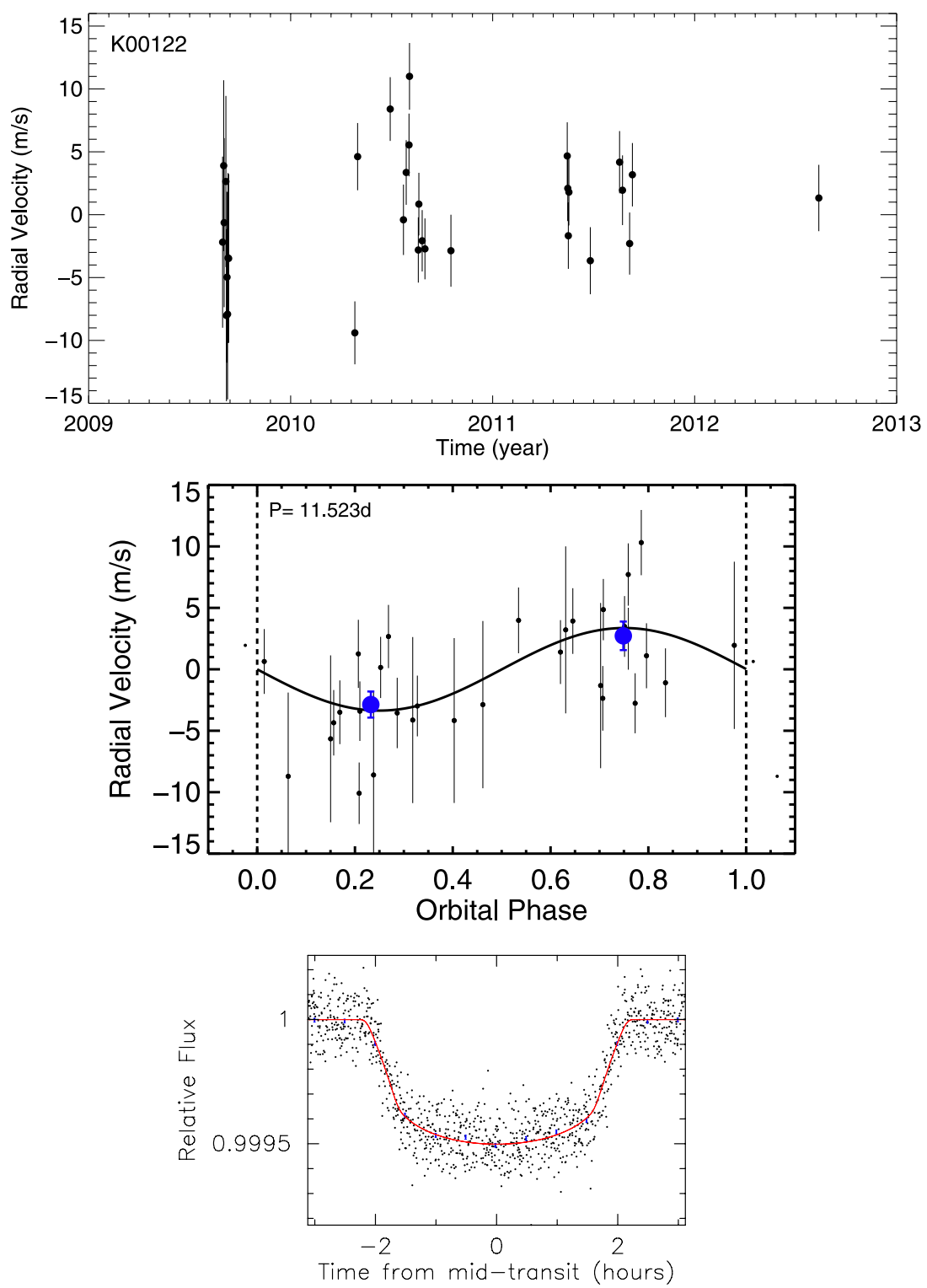

Figure 14. Top: RV vs. time for KOI-122. Lower left: phase-folded Kepler photometry for each planet. Lower right: phase-folded radial velocities for each planet. The blue points represent binned RVs near quadrature, same as Figure 2. Kepler 95b: Rp $=3.42 \pm 0.09 R_{\oplus}, \mathrm{Mp}=13.0 \pm 2.9 M_{\oplus}$.

(A color version of this figure is available in the online journal.)

uncertainty (from the uncertainty in the mean Doppler shift of 718 spectral segments) and an approximate jitter of $2 \mathrm{~m} \mathrm{~s}^{-1}$ (from photospheric and instrumental sources) based on hundreds of stars of similar FGK spectral type (Isaacson \& Fischer 2010).

The actual RV jitter has values between $1-3 \mathrm{~m} \mathrm{~s}^{-1}$ for individual stars, but the actual photospheric fluid flows for any particular star and the detailed systematic RV errors are both difficult to estimate with any accuracy better than $1 \mathrm{~m} \mathrm{~s}^{-1}$. The jitter is added in quadrature to the internal uncertainty for each RV measurement, to yield a final RV uncertainty. The actual uncertainties are surely non-Gaussian from both the photospheric hydrodynamics and from systematic errors in the Doppler analysis, and they are likely to be temporally coherent with separate power spectra. Such error distributions are difficult to characterize precisely. Still, it is marvelous that the Doppler-shift errors for 13th magnitude stars located hundreds of light years away are less than human jogging speed.

\section{PLANET CHARACTERIZATION}

We determine the physical and orbital properties of the 42 transiting planet candidates around the 22 KOIs by simultaneously fitting Kepler photometry and Keck RVs with an analytical model of a transiting planet (Mandel \& Agol 2002). To build these models, we started with an adopted stellar density as determined by either the SME analysis of the high-resolution Keck spectrum of the star or the accompanying asteroseismology analysis (both described in Section 3). The models assume Keplerian orbits with no gravitational interactions between the planets of the multiple-planet systems. This non-interaction assumption is adequate to yield parameters as accurate as the limited time series permits, as any precession or secular resonances will create detectable effects (by RVs) only after a decade, even for periods as short as weeks. The parameters in the model include the stellar density (initially from the SME or asteroseismology analysis), the RV gamma (center of mass velocity), a mean 

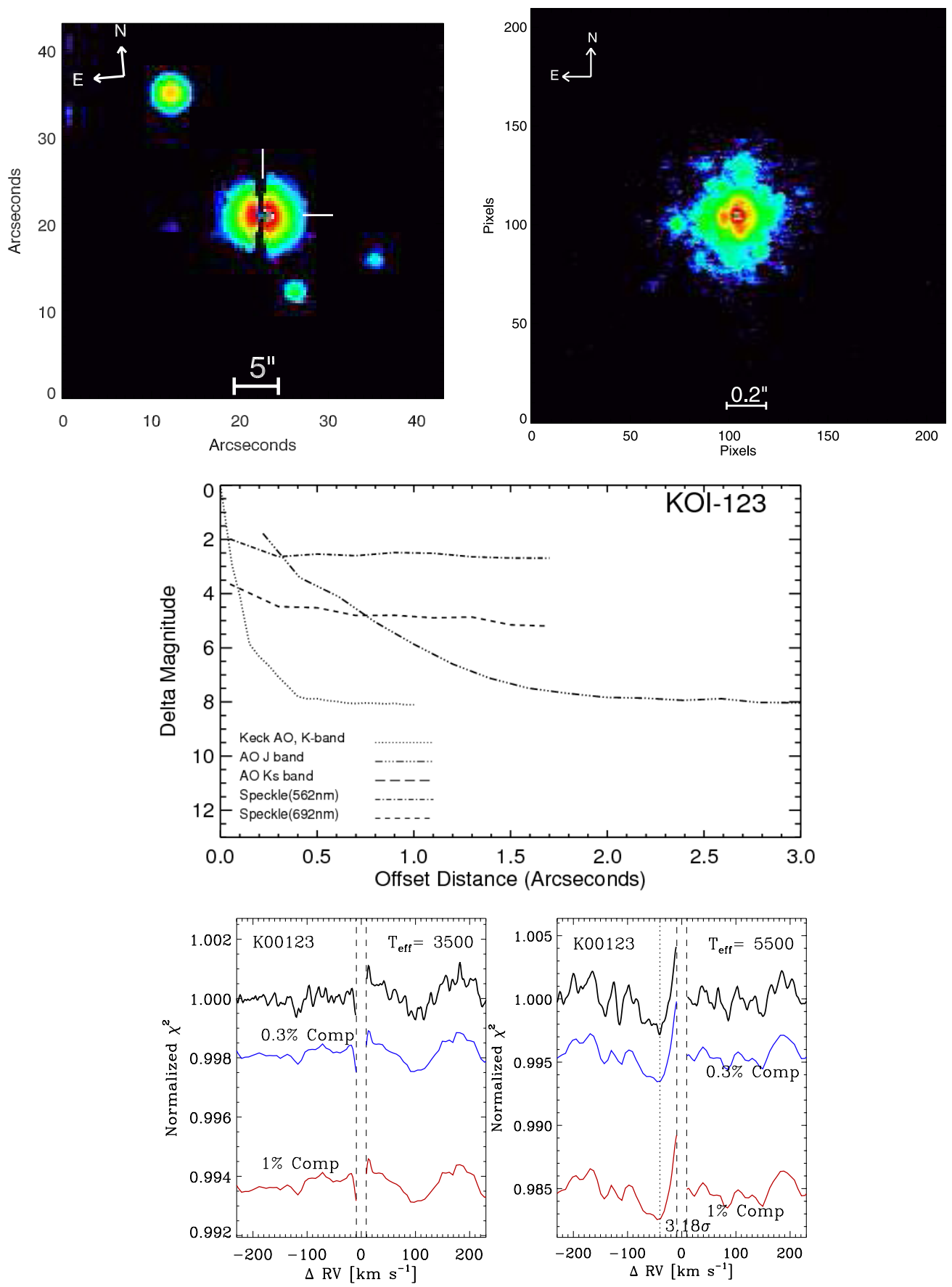

Figure 15. Upper left: seeing-limited image for Kepler-109 (KOI-123). Upper right: adaptive optics image. Middle: limiting magnitudes of companion stars found with Speckle and AO imaging. Lower left and lower right: same as Figure 1, but for Kepler-109. No companions are detected. Any companion brighter than $0.3 \%$ the brightness ( $V$ band) of the primary would have been detected.

(A color version of this figure is available in the online journal.)

photometric flux, an RV zero point, the time of one transit $(T 0)$, orbital period $(P)$, impact parameter $(b)$, the scaled planet radius $\left(R_{\mathrm{PL}} / R_{*}\right)$, and the RV amplitude $(K)$.

We use the parameterization of limb-darkening (Mandel \& Agol 2002) with coefficients calculated by Claret \& Bloemen (2011) for the Kepler bandpass. We simultaneously fit all measurements with a model using an MCMC routine. To determine planet mass and radius the Markov chains from the stellar modeling are combined with the Markov chains from the transit model. For each Markov chain in the transit model, we pick a stellar model from the stellar evolution model Markov chain and calculate planet radius and mass. This produces a posterior distribution for radius and mass from which we measure the median and uncertainties.

The final values of the planet parameters in Table 2 are the values at which the posterior distribution is a maximum, often termed the "mode" of the distribution.

We considered both eccentric and circular orbits for the models of all transiting planets. A comparison of the chisquare statistic from the best-fitting models for circular and eccentric orbits showed that in no cases was a non-zero eccentricity demanded, or even compelling. The best-fitting RV 

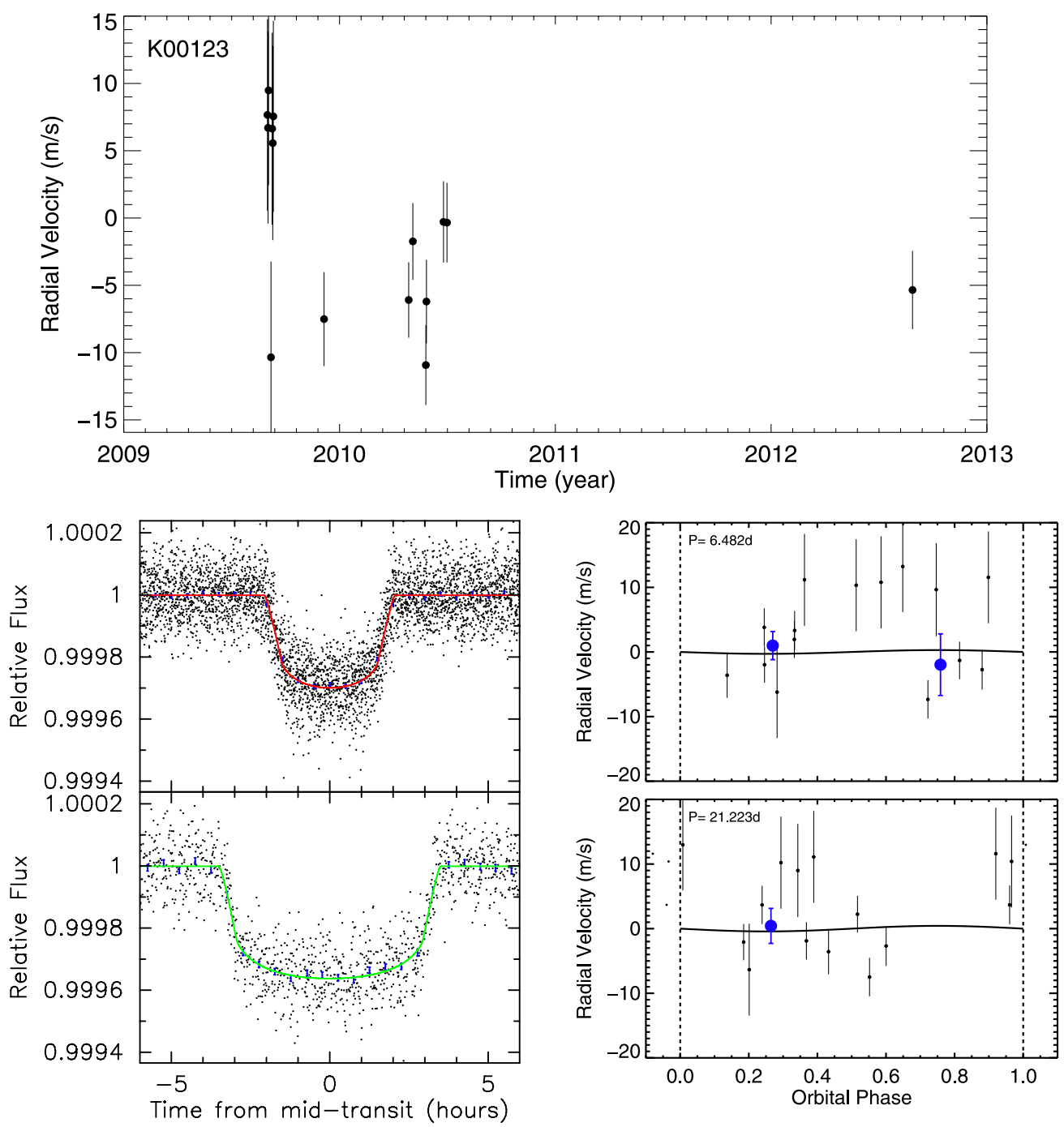

Figure 16. Top: RV vs. time for Kepler-109 (KOI-123). Lower left: phase-folded Kepler photometry for each planet. Lower right: phase-folded radial velocities for each planet. The blue points represent binned RVs near quadrature, same as Figure 2. Kepler-109b: Rp $=2.37 \pm 0.07 R_{\oplus}, \mathrm{Mp}=1.3 \pm 5.4 M_{\oplus}$. Kepler-109c: Rp $=$ $2.52 \pm 0.07 R_{\oplus}, \mathrm{Mp}=2.22 \pm 7.8 M_{\oplus}$.

(A color version of this figure is available in the online journal.)

semi-amplitude, $K$, for all transiting planets in this study is less than $6.1 \mathrm{~m} \mathrm{~s}^{-1}$ (for Kepler-94b), only a factor of two or three larger than the RV errors. This modest $\mathrm{S} / \mathrm{N}$ for the RVs limits their capability to detect eccentricities securely. We found that models with non-zero eccentricities open the door for peculiar and undefended Keplerian orbits that predict high acceleration during periastron passages where no RVs were obtained. These models predict wild, brief departures (during periastron) of the RVs from the measured standard deviation and thus violate Occam's Razor that favors the simplest possible model that satisfies the RV data. Therefore, all models of the transiting planets were computed with a circular orbit. Only the RVs for Kepler-94b exhibit some evidence of an eccentricity near $e=0.2$, but the non-zero eccentricity is not compelling (see Section 7.4).

For the 7 non-transiting planets, non-zero eccentricities are commonly demanded by the non-sinusoidal and large (many sigma) RV variations. The RVs for three non-transiting planets revealed evidence of non-zero eccentricities, namely for KOIs Kepler-94c, Kepler-25d, Kepler-68d. Table 2 lists the best-fitting orbital parameters for those four planets. The derived eccentric- ities of $0.38 \pm 0.05,0.18 \pm 0.10$, and $0.10 \pm 0.04$ respectively. The best-fit values of $\omega$ are $157^{\circ} \pm 6^{\circ}, 51^{\circ} \pm 70^{\circ}, 347^{\circ} \pm 100^{\circ}$, respectively.

In all models, we allowed the value of the RV amplitude to be negative as well as positive, corresponding to both negative and positive values of planet mass. Obviously negative mass is not physically allowed. But fluctuations in the RV measurements due to errors may result in RVs that are anti-correlated with the ephemeris of the planet as dictated by the photometric light curve. Fluctuations can spuriously cause the RVs to be slightly positive when orbital phase dictates they should be negative, and vice versa. In such cases, the derived negative mass, and the posterior distribution of masses, is a statistically important measure of the possible masses of the planet, especially useful when included with the ensemble of masses of other planets and their posterior mass distributions. By allowing planet masses to float negative, we account for the natural fluctuations in planet mass from RV errors.

For all planet candidates, especially those that yielded less than $2 \sigma$ detections of the RV signal ( $K$ less than $2 \sigma$ from zero), we also compute the 95th percentile upper limit to the 

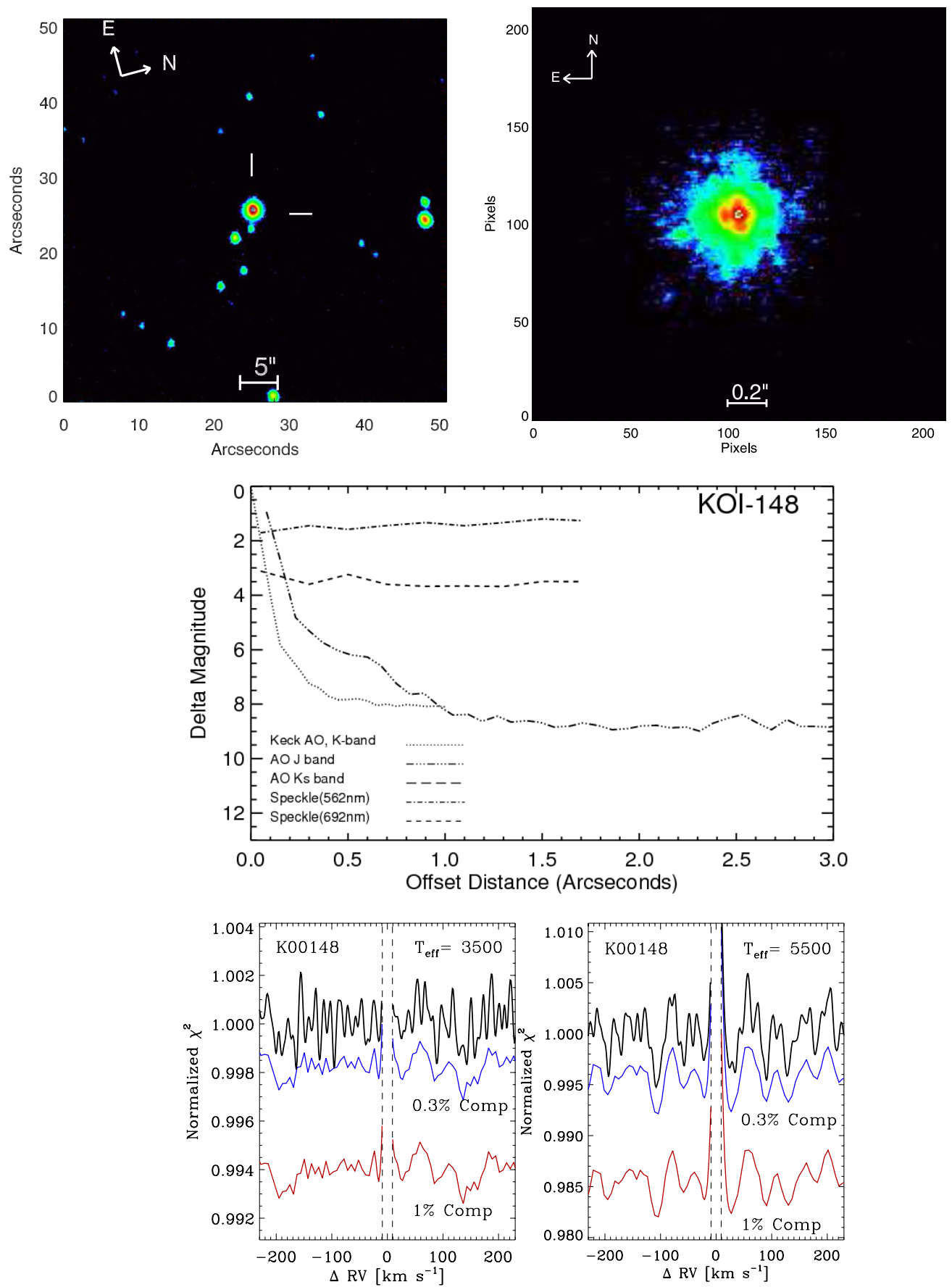

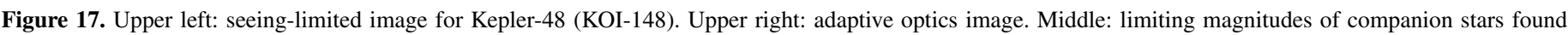

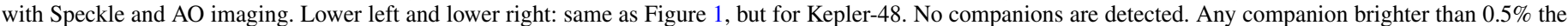
brightness ( $V$ band) of the primary would have been detected.

(A color version of this figure is available in the online journal.)

planet mass. To compute this for each planet, we integrated the posterior mass distribution from the MCMC analysis to determine the mass at the 95 th percentile. This 95 th percentile serves as a useful metric of an "upper limit" to the planet mass, and there remains a 5\% probability that the actual planet mass is higher. In many cases, the posterior mass distribution formally permits the planet's mass to be zero, or even negative. In such cases, the physically acceptable upper limit, such as that computed from the 95th percentile, offers a useful upper bound on the actual mass of the planet. For such planets, we determine both metrics of planet mass for these non-detections.
The planet mass at the peak of the posterior distribution can be positive or negative, which is useful for statistical treatment of the planets as an ensemble. The 95th percentile upper limit is positive, useful for constraining planet mass, density, and chemical composition. In Table 2, Column 4 gives the planet mass at the peak of the posterior distribution and Column 5 give the 95th percentile upper limit.

For each KOI, we plot the RVs as a function of time, the phase-folded RVs for each transiting and non-transiting planet, and the phase-folded Kepler photometry. (Figures 1-43). The errors for each RV measurement include the internal error and 

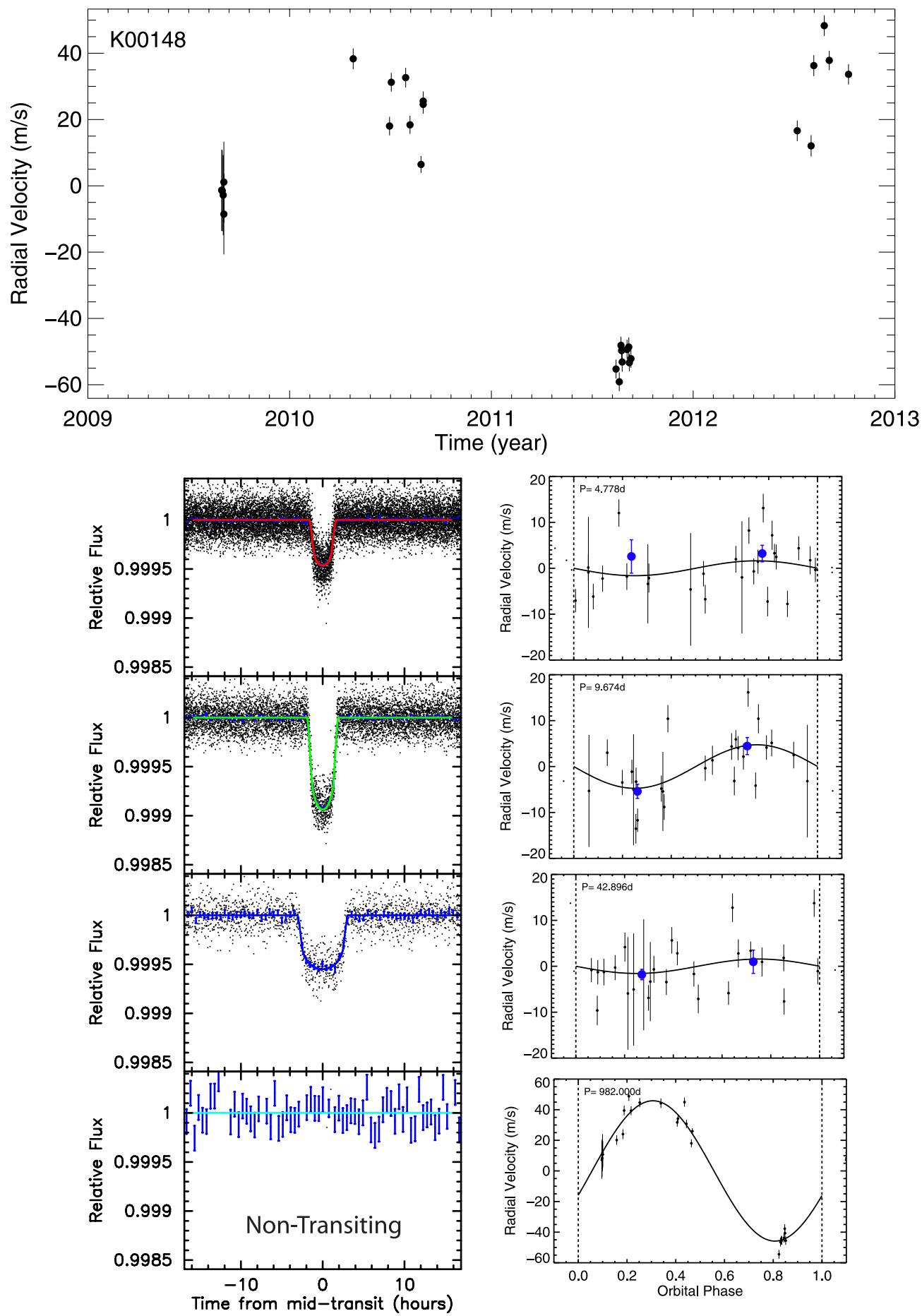

Figure 18. Top: RV vs. time for Kepler-48 (KOI-148). Lower left: phase-folded Kepler photometry for each planet. Lower right: phase-folded radial velocities for each planet. The blue points represent binned RVs near quadrature, same as Figure 2. Kepler-48b: Rp $=1.88 \pm 0.10 R_{\oplus}, \mathrm{Mp}=3.94 \pm 2.1 M_{\oplus}$. Kepler-48c: Rp $=$ $2.71 \pm 0.14 R_{\oplus}, \mathrm{Mp}=14.61 \pm 2.3 M_{\oplus}$. Kepler-48d: $\mathrm{Rp}=2.04 \pm 0.11 R_{\oplus}, \mathrm{Mp}=7.93 \pm 4.6 M_{\oplus}$. Kepler-48e: Rp $=\mathrm{NA}, \mathrm{Mp}=657 \pm 25 M_{\oplus}$, period $=982 \pm 8$ days. (A color version of this figure is available in the online journal.)

$2.0 \mathrm{~m} \mathrm{~s}^{-1}$ of jitter, which is added in quadrature to obtain the final error. In each phase-folded RV plot, the best-fit RV curve is over plotted on top of the RVs. Each blue point is the average of the RVs that fall within one of the two quadrature ranges, $0.25 \pm 0.125$ and $0.75 \pm 0.125$, of phase set from the transits for which RV excursions are expected to be maximum. The value of the binned point consists of the weighted average of the RVs within the bin. The times of observation are also weighted, causing the blue RV point to be slightly offset from 0.25 or
0.75 , based on the average phase of the RVs in each bin. The error of the binned RV is the standard deviation of the RVs within the bin divided by the square root of the number of binned RVs.

In summary, we fit the photometry and RVs with a Mandel \& Agol 2002 model by adopting the star's properties based on spectroscopy (SME) and on asteroseismology, if available. Model parameters are determined by the chi-squared statistic, and we compute posterior distributions for the properties of the 

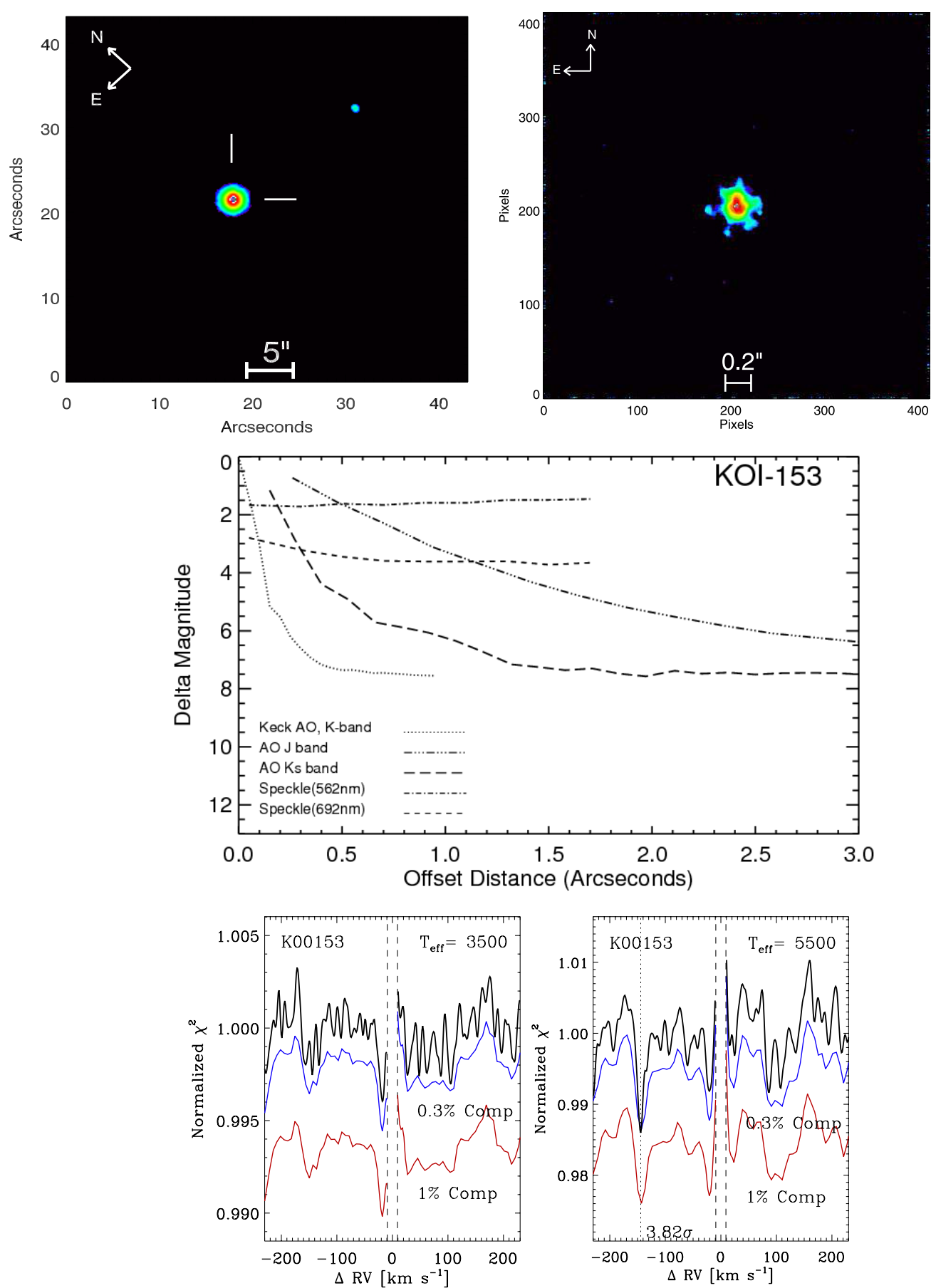

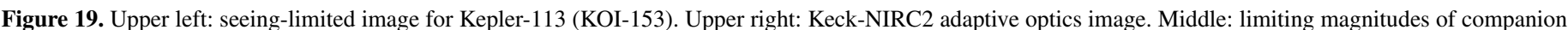

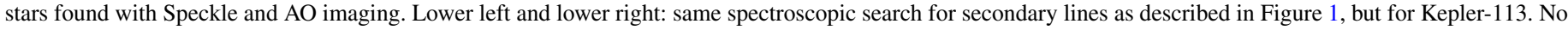
companions are detected. Any companion brighter than $0.5 \%$ the brightness ( $V$ band) of the primary would have been detected.

(A color version of this figure is available in the online journal.)

planet and the star using MCMC. We derive planet radius, mass, orbital period, ephemeris, and stellar parameters, including the mean stellar density, in the final solution. The final stellar parameters for each star are in Table 1. The final planetary parameters are listed in Table 2, including stellar density from the model and unbiased planet masses and densities that can be negative. The associated $1 \sigma$ uncertainty for each parameter is computed by integrating the posterior distribution of a parameter to $34 \%$ of its area on either side of the peak, with values listed in Table 2.

\section{FALSE-POSITIVE ASSESSMENTS}

As has been well documented (Torres et al. 2011), a series of periodic photometric dimmings consistent with a transiting planet may actually be the result of various astrophysical phenomena that involve no planet at all. Such "false-positive" scenarios involve the light from some angularly nearby star located within the $\left(\sim 15^{\prime \prime}\right.$ diameter $)$ Kepler software aperture that dims with a duration and periodicity consistent with an orbiting object passing in front of the target star. The light from 

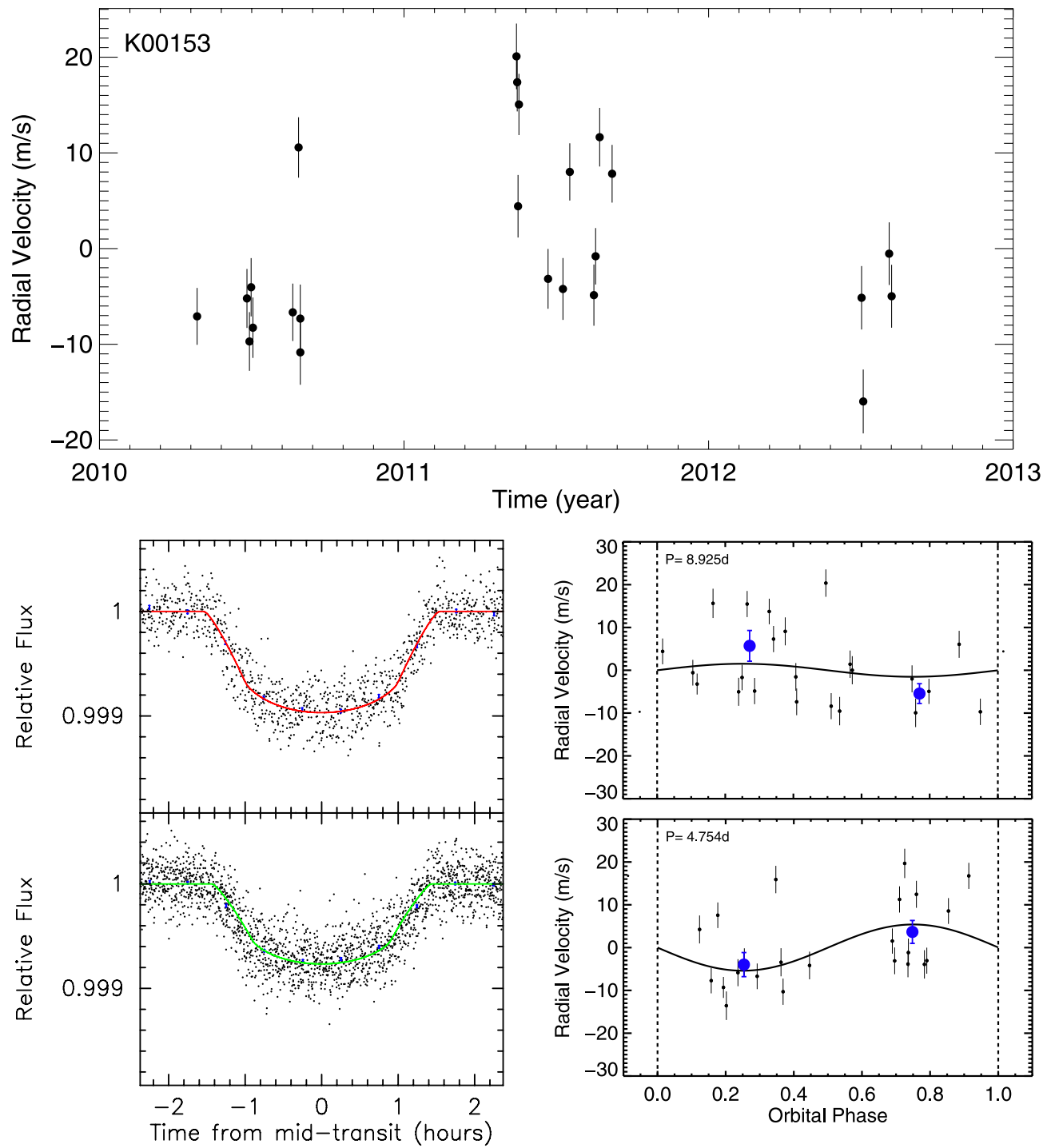

Figure 20. Top: RV vs. time for Kepler-113 (KOI-153). Lower left: phase-folded Kepler photometry for each planet. Lower right: phase-folded radial velocities for each planet. The blue points represent binned RVs near quadrature, same as Figure 2. Kepler-113c: Rp $=2.18 \pm 0.06 R_{\oplus}, \mathrm{Mp}=-4.04 \pm 6.4 M_{\oplus}$. Kepler-113b: Rp $=$ $1.82 \pm 0.05 R_{\oplus}, \mathrm{Mp}=11.7 \pm 4.2 M_{\oplus}$.

(A color version of this figure is available in the online journal.)

that nearby star may be located within the software aperture of the target star or located just outside that aperture so that the wings of its PSF encroach into the aperture, polluting the brightness measurements. The amount of pollution may vary with the quarterly roll of the spacecraft, as each star experiences small changes in both the relative position of its aperture and in its differential aberration from the changing velocity vector of the spacecraft. The polluting nearby star may be physically unrelated to the target star (in the background or foreground) or it may be gravitationally bound, and the cause of its dimming could be a transiting planet, brown dwarf, star, cloud, or other construct.

By considering all astrophysical false-positive scenarios in the direction of the Kepler field of view, and in the absence of follow-up measurements, the FPP for Kepler planet candidates smaller than Jupiter is $\sim 10 \%$ (Morton \& Johnson 2011; Morton 2012; Fressin et al. 2013). However, the 22 KOIs here were selected after follow-up observations had already been done, notably spectroscopy, high-resolution imaging, and careful astrometry, removing many of the apparent false positives, as described in Section 2.3. We thus expect a false-positive rate for the 42 planet candidates studied here to be well below $10 \%$.

For Jupiter-size planet candidates the false-positive rate is higher, near $\sim 35 \%$ (Santerne et al. 2012; Fressin et al. 2013) because both brown dwarfs and $\mathrm{M}$ dwarfs are roughly the size of Jupiter, allowing them to masquerade as giant planets. Also, gas giant planets are geometrically more likely to transit with only some fraction of the planet's apparent disk covering the star's disk. Such "grazing incidence transits" with impact parameter, $b>0.9$, cause " $V$-shaped" light curves that resemble those caused by eclipsing binaries (for the same reason). Thus, the $V$-shaped light curves from gas giants forces the Kepler TCERT planet validation effort to retain both the true planets and the background eclipsing binaries, thereby increasing the occurrence of false positives. However, none of the transiting planets in this work are nearly as large as Jupiter.

The detailed assessment of the FPP for any individual planet candidate requires careful analysis. This "planet-validation" 

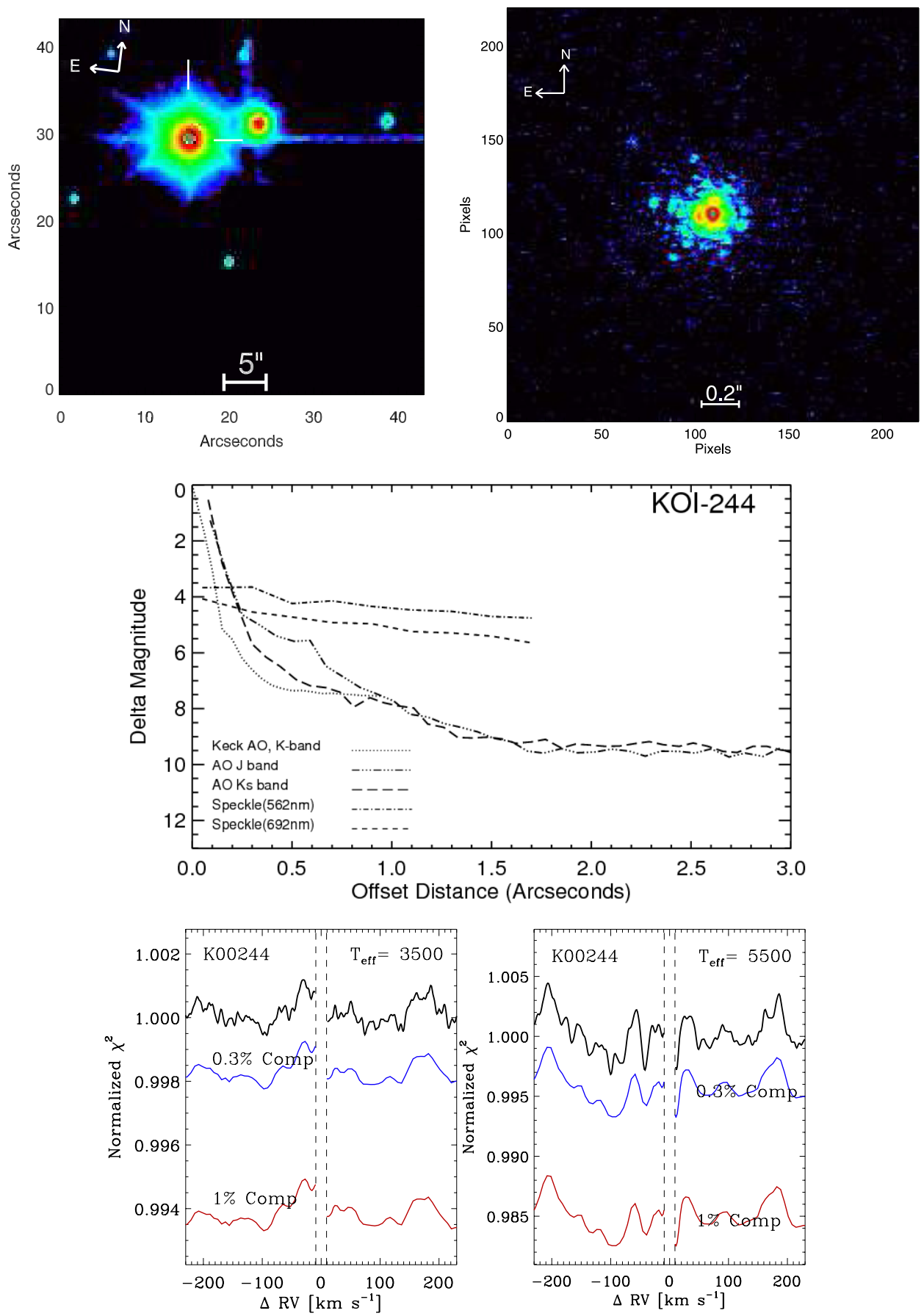

Figure 21. Upper left: seeing-limited image for Kepler-25 (KOI-244). Upper right: adaptive optics image. Middle: limiting magnitudes of companion stars found with Speckle and AO imaging. Lower left and lower right: same as Figure 1, but for Kepler-25. No companions are detected. Any companion brighter than $0.3 \%$ the brightness ( $V$ band) of the primary would have been detected.

(A color version of this figure is available in the online journal.)

process can be aided by the corroborating detection of the planet with some other technique such as with RVs or transittiming measurements. Validation may also be accomplished by estimating the probability that the planet is real (from measured occurrence rates) and comparing it to the sum of the probabilities of all false-positive scenarios that are consistent with the observations.

\subsection{Follow-up Observations Constrain False Positives}

To tighten the estimates of the FPPs for the 42 transiting planet candidates in this paper, we performed a wide variety of follow-up observations, described in Section 2 and its subsections. The follow-up observations include AO imaging, speckle interferometry, and high-resolution spectroscopy, all capable of detecting angularly nearby stars that might be the source of the dimming that mimics a transiting planet around the target star. The $\mathrm{AO}$ and speckle techniques detect companions beyond a few tenths of an arcsec (detailed below) while the spectroscopy detects nearby stars (from secondary absorption lines or asymmetries in spectral lines) located within a few tenths of an arcsec for relative RVs $>10 \mathrm{~km} \mathrm{~s}^{-1}$. Thus these techniques are useful to detect stellar companions located within 

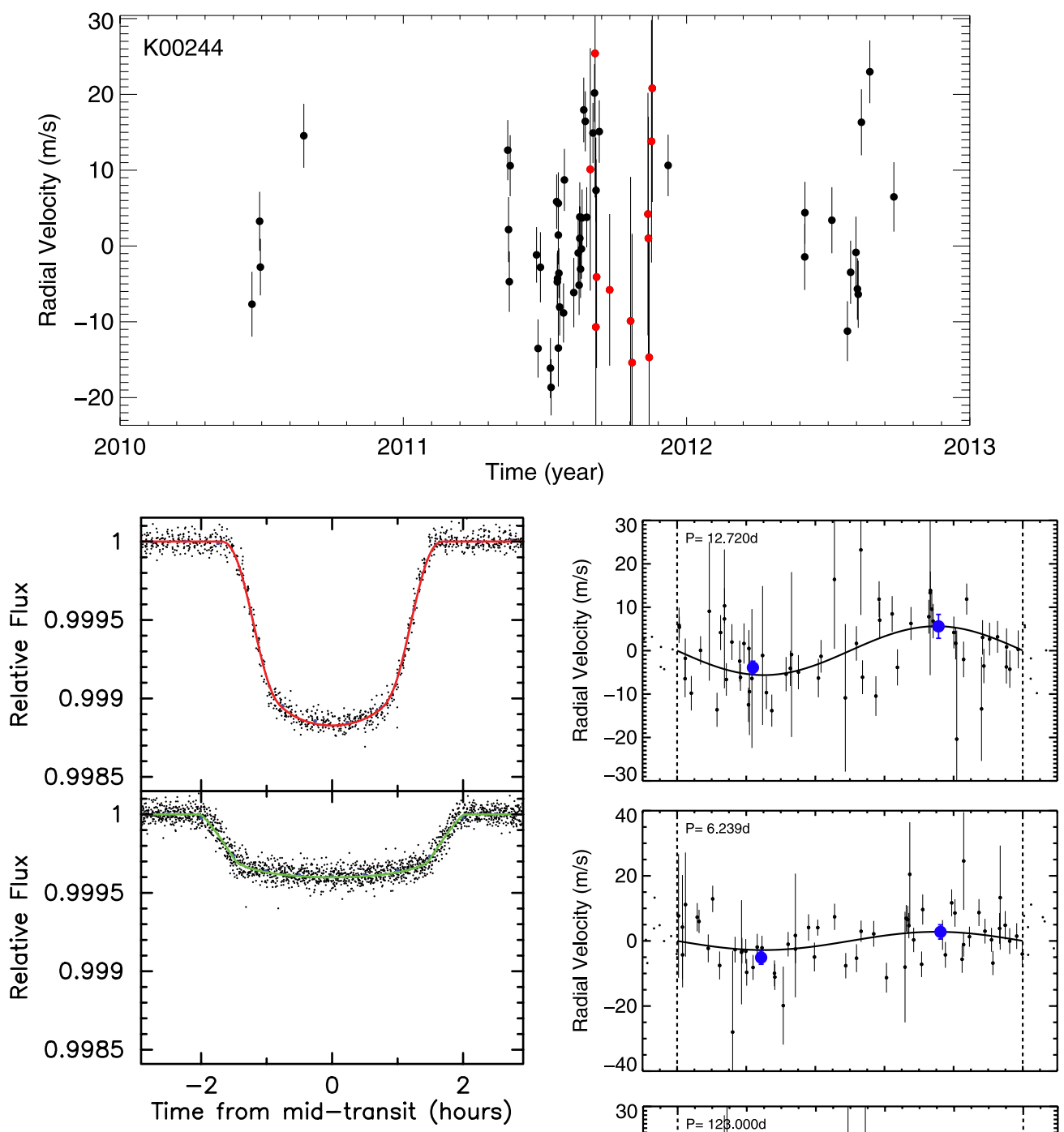

Non-Transiting
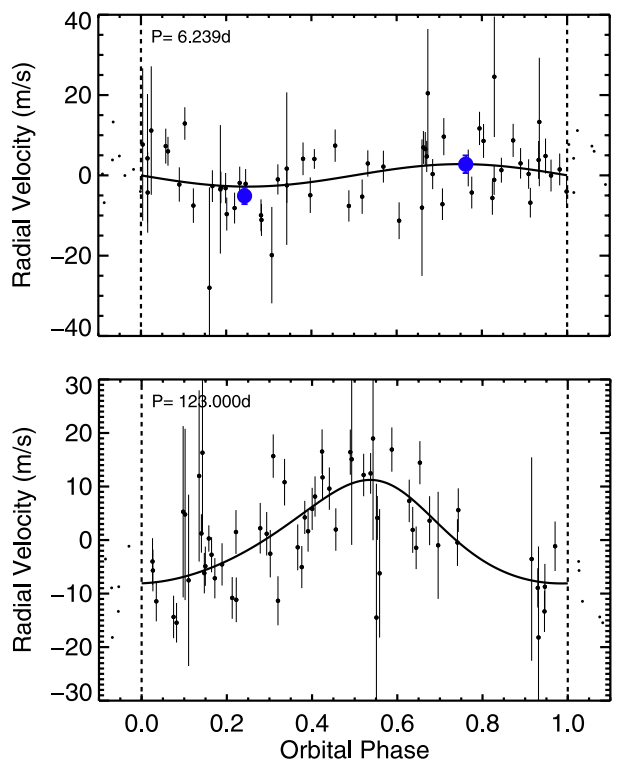

Figure 22. Top: RV vs. time for Kepler-25 (KOI-244). The red points are from the SOPHIE spectrometer at Obs. de Haute-Provence. Lower left: phase-folded Kepler photometry for each planet. The non-transiting planet has no light curve. Lower right: phase-folded radial velocities for each planet. The blue points represent binned RVs near quadrature, same as Figure 2. Kepler-25c: $\mathrm{Rp}=5.20 \pm 0.09 R_{\oplus}, \mathrm{Mp}=24.60 \pm 5.7 M_{\oplus}$. Kepler-25b: Rp $=2.71 \pm 0.05 R_{\oplus}, M p=9.6 \pm 4.2 M_{\oplus}$. Kepler-25d: $\mathrm{Rp}=\mathrm{NA}, \mathrm{Mp}=89.90 \pm 13.7 M_{\oplus}$, period $=123 \pm 2$ days. The 123 days period has an alias at 93 days. Each period provides an equally good fit.

(A color version of this figure is available in the online journal.)

a few arcsec of the target star. The non-detections were taken into account, along with the exclusion radius, in the calculation of the FPP using the method of Morton (2012).

For all 22 KOIs in this paper, we have obtained AO imaging and speckle interferometry. Figures 1-43 (middle panel) show the detectability thresholds for companion stars to all $22 \mathrm{KOIs}$ from these two techniques. The $\mathrm{AO}$ and speckle techniques typically rule out stellar companions as close as $\sim 0^{\prime \prime} 1$ of the target star (especially for Keck AO), depending on wavelength and technique (see Figures 2-44, bottom panel). The spectroscopic technique (see below) becomes effective for companions located closer than $\sim 0$ ". 4 (half of the slit width), complementing AO and speckle. Thus this suite of techniques offers good coverage of companion stars located at a wide range of orbital separations, except for 5-20 au (RV offset too small to support spectral separation, angular offset too small for AO or Speckle to resolve) within which the techniques are not robust at the typical distances of these targets of 100-200 pc. All of the non-detections of stellar companions contributed to the FPP values listed in Table 3.

False positives can be caused by a background eclipsing binary or by star spots (Buchhave et al. 2011; Queloz et al. 

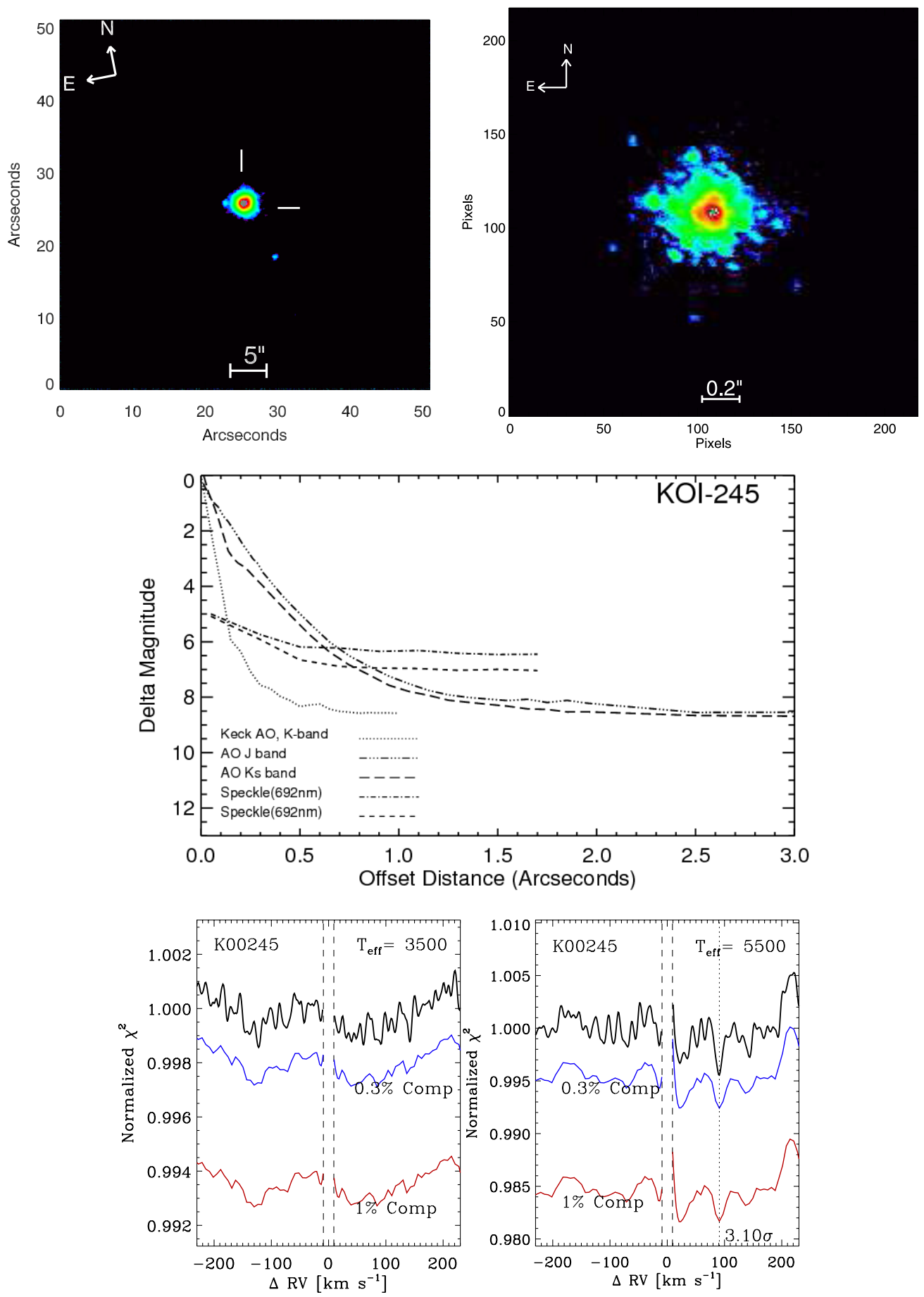

Figure 23. Upper left: seeing-limited image for Kepler-37 (KOI-245). Upper right: adaptive optics image. Middle: limiting magnitudes of companion stars found with Speckle and AO imaging. Lower left and lower right: same as Figure 1, but for Kepler-37. No companions are detected. Any companion brighter than $0.3 \%$ the brightness ( $V$ band) of the primary would have been detected.

(A color version of this figure is available in the online journal.)

2001). Such effects cause the profiles of the absorption lines from the observed composite spectrum to vary in shape as a function of "orbital" phase. We searched for changes in the shapes of line profiles by computing the usual "line bisector," i.e., the relative Doppler shift of the profile near the line core to that in the wings. For all $22 \mathrm{KOIs}$, the line bisectors varied by no more than the noise $\left(\sim 30 \mathrm{~m} \mathrm{~s}^{-1}\right)$ and were not correlated in time with the observed RV variations. Thus, we rule out all eclipsing binaries and star spots that would have caused such bisector changes.

To further aid in constraining potential stellar false-positive scenarios, we also evaluate whether there is a linear trend present in the RV data. A hierarchical triple system, for example, would likely cause the primary to display a long-term acceleration, so the absence of a trend would help rule out these scenarios. We find that all the RV time series have linear trends less than 

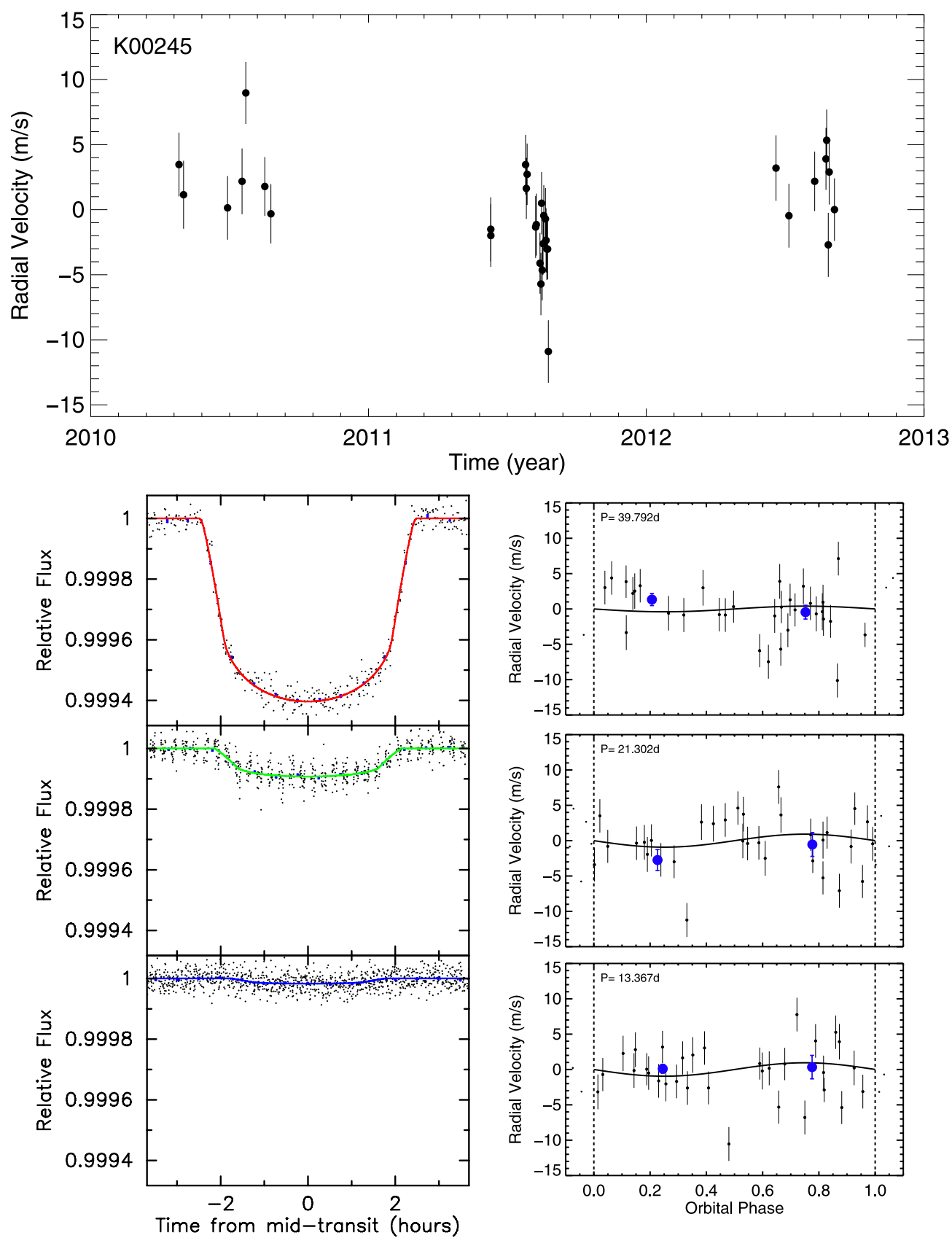

Figure 24. Top: RV vs. time for Kepler-37 (KOI-245). Lower left: phase-folded Kepler photometry for each planet. Lower right: phase-folded radial velocities for each planet. The blue points represent binned RVs near quadrature, same as Figure 2 . Kepler-37d: Rp $=1.94 \pm 0.06 R_{\oplus}, \mathrm{Mp}=1.87 \pm 9.08 M_{\oplus}$. Kepler-37c: Rp $=$ $0.75 \pm 0.03 R_{\oplus}, \mathrm{Mp}=3.35 \pm 4.0 M_{\oplus}$. Kepler-37b: $\mathrm{Rp}=0.32 \pm 0.02 R_{\oplus}, \mathrm{Mp}=2.78 \pm 3.7 M_{\oplus}$.

(A color version of this figure is available in the online journal.)

$5 \mathrm{~m} \mathrm{~s}^{-1}$ amplitude over the course of observation for all KOIs except Kepler-93, Kepler-97, and Kepler-407 which have trends of 39,11 , and $300 \mathrm{~m} \mathrm{~s}^{-1}$ amplitude. The slight curvature in the RVs for Kepler-407 allow us to place limits on the mass and period of the companion.

The FPP calculations included the detectability of physically close-in $(<5 \mathrm{au})$ companion stars to the target star. We analyzed the high-resolution $(R=60,000)$, high signal-to-noise $(\mathrm{S} / \mathrm{N} \approx 150)$ optical spectra of all 22 KOIs for the presence of absorption lines from any second star besides the identified Kepler target star, as described and tested in detail in R. Kolbl \& G. Marcy (2014, in preparation). In brief, the entrance slit of the Keck-HIRES spectrometer had a width of 0.'87, allowing the light from any neighboring stars located within 0'4 to enter the slit. This offers detectability of companion stars complementary to that of $\mathrm{AO}$ and speckle interferometry. The algorithm fits the observed spectrum with the closest-matching member (in a chisquare sense) of our library of 640 AFGKM-type spectra stored on disk, spanning a wide range of $T_{\text {eff }}, \log g$, and metallicities. After proper Doppler shifting, artificial rotational broadening, continuum normalization, and also flux dilution (due to a possible secondary star), that best-fitting primary star spectrum is subtracted from the observed spectrum.

The code then takes the residuals to that spectral fit and performs the same chi-squared search for a "second" spectrum that best fits those residuals. This approach stems from an 

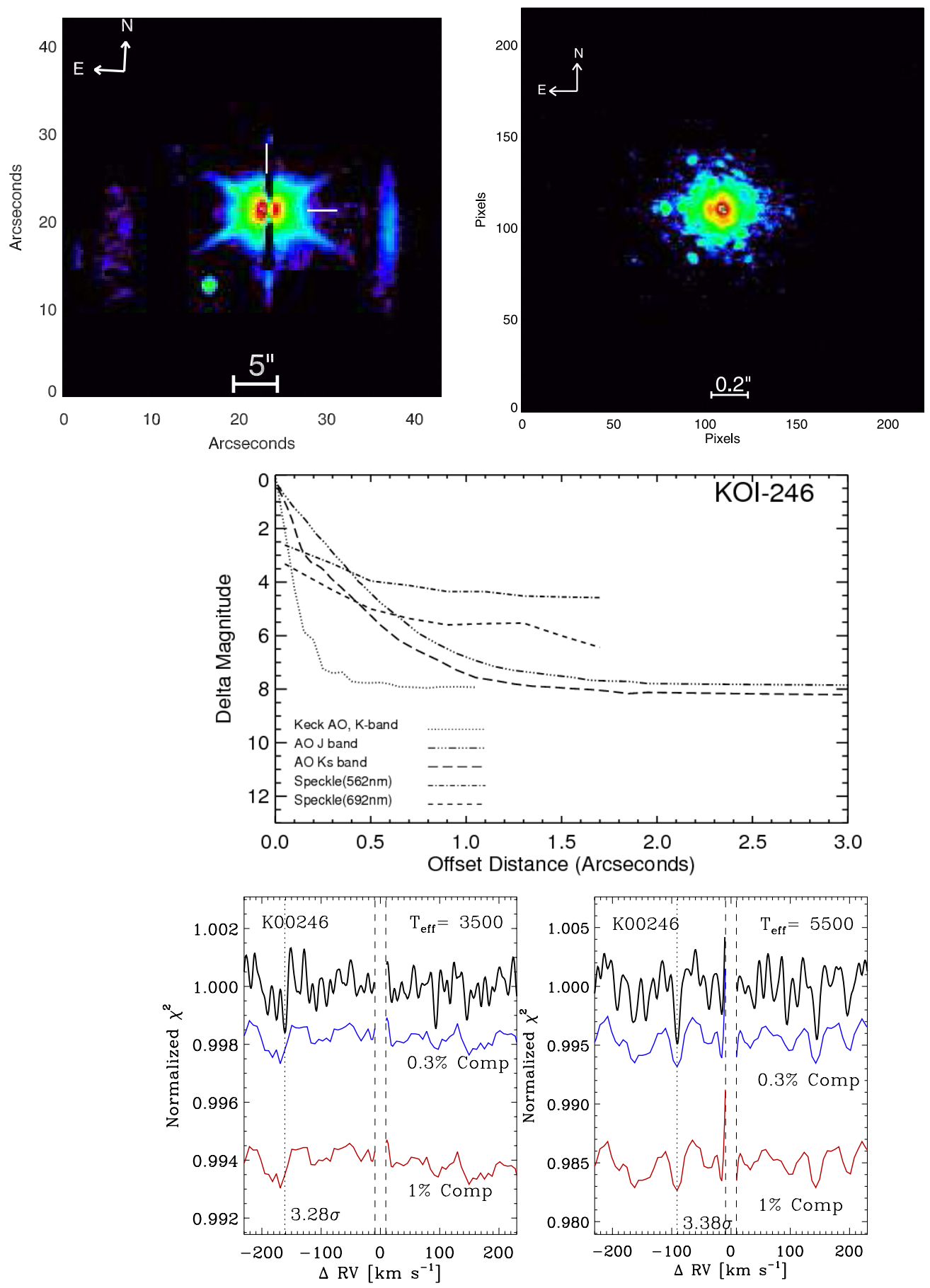

Figure 25. Upper left: seeing-limited image for Kepler-68 (KOI-246). Upper right: adaptive optics image. Middle: limiting magnitudes of companion stars found with Speckle and AO imaging. Lower left and lower right: same as Figure 1, but for Kepler-68. No companions are detected. Any companion brighter than $0.5 \%$ the brightness ( $V$ band) of the primary would have been detected.

(A color version of this figure is available in the online journal.)

Occam's razor perspective, rather than immediately doing a self-consistent two-spectrum fit. If one spectrum adequately fits the spectrum, without "need" to invoke a second spectrum, then the spectrum can only be deemed single. A low value of chi-squared for the fit of any library spectrum (indeed a subset of them) to the residuals serves to indicate the presence of a second spectrum. We establish a detection threshold by injecting fake spectra into the observed spectrum and executing the algorithm above to determine the value of chi-square for any relative Doppler shift, $\Delta R V$ between the companion star and the primary star that causes a $3 \sigma$ detection of the secondary star. Figures 1-43 (bottom panels) show the resulting plot of chi-squared versus $\Delta R V$ in search of a clear minimum that would signify the presence of a second spectrum. The blue and red lines show how low chi-square would be for a companion having $0.3 \%$ and $1.0 \%$, respectively, of the optical flux of the primary, based on the injection of fake secondary spectra. None of the 22 KOIs shows evidence of a second star within 0.4 , at flux thresholds of $\sim 0.3 \%$ of the flux of the primary star. There is a blind spot for $\Delta R V<10 \mathrm{~km} \mathrm{~s}^{-1}$ for which 

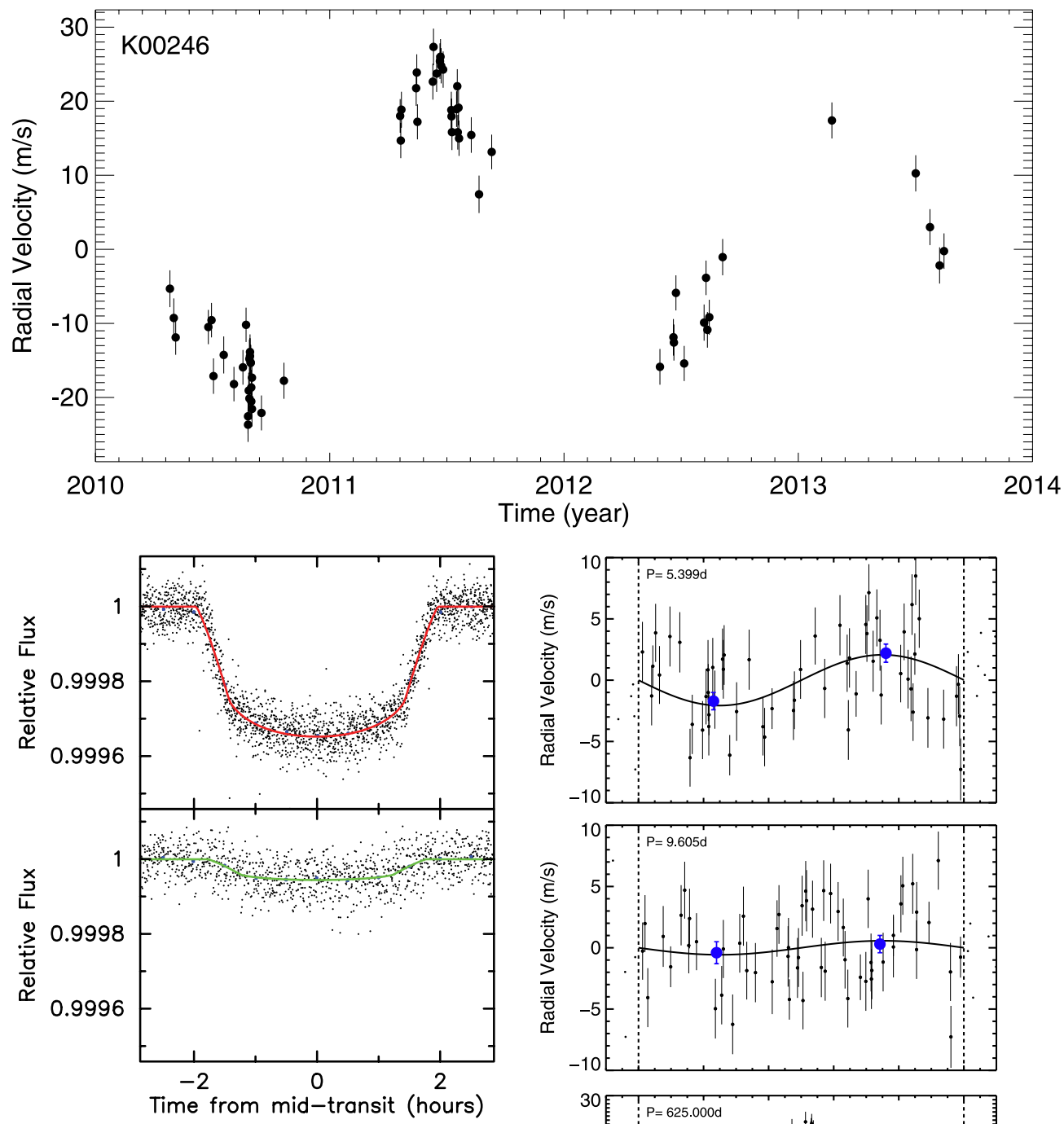

Non-Transiting
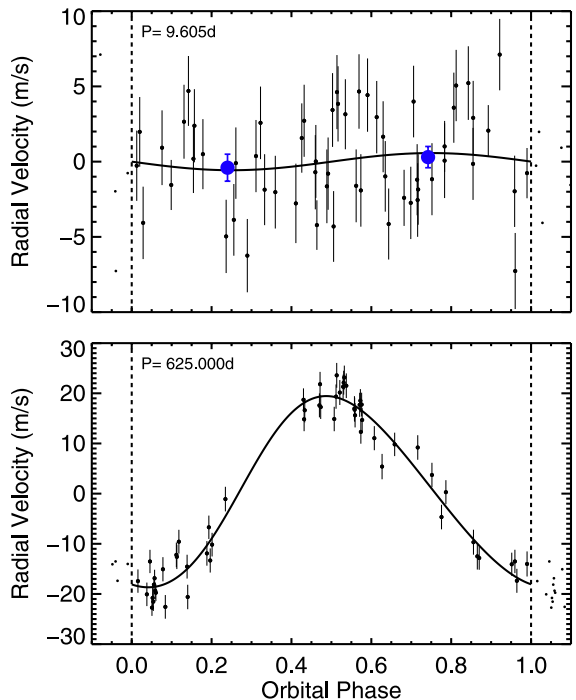

Figure 26. Top: RV vs. time for Kepler-68 (KOI-246). Lower left: phase-folded Kepler photometry for each planet. Lower right: phase-folded radial velocities for each planet. The blue points represent binned RVs near quadrature, same as Figure 2. Kepler-68b: Rp $=2.33 \pm 0.02 R_{\oplus}, \mathrm{Mp}=5.97 \pm 1.7 M_{\oplus}$. Kepler-68c: Rp $=$ $1.00 \pm 0.02 R_{\oplus}, \mathrm{Mp}=2.18 \pm 3.5 M_{\oplus}$. Kepler-68d: Rp $=\mathrm{NA}, \mathrm{Mp}=267 \pm 16 M_{\oplus}$, period $=625 \pm 16$.

(A color version of this figure is available in the online journal.)

the absorption lines overlap, preventing effective detection of any companion stars. Thus, companion stars orbiting inward of $\sim 5$ au are detectable by this technique, but companions farther out will have too small a $\Delta R V$ to be seen. Even for companions orbiting within $\sim 5$ au, the orbital phase might result in a RV less than $10 \mathrm{~km} \mathrm{~s}^{-1}$ relative to the primary star. Secondary stars with rotational $v \sin i>10 \mathrm{~km} \mathrm{~s}^{-1}$ suffer from degraded detectability due to enhanced line broadening. Normal FGKM secondaries only rarely have high $v \sin i$ except tidally locked close binaries. Similarly, secondary stars with spectral types earlier than F5 or white dwarfs have few spectral lines and suffer from poor detectability. We note that short period stellar binaries are unlikely given the clean transit signature and the dynamical instability of the planet that would result.

Four KOIs were also observed with the lucky imaging technique, namely Kepler-100, Kepler-102, Kepler-37, and Kepler-409. We used the lucky imaging camera AstraLux Hormuth (2007) mounted at the $2.2 \mathrm{~m}$ telescope at Calar Alto Observatory (Almería, Spain). Good quality on-site seeing of $0 ! 7-0 ! 9$ during the observations combined with short exposure times lead to diffraction-limited images of the four targets in a $24 \times 24 \mathrm{arcsec}$ field of view. A total of 30,000 frames of $0.030 \mathrm{~s}$ each were acquired for Kepler-37 and Kepler-409, and 40,000 frames were acquired for Kepler- 100 (with $0.083 \mathrm{~s}$ of exposure time) and Kepler-102 ( $\left.t_{\exp }=0.068 \mathrm{~s}\right)$. The basic reduction, 

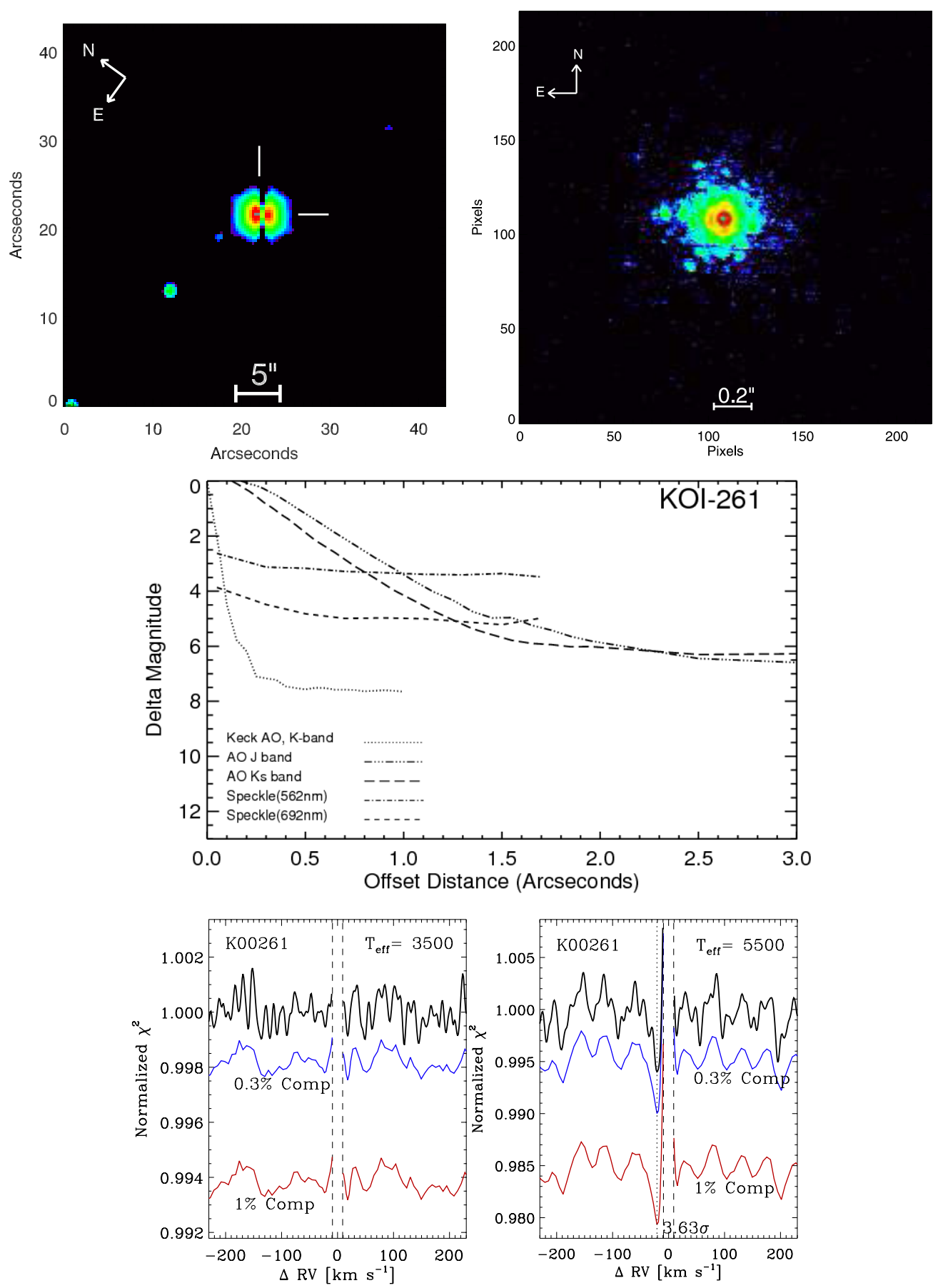

Figure 27. Upper left: seeing-limited image for Kepler-96 (KOI-261). Upper right: adaptive optics image. Middle: limiting magnitudes of companion stars found with Speckle and AO imaging. Lower left and lower right: same as Figure 1, but for Kepler-96. No companions are detected. Any companion brighter than 0.5\% the brightness ( $V$ band) of the primary would have been detected.

(A color version of this figure is available in the online journal.)

frame selection and image combination were carried out with the AstraLux pipeline ${ }^{47}$ (Hormuth 2007). During the reduction process, the images are resampled from the original pixel scale of 47.18 mas pixel $^{-1}$ to 23.59 mas pixel $^{-1}$. The plate scale was measured with the ccmap package of IRAF by matching the XY positions of 66 stars identified in an AstraLux image with their counterparts in the Yanny et al. (1994) catalog of the Hubble Space Telescope. The results from Lucky imaging were not used in the false alarm probability calculations.

\footnotetext{
47 www.mpia.mpg.de/ASTRALUX
}

We computed the sensitivity curves of our reduced images with a $10 \%$ of selection rate (which optimizes the instrument/telescope configuration), and obtained the limiting magnitudes at different angular separations (see additional details in Lillo-Box et al. 2012). The specific limits for each observed KOI are shown in Figures 1-43 (bottom panels). Thus, we can assure that no objects are found within such sensitivity limits.

Light curves in the infrared may also be used to inform FPPs (Cochran et al. 2011; S. Ballard et al. 2014, in preparation) (Désert 2014), but we did not use them here, deferring such 

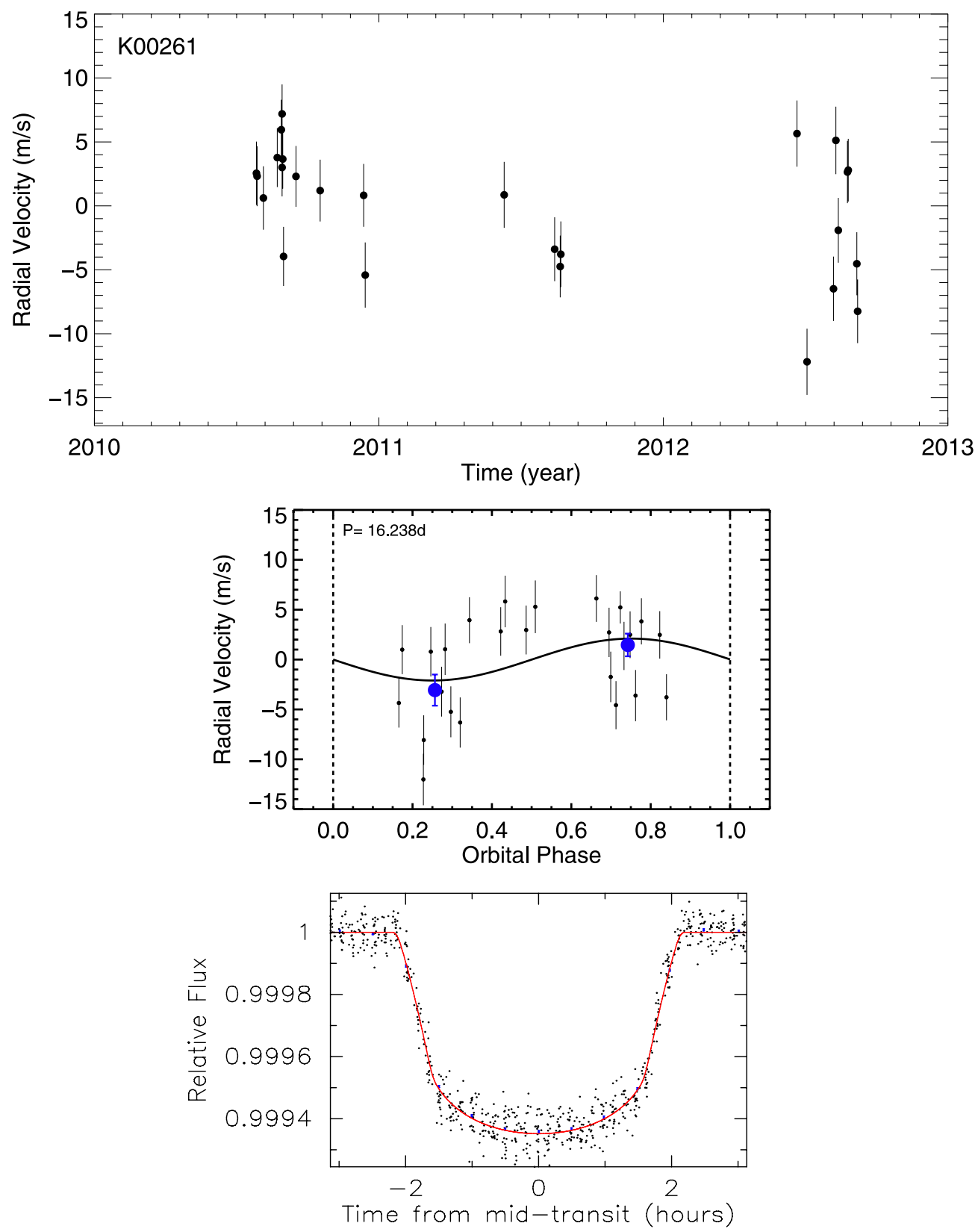

Figure 28. Top: RV vs. time for Kepler 96 (KOI-261). Middle: phase-folded radial velocities for each planet. Bottom: phase-folded Kepler photometry for the planet. The blue points represent binned RVs near quadrature, same as Figure 2. Kepler 96b: $R p=2.67 \pm 0.22 R_{\oplus}, \mathrm{Mp}=8.46 \pm 3.4 M_{\oplus}$.

(A color version of this figure is available in the online journal.)

analysis for later papers (i.e., S. Ballard et al. 2014, in preparation and S. Ballard 2013, private communication). These additional constraints that often rule out some false-positive scenarios serve to reduce the FPP below that reported formally here.

\subsection{Computing Formal False-positive Probabilities}

For each planet candidate presented in this paper, we incorporate all these follow-up constraints-imaging, spectroscopic analysis, and RV trend analysis-into the FPP-calculating procedure described in detail in Morton (2012). This procedure combines information about predicted occurrence rates and distributions of false positives with the observed shape of the light curve and follow-up observations in order to calculate the relative probabilities of an observed signal being by a caused by true planet or any of a number of false-positive scenarios.
The analysis parameterizes each phase-folded dimming profile with three parameters, its duration, depth, and the ratio of the total duration to the duration of "ingress" and "egress," using the geometrical approximation of a trapezoid. The distribution of properties of the stars and their companions (stellar or planetary) toward the target star within the Milky Way Galaxy inform the probabilities of all the false-positive scenarios by using their corresponding light curves, also approximated as trapezoids.

In addition to the follow-up observations and analysis discussed above, these FPP calculations also take into account detailed measurements of an angular "exclusion radius." The maximum possible angular distance from the target star that a false-positive-causing dimming star could be while remaining consistent with the lack of astrometric displacement detected 

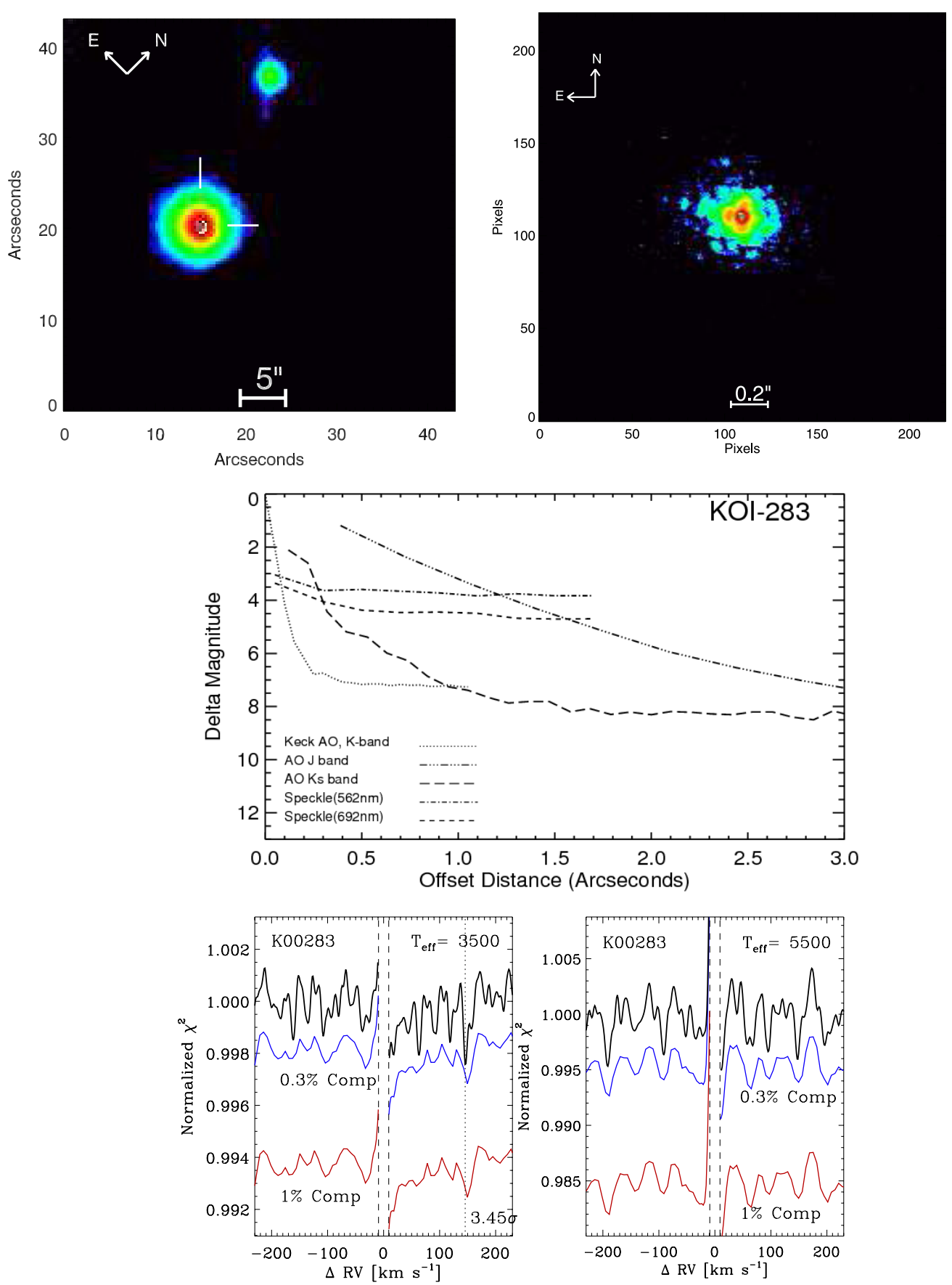

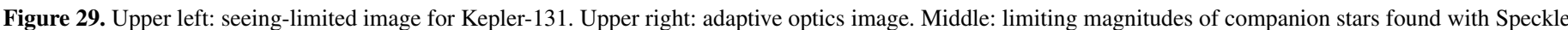

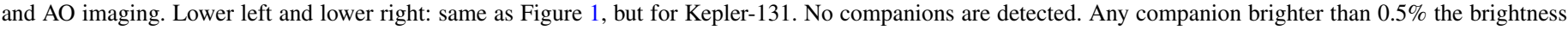
( $V$ band) of the primary would have been detected.

(A color version of this figure is available in the online journal.)

between the times in and out of "transit." Any neighboring stars within the photometric aperture that both dim adequately to cause the observed overall dimming and are located farther than this exclusion radius from the Kepler target star would cause an astrometric shift in photometric difference image centroid position between times "in-transit" and "out-of-transit." The Kepler Project DV process routinely checks for such displacements, ruling them false positives.

We have carefully measured this maximum exclusion radius for all stars using the method described in detail by Bryson et al. (2013). The exclusion radii are listed in Table 3 in Column 2, in arcsec from the target star. The exclusion radii range from $0^{\prime \prime} 01-4^{\prime \prime}$, with a median value of 0.30 . For 10 bright target stars, namely Kepler-100, 93, 102, 25, 37, 68, 96, 131, 408, and 409 no formal exclusion radius could be computed due to saturation of the Kepler CCD detector. For them, a reasonable exclusion radius of $4^{\prime \prime}$ was adopted corresponding to a position displacement of a full Kepler pixel that can be excluded despite the saturation.

The exclusion radius for each of the 22 KOIs establishes a circular area on the sky (i.e., a solid angle) centered on the target star within which there could be a background star that either is 

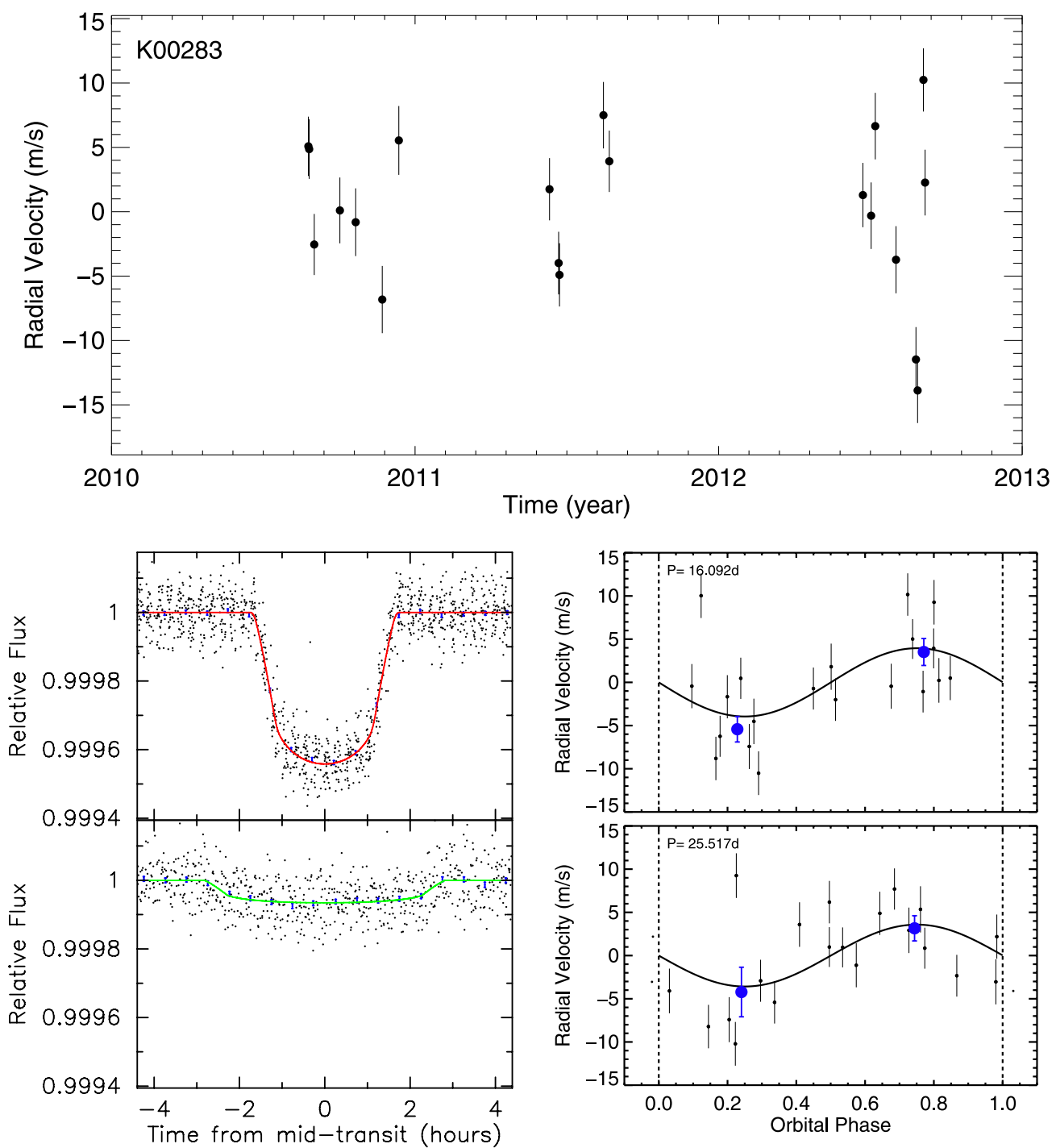

Figure 30. Top: RV vs. time for Kepler-131 (KOI-283). Lower left: phase-folded Kepler photometry for each planet. Lower right: phase-folded radial velocities for each planet. The blue points represent binned RVs near quadrature, same as Figure 2 . Kepler-131b: Rp $=2.41 \pm 0.20 R_{\oplus}, \mathrm{Mp}=16.13 \pm 3.5 M_{\oplus}$. Kepler-131c: Rp $=$ $0.84 \pm 0.07 R_{\oplus}, \mathrm{Mp}=8.25 \pm 5.9 M_{\oplus}$.

(A color version of this figure is available in the online journal.)

an eclipsing binary (BGEB) or has a transiting planet (BGPL), mimicking a transiting planet around the target star. That circular area could also contain an eclipsing binary star gravitationally bound to the target star, constituting a hierarchical triple system (HEB). We also consider the possibility that the target star itself is an eclipsing binary; however, this scenario is completely ruled out for every KOI by the observed lack of large RV variations. Note that for each scenario including some sort of eclipsing binary, we also include eclipsing binaries in elliptical orbits that show only a secondary eclipse.

We list the results of these calculations in Table 3. For each KOI, we list the probability that it is caused by each falsepositive scenario, with their sum being the total FPP. Also included in Table 3 is the assumed planet occurrence rate for each KOI (between $2 / 3 R_{p}$ and $4 / 3 R_{p}$ ) that is a factor in the probability calculations. For probabilities smaller than 0.0001 , we simply state in the table as " $<1 \mathrm{e}-4$, as quoting exact probabilities smaller than this seems unrealistic to us given unavoidable uncertainties in the input assumptions. Table 3 also lists the exclusion radii that are used and whether there was any detected companion, and its separation(s) in the event of detections.

The exclusion radius and false-positive scenarios are further informed by AO imaging and speckle interferometry for all 22 KOIs. The detection thresholds for companions from those two types of observations are shown in Figures 1-44. All companion stars detected are listed in Table 3 in Column 4 and described for individual targets in Section 7. The majority of the target stars have no detected neighboring star in any of our highresolution images, including Keck AO images with resolution of 0 '05 (see Table 3). Such "single" stars with no detected stellar neighbor enjoy a severely limited set of plausible falsepositive scenarios. For those target stars that have a neighboring companion, listed in Table 3 Column 4 , the companions all reside angularly outside the maximum exclusion radius derived from centroid astrometry described above. Thus, those detected companions are not able to explain the transit-like dimming of the target star, supporting the planet hypothesis. We also use the non-detections of companion stars in a spectroscopic search for secondary lines (see the next subsection). 

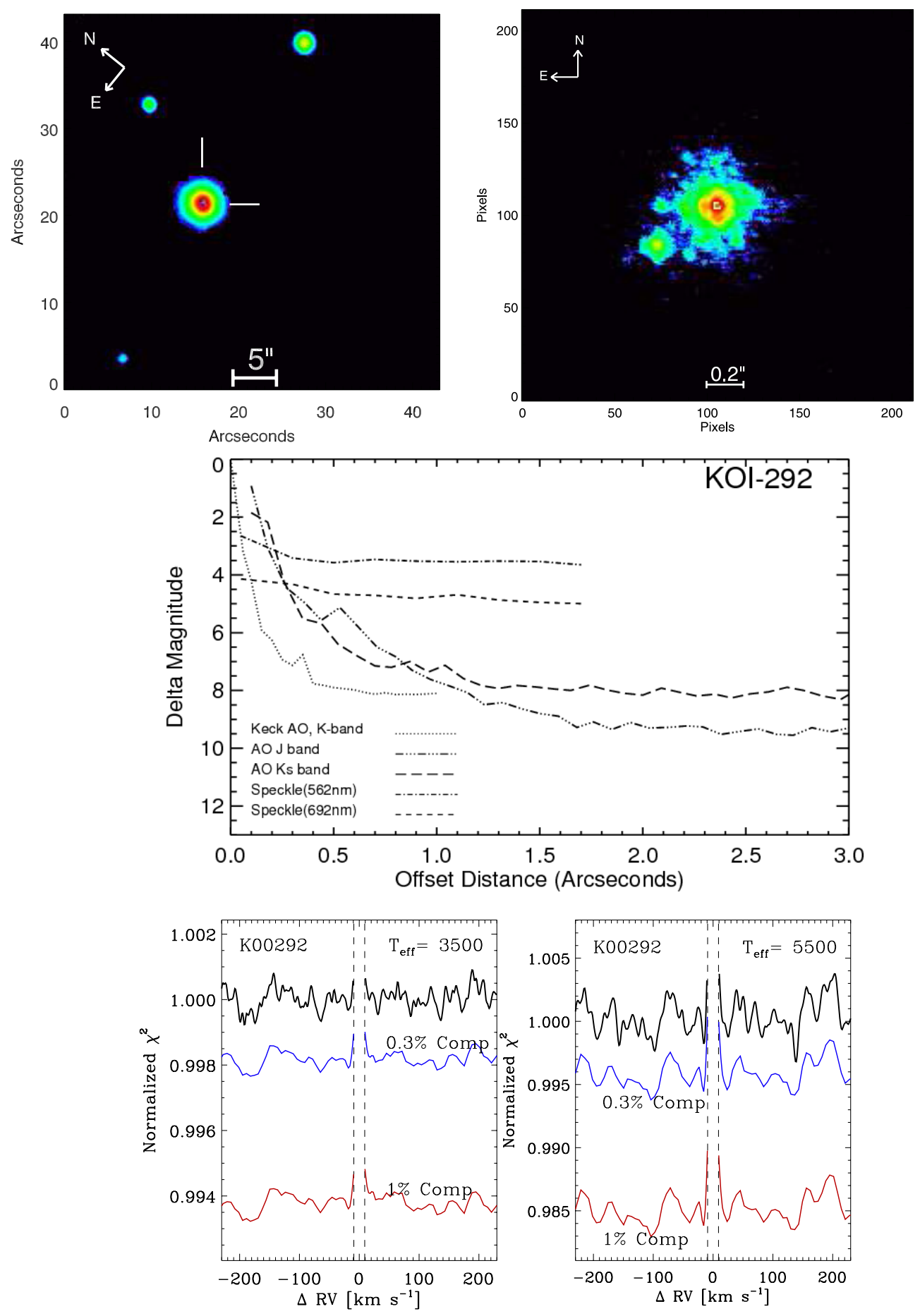

Figure 31. Upper left: seeing-limited image for Kepler-97 (KOI-292). Upper right: adaptive optics image. Note companion 0!'38 to the SE, 3.2 mag fainter in $K$ band $(2.2 \mu)$. The transiting planet could orbit the primary or secondary star. But lack of photo-center displacement suggests the planet orbits the primary star. Middle: limiting magnitudes of companion stars found with Speckle and AO imaging. Lower left and lower right: same as Figure 1, but for Kepler-97. No companions are detected. Any companion brighter than $0.5 \%$ the brightness ( $V$ band) of the primary would have been detected.

(A color version of this figure is available in the online journal.)

Table 3 presents the results of our formal false-positive calculations. The first two columns gives the name of the planets. The third column gives the angular exclusion radius described above. The fourth column gives the angular separation of any neighboring stars detected by AO, speckle imaging, or seeinglimited imaging. When no such neighbor was found, Column 4 contains "single." The fifth column gives the probability that the target might itself be an eclipsing binary. All of these probabilities are less than 0.0001 because it is geometrically difficult for a companion star to eclipse the primary star yielding the same short transit duration and small transit depth accomplished by a planet. Columns 6,7 , and 8 give the probabilities that the observed light curve is produced by a gravitationally bound (Hierarchical) eclipsing binary (HEB), a background eclipsing binary (BGEB), and a background star orbited by a planet (BGPL), respectively. Column 9 gives the 

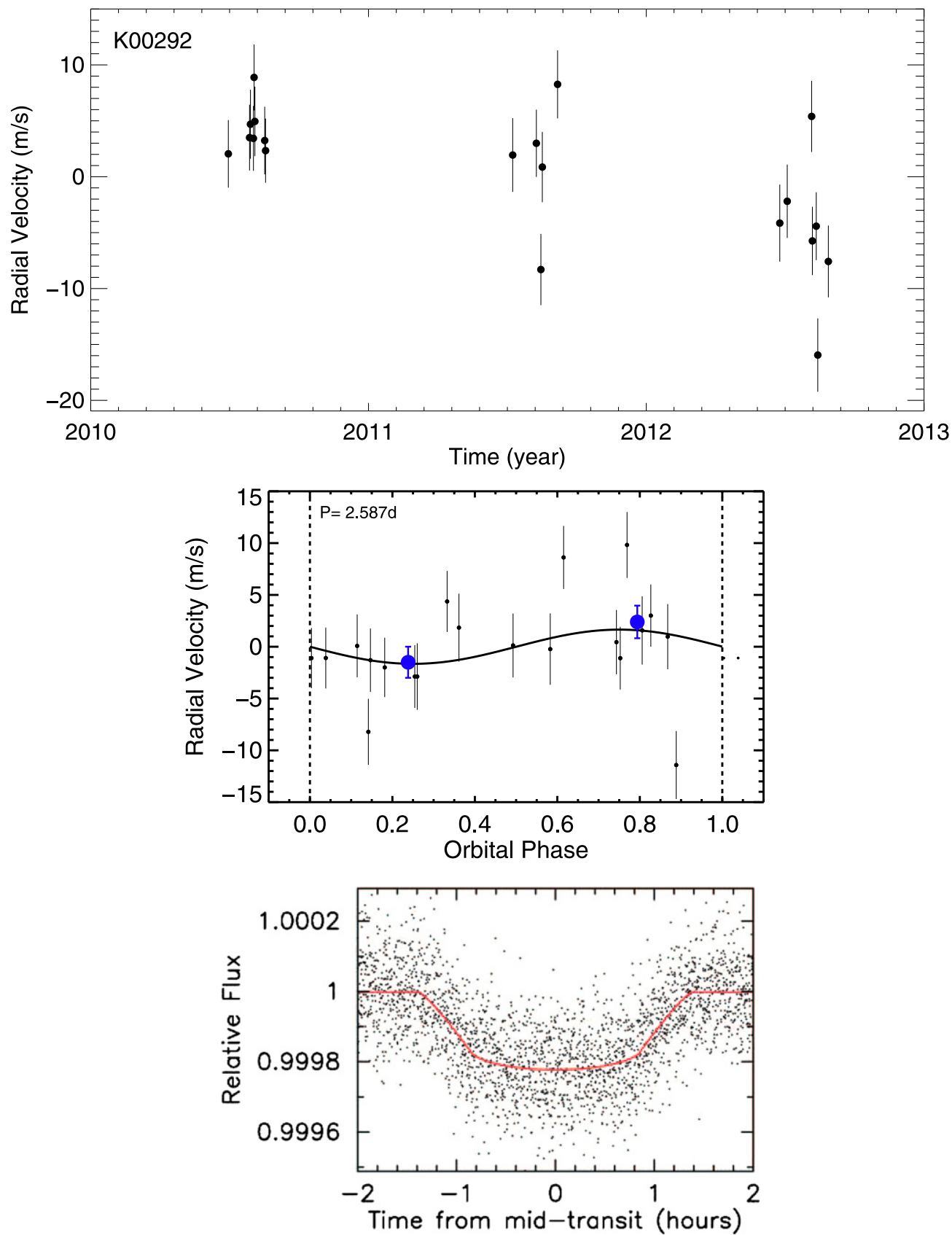

Figure 32. Top: RV vs. time for Kepler-97 (KOI-292). Middle: phase-folded radial velocities for each planet. Bottom: phase-folded Kepler photometry for each planet. The blue points represent binned RVs near quadrature, same as Figure 2. Kepler-97b: Rp $=1.48 \pm 0.13 R_{\oplus}, \mathrm{Mp}=3.51 \pm 1.9 M_{\oplus} . \mathrm{KOI}-292.10: \mathrm{Rp}=\mathrm{NA}, \mathrm{Mp}=$ $>789 M_{\oplus}$, period $>344$.

(A color version of this figure is available in the online journal.)

estimated prior probability for the candidate planet within a $30 \%$ range in period and size. This $30 \%$ is arbitrary and not particularly conservative. But the resulting blend probabilities are so small for the planets here that increasing the radius range would have little effect on the conclusions about the validation of the planets, except for the two cases with FPP above $2 \%$, which would incur an increased FPP.

Column 10 gives the sum of the FPPs (in Columns 5-8), constituting the final FPP. More precisely, the FPP is the sum of probabilities in Columns 5-8 divided by the sum of those columns plus the probability that it's a planet, which is $1-\mathrm{FPP} \approx 1$.

The FPPs are less than $1 \%$ for all transiting planet candidates here, except for KOI-1612.01, which has a FPP of 0.021, respectively, as shown in Column 9 of Table 3. We thus find 41 transiting planets to be formally "validated" as highly likely to be real, with an FPP less than 1\%. The remaining planet candidate, KOI-1612.01, has an FPP of $2.1 \%$, making that planet's existence highly likely also, but not satisfying the strict $1 \%$ FPP criterion.

We note here that Kepler-93, Kepler-97, and Kepler-407 have detected linear trends. The FPP calculations whose results are presented in the table are based on the assumption that there is no detected linear trend up to the limit provided $\left(<5 \mathrm{~m} \mathrm{~s}^{-1}\right.$ over the relevant time baselines for all except these three). The fact that these three systems have detected trends means there is a companion there, which changes the probabilities involved in the calculation in a way that has not been quantified. However, 

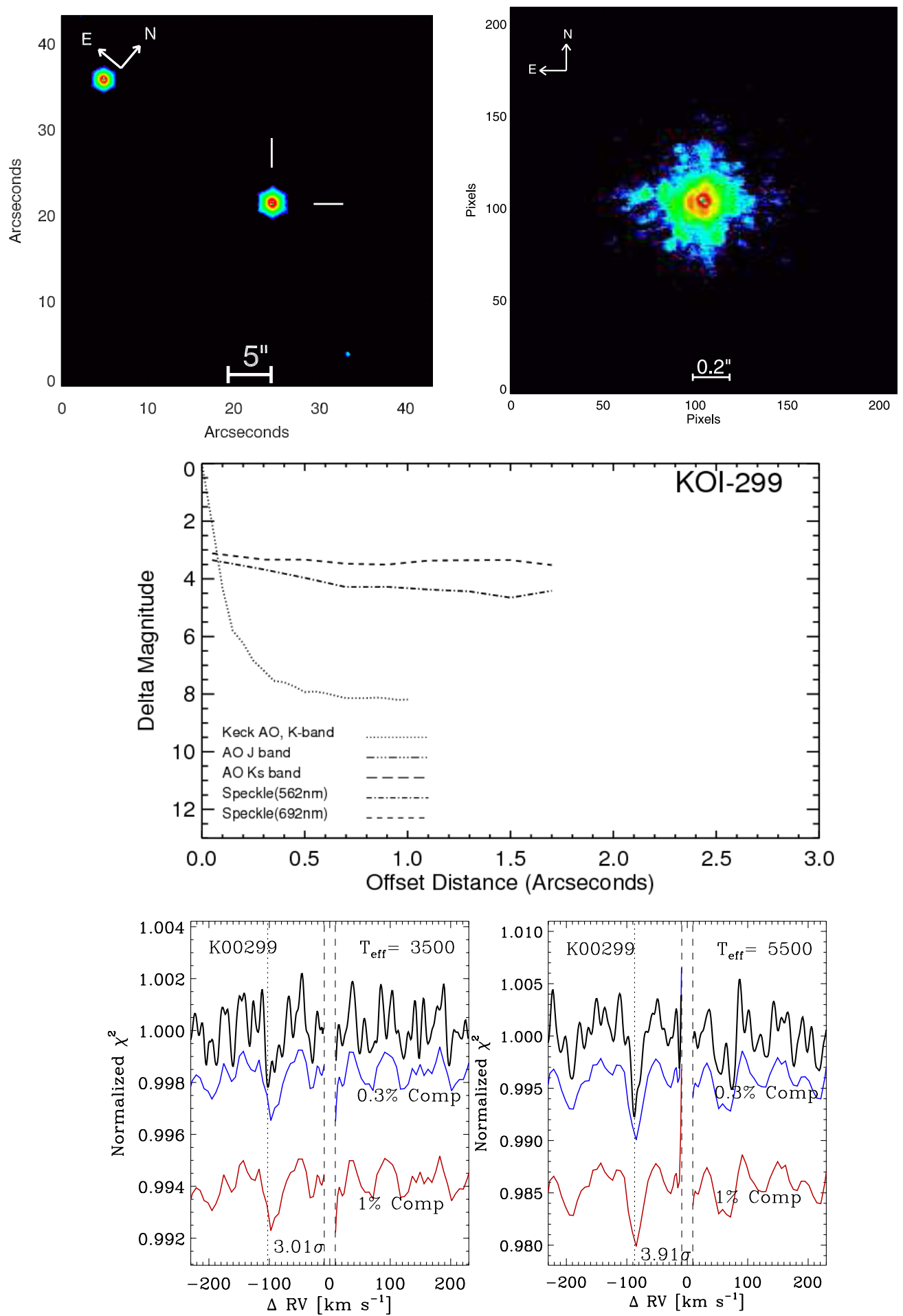

Figure 33. Upper left: seeing-limited image for Kepler-98 (KOI-299). Upper right: adaptive optics image. Middle: limiting magnitudes of companion stars found with Speckle and AO imaging. Lower left and lower right: same as Figure 1, but for Kepler-98. No companions are detected. Any companion brighter than $0.5 \%$ the brightness ( $V$ band) of the primary would have been detected.

(A color version of this figure is available in the online journal.)

the FP scenario that would get a boost from this is the HEB scenario, whose likelihood is quite small already for all three of these systems. Thus even a large prior boost for the HEB scenario would be unlikely to qualitatively change the results.

In addition, Kepler-97 has a close companion detected at just outside the exclusion radius provided by the centroid analysis. This changes the false-positive calculation in a similar manner, meaning the priors for the various false-positive scenarios will be higher, as there is a known companion present. However, given that there is also a detected RV trend in Kepler-97, this close companion is likely to be physically associated, meaning the HEB scenario should receive the prior boost rather than the BGEB or BGPL. And as mentioned above, the HEB scenario has a low likelihood for this KOI (i.e., the signal shape is not 

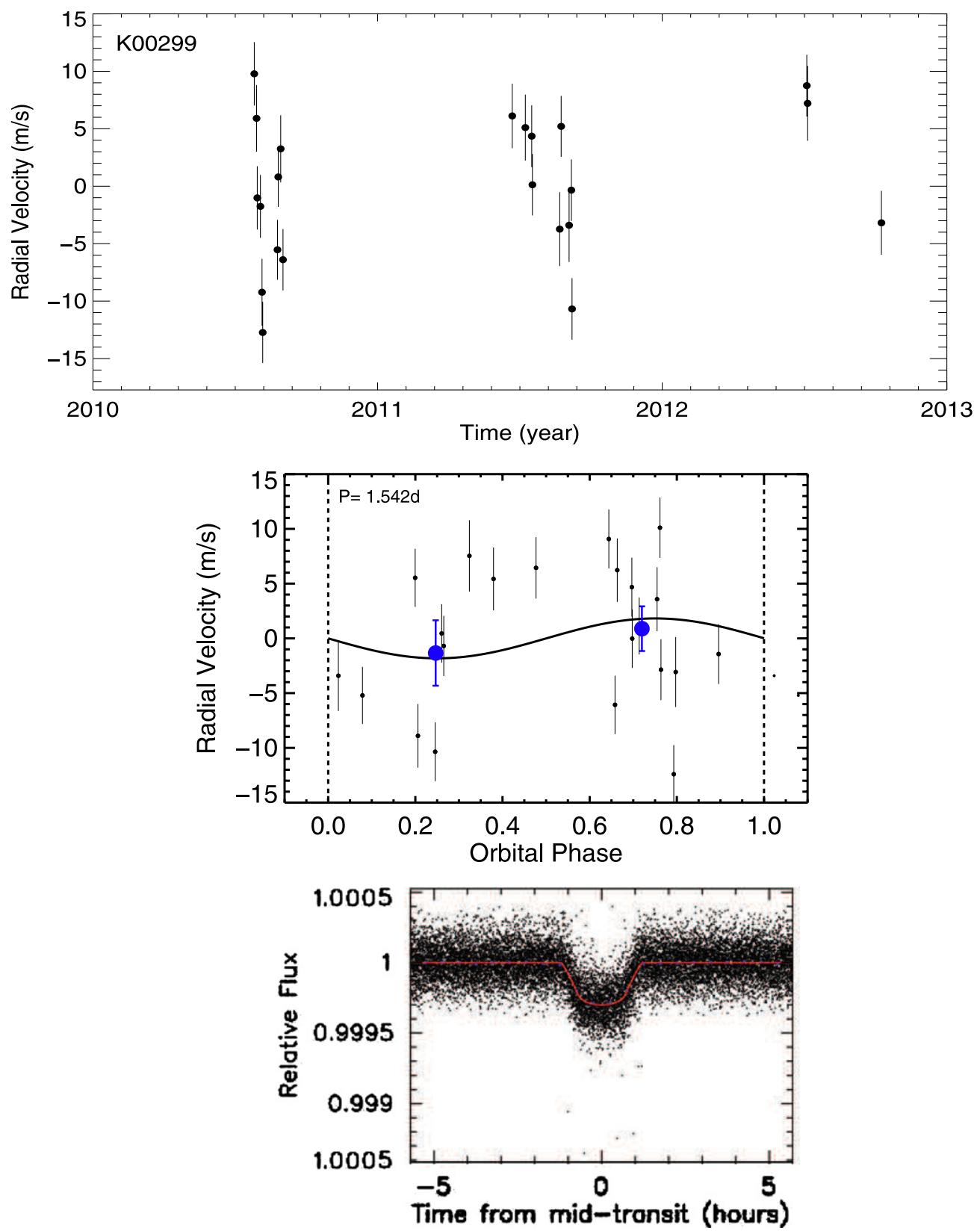

Figure 34. Top: RV vs. time for Kepler-98 (KOI-299). Middle: phase-folded radial velocities for each planet. Bottom: phase-folded Kepler photometry for the transiting planet. The blue points represent binned RVs near quadrature, same as Figure 2 . Kepler-98b: $\mathrm{Rp}=1.99 \pm 0.22 R_{\oplus}, \mathrm{Mp}=3.55 \pm 1.6 M_{\oplus}$.

(A color version of this figure is available in the online journal.)

compatible with the vast majority of simulated HEBs). As a result, we still validate all three of these planets, though with the caveat that the true FPPs of these systems have not been as confidently quantified as those for which neither a trend nor a companion was detected.

\subsection{Gravitationally Bound Stars with Transiting Planets}

In our calculation of the FPP we do not deem as false positives those scenarios that involve a gravitationally bound companion star transited by a planet. In such systems a real planet does exist, albeit orbiting an unresolved bound companion star. We have considered carefully whether to deem such planets as "real" or "false positives." We find no easy answer. One useful thought experiment involves a bound companion star that is the nearly the same brightness as the primary star. It makes little sense to deem a planet a "false positive" simply because it orbits a slightly fainter companion star. Indeed, the true planet-host star may be the "secondary star" only in some wavelength bandpasses, thus rendering the planet "real" depending on which bandpass one considers, which is clearly absurd.

Continuing the thought experiment to companion stars that are progressively fainter than the primary star leads to no "break point" at which the planet around that companion should be suddenly deemed a "false positive." Therefore, all transiting planets orbiting any (perhaps unknown) bound star in the stellar system is considered a real "planet." But if a planet does orbit a cooler, smaller secondary star the planet is likely to be larger than was inferred from a model of the planet orbiting the hotter, larger primary star, to yield a predicted transit depth as deep as 

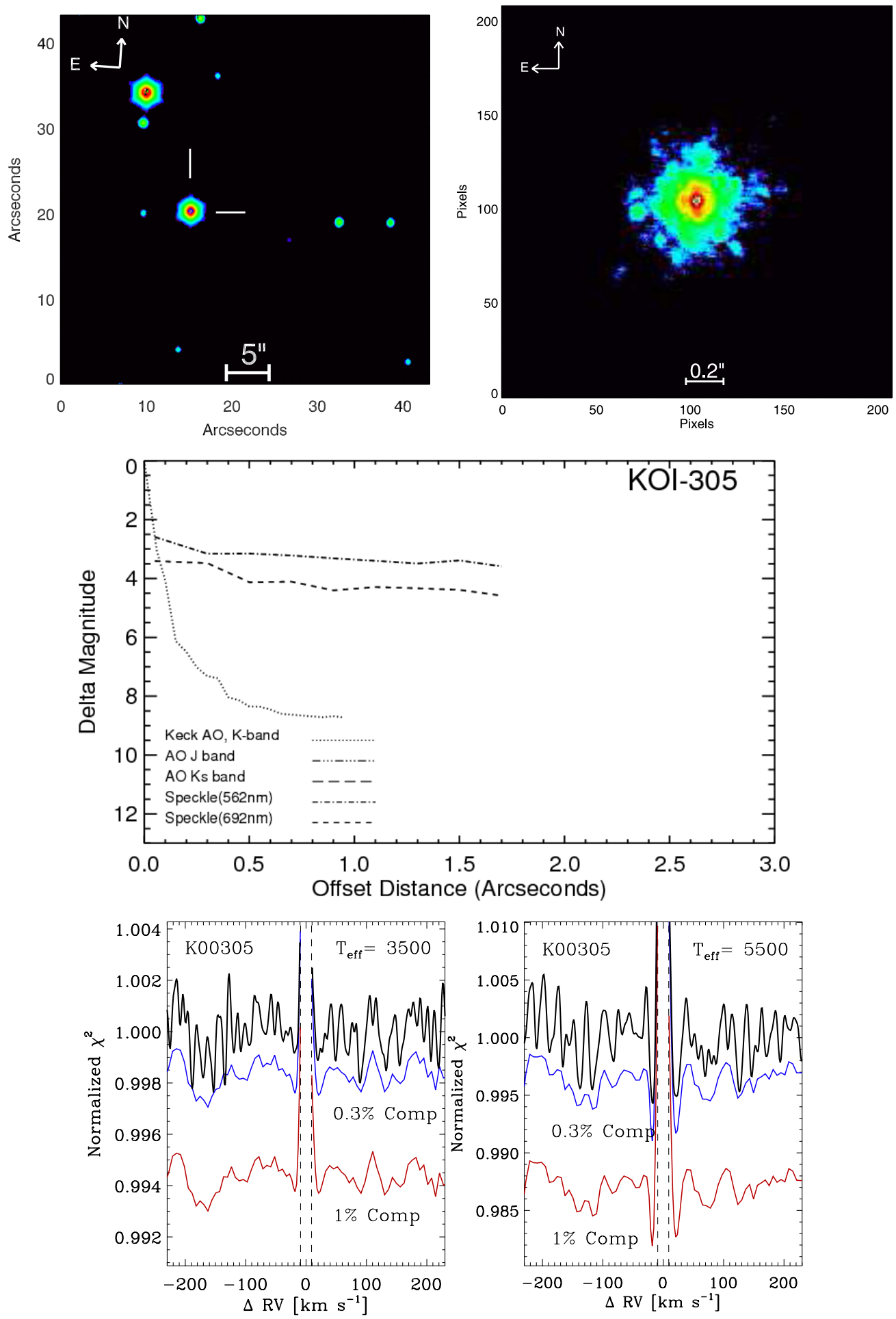

Figure 35. Upper left: seeing-limited image for Kepler-99 (KOI-305). Upper right: adaptive optics image. Middle: limiting magnitudes of companion stars found with Speckle and AO imaging. Lower left and lower right: same as Figure 1, but for Kepler-99. No companions are detected. Any companion brighter than 0.5\% the brightness ( $V$ band) of the primary would have been detected.

(A color version of this figure is available in the online journal.)

that observed. Planet occurrence decreases toward larger sizes (Howard et al. 2012; Fressin et al. 2013; Petigura et al. 2013), and the contribution of light from any secondary star to the photometric aperture is much smaller than that by the primary star. Therefore, the probability that the transit light curve is caused by a larger planet transiting a fainter secondary star in a binary system is less than $50 \%$ and can be estimated, as follows.
Roughly $50 \%$ of FGK stars have a companion star and among those binaries, there is a roughly $50 \%$ probability that the transiting planet orbits the secondary star. Thus the probability that a KOI consists of a transiting planet orbiting a secondary star is $0.5 \times 0.5=0.25$. This reasoning ignores the planet occurrence as a function of host star mass, which could be higher or lower than that of the primary. As mentioned, the 

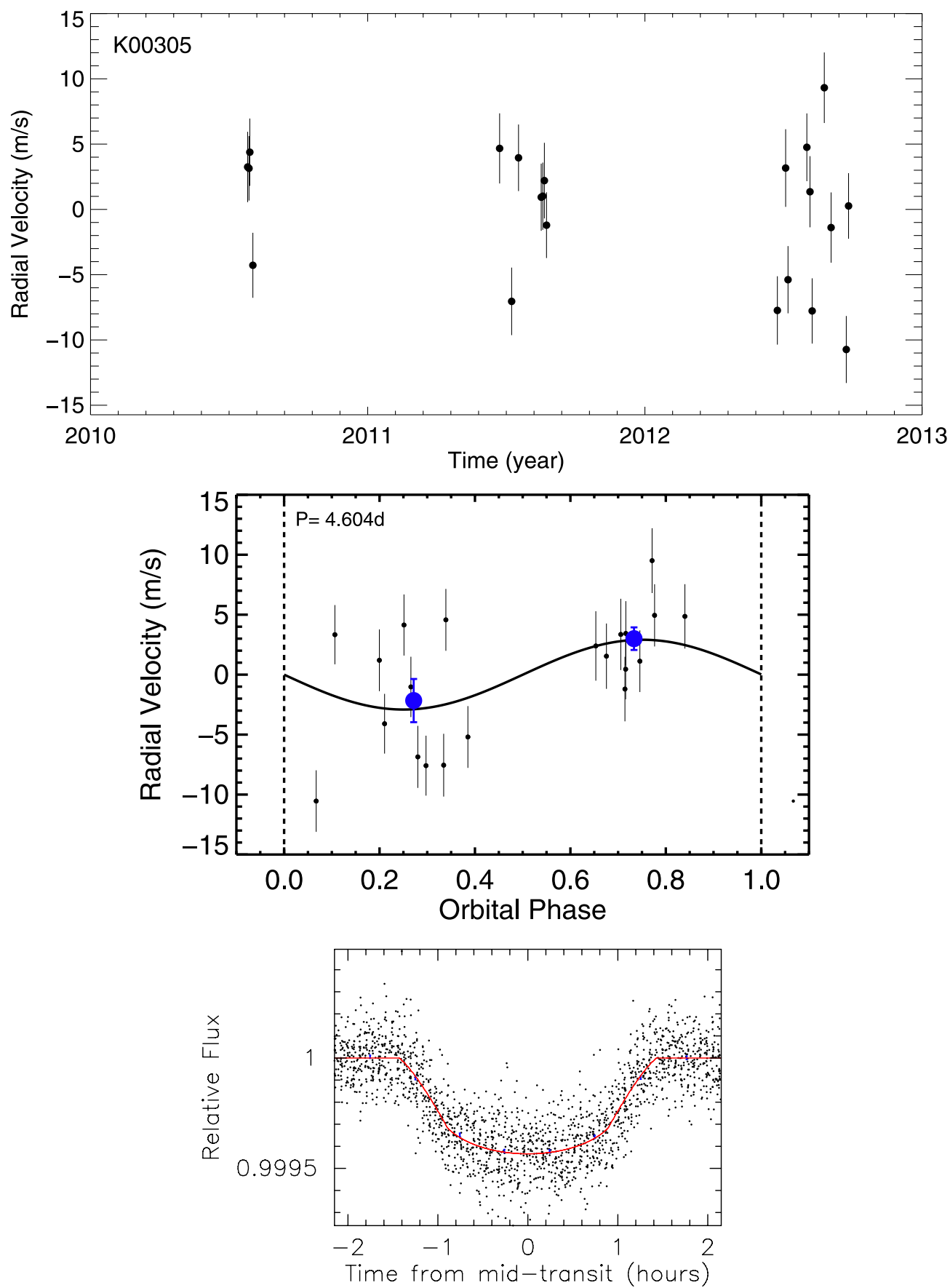

Figure 36. Top: RV vs. time for Kepler-99 (KOI-305). Middle: phase-folded radial velocities for each planet. Bottom: phase-folded Kepler photometry for the planet. The blue points represent binned RVs near quadrature, same as Figure 2. Kepler-99b: $\mathrm{Rp}=1.48 \pm 0.08 R_{\oplus}, \mathrm{Mp}=6.15 \pm 1.3 M_{\oplus}$.

(A color version of this figure is available in the online journal.)

required larger planet (with lower occurrence) and the dilution from the primary star (less detectable) make it less likely that the observed dimming is actually from the secondary star. Thus the probability that a given planet candidate is actually orbiting a secondary star is probably under $25 \%$. Thus, less than $25 \%$ of the planets reported here actually orbit a bound companion star. In such cases the planet is likely to be larger than given here in Table 2.

\subsection{False-positive Probabilities Above 1\%}

The one KOI with a formal FPP above 1\%, KOI-1612.01, merits more detailed attention.
KOI-1612.01 exhibits a $V$-shaped transit light curve, yielding a nominal FPP of $2.1 \%$ (3) stemming primarily from a background eclipsing binary scenario. With its exclusion radius of 2 .' 1 , this planet candidate must remain a candidate, as the background eclipsing binary scenario remains viable with a probability of $2 \%$. The FPP above 1\% excludes KOI-1612.01 from receiving a Kepler number.

The high FPP for KOI-116.01 stems from its transit duration of only $1.4 \mathrm{hr}$, even though the orbital period is 2.4 days orbit around a star of radius $1.23 R_{\odot}$. This short observed transit duration is shorter than expected for such a transit and star, unless the impact parameter is near unity, which is unlikely. 

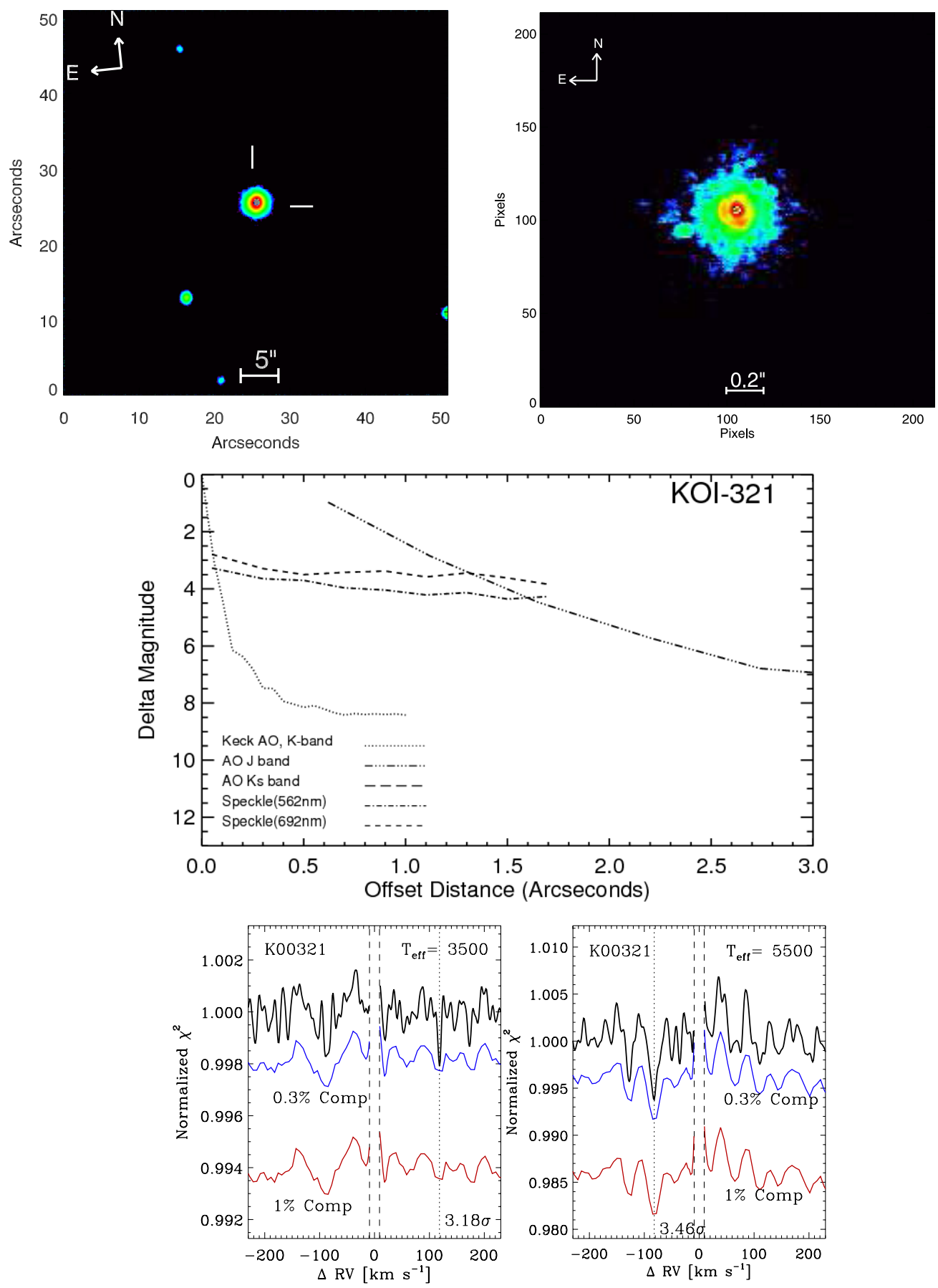

Figure 37. Upper left: seeing-limited image for Kepler-406 (KOI-321). Upper right: adaptive optics image. Middle: limiting magnitudes of companion stars found with Speckle and AO imaging. Lower left and lower right: same as Figure 1, but for Kepler-406. No companions are detected. Any companion brighter than $0.5 \%$ the brightness ( $V$ band) of the primary would have been detected.

(A color version of this figure is available in the online journal.)

The star field is indeed crowded, and the astrometric centroid diagnostics are understandably ambiguous. This light curve of such short duration could instead be caused by a eclipsing binary around a smaller star in the background. Moreover, the transit light curve for KOI-1612.01 is shallow, with a fractional depth of only 0.00003 (see Figure 42, bottom), leaving fractional noise high enough to allow a wide variety of false-positive scenarios.

We note that many systems, such as 108.01 and 108.02, should have FPP reduced by factors of over five due to their occurrence in a system of multiple planets. Such systems apparently have their orbital planes oriented nearly edgeon to our line of sight. Given one transiting planet, such alignment increases the probability of a transit by any other planets in the system (Lissauer et al. 2012, 2013). We do not apply this FPP benefit for multi-transiting-planets here. For a complete assessment of the statistical boost to enable validation of multi-planet transiting systems, with hundreds of planets validated, see Lissauer et al. (2014), and Rowe et al. (2014). 

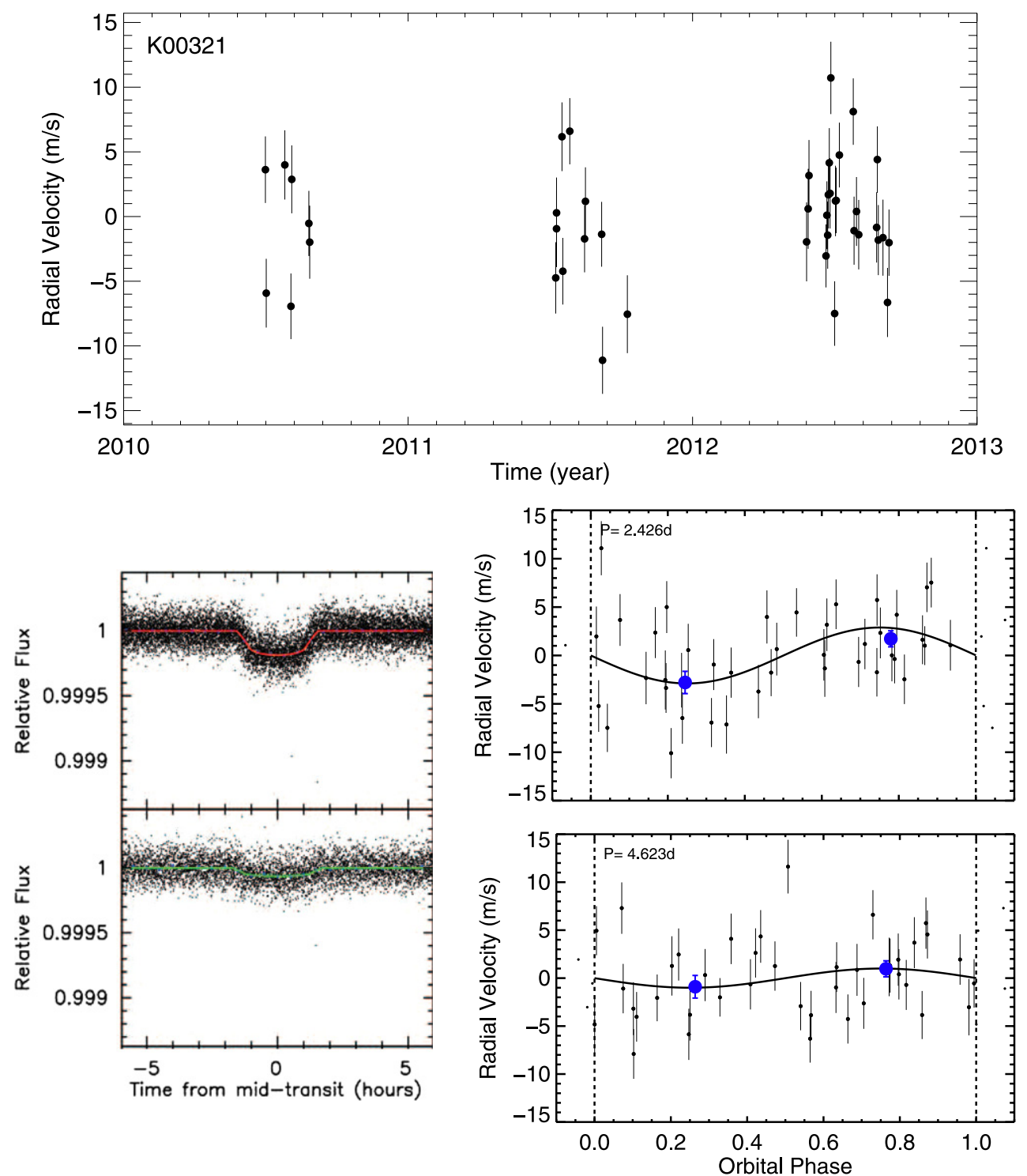

Figure 38. Top: RV vs. time for Kepler-406 (KOI-321). Lower left: phase-folded Kepler photometry for each planet. Lower right: phase-folded radial velocities for each planet. The blue points represent binned RVs near quadrature, same as Figure 2. Kepler-406b: Rp $=1.43 \pm 0.03 R_{\oplus}, \mathrm{Mp}=6.35 \pm 1.4 M_{\oplus}$. Kepler-406 $\mathrm{cp}=$ $0.85 \pm 0.03 R_{\oplus}, \mathrm{Mp}=2.71 \pm 1.8 M_{\oplus}$.

(A color version of this figure is available in the online journal.)

\section{INDIVIDUAL KOIS: OBSERVATIONS, ANALYSIS, AND PLANET PROPERTIES}

We provide here detailed descriptions of the observations made for each KOI individually. We include descriptions of the sequence of follow-up observations organized in chronological order to highlight the reasoning during the reconnaissance work that led to the final measurements with precise RVs and AO made at the Keck 1 and Keck 2 telescopes, respectively. The goal is to highlight the evolution of our understanding of each planet, from preliminary estimates of its radius and orbital period, to the final posterior distributions of the masses, radii, densities, and orbital parameters.

The ground-based follow-up observations were made by the Kepler FOP team from 2009-2012, (Gautier et al. 2010; Batalha et al. 2013), as described in Section 2 with a few additional observations made during the era after the end of the nominal Kepler mission deemed the "Community Follow-up Observing Program" (CFOP). Detailed descriptions of the origin of stellar parameters and planet parameters were previously given in Sections 3 and 5, respectively. ${ }^{48}$

\subsection{KOI-41, Kepler-100}

Three transiting planet candidates were detected in Kepler photometry around Kepler-100 and reported in Borucki et al. (2011) and Batalha et al. (2013). These are now understood to have orbital periods of $6.9,12.8$, and 35.3 days and planet radii of $1.3,2.2$, and $1.6 R_{\oplus}$, respectively. Due to their order of identification, the KOI numbers are not in ascending order of orbital period. A "recon" spectrum (see Section 2.2.1) was taken at the McDonald $2.7 \mathrm{~m}$ telescope in 2009 June, followed by another at the Tillinghast $1.5 \mathrm{~m}$ telescope that same month, and both were analyzed with an early version of the spectroscopic analysis package, "SPC" described by Buchhave et al. (2012) that had $T_{\text {eff }}$ discrimination only accurate to $250 \mathrm{~K}$ and $\log g$ to

\footnotetext{
48 Nearly all of the observations described here are publicly available on the
} CFOP Web site: cfop.ipac.caltech.edu/home/login.php. 

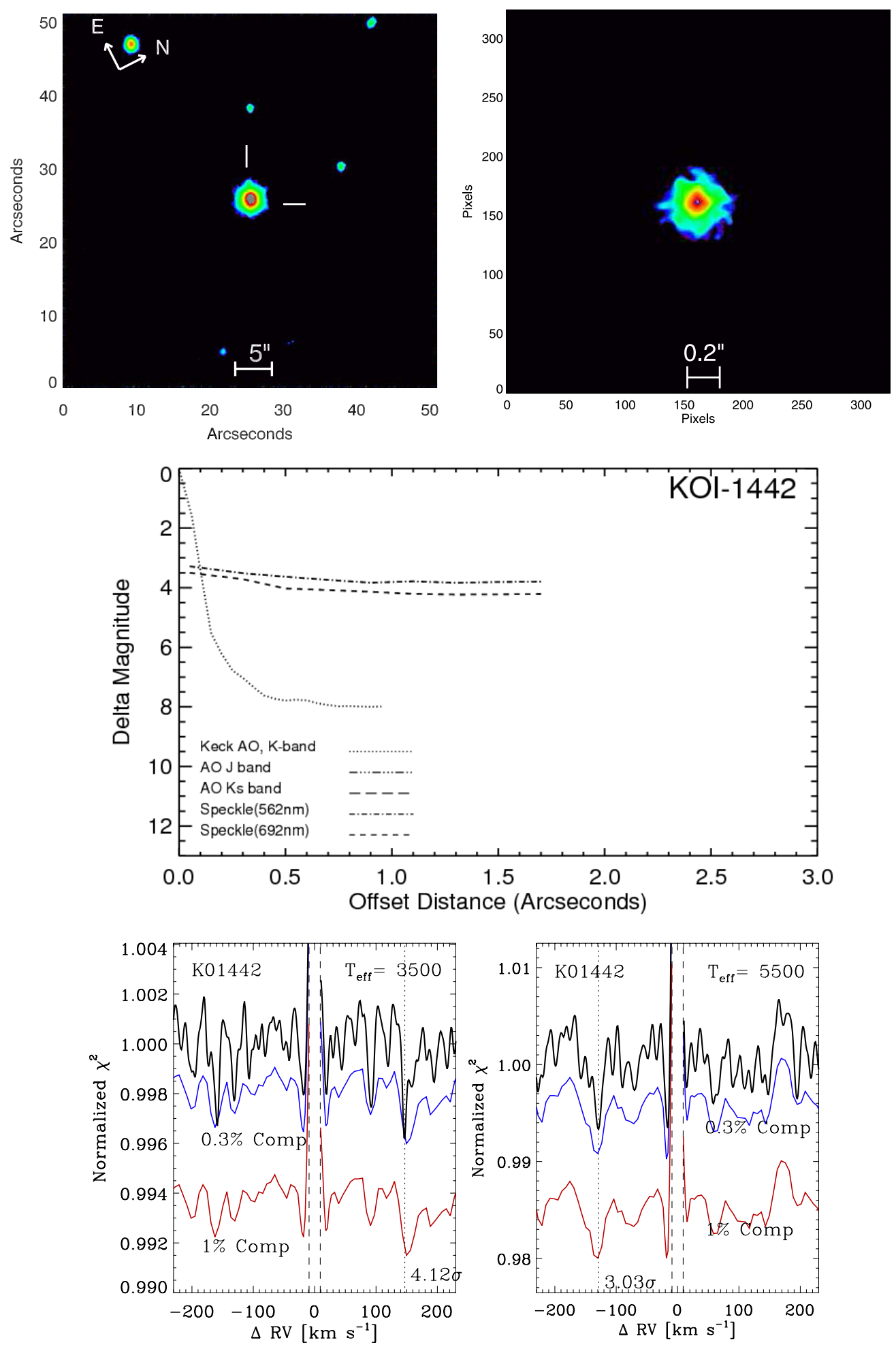

Figure 39. Upper left: seeing-limited image for Kepler-407 (KOI-1442). Upper right: adaptive optics image from Keck NIRC2. Middle: limiting magnitudes of companion stars from Speckle and AO imaging. Lower left and lower right: same as Figure 1, but for Kepler-407. No secondary lines from a companion are detected. Any companion brighter than $0.5 \%$ the brightness ( $V$ band) of the primary would have been detected.

(A color version of this figure is available in the online journal.)

0.2 dex. The resulting values of $T_{\text {eff }}$ and $\log g$ were found to be consistent with those in the KIC. No large RV variations (over $1 \mathrm{~km} \mathrm{~s}^{-1}$ ) were found, and the rotational Doppler broadening, $v \sin i$, was found to be less than $4 \mathrm{~km} \mathrm{~s}^{-1}$. These stellar parameters based on "recon" spectroscopy, along with the relative brightness of Kepler-100, made it a good candidate for high signal-to-noise, high-resolution spectroscopy to better constrain stellar parameters and to carry out precise Doppler measurements.

A "template" spectrum was obtained with Keck-HIRES (no iodine in the beam). Its analysis with SME gave values of $T_{\text {eff }}$ and $[\mathrm{Fe} / \mathrm{H}]$, used to constrain the asteroseismology 

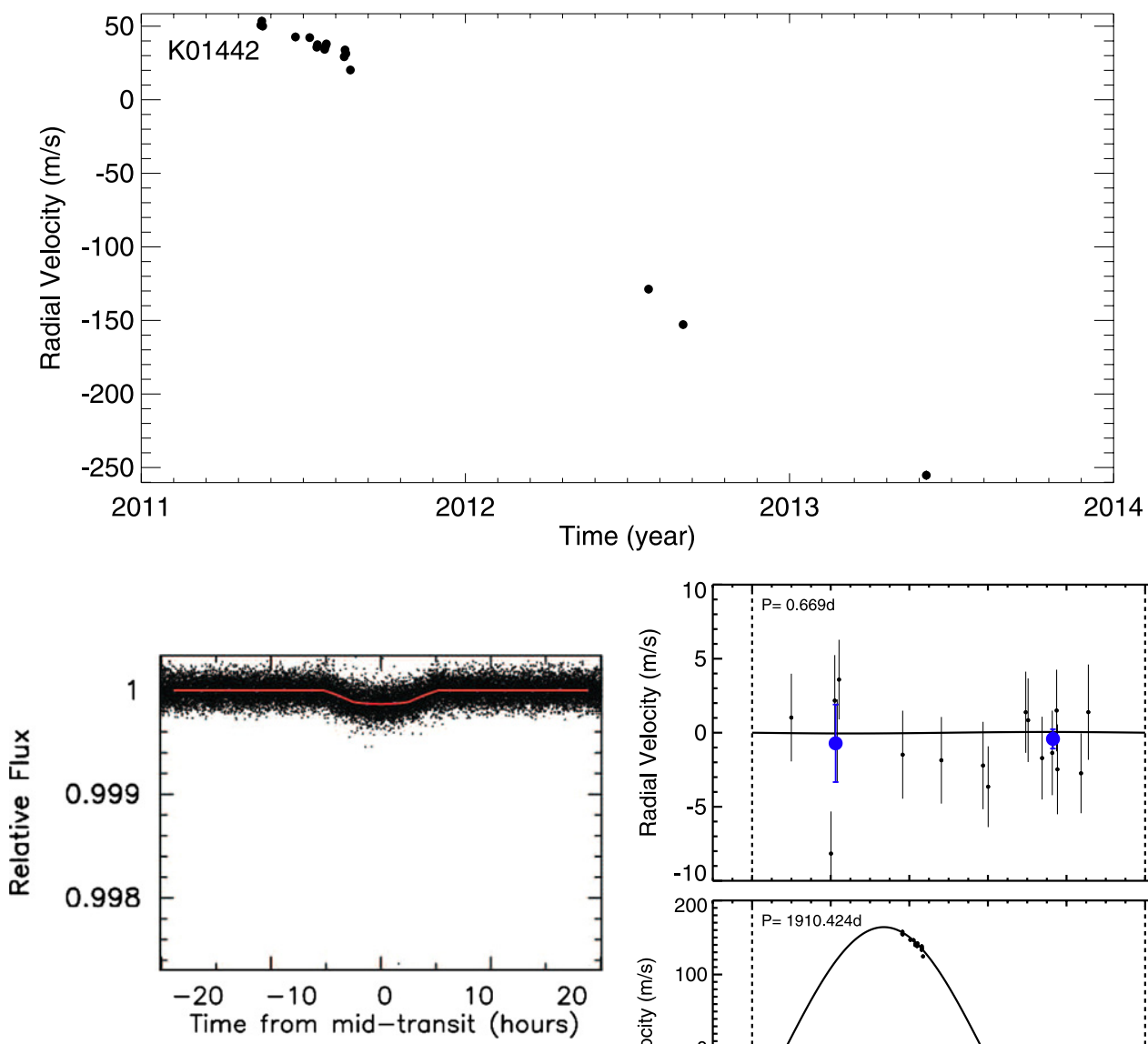

Non-Transiting

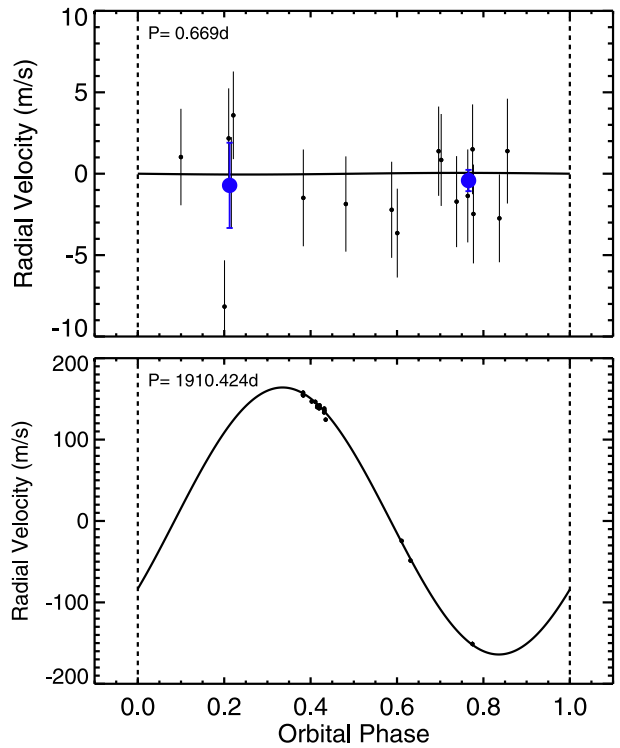

Figure 40. Top: RV vs. time for Kepler-407 (KOI-1442). Lower left: phase-folded Kepler photometry for the transiting planet. Lower right: phase-folded radial velocities for each planet. The blue points represent binned RVs near quadrature, same as Figure 2. Kepler-407b: Rp $=1.07 \pm 0.02 R_{\oplus}, M p=0.06 \pm 1.2 M_{\oplus}$. Kepler-407c: $\mathrm{Rp}=\mathrm{NA}, \mathrm{Mp}=4000 \pm 2000 M_{\oplus}$, period $=3000 \pm 500 \mathrm{~d}$. Kepler-407 could be a planet, brown dwarf, or a star, depending on the inclination of the orbit. (A color version of this figure is available in the online journal.)

analysis, coupled with stellar interiors modeling, to yield stellar mass and radius (described in detail in Section 3). A subsequent iteration between SME and asteroseismology brought a quick convergence of stellar mass and radius. The final stellar parameters are $T_{\text {eff }}=5825 \pm 75 \mathrm{~K}, \log g=4.13 \pm$ $0.03, v \sin i=3.7 \pm 1.0 \mathrm{~km} \mathrm{~s}^{-1}$ and $[\mathrm{Fe} / \mathrm{H}]=0.02 \pm 0.10$, (see Table 1).

High-resolution imaging was acquired in 2010 May with the ARIES AO system on the MMT, under seeing of $0^{\prime \prime} 1$ in the $K s$ band and $0^{\prime \prime} .2$ in the $J$ band. Speckle imaging was taken at the WIYN telescope in 2010 June. (citations for all instruments are given at the beginning of Section 2.2.) Neither imaging technique found any nearby companion stars within 6 " of the primary.

Keck AO imaging was performed on 2013 June 13/14 and $14 / 15$, using a Bracket-gamma filter to avoid saturation, as we had to use for all stars brighter than $K p=11 \mathrm{mag}$. The images of Kepler-100 have a PSF with a FWHM of 0'.05 (described in detail in Section 2.2.3). A full detectability curve at $K^{\prime}$ band $(2.2 \mu)$, giving the detection threshold of any neighboring stars as a function of angular separation, is shown in Figure 1. No companion was seen.
Figure 1 shows a seeing-limited image with the spectrometer slit causing the vertical line (upper left panel), an AO image(upper right), the detection threshold in delta magnitudes achieved with each imaging method (middle panel), and the limits on companions as determined by searching this star's HIRES spectrum for secondary lines (lower panel).

The low rotational line broadening $(v \sin i)$ and the lack of nearby stellar companions made Kepler-100 a high quality target for precise RV measurements with Keck-HIRES. They began 2009 July 29 and span 1221 days (Figure 2, top panel), including 44 precise RVs. The RVs show a weak correlation with the ephemeris of the 6.9 days transiting planet identified by Kepler, limiting the planet mass to be less than $9.6 M_{\oplus}$ at the $1 \sigma$ level. This upper limit to the planet mass corresponds to a bulk density of the planet of $20.5 \mathrm{~g} \mathrm{~cm}^{-3}$. Since this density is greater than any plausible solid material, taking into account gravitational compression, the limit is not physically meaningful. Additional RV observations would likely push this limiting mass lower.

The RVs also do not correlate with the ephemeris of the 12.8 days transiting planet from Kepler photometry, but the upper limits to the planet mass are physically meaningful. The $1 \sigma$ upper limit to the mass is $4.8 M_{\oplus}$, corresponding to a bulk 

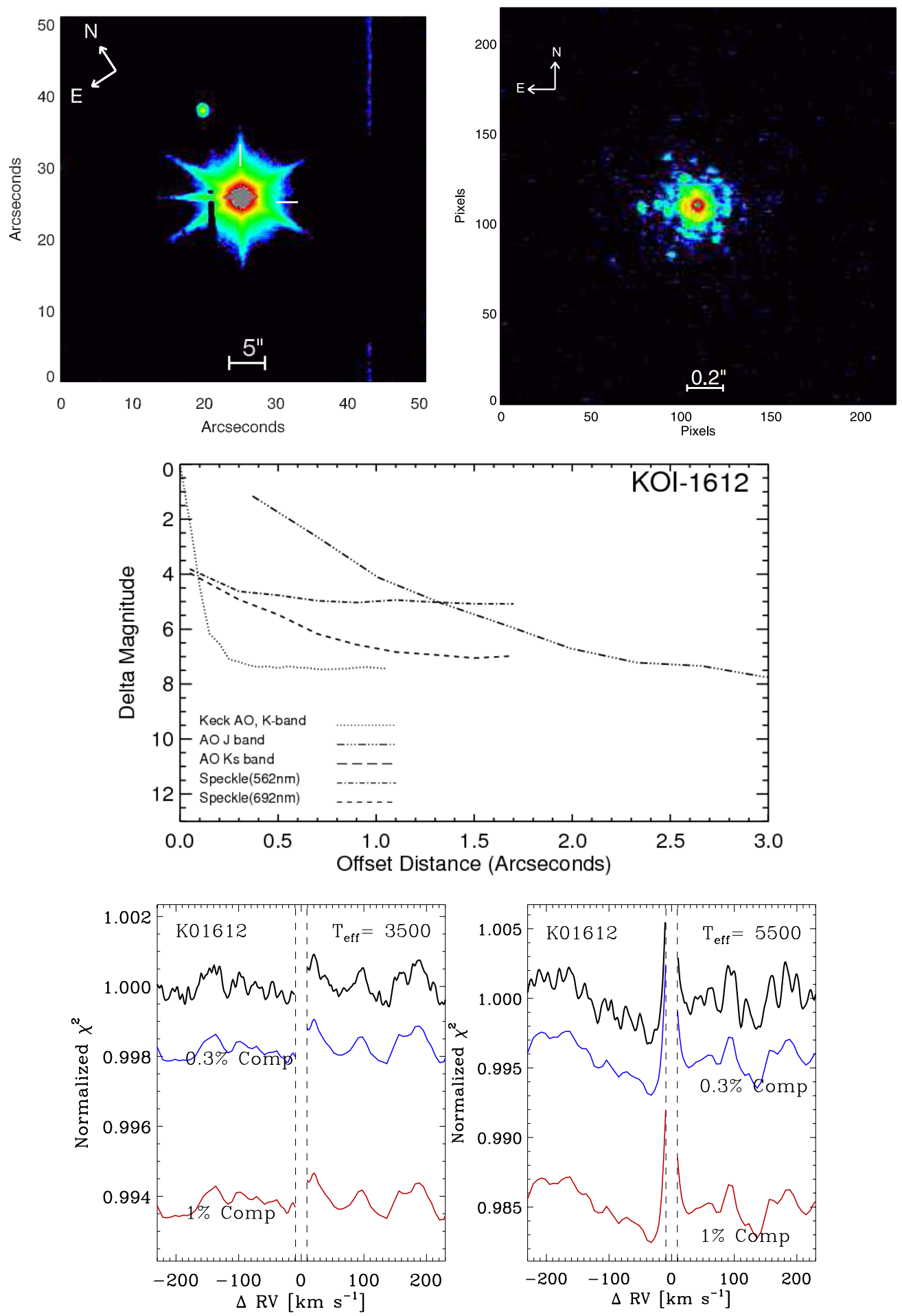

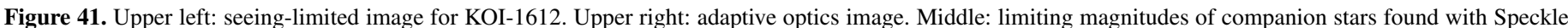

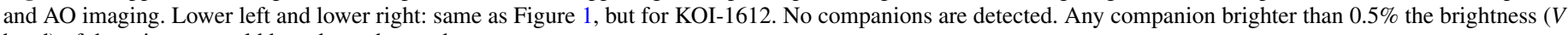
band) of the primary would have been detected.

(A color version of this figure is available in the online journal.)

density of $2.0 \mathrm{~g} \mathrm{~cm}^{-3}$. If this planet had consisted of mostly dense material, such as iron with an expected density near $10 \mathrm{~g} \mathrm{~cm}^{-3}$, the RV amplitude would have been $5 \times$ larger, making such an RV signal easily detectable. But such was not the case. Instead, this planet must consist of a significant admixture of lighter materials, consistent with the trend described in Section 8 in which planets with radii greater than $2.0 R_{\oplus}$ typically have densities less than $3.0 \mathrm{~g} \mathrm{~cm}^{-3}$. The RVs also do not correlate with the 35 day planet ephemeris. The peak value in the posterior distribution of the planet mass is below zero, meaning that the measured mass is consistent with zero. The $1 \sigma$ upper limit for the planet mass is $1.7 M_{\oplus}$ and $1.9 \mathrm{~g} \mathrm{~cm}^{-3}$. Figure 2 (bottom right panel) shows the phased RVs for each planet. 

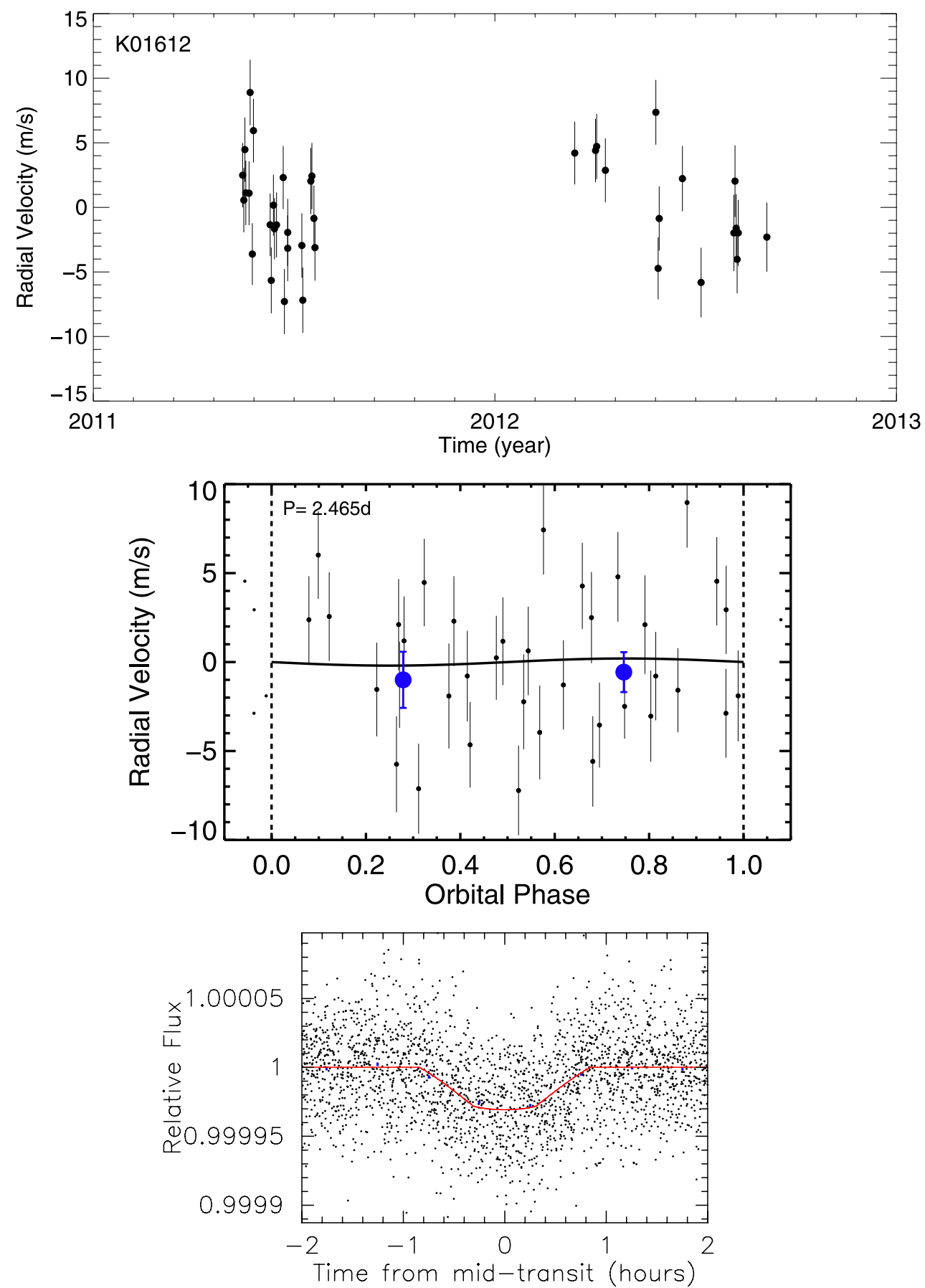

Figure 42. Top: RV vs. time for KOI-1612. Middle: phase-folded radial velocities for each planet. Bottom: phase-folded Kepler photometry for the planet. The blue points represent binned RVs near quadrature, same as Figure 2. KOI-1612.01: $\mathrm{Rp}=0.82 \pm 0.03 R_{\oplus}, \mathrm{Mp}=0.48 \pm 3.2 M_{\oplus}$.

(A color version of this figure is available in the online journal.)

The full set of RVs are plotted in Figure 2 (top) and listed in Table 4, along with chromospheric $\log R_{\mathrm{HK}}^{\prime}$ values measured from the same spectra as the RVs, making them simultaneously obtained.

\subsection{KOI-69, Kepler-93}

Kepler photometry identified Kepler-93 as having one transiting planet candidate with a period of 4.7 days and a radius of $1.5 R_{\oplus}$. A second orbiting companion is indicated (as described below) by the precise RVs that exhibit a linear trend of $+11 \mathrm{~m} \mathrm{~s}^{-1} \mathrm{yr}^{-1}$. During the past $4 \mathrm{yr}$, the RVs show no departure from a straight line at the $1 \mathrm{~m} \mathrm{~s}^{-1}$ level.
Follow-up recon spectroscopy commenced in 2009 August at McDonald $2.7 \mathrm{~m}$ and the $2.6 \mathrm{~m}$ Nordic Optical telescope. Moderate signal-to-noise spectra were acquired, yielding spectral parameters found with SPC analysis that were consistent with the values in the KIC. The $v \sin i$ was measured to be $<2 \mathrm{~km} \mathrm{~s}^{-1}$, consistent with a slowly rotating main sequence star G5V, and no RV variation was seen between the two spectra at the $500 \mathrm{~m} \mathrm{~s}^{-1}$ level (confirmed by later precise RVs). SME analysis on the Keck-HIRES template spectrum showed the star to be slightly cooler than the KIC and recon-determined parameters. Final stellar parameters including stellar mass and radius were calculated using asteroseismology with SME values 

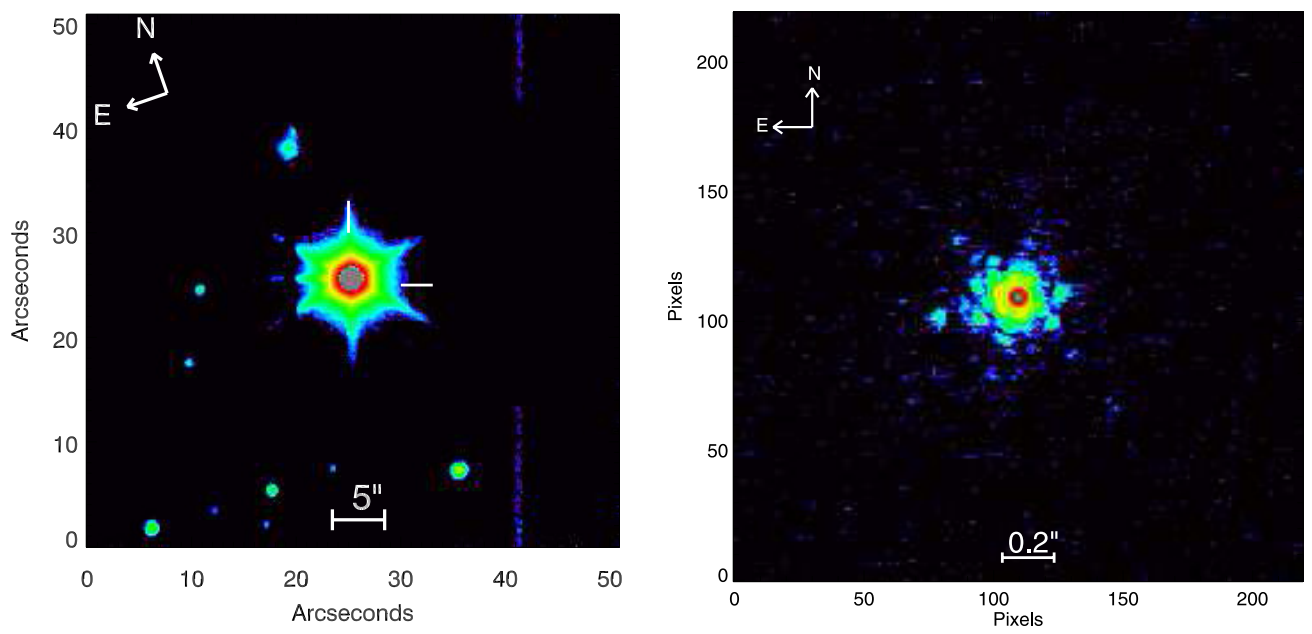

K01925 Kprime
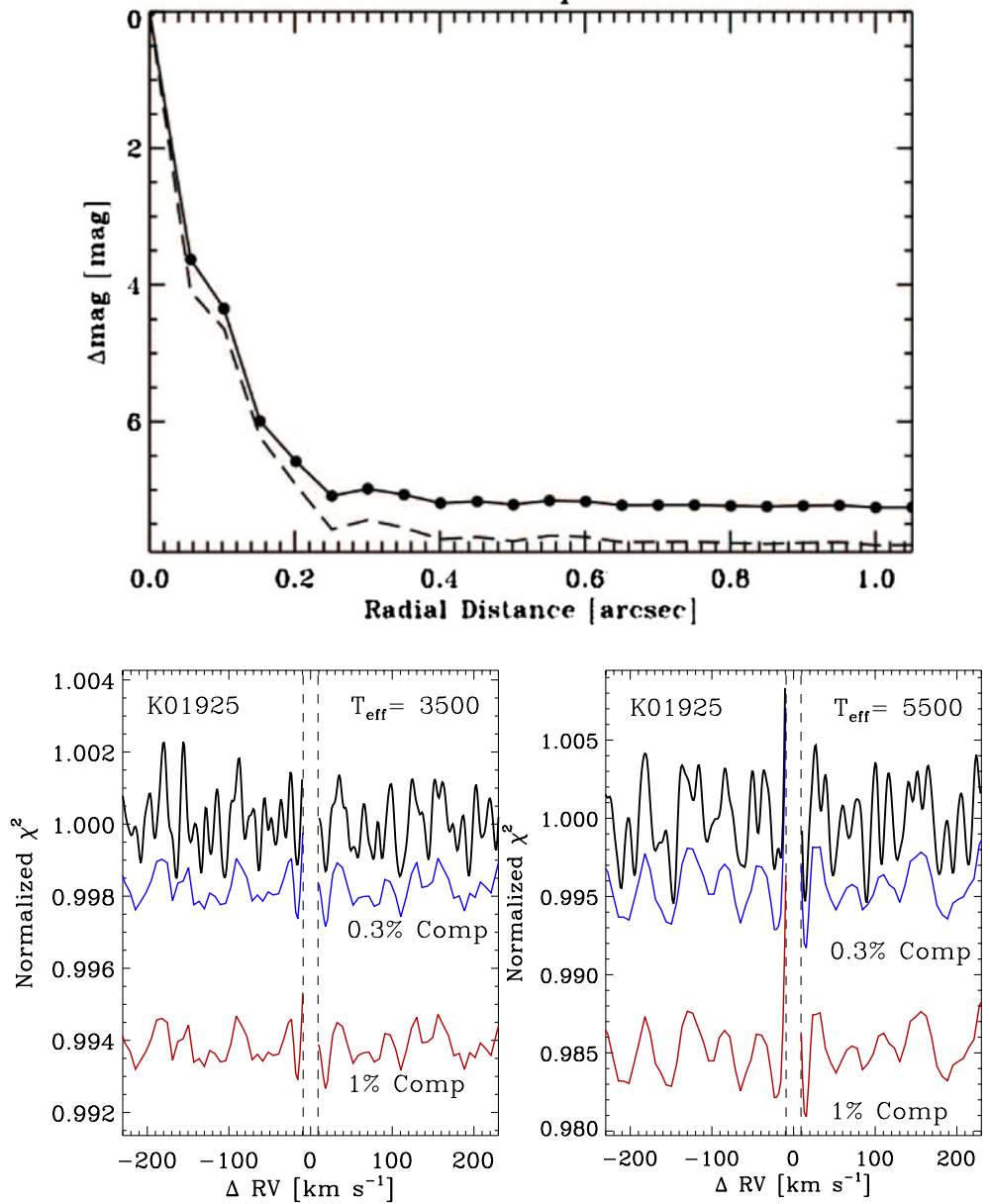

Figure 43. Upper left: seeing-limited image for Kepler-409b (KOI-1925). Upper right: adaptive optics image. Middle: limiting magnitudes of companion stars found with Speckle and AO imaging. Lower left and lower right: same as Figure 1, but for Kepler-409. No companions are detected. Any companion brighter than 0.5\% the brightness ( $V$ band) of the primary would have been detected.

(A color version of this figure is available in the online journal.)

used as input parameters, along with the usual iteration between the two. The final stellar parameters are $T_{\text {eff }}=5669 \pm 75 \mathrm{~K}$, $\log g=4.47 \pm 0.03$ and $[\mathrm{Fe} / \mathrm{H}]=0.02 \pm 0.10$ (see Section 3$)$. Table 1 lists the final stellar parameters including stellar mass and radius.

Speckle imaging was first obtained in 2009 October and a binary companion was suggested 0.05 away with a delta magnitude of 1.4 mag. Two additional speckle images were taken and the secondary star was not detected. A warning about the possible existence of this companion appears in Table 3 of Borucki et al. (2011) but it should be disregarded. AO imaging in 2010 May at MMT-ARIES confirmed no secondary stars were present from $1^{\prime \prime}-6^{\prime \prime}$ of the primary, but the putative 0.05 companion would have fallen within the inner working angle of 

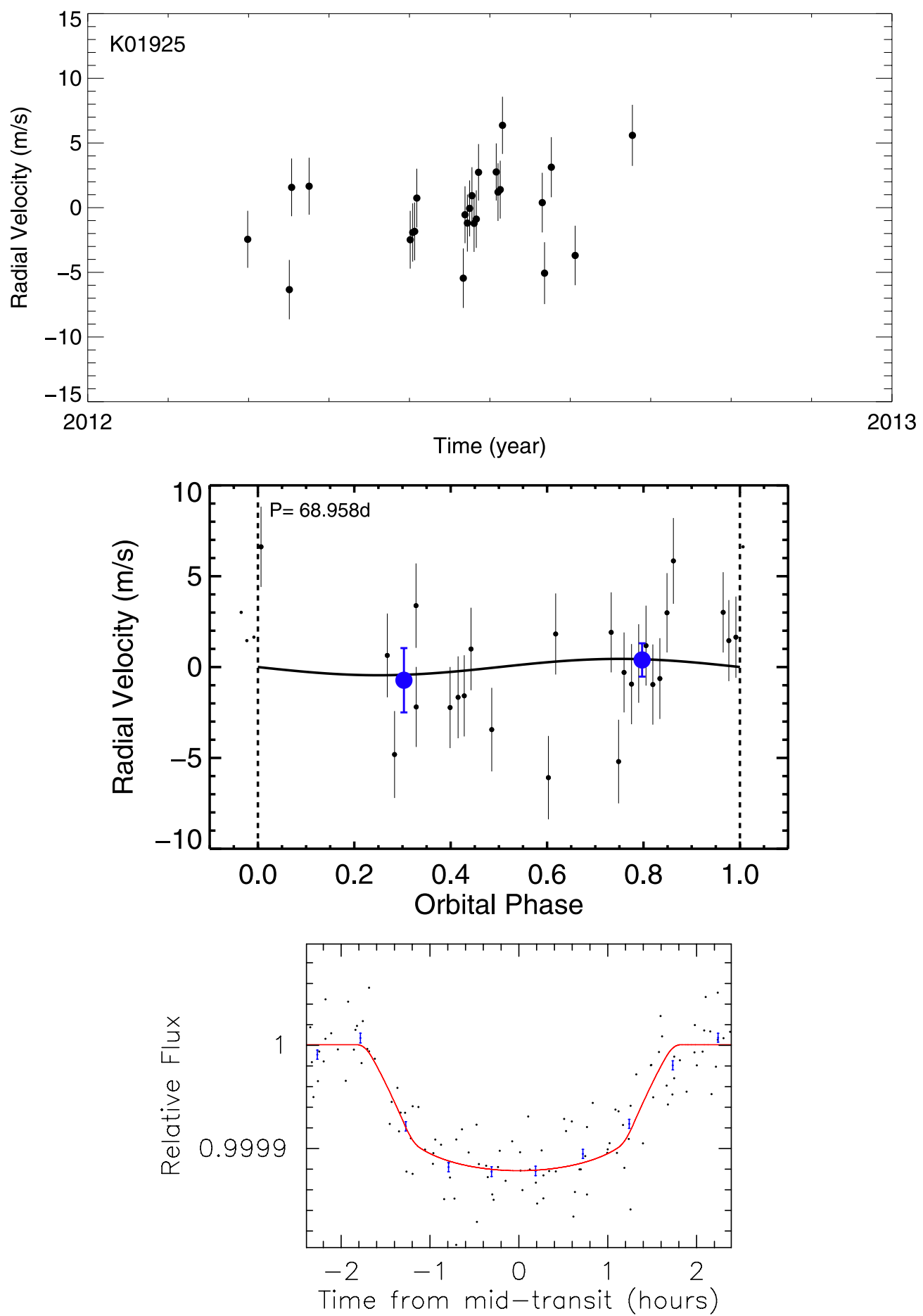

Figure 44. Top: RV vs. time for Kepler-409 (KOI-1925). Middle: phase-folded radial velocities for each planet. Bottom: phase-folded Kepler photometry for each planet. The blue points represent binned RVs near quadrature, same as Figure 2. Kepler-409b: Rp $=1.19 \pm 0.03 R_{\oplus}, \mathrm{Mp}=2.69 \pm 6.2 M_{\oplus}$.

(A color version of this figure is available in the online journal.)

ARIES. The field of view near this KOI is shown in an image from the Keck-HIRES guide camera, which is a seeing limited and shown in Figure 3 (top left). Keck AO imaging on 2013 June 13/14 and 14/15 yielded a FWHM of 0'.05 Figure 3 (top right), with a full detectability curve given in Figure 3 (middle). No companion was seen. Limits on companions from the highresolution imaging are displayed in Figure 3 (bottom).

Keck-HIRES precise RVs span 1132 days, from 2009 July to 2012 September (Figure 4, top). The most prominent feature in the RVs is the linear trend of $11.2 \pm 1.5 \mathrm{~m} \mathrm{~s}^{-1} \mathrm{yr}^{-1}$. Since we see no curvature, the orbital period of the companion causing the linear trend is much longer than the time baseline of nearly $4 \mathrm{yr}$. Given the line-of-sight component of acceleration during $4 \mathrm{yr}$, we can place lower limits on the mass and period $\left(M>3 M_{\mathrm{JUP}}\right.$ and $P>5 \mathrm{yr}$ ), based on the duration and magnitude of the $\mathrm{RV}$ trend. The linear RV trend suggests the period is much longer than $10 \mathrm{yr}$, indicating that the orbiting companion has a mass at least tens of Jupiter masses. It could be an M dwarf or brown dwarf orbiting beyond $5 \mathrm{au}$, or perhaps a compact stellar object, all being too dark to be revealed in the Keck-AO 
Table 1

\begin{tabular}{|c|c|c|c|c|c|c|c|c|c|c|c|c|c|}
\hline Kepler No. & KOI & KIC & R.A. & Decl. & $\begin{array}{l}T_{\text {eff }} \\
(\mathrm{K})\end{array}$ & $\begin{array}{c}\text { Stellar } \\
\log g\end{array}$ & {$[\mathrm{Fe} / \mathrm{H}]$} & $\begin{array}{c}M_{*} \\
\left(M_{\odot}\right)\end{array}$ & $\begin{array}{c}R_{*} \\
\left(R_{\odot}\right)\end{array}$ & $\begin{array}{c}V \sin i \\
\left(\mathrm{~km} \mathrm{~s}^{-1}\right)\end{array}$ & $\begin{array}{l}\text { Kepler } \\
\text { Magn. }\end{array}$ & $\begin{array}{l}\text { Age } \\
\text { (Gyr) }\end{array}$ & $\begin{array}{c}\text { Source }^{\mathrm{a}} \\
\text { MCMC+ }\end{array}$ \\
\hline Kepler-100 & 41 & 6521045 & $19: 25: 32.6$ & $41: 59: 24$ & $5825 \pm 75$ & $4.125 \pm 0.03$ & $+0.02 \pm 0.10$ & $1.08 \pm 0.06$ & $1.49 \pm 0.04$ & 3.7 & 11.20 & 6.46 & AS \\
\hline Kepler-93 & 69 & 3544595 & $19: 25: 40.3$ & $38: 40: 20$ & $5669 \pm 75$ & $4.468 \pm 0.03$ & $-0.18 \pm 0.10$ & $0.91 \pm 0.06$ & $0.92 \pm 0.02$ & 0.5 & 9.93 & 5.05 & AS \\
\hline Kepler-102 & 82 & 10187017 & $18: 45: 55.8$ & $47: 12: 28$ & $4903 \pm 74$ & $4.607 \pm 0.03$ & $+0.08 \pm 0.07$ & $0.80 \pm 0.06$ & $0.74 \pm 0.02$ & 0.5 & 11.49 & 1.41 & SME \\
\hline Kepler-94 & 104 & 10318874 & $18: 44: 46.7$ & $47: 29: 49$ & $4781 \pm 98$ & $4.590 \pm 0.04$ & $+0.34 \pm 0.07$ & $0.81 \pm 0.06$ & $0.76 \pm 0.03$ & 0.5 & 12.90 & 1.41 & SME \\
\hline Kepler-103 & 108 & 4914423 & $19: 15: 56.2$ & 40:03:52 & $5845 \pm 88$ & $4.162 \pm 0.04$ & $+0.07 \pm 0.11$ & $1.09 \pm 0.07$ & $1.44 \pm 0.04$ & 2.5 & 12.29 & 5.70 & AS \\
\hline Kepler-106 & 116 & 8395660 & $20: 03: 27.3$ & $44: 20: 15$ & $5858 \pm 114$ & $4.407 \pm 0.14$ & $-0.12 \pm 0.11$ & $1.00 \pm 0.06$ & $1.04 \pm 0.17$ & 0.3 & 12.88 & 4.83 & SME \\
\hline Kepler-95 & 122 & 8349582 & $18: 57: 55.7$ & $44: 23: 52$ & $5699 \pm 74$ & $4.171 \pm 0.04$ & $+0.30 \pm 0.10$ & $1.08 \pm 0.08$ & $1.41 \pm 0.04$ & 0.7 & 12.35 & 5.63 & AS \\
\hline Kepler-109 & 123 & 5094751 & $19: 21: 34.2$ & 40:17:05 & $5952 \pm 75$ & $4.211 \pm 0.04$ & $-0.08 \pm 0.10$ & $1.04 \pm 0.06$ & $1.32 \pm 0.04$ & 1.0 & 12.36 & 5.73 & AS \\
\hline Kepler-48 & 148 & 5735762 & $19: 56: 33.4$ & 40:56:56 & $5194 \pm 73$ & $4.487 \pm 0.05$ & $+0.17 \pm 0.07$ & $0.88 \pm 0.06$ & $0.89 \pm 0.05$ & 0.5 & 13.04 & 3.14 & SME \\
\hline Kepler-113 & 153 & 12252424 & 19:11:59.4 & $50: 56: 39$ & $4725 \pm 74$ & $4.636 \pm 0.03$ & $+0.05 \pm 0.07$ & $0.75 \pm 0.06$ & $0.69 \pm 0.02$ & 0.4 & 13.46 & 6.89 & SME \\
\hline Kepler-25 & 244 & 4349452 & 19:06:33.2 & $39: 29: 16$ & $6270 \pm 79$ & $4.278 \pm 0.03$ & $-0.04 \pm 0.10$ & $1.19 \pm 0.06$ & $1.31 \pm 0.02$ & 9.5 & 10.73 & 11.00 & AS \\
\hline Kepler-37 & 245 & 8478994 & $18: 56: 14.2$ & 44:31:05 & $5417 \pm 75$ & $4.567 \pm 0.05$ & $-0.32 \pm 0.07$ & $0.80 \pm 0.07$ & $0.77 \pm 0.03$ & 0.5 & 9.70 & 5.66 & AS \\
\hline Kepler-68 & 246 & 11295426 & $19: 24: 07.7$ & 49:02:24 & $5793 \pm 74$ & $4.282 \pm 0.02$ & $+0.12 \pm 0.07$ & $1.08 \pm 0.05$ & $1.24 \pm 0.02$ & 0.5 & 10.00 & 6.30 & AS \\
\hline Kepler-96 & 261 & 5383248 & 19:48:16.7 & $40: 31: 30$ & $5690 \pm 73$ & $4.421 \pm 0.08$ & $+0.04 \pm 0.07$ & $1.00 \pm 0.06$ & $1.02 \pm 0.09$ & 0.5 & 10.30 & 2.34 & SME \\
\hline Kepler-131 & 283 & 5695396 & 19:14:07.4 & $40: 56: 32$ & $5685 \pm 74$ & $4.417 \pm 0.08$ & $+0.12 \pm 0.07$ & $1.02 \pm 0.06$ & $1.03 \pm 0.10$ & 0.4 & 11.52 & 3.66 & SME \\
\hline Kepler-97 & 292 & 11075737 & 19:09:18.3 & $48: 40: 24$ & $5779 \pm 74$ & $4.430 \pm 0.08$ & $-0.20 \pm 0.07$ & $0.94 \pm 0.06$ & $0.98 \pm 0.09$ & 0.5 & 12.87 & 8.42 & SME \\
\hline Kepler-98 & 299 & 2692377 & $19: 02: 38.8$ & $37: 57: 52$ & $5539 \pm 73$ & $4.341 \pm 0.10$ & $+0.18 \pm 0.07$ & $0.99 \pm 0.06$ & $1.11 \pm 0.12$ & 0.5 & 12.90 & 2.79 & SME \\
\hline Kepler-99 & 305 & 6063220 & $19: 49: 24.9$ & 41:18:00 & $4782 \pm 129$ & $4.605 \pm 0.05$ & $+0.18 \pm 0.07$ & $0.79 \pm 0.06$ & $0.73 \pm 0.04$ & 0.5 & 12.97 & 1.47 & SME \\
\hline Kepler-406 & 321 & 8753657 & $19: 27: 23.5$ & 44:58:05 & $5538 \pm 75$ & $4.409 \pm 0.02$ & $+0.18 \pm 0.07$ & $1.07 \pm 0.06$ & $1.07 \pm 0.02$ & 0.4 & 12.52 & 5.84 & AS \\
\hline Kepler-407 & 1442 & 11600889 & 19:04:08.7 & 49:36:52 & $5476 \pm 75$ & $4.426 \pm 0.06$ & $+0.33 \pm 0.07$ & $1.00 \pm 0.06$ & $1.01 \pm 0.07$ & 2.0 & 12.52 & 7.47 & SME \\
\hline$\ldots$ & 1612 & 10963065 & 18:59:08.6 & $48: 25: 23$ & $6104 \pm 74$ & $4.294 \pm 0.03$ & $-0.20 \pm 0.10$ & $1.08 \pm 0.07$ & $1.23 \pm 0.03$ & 3.1 & 8.77 & 6.68 & AS \\
\hline Kepler-409 & 1925 & 9955598 & $19: 34: 43.0$ & 46:51:09 & $5460 \pm 75$ & $4.499 \pm 0.03$ & $+0.08 \pm 0.10$ & $0.92 \pm 0.06$ & $0.89 \pm 0.02$ & 2.0 & 9.44 & 6.80 & AS \\
\hline
\end{tabular}

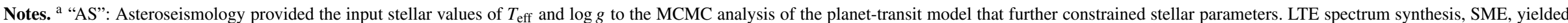

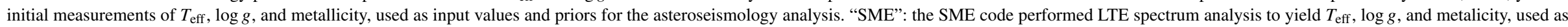
input to the MCMC analysis of the planet transit model. Iteration with Yonsei-Yale stellar models constrained all stellar values. 
Table 2

Planet Properties and Orbital Parameters

\begin{tabular}{|c|c|c|c|c|c|c|c|c|c|c|c|c|}
\hline Kepler No. & KOI & $\begin{array}{c}\text { Period }^{\mathrm{a}} \\
\text { (days) }\end{array}$ & $\begin{array}{c}\text { Radius }^{\mathrm{b}} \\
\left(R_{\oplus}\right)\end{array}$ & $\begin{array}{l}\operatorname{Mass}(\text { peak })^{\mathrm{c}} \\
\quad\left(M_{\oplus}\right)\end{array}$ & $\begin{array}{c}\operatorname{Mass}^{\mathrm{d}} \\
95 \%\left(M_{\oplus}\right)\end{array}$ & $\begin{array}{l}\text { Planet Density } \\
\qquad\left(\mathrm{g} \mathrm{cm}^{-3}\right)\end{array}$ & $\begin{array}{c}K^{\mathrm{f}} \\
\left(\mathrm{m} \mathrm{s}^{-1}\right)\end{array}$ & $\begin{array}{l}\text { Stellar Density } \\
\quad\left(\mathrm{g} \mathrm{cm}^{-3}\right)\end{array}$ & $\begin{array}{c}\text { Impact } \\
\text { Param. }(b)\end{array}$ & $R_{\mathrm{P}} / R_{*}$ & $\begin{array}{c}\text { Midtransit } E \\
\text { (BJD-2454900) }\end{array}$ & Reduced Chi \\
\hline Kepler-100c & 41.01 & 12.8159 & $2.20 \pm 0.05$ & $0.85 \pm 4.0$ & 7.05 & $0.35 \pm 1.65$ & $0.18 \pm 0.8$ & $0.44 \pm 0.01$ & $0.05 \pm 0.04$ & 0.013550 & 55.94713 & 1.185 \\
\hline Kepler-100b & 41.02 & 6.88705 & $1.32 \pm 0.04$ & $7.34 \pm 3.2$ & 10.0 & $\mathbf{1 4 . 2 5} \pm \mathbf{6 . 3 3}$ & $1.90 \pm 0.8$ & $0.44 \pm 0.01$ & $0.54 \pm 0.02$ & 0.008094 & 66.17797 & 1.110 \\
\hline Kepler-100d & 41.03 & 35.3331 & $1.61 \pm 0.05$ & $-4.36 \pm 4.1$ & 3.0 & $-5.72 \pm 6.00$ & $-0.79 \pm 1.0$ & $0.44 \pm 0.01$ & $0.75 \pm 0.01$ & 0.009926 & 86.98031 & 1.261 \\
\hline Kepler-93b & 69.01 & 4.72674 & $1.50 \pm 0.03$ & $2.59 \pm 2.0$ & 6.1 & $4.17 \pm 3.29$ & $1.05 \pm 0.8$ & $1.64 \pm 0.01$ & $0.20 \pm 0.02$ & 0.014927 & 67.92580 & 1.017 \\
\hline Kepler-93c & $69.10^{\mathrm{g}}$ & $>1460$ & $\ldots$ & $>954$ & $\ldots$ & $\ldots$ & $>60$ & $\ldots$ & $\ldots$ & $\ldots$ & $\ldots$ & $\ldots$ \\
\hline Kepler-102e & 82.01 & 16.1457 & $2.22 \pm 0.07$ & $8.93 \pm 2.0$ & 11.7 & $4.68 \pm 1.12$ & $2.77 \pm 0.6$ & $2.76 \pm 0.09$ & $0.30 \pm 0.03$ & 0.027639 & 67.75384 & 1.026 \\
\hline Kepler-102d & 82.02 & 10.3117 & $1.18 \pm 0.04$ & $3.80 \pm 1.8$ & 6.6 & $13.27 \pm 6.46$ & $1.37 \pm 0.6$ & $2.76 \pm 0.09$ & $0.22 \pm 0.05$ & 0.014682 & 67.07920 & 0.950 \\
\hline Kepler-102f & 82.03 & 27.4536 & $0.88 \pm 0.03$ & $0.62 \pm 3.3$ & 5.2 & $4.92 \pm 24.5$ & $2.11 \pm 0.8$ & $2.76 \pm 0.09$ & $0.57 \pm 0.03$ & 0.010963 & 78.02565 & 1.404 \\
\hline Kepler-102c & 82.04 & 7.07142 & $0.58 \pm 0.02$ & $-1.58 \pm 2.0$ & 3.0 & $-44.24 \pm 56.7$ & $-1.00 \pm 0.6$ & $2.76 \pm 0.09$ & $0.06 \pm 0.05$ & 0.007228 & 72.98486 & 0.883 \\
\hline Kepler-102b & 82.05 & 5.28696 & $0.47 \pm 0.02$ & $0.41 \pm 1.6$ & 4.3 & $23.29 \pm 94.93$ & $0.19 \pm 0.7$ & $2.76 \pm 0.09$ & $0.06 \pm 0.06$ & 0.005800 & 68.84920 & 0.958 \\
\hline Kepler-94b & 104.01 & 2.50806 & $3.51 \pm 0.15$ & $10.84 \pm 1.4$ & 16.5 & $1.45 \pm 0.26$ & $6.11 \pm 0.8$ & $2.90 \pm 0.23$ & $0.85 \pm 0.01$ & 0.042380 & 67.99980 & 1.076 \\
\hline Kepler-94c & $104.10^{\mathrm{h}}$ & $820.3 \pm 3$ & $\ldots$ & $3126 \pm 200$ & 4321 & $\ldots$ & $262.7 \pm 13.9$ & $\ldots$ & & $\ldots$ & $\ldots$ & $\ldots$ \\
\hline Kepler-103b & 108.01 & 15.9654 & $3.37 \pm 0.09$ & $9.7 \pm 8.6$ & 30.0 & $1.38 \pm 1.4$ & $2.32 \pm 2.1$ & $0.52 \pm 0.02$ & $0.72 \pm 0.01$ & 0.021500 & 75.17614 & 0.866 \\
\hline Kepler-103c & 108.02 & 179.612 & $5.14 \pm 0.14$ & $36.10 \pm 25.2$ & 95 & $1.47 \pm 1.2$ & $3.85 \pm 2.7$ & $0.52 \pm 0.02$ & $0.44 \pm 0.02$ & 0.032766 & 228.32581 & 0.824 \\
\hline Kepler-106c & 116.01 & 13.5708 & $2.50 \pm 0.32$ & $10.44 \pm 3.2$ & 18.8 & $3.28 \pm 1.56$ & $2.71 \pm 0.8$ & $1.38 \pm 0.15$ & $0.69 \pm 0.03$ & 0.022113 & 69.27837 & 1.000 \\
\hline Kepler-106e & 116.02 & 43.8445 & $2.56 \pm 0.33$ & $11.17 \pm 5.8$ & 24.8 & $3.10 \pm 2.07$ & $1.95 \pm 1.0$ & $1.38 \pm 0.15$ & $0.28 \pm 0.13$ & 0.022676 & 84.93360 & 0.933 \\
\hline Kepler-106b & 116.03 & 6.16486 & $0.82 \pm 0.11$ & $0.15 \pm 2.8$ & 5.3 & $1.26 \pm 26.26$ & $0.05 \pm 0.9$ & $1.38 \pm 0.15$ & $0.39 \pm 0.15$ & 0.007258 & 68.64035 & 0.992 \\
\hline Kepler-106d & 116.04 & 23.9802 & $0.95 \pm 0.13$ & $-6.39 \pm 7.0$ & 8.1 & $-41.00 \pm 39.75$ & $-2.84 \pm 0.9$ & $1.38 \pm 0.15$ & $0.45 \pm 0.11$ & 0.008362 & 80.53263 & 1.051 \\
\hline Kepler-95b & 122.01 & 11.5231 & $3.42 \pm 0.09$ & $13.0 \pm 2.9$ & 16.7 & $1.71 \pm 0.37$ & $3.36 \pm 0.6$ & $0.54 \pm 0.02$ & $0.74 \pm 0.01$ & 0.022166 & 64.96841 & 0.891 \\
\hline Kepler-109b & 123.01 & 6.48163 & $2.37 \pm 0.07$ & $1.3 \pm 5.4$ & 7.3 & $0.3 \pm 2.2$ & $0.29 \pm 1.8$ & $0.65 \pm 0.02$ & $0.58 \pm 0.02$ & 0.016434 & 55.97755 & 1.000 \\
\hline Kepler-109c & 123.02 & 21.2227 & $2.52 \pm 0.07$ & $2.22 \pm 7.8$ & 21.8 & $0.65 \pm 2.30$ & $0.43 \pm 1.5$ & $0.65 \pm 0.02$ & $0.16 \pm 0.08$ & 0.017432 & 70.57250 & 0.975 \\
\hline Kepler-48b & 148.01 & 4.77800 & $1.88 \pm 0.10$ & $3.94 \pm 2.1$ & 13.0 & $3.23 \pm 1.84$ & $1.62 \pm 0.9$ & $1.98 \pm 0.13$ & $0.17 \pm 0.11$ & 0.019318 & 57.06113 & 0.933 \\
\hline Kepler-48c & 148.02 & 9.67395 & $2.71 \pm 0.14$ & $14.61 \pm 2.3$ & 22.7 & $4.01 \pm 0.91$ & $4.74 \pm 0.7$ & $1.98 \pm 0.13$ & $0.38 \pm 0.05$ & 0.027892 & 58.33925 & 0.992 \\
\hline Kepler-48d & 148.03 & 42.8961 & $2.04 \pm 0.11$ & $7.93 \pm 4.6$ & 25.0 & $5.08 \pm 3.12$ & $1.57 \pm 0.9$ & $1.98 \pm 0.13$ & $0.20 \pm 0.11$ & 0.021021 & 79.06554 & 0.976 \\
\hline Kepler-48e & 148.10 & $982 \pm 8$ & $\ldots$ & $657 \pm 25$ & 727 & $\ldots$ & $45.83 \pm 0.8$ & $\ldots$ & $\ldots$ & $\ldots$ & $\ldots$ & $\ldots$ \\
\hline Kepler-113c & $153.01^{\mathrm{i}}$ & 8.92507 & $2.18 \pm 0.06$ & $-4.04 \pm 6.4$ & 8.7 & $-2.13 \pm 3.4$ & $-1.52 \pm 2.4$ & $2.71 \pm 0.08$ & $0.56 \pm 0.02$ & 0.029135 & 72.71374 & 0.950 \\
\hline Kepler-113b & $153.02^{\mathrm{i}}$ & 4.75400 & $1.82 \pm 0.05$ & $11.7 \pm 4.2$ & 20. & $10.73 \pm 3.9$ & $5.4 \pm 1.9$ & $2.71 \pm 0.08$ & $0.08 \pm 0.06$ & 0.024190 & 61.54630 & 0.950 \\
\hline Kepler-25c & 244.01 & 12.7204 & $5.20 \pm 0.09$ & $24.60 \pm 5.7$ & 32.4 & $0.90 \pm 0.21$ & $5.63 \pm 1.3$ & $0.75 \pm 0.01$ & $0.88 \pm 0.00$ & 0.036409 & 111.52730 & 1.127 \\
\hline Kepler-25b & 244.02 & 6.2385 & $2.71 \pm 0.05$ & $9.6 \pm 4.2$ & 16.0 & $2.50 \pm 1.10$ & $2.80 \pm 1.2$ & $0.75 \pm 0.01$ & $0.54 \pm 0.01$ & 0.018950 & 104.70541 & 1.051 \\
\hline Kepler-25d & $244.10^{\mathrm{h}}$ & $123 \pm 2$ & $\ldots$ & $89.90 \pm 13.7$ & 101 & $\ldots$ & $9.67 \pm 1.5$ & $\ldots$ & & $\ldots$ & $\ldots$ & $\ldots$ \\
\hline Kepler-37d & 245.01 & 39.7922 & $1.94 \pm 0.06$ & $1.87 \pm 9.08$ & 12.2 & $1.30 \pm 3.67$ & $0.40 \pm 1.2$ & $2.46 \pm 0.04$ & $0.53 \pm 0.01$ & 0.023068 & 108.24950 & 1.160 \\
\hline Kepler-37c & 245.02 & 21.3020 & $0.75 \pm 0.03$ & $3.35 \pm 4.0$ & 12.0 & $44.33 \pm 53.60$ & $0.92 \pm 1.1$ & $2.46 \pm 0.04$ & $0.43 \pm 0.02$ & 0.008909 & 124.83685 & 1.227 \\
\hline Kepler-37b & 245.03 & 13.3675 & $0.32 \pm 0.02$ & $2.78 \pm 3.7$ & 10.0 & $548.8 \pm 700.0$ & $0.95 \pm 1.1$ & $2.46 \pm 0.04$ & $0.48 \pm 0.13$ & 0.003828 & 117.04171 & 0.950 \\
\hline Kepler-68b & 246.01 & 5.39875 & $2.33 \pm 0.02$ & $5.97 \pm 1.7$ & 9.3 & $2.60 \pm 0.74$ & $2.07 \pm 0.59$ & $0.79 \pm 0.01$ & $0.48 \pm 0.01$ & 0.017383 & 106.85783 & 1.076 \\
\hline Kepler-68c & 246.02 & 9.60504 & $1.00 \pm 0.02$ & $2.18 \pm 3.5$ & 7.2 & $10.77 \pm 17.29$ & $0.57 \pm 0.9$ & $0.79 \pm 0.01$ & $0.77 \pm 0.01$ & 0.007455 & 69.38025 & 1.084 \\
\hline Kepler-68d & $246.10^{\mathrm{h}}$ & $625 \pm 16$ & $\ldots$ & $267 \pm 16$ & 283 & $\ldots$ & $19.06 \pm 0.58$ & $\ldots$ & $\ldots$ & $\ldots$ & $\ldots$ & $\ldots$ \\
\hline Kepler-96b & 261.01 & 16.2385 & $2.67 \pm 0.22$ & $8.46 \pm 3.4$ & 12.0 & $2.26 \pm 1.11$ & $2.10 \pm 0.8$ & $1.54 \pm 0.25$ & $0.54 \pm 0.07$ & 0.023967 & 104.01897 & 1.219 \\
\hline Kepler-131b & 283.01 & 16.0920 & $2.41 \pm 0.20$ & $16.13 \pm 3.5$ & 19.4 & $\mathbf{6 . 0 0} \pm \mathbf{1 . 9 8}$ & $3.95 \pm 0.8$ & $1.44 \pm 0.21$ & $0.79 \pm 0.02$ & 0.021263 & 103.59795 & 1.051 \\
\hline Kepler-131c & 283.02 & 25.5169 & $0.84 \pm 0.07$ & $8.25 \pm 5.9$ & 20.0 & $77.7 \pm 55$ & $3.58 \pm 0.9$ & $1.44 \pm 0.21$ & $0.27 \pm 0.16$ & 0.007394 & 87.42342 & 0.891 \\
\hline
\end{tabular}


Table 2

(Continued)

\begin{tabular}{|c|c|c|c|c|c|c|c|c|c|c|c|c|}
\hline Kepler No. & KOI & $\begin{array}{r}\text { Period }^{\mathrm{a}} \\
\text { (days) }^{2}\end{array}$ & $\begin{array}{l}\text { Radius }^{\mathrm{b}} \\
\left(R_{\oplus}\right)\end{array}$ & $\begin{array}{c}\operatorname{Mass}(\text { peak })^{\mathrm{c}} \\
\quad\left(M_{\oplus}\right)\end{array}$ & $\begin{array}{c}\text { Mass }^{\mathrm{d}} \\
95 \%\left(M_{\oplus}\right)\end{array}$ & $\begin{array}{l}\text { Planet Density } \\
\quad\left(\mathrm{g} \mathrm{cm}^{-3}\right)\end{array}$ & $\begin{array}{c}K^{\mathrm{f}} \\
\left(\mathrm{m} \mathrm{s}^{-1}\right)\end{array}$ & $\begin{array}{l}\text { Stellar Density } \\
\quad\left(\mathrm{g} \mathrm{cm}^{-3}\right)\end{array}$ & $\begin{array}{c}\text { Impact } \\
\text { Param. }(b)\end{array}$ & $R_{\mathrm{P}} / R_{*}$ & $\begin{array}{c}\text { Midtransit } E \\
\text { (BJD-2454900) }\end{array}$ & Reduced $\mathrm{Chi}^{2}$ \\
\hline Kepler-97b & 292.01 & 2.58664 & $1.48 \pm 0.13$ & $3.51 \pm 1.9$ & 9.1 & $5.44 \pm 3.48$ & $1.65 \pm 0.9$ & $1.53 \pm 0.31$ & $0.41 \pm 0.16$ & 0.013803 & 104.84121 & 0.950 \\
\hline Kepler-97c & $292.10^{\mathrm{g}}$ & $>789$ & & $>344$ & & & $>25$ & & & & & \\
\hline Kepler-98b & 299.01 & 1.54168 & $1.99 \pm 0.22$ & $3.55 \pm 1.6$ & 6.4 & $2.18 \pm 1.21$ & $1.82 \pm 0.8$ & $1.12 \pm 0.34$ & $0.56 \pm 0.14$ & 0.016377 & 103.54328 & 0.950 \\
\hline Kepler-99b & 305.01 & 4.60358 & $1.48 \pm 0.08$ & $6.15 \pm 1.3$ & 9.7 & $10.90 \pm 2.82$ & $2.91 \pm 0.6$ & $2.86 \pm 0.23$ & $0.21 \pm 0.13$ & 0.018475 & 104.83893 & 0.992 \\
\hline Kepler-406b & 321.01 & 2.42629 & $1.43 \pm 0.03$ & $6.35 \pm 1.4$ & 8.1 & $11.82 \pm 2.70$ & $2.89 \pm 0.6$ & $1.12 \pm 0.02$ & $0.10 \pm 0.06$ & 0.012285 & 103.45680 & 1.093 \\
\hline Kepler-406c & 321.02 & 4.62332 & $0.85 \pm 0.03$ & $2.71 \pm 1.8$ & 6.0 & $24.39 \pm 16.13$ & $1.00 \pm 0.6$ & $1.12 \pm 0.02$ & $0.36 \pm 0.06$ & 0.007261 & 65.37438 & 0.883 \\
\hline Kepler-407b & 1442.01 & 0.669310 & $1.07 \pm 0.02$ & $0.06 \pm 1.2$ & 3.2 & $0.29 \pm 5.70$ & $0.05 \pm 0.9$ & $1.38 \pm 0.16$ & $0.25 \pm 0.15$ & 0.010414 & 67.13162 & 0.958 \\
\hline Kepler-407c & $1442.10^{\mathrm{g}}$ & $3000 \pm 500$ & & $4000 \pm 2000$ & 8000 & & $164 \pm 20$ & $\ldots$ & & & $\ldots$ & $\ldots$ \\
\hline & 1612.01 & 2.46502 & $0.82 \pm 0.03$ & $0.48 \pm 3.2$ & 5.0 & $4.42 \pm 29.82$ & $0.20 \pm 1.3$ & $0.82 \pm 0.01$ & $0.92 \pm 0.01$ & 0.006136 & 65.67928 & 0.849 \\
\hline Kepler-409b & 1925.01 & 68.9584 & $1.19 \pm 0.03$ & $2.69 \pm 6.2$ & 22.0 & $8.88 \pm 20.60$ & $0.45 \pm 1.0$ & $1.81 \pm 0.00$ & $0.90 \pm 0.01$ & 0.012223 & 112.08151 & 1.059 \\
\hline
\end{tabular}

Notes.

a Each planet's orbital period is determined using only the Kepler photometry.

${ }^{\mathrm{b}}$ Each planet's radius is measured using the combined RV/photometry analysis, consistent with the best values found for the stellar mass and radius. Errors are dominated by uncertainties in the stellar radius.

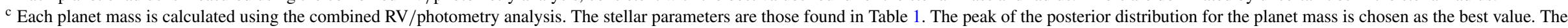
peak can have either a positive or negative value.

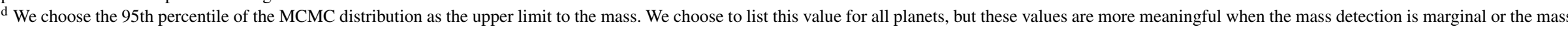
is poorly constrained.

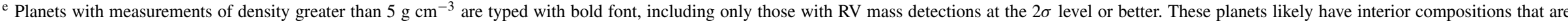

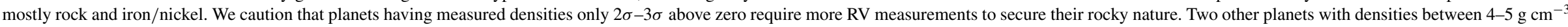
are also likely rocky, namely Kepler-102e and Kepler-48c.

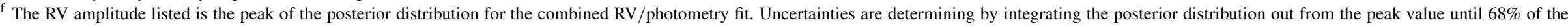
values in the MCMC chain are included.

$\mathrm{g}$ Long term variation in RVs indicates a long period orbiting object, which may not be a planet (Marcy et al. 2001).

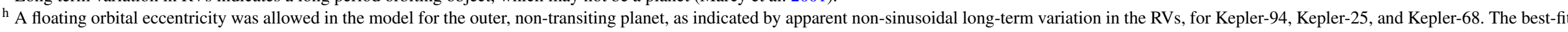
eccentricities are $0.38 \pm 0.05,0.18 \pm 0.10$, and $0.10 \pm 0.04$, respectively. See notes in Section 7 on the individual KOIs for more information.

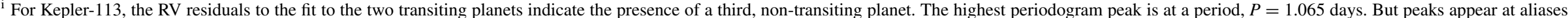

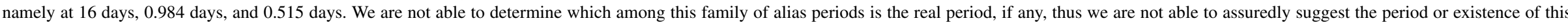
prospective non-transiting planet around Kepler-113. 
Table 3

False-positive Probabilities

\begin{tabular}{|c|c|c|c|c|c|c|c|c|c|}
\hline Kepler No. & KOI & $r_{\text {Excl. }}["]^{\mathrm{a}}$ & Comp. Sep.[“] b & $P_{\mathrm{EB}}$ & $P_{\mathrm{HEB}}$ & $P_{\mathrm{BGEB}}$ & $P_{\mathrm{BGPL}}$ & $\operatorname{Prior}_{\mathrm{PL}}{ }^{\mathrm{c}}$ & $\mathrm{FPP}^{\mathrm{d}}$ \\
\hline Kepler-100c & 41.01 & 4-SAT & Single & $<1 \mathrm{e}-4$ & $<1 \mathrm{e}-4$ & $<1 \mathrm{e}-4$ & $<1 \mathrm{e}-4$ & 0.56 & $<1 \mathrm{e}-4$ \\
\hline Kepler-100b & 41.02 & 4-SAT & Single & $<1 \mathrm{e}-4$ & $<1 \mathrm{e}-4$ & $<1 \mathrm{e}-4$ & $<1 \mathrm{e}-4$ & 0.25 & $<1 \mathrm{e}-4$ \\
\hline Kepler-100d & 41.03 & 4-SAT & Single & $<1 \mathrm{e}-4$ & $<1 \mathrm{e}-4$ & $<1 \mathrm{e}-4$ & $<1 \mathrm{e}-4$ & 0.37 & $<1 \mathrm{e}-4$ \\
\hline Kepler-93b & 69.01 & 4-SAT & Single & $<1 \mathrm{e}-4$ & $<1 \mathrm{e}-4$ & $<1 \mathrm{e}-4$ & $<1 \mathrm{e}-4$ & 0.33 & $<1 \mathrm{e}-4$ \\
\hline Kepler-102e & 82.01 & 4-SAT & Single & $<1 \mathrm{e}-4$ & $<1 \mathrm{e}-4$ & $<1 \mathrm{e}-4$ & $<1 \mathrm{e}-4$ & 0.56 & $<1 \mathrm{e}-4$ \\
\hline Kepler-102d & 82.02 & 4-SAT & Single & $<1 \mathrm{e}-4$ & $<1 \mathrm{e}-4$ & $<1 \mathrm{e}-4$ & $<1 \mathrm{e}-4$ & 0.18 & $<1 \mathrm{e}-4$ \\
\hline Kepler-102f & 82.03 & 4-SAT & Single & $<1 \mathrm{e}-4$ & $<1 \mathrm{e}-4$ & $<1 \mathrm{e}-4$ & $<1 \mathrm{e}-4$ & 0.07 & $<1 \mathrm{e}-4$ \\
\hline Kepler-102c & 82.04 & 4-SAT & Single & $<1 \mathrm{e}-4$ & $<1 \mathrm{e}-4$ & $2.5 \mathrm{e}-4$ & $0.89 \mathrm{e}-4$ & 0.01 & $3.4 \mathrm{e}-4$ \\
\hline Kepler-102b & 82.05 & 4-SAT & Single & $<1 \mathrm{e}-4$ & $<1 \mathrm{e}-4$ & $7.6 e-4$ & $0.2 \mathrm{e}-4$ & 0.00 & $7.8 \mathrm{e}-4$ \\
\hline Kepler-94b & 104.01 & 0.035 & Single & $<1 \mathrm{e}-4$ & $<1 \mathrm{e}-4$ & $<1 \mathrm{e}-4$ & $<1 \mathrm{e}-4$ & 0.34 & $<1 \mathrm{e}-4$ \\
\hline Kepler-103b & 108.01 & 0.009 & $2.44,4.87$ & $<1 \mathrm{e}-4$ & $<1 \mathrm{e}-4$ & $<1 \mathrm{e}-4$ & $<1 \mathrm{e}-4$ & 0.37 & $<1 \mathrm{e}-4$ \\
\hline Kepler-103c & 108.02 & 0.04 & $2.44,4.87$ & $<1 \mathrm{e}-4$ & $1 \mathrm{e}-4$ & $<1 \mathrm{e}-4$ & $<1 \mathrm{e}-4$ & 0.13 & $1 e-4$ \\
\hline Kepler-106c & 116.01 & 0.23 & Single & $<1 \mathrm{e}-4$ & $<1 \mathrm{e}-4$ & $<1 \mathrm{e}-4$ & $<1 \mathrm{e}-4$ & 0.55 & $<1 \mathrm{e}-4$ \\
\hline Kepler-106e & 116.02 & 0.49 & Single & $<1 \mathrm{e}-4$ & $<1 \mathrm{e}-4$ & $<1 \mathrm{e}-4$ & $<1 \mathrm{e}-4$ & 0.53 & $<1 \mathrm{e}-4$ \\
\hline Kepler-106b & 116.03 & 0.83 & Single & $<1 \mathrm{e}-4$ & $<1 \mathrm{e}-4$ & $<1 \mathrm{e}-4$ & $<1 \mathrm{e}-4$ & 0.05 & $<1 \mathrm{e}-4$ \\
\hline Kepler-106d & 116.04 & 2.1 & Single & $<1 \mathrm{e}-4$ & $<1 \mathrm{e}-4$ & $0.2 \mathrm{e}-4$ & $1.4 \mathrm{e}-4$ & 0.09 & $1.7 e-4$ \\
\hline Kepler-95b & 122.01 & 0.12 & 4.1 & $<1 \mathrm{e}-4$ & $<1 \mathrm{e}-4$ & $<1 \mathrm{e}-4$ & $<1 \mathrm{e}-4$ & 0.37 & $<1 \mathrm{e}-4$ \\
\hline Kepler-109b & 123.01 & 0.11 & $2.03,5.3$ & $<1 \mathrm{e}-4$ & $<1 \mathrm{e}-4$ & $<1 \mathrm{e}-4$ & $<1 \mathrm{e}-4$ & 0.56 & $<1 \mathrm{e}-4$ \\
\hline Kepler-109c & 123.02 & 0.09 & $2.03,5.3$ & $<1 \mathrm{e}-4$ & $<1 \mathrm{e}-4$ & $<1 \mathrm{e}-4$ & $<1 \mathrm{e}-4$ & 0.55 & $<1 \mathrm{e}-4$ \\
\hline Kepler-48b & 148.01 & 0.16 & $2.44^{\mathrm{e}}$ & $<1 \mathrm{e}-4$ & $<1 \mathrm{e}-4$ & $<1 \mathrm{e}-4$ & $<1 \mathrm{e}-4$ & 0.54 & $<1 \mathrm{e}-4$ \\
\hline Kepler-48c & 148.02 & 0.26 & $2.44^{\mathrm{e}}$ & $<1 \mathrm{e}-4$ & $<1 \mathrm{e}-4$ & $<1 \mathrm{e}-4$ & $<1 \mathrm{e}-4$ & 0.45 & $<1 \mathrm{e}-4$ \\
\hline Kepler-48d & 148.03 & 0.28 & $2.44^{\mathrm{e}}$ & $<1 \mathrm{e}-4$ & $<1 \mathrm{e}-4$ & $<1 \mathrm{e}-4$ & $<1 \mathrm{e}-4$ & 0.56 & $<1 \mathrm{e}-4$ \\
\hline Kepler-113c & 153.01 & 0.14 & 5.14 & $<1 \mathrm{e}-4$ & $<1 \mathrm{e}-4$ & $<1 \mathrm{e}-4$ & $<1 \mathrm{e}-4$ & 0.56 & $<1 \mathrm{e}-4$ \\
\hline Kepler-113d & 153.02 & 0.09 & 5.14 & $<1 \mathrm{e}-4$ & $<1 \mathrm{e}-4$ & $<1 \mathrm{e}-4$ & $<1 \mathrm{e}-4$ & 0.47 & $<1 \mathrm{e}-4$ \\
\hline Kepler-25c & 244.01 & 4-SAT & Single & $<1 \mathrm{e}-4$ & $<1 \mathrm{e}-4$ & $<1 \mathrm{e}-4$ & $<1 \mathrm{e}-4$ & 0.12 & $<1 \mathrm{e}-4$ \\
\hline Kepler-25b & 244.02 & 4-SAT & Single & $<1 \mathrm{e}-4$ & $<1 \mathrm{e}-4$ & $<1 \mathrm{e}-4$ & $<1 \mathrm{e}-4$ & 0.51 & $<1 \mathrm{e}-4$ \\
\hline Kepler-37d & 245.01 & 4-SAT & Single & $<1 \mathrm{e}-4$ & $<1 \mathrm{e}-4$ & $<1 \mathrm{e}-4$ & $<1 \mathrm{e}-4$ & 0.50 & $<1 \mathrm{e}-4$ \\
\hline Kepler-37c & 245.02 & 4-SAT & Single & $<1 \mathrm{e}-4$ & $<1 \mathrm{e}-4$ & $<1 \mathrm{e}-4$ & $<1 \mathrm{e}-4$ & 0.04 & $<1 \mathrm{e}-4$ \\
\hline Kepler-37b & 245.03 & 4-SAT & Single & $<1 \mathrm{e}-4$ & $<1 \mathrm{e}-4$ & 0.001 & $<1 \mathrm{e}-4$ & 0.01 & $4 e-4^{g}$ \\
\hline Kepler-68b & 246.01 & 4-SAT & Single & $<1 \mathrm{e}-4$ & $<1 \mathrm{e}-4$ & $<1 \mathrm{e}-4$ & $<1 \mathrm{e}-4$ & 0.55 & $<1 \mathrm{e}-4$ \\
\hline Kepler-68c & 246.02 & 4-SAT & Single & $<1 \mathrm{e}-4$ & $<1 \mathrm{e}-4$ & $6.2 \mathrm{e}-4$ & $<1 \mathrm{e}-4$ & 0.15 & $6.3 e-4$ \\
\hline Kepler-96b & 261.01 & 4-SAT & 5.4 & $<1 \mathrm{e}-4$ & $<1 \mathrm{e}-4$ & $<1 \mathrm{e}-4$ & $<1 \mathrm{e}-4$ & 0.53 & $<1 \mathrm{e}-4$ \\
\hline Kepler-131b & 283.01 & 4-SAT & 6 & $<1 \mathrm{e}-4$ & $<1 \mathrm{e}-4$ & $<1 \mathrm{e}-4$ & $<1 \mathrm{e}-4$ & 0.55 & $<1 \mathrm{e}-4$ \\
\hline Kepler-131c & 283.02 & 4-SAT & 6 & $<1 \mathrm{e}-4$ & $<1 \mathrm{e}-4$ & $9.8 \mathrm{e}-04$ & $<1 \mathrm{e}-4$ & 0.06 & $9.8 \mathrm{e}-4$ \\
\hline Kepler-97b & 292.01 & 0.20 & $0.38^{f}$ & $<1 \mathrm{e}-4$ & $<1 \mathrm{e}-4$ & $<1 \mathrm{e}-4$ & $<1 \mathrm{e}-4$ & 0.31 & $<1 \mathrm{e}-4$ \\
\hline Kepler-98b & 299.01 & 0.15 & Single & $<1 \mathrm{e}-4$ & $<1 \mathrm{e}-4$ & $<1 \mathrm{e}-4$ & $<1 \mathrm{e}-4$ & 0.52 & $<1 \mathrm{e}-4$ \\
\hline Kepler-99b & 305.01 & 0.18 & Single & $<1 \mathrm{e}-4$ & $<1 \mathrm{e}-4$ & $<1 \mathrm{e}-4$ & $<1 \mathrm{e}-4$ & 0.31 & $<1 \mathrm{e}-4$ \\
\hline Kepler-406b & 321.01 & 0.24 & Single & $<1 \mathrm{e}-4$ & $<1 \mathrm{e}-4$ & $<1 \mathrm{e}-4$ & $<1 \mathrm{e}-4$ & 0.30 & $<1 \mathrm{e}-4$ \\
\hline Kepler-406c & 321.02 & 0.20 & Single & $<1 \mathrm{e}-4$ & $<1 \mathrm{e}-4$ & $<1 \mathrm{e}-4$ & $<1 \mathrm{e}-4$ & 0.06 & $<1 \mathrm{e}-4$ \\
\hline Kepler-407b & 1442.01 & 0.33 & 2.1 & $<1 \mathrm{e}-4$ & $<1 \mathrm{e}-4$ & $<1 \mathrm{e}-4$ & $<1 \mathrm{e}-4$ & 0.14 & $<1 \mathrm{e}-4$ \\
\hline$\ldots$ & 1612.01 & 4-SAT & Single & $<1 \mathrm{e}-4$ & $<1 \mathrm{e}-4$ & $2.1 \mathrm{e}-2$ & $1.9 \mathrm{e}-4$ & 0.05 & 0.021 \\
\hline Kepler-409b & 1925.01 & 4-SAT & Single & $<1 \mathrm{e}-4$ & $<1 \mathrm{e}-4$ & $3.2 \mathrm{e}-4$ & $<1 \mathrm{e}-4$ & 0.18 & $3.2 \mathrm{e}-4$ \\
\hline
\end{tabular}

Notes.

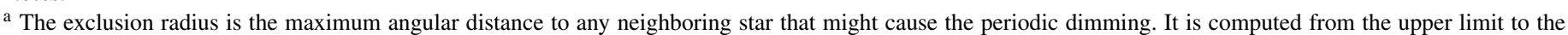

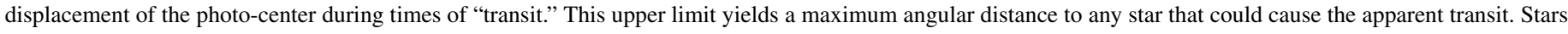
farther away cannot be responsible. The note 4-SAT denotes saturated images from Kepler, limiting astrometric accuracy to 4 " (one pixel).

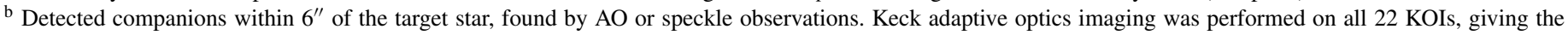

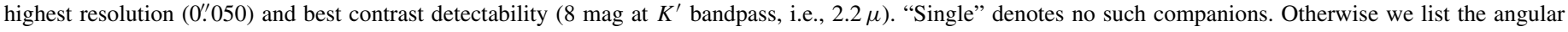

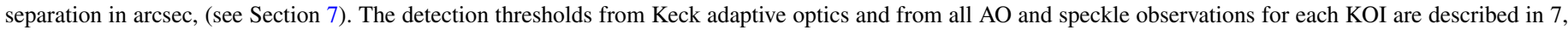
and plotted in Figures 1-44.

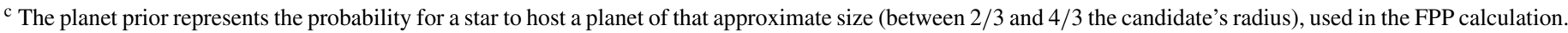

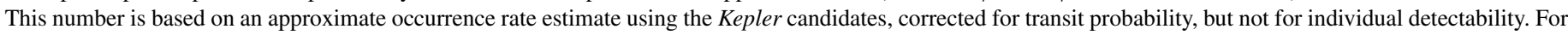

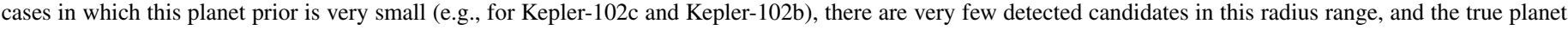
prior should be higher.

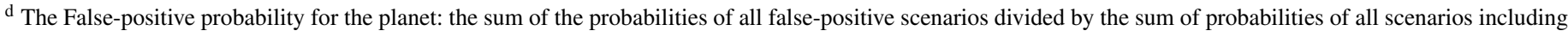
that the planet is real.

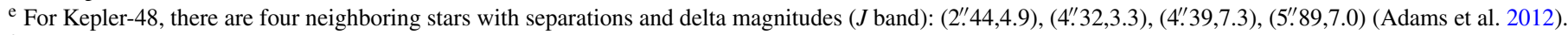

f Kepler-97: The neighbor $\left(0^{\prime \prime} 36\right)$ is farther than the astrometric maximum radius $\left(0{ }^{\prime \prime} 2\right)$. Thus, the planet probably does not orbit neighbor.

$\mathrm{g}$ Careful false-positive analysis by Barclay et al. (2013) shows FPP $=0.0004$. 
images with resolution of 0 .'05. This non-transiting companion has a designation of Kepler-93c and these values are listed in Table 2. It remains possible that the transiting planet orbits the unresolved massive companion rather than the primary star. If so, it would have to be Jupiter-size to yield the observed transit depth, with a period of 4.7 days, which would be a rarity. A more detailed analysis of Kepler-93 is carried out by S. Ballard et al. (2013, submitted).

We place upper limits on the mass of the transiting planet at 4.4 $M_{\oplus}$, which corresponds to a bulk density of $7.2 \mathrm{~g} \mathrm{~cm}^{-3}$. This is only an upper limit because the median value of the posterior distribution of the MCMC analysis of the planet mass is only slightly above zero, and the value is consistent with zero at the $2 \sigma$ level. The phase-folded RV curve shows the $K$ amplitude of $1.05 \pm 0.8 \mathrm{~m} \mathrm{~s}^{-1}$ (Figure 4 , bottom right), not significant at better than $1 \sigma$. The full set of RVs is plotted versus time in Figure 4 (top) and listed along with $\log R_{\mathrm{HK}}^{\prime}$ activity values in Table 4.

\subsection{KOI-82, Kepler-102}

The five planets in this system have periods of 5.28, 7.1, 10.3, 16.1, and 27.5 days and have corresponding radii of $0.5,0.6$, 1.0, 2.2 and $0.9 R_{\oplus}$. Discovered according to Kepler photometric detectability, their KOI numbers are not in order of increasing orbital period. Due to their very small transit depths of only 41 and 65 ppm, 82.04 and 82.05 were detected last, but have the shortest periods. The Kepler-102 letters are assigned to each planet by increasing orbital period.

Recon spectroscopy was first acquired with the Lick $3 \mathrm{~m}$ telescope in 2009 August. Two spectra taken four days apart gave stellar values in agreement with the KIC value for $T_{\text {eff }}$, but the value of $\log g=5.0$ conflicted with the KIC $\log g$ value of 4.0. The final stellar parameters determined with SME analysis combined with stellar models and light curve fits are $T_{\text {eff }}=$ $4903 \pm 74 \mathrm{~K}, \log g=4.61 \pm 0.03$ and $[\mathrm{Fe} / \mathrm{H}]=0.08 \pm 0.07$. The final $\log g$ value and stellar mass changed the KIC value of stellar radius from $1.8 R_{\odot}$ to $0.74 R_{\odot}$. The initial values of planet radii were likewise reduced by a factor of $\sim 2.5$.

Due to the intense interest in the system of five small planets, and the recon-derived stellar parameters that differed greatly from the KIC values, high-resolution spectra were taken at Keck/HIRES and FIES to confirm the stellar properties. Several trials of SME analysis and SPC analysis were made to robustly confirm the $T_{\text {eff }}, \log g$, and $[\mathrm{Fe} / \mathrm{H}]$. In the end all analyses agreed within $1 \sigma$ errors, given above.

Standard follow-up with speckle imaging in 2010 June and ARIES AO observations in 2010 September found no stellar companions within their detection domains, supporting the notion that the transits occur on the primary star or an unresolved bound companion star. Figure 5, top left, shows a seeing-limited image of the field of view from the Keck-HIRES guide camera. Figure 5 (middle panel) shows the limiting magnitudes achieved with each imaging method. Keck AO imaging on 2013 June $13 / 14$ and 14/15 yielded a PSF with FWHM of $0.0^{\prime} 05$ ', and detectability $7 \mathrm{mag}$ fainter than the primary star at $K^{\prime}$, for all separations more than 0.2 . The full detectability curve is given in Figure 5. The absence of any stellar companion in the Keck AO images greatly reduces the probability of any stellar neighbor that might pose an alternative to the transiting planet interpretation. This sense is confirmed with the detailed false-positive analysis (See Section 6). Confirmation of Kepler102e was performed independently by Wang et al. (2013) using
Table 4

Radial Velocities for Kepler-100

\begin{tabular}{lccc}
\hline \hline BJD - 2450000 & $\begin{array}{c}\text { Radial Velocity } \\
\left(\mathrm{m} \mathrm{s}^{-1}\right)\end{array}$ & $\begin{array}{c}\text { Uncertainty } \\
\left(\mathrm{m} \mathrm{s}^{-1}\right)\end{array}$ & $\log R_{\mathrm{HK}}^{\prime}$ \\
\hline 4989.010954 & -4.46 & 3.73 & -5.028 \\
5042.015300 & -11.82 & 3.81 & -5.034 \\
5042.022823 & -9.46 & 3.86 & -5.028 \\
5042.030068 & -6.02 & 3.77 & -5.041 \\
5042.842452 & -3.45 & 3.63 & -5.041 \\
\hline
\end{tabular}

(This table is available in its entirety in a machine-readable form in the online journal. A portion is shown here for guidance regarding its form and content.)

pixel centroid offsets, transit depth measurements and a UKIRT constrast curve.

With five transiting planets having orbital periods less than 25 days and planet radii less than $2.5 R_{\oplus}$, Kepler-102 appears to be a densely packed, rich system of small planets. The RV time baseline of nearly 900 days shows only a $4.3 \mathrm{~m} \mathrm{~s}^{-1}$ scatter. We fit the RVs to the orbits specified by the transit ephemerides, using circular models (as with all transiting planets in this paper), see (Figure 6, top). We fit all five planets simultaneously with circular orbits. The 16 day planet (Kepler-102e) shows clear coherence with the RVs. We measure the planet mass at $8.9 \pm$ $2.0 M_{\oplus}$ (radius $2.22 R_{\oplus}$ ), and a density of $4.68 \pm 1.1 \mathrm{~g} \mathrm{~cm}^{-3}$. The RV semi-amplitude, $K$, is $2.77 \pm 0.6 \mathrm{~m} \mathrm{~s}^{-1}$, the largest for any planet in the system. The four remaining planets in the system do not appear in the RVs convincingly. Their induced $\mathrm{RV}$ variations are apparently at or below their respective RV detection thresholds, and we report $2 \sigma$ upper limits to their masses in Table 2. With the 3 yr time baseline, we constrain the presence of non-transiting planets out to 5 au with masses down to $\sim 1 M_{\text {JUP }}$. No detectable trends or periodic signals are seen in the RV residuals to the five planet fit. The full set of RVs are listed, along with their $\log R_{\mathrm{HK}}^{\prime}$ activity values, in Table 4.

\subsection{KOI-104, Kepler-94}

The Kepler photometry revealed a single transiting planet candidate orbiting Kepler-94 with a period of 2.50 days and radius (later found to be) $3.51 R_{\oplus}$. It resides in a multiple system, as precise RVs reveal the presence of a non-transiting orbiting companion with a period of $820 \pm 5$ days and $M \sin i$ of $9.8 \pm 0.6 M_{\mathrm{JUP}}$. Before the non-transiting planet was detected, we acquired the usual suite of follow-up observations. Recon spectroscopy was first done with the $2.6 \mathrm{~m}$ Nordic Optical Telescope in 2009 August. The three spectra that were acquired showed no RV variation above the errors of $200 \mathrm{~m} \mathrm{~s}^{-1}$, indicating the host star to be a slowly rotating cool dwarf.

Initial photometric analysis based on KIC stellar parameters yielded a star and planet radius smaller by $20 \%$ than the final planet radius. This slowly rotating K-type host star was recognized as ideal for precise RV spectroscopy. SME analysis of the HIRES template spectrum and comparison to Yonsei-Yale stellar models refined the stellar properties to be $T_{\text {eff }}=$ $4781 \pm 98 \mathrm{~K}, \log g=4.59 \pm 0.04$ and $[\mathrm{Fe} / \mathrm{H}]=+0.34 \pm$ 0.07 , in agreement with the stellar parameters determined from the recon analysis. The final planet radius is $3.51 \pm 0.15 R_{\oplus}$, stemming from our standard MCMC modeling of light curve and RVs and after adopting the final stellar radius. Kepler-94 was analyzed by Muirhead et al. (2012), but the resulting parameters were found to be uncertain with their method, which 
is best suited for mid- to late $\mathrm{M}$ dwarfs having $T_{\text {eff }}<4000 \mathrm{~K}$. The final stellar parameters can be found in Table 1 .

The " $V$-shaped light curve" led the KFOP to adopt caution regarding the planet interpretation. The Kepler photometric diagnostics suggested a chance that this was a blend of two or more stars. Speckle imaging revealed no companions within its limits. Subsequent AO observations taken at Palomar further limited the presence of stellar companions to within $1^{\prime \prime} .0$ away from the primary. The concerns regarding the $V$-shaped transit and possible blend of other stars initially yielded an FPP of $10 \%$, prior to the acquisition of the Keck AO images. The Keck AO images of Kepler-94 greatly reduced the probability of background and bound stellar companions, as described in detail in Section 6.1, yielding a final FPP of $<0.0001$, validating the planet.

Moreover, the RV periodicity is in phase with the Kepler ephemeris of this 2.51 days planet, adding further support to the reality of the transiting planet, and limiting the plausible falsepositive scenarios that would insidiously mimic both the light curve and RVs of a transiting planet. For a detailed description of the FPP calculation, see Section 6.2 and 6.3.

A seeing-limited image of the field of view from the KeckHIRES guider is found in Figure 7 (top left) while Figure 7 (middle) shows the limiting magnitudes achieved with each high-resolution imaging method. No stellar companions were found. Keck AO imaging on 2013 June 13/14 and 14/15 yielded a FWHM of 0 .'05. No companion was seen.

The first Keck-HIRES RV was acquired in 2010 June. The long baseline of the RVs, spanning 800 days (Figure 8, top), was vital for mapping out the orbit of the non-transiting planet. The precise RVs are in phase with the transiting planet in a circular orbit, and we measure the minimum planet mass $(M \sin i)$ to be $10.8 \pm 1.4 M_{\oplus}$. The transiting planet mass, combined with the planet radius measurement from Kepler yields a bulk density of $1.45 \pm 0.26 \mathrm{~g} \mathrm{~cm}^{-3}$, which is consistent with theoretical expectations by Lopez et al. (2012). This is a low-density planet composed of a large fraction of non-rocky material, perhaps $\mathrm{H}$ and He. While the planet radius and mass quoted above stems from a circular orbit model, the transiting planet appears to be in a non-circular orbit, despite its short period. It is one of the few cases in which a non-zero eccentricity is called for, allowing a better fit to the RVs than the circular orbit. The higher RV variation is due to the non-transiting planet with its period of 820 days, and we measure $M \sin i$ of that planet to be $9.8 \pm$ $0.6 M_{\mathrm{JUP}}$. This non-transiting planet has a non-circular orbit, and we measure the eccentricity to be $e=0.38 \pm 0.05$. The phase-folded RV curves are shown in Figure 8 (bottom right).

The eccentricity of the short period planet is unusual. Perhaps the non-transiting planet is pumping the eccentricity of the transiting planet. It is worth noting that there is only one transiting planet, perhaps because the non-transiting planet has scattered other planets out of the original protoplanetary plane. The most likely true mass of the non-transiting planet 10-20 $M_{\mathrm{JUP}}$, accounting for likely orbital inclinations, and the actual planet mass could be higher. The full set of RVs are listed, along with $\log R_{\mathrm{HK}}^{\prime}$ values, in Table 4 . It could be fruitful to search carefully for transit timing variations (TTVs) in the transit times of the inner planet.

\subsection{KOI-108, Kepler-103}

The two planets identified by Kepler photometry around Kepler-103 have periods of 15.97 and 179.6 d, with planet radii of 3.37 and $5.14 R_{\oplus}$, respectively. Follow-up spectroscopy at the McDonald $2.7 \mathrm{~m}$ in 2009 August were found to be in marginal agreement with the KIC estimates of $T_{\text {eff }}$ and $\log g$, and rotational $v \sin i<4 \mathrm{~km} \mathrm{~s}^{-1}$. These stellar properties, and its brightness at $K p=12.3$, warranted high-resolution spectroscopy at the Keck Observatory. SME analysis was conducted on a Keck-HIRES template spectrum. The results were used as initial guesses in the asteroseismology analysis, which determined the final stellar parameters, notably increasing the stellar radius by $25 \%$ from the KIC radius to $1.43 R_{\odot}$. The derived stellar parameters are $T_{\text {eff }}=5845 \pm 88 \mathrm{~K}, \log g=$ $4.16 \pm 0.04$, and $[\mathrm{Fe} / \mathrm{H}]=0.07 \pm 0.1$. The lack of $\mathrm{RV}$ measurements in 2012 is due to the refined stellar parameters, which increased the planet radii to $>3.0 R_{\oplus}$, diminishing its priority.

Keck AO imaging on 2013 June 13/14 and 14/15 yielded images with PSFs having a FWHM of 0 '.05. The detectability curve for neighboring stars is given in Figure 9. No companion was seen. AO imaging was also acquired in the $J$ band at Palomar Observatory, and two nearby stars were found separated by 2 ."44 and 4 ". 87 from the primary, both being 7.2 mag fainter than the primary. No companion stars were found within the limits of speckle imaging, taken in 2010 June, which probes from 0'.05 to 1 .". from the primary. During Keck-HIRES observations, the 4."87 stellar companion was purposely kept out of the HIRES slit, however the 2 .' 76 companion was not visible on the HIRES guider and some of its light may have inadvertently gone into the slit of the spectrometer. The HIRES slit is only $0^{\prime \prime} 87$ wide, so for most orientations of the rotating HIRES field of view (due to use of the image rotator) that nearby star would not fall in the slit. If it did, then the flux would be $<1 \%$ the brightness of the primary at optical wavelengths. For all of these reasons, we suspect any contamination from the 2 . 76 companion is negligible in the spectroscopy of the primary star, given the $\mathrm{S} / \mathrm{N}$ of $\sim 100$. Figure 9 (top left) shows a seeing-limited image of the field of view of the HIRES guider camera. Figure 9 (middle) shows the limiting magnitudes achieved with each high-resolution imaging method.

The second transit of the 180 days planet was found only after a full year of Kepler data was analyzed. Upon discovery of the first transit event of the 180 days planet, centroid analysis was conducted. Initially centroid motion seemed apparent, indicating a source location for the transit located 0.7 from the primary star, albeit at the $2 \sigma$ level. After more Kepler photometry was processed, the astrometric displacement was not confirmed, and the centroid analysis was found to be consistent with a transit on the target star.

The time baseline for the RVs spans 735 days (Figure 10, top). The RV signal does not strongly correlate with the orbital period of 16 days for the inner planet. The nominal mass of the 15 day planet is measured to be $9.7 \pm 8.6 M_{\oplus}$, corresponding to a bulk density of $1.38 \pm 1.4 \mathrm{~g} \mathrm{~cm}^{-3}$ when combined with the planet radius of $3.37 R_{\oplus}$. The RV signal has a semi-amplitude of $2.32 \pm 2.1 \mathrm{~m} \mathrm{~s}^{-1}$, providing only an upper limit to the mass. The 95th percentile of the posterior mass distribution gives the upper limit to the planet mass at $30 M_{\oplus}$. Since RV measurements are planned to occur at the predicted times of quadrature, the phase coverage of the RVs is poor and the eccentricity is not well constrained, leading us to conduct these fits using circular orbits.

For the 180 day planet, orbital analysis of the RVs yields a peak of the posterior distribution of masses to be at $3.85 \mathrm{~m} \mathrm{~s}^{-1}$, but with a large uncertainty of $2.7 \mathrm{~m} \mathrm{~s}^{-1}$. Poor phase coverage of the RVs for this long period planet make upper mass limit only 
marginally useful. When the RV is measured at both quadratures, both high and low, the mass limits will be more robust. The RVs are listed, along with their $\log R_{\mathrm{HK}}^{\prime}$ activity values, in Table 4. This system of two planets should be examined carefully for TTV signals.

\subsection{KOI-116, Kepler-106}

Kepler identified a quartet of candidate transiting planets around Kepler-106, with orbital periods of 6.2, 13.6, 24.0, and 43.8 days and planet radii of $0.8,2.5,0.95$ and $2.6 R_{\oplus}$, respectively. The two largest planets were identified within the first three months of Kepler data. Kepler-106b required quarters 1-6 of Kepler data, and the Kepler-106d required quarters $1-8$. The longer time baselines of the Kepler photometry were required to find such small signals.

Ground based follow-up observing started with acquisition of recon spectra using the $2.6 \mathrm{~m}$ Nordic Optical Telescope in 2009 August and the McDonald $2.7 \mathrm{~m}$ in 2009 September. Analysis of these spectra gave $T_{\text {eff }}$ in agreement with the KIC, but $\log g$ was found to be nearer 4.5 (than 4.0 from the KIC), placing this star on the main sequence, not slightly evolved. The main effect of this change in gravity is the decrease of the stellar radius by $50 \%$ to $1.04 R_{\odot}$. The planet radii were likewise decreased from $5 R_{\oplus}$ to their final values stated above and in Table 2. SME analysis of a HIRES template spectrum was used in combination with Yonsei-Yale stellar isochrones to determine the final stellar parameters of $T_{\text {eff }}=5858 \pm 114 \mathrm{~K}, \log g=4.407 \pm 0.14$, and $[\mathrm{Fe} / \mathrm{H}]=-0.12 \pm 0.1$. (Table 1$)$.

Keck AO imaging on 2013 June 13/14 and 14/15 yielded a FWHM of 0. '05, with a full detectability curve given in Figure 11. No companion was seen, ruling out companions 8 mag fainter in $K^{\prime}$ band (or brighter) located beyond $0^{\prime \prime} .4$ from the primary star. Speckle imaging at WIYN taken in 2010 June and AO observation taken at ARIES in $K s$ band in late 2010, also showed no companion stars within their detection limits. Figure 11 (top left) shows a seeing-limited image of the field of view of the HIRES guide camera, and Figure 11 (top right) shows the Keck AO image. Figure 11 (middle) shows the limiting magnitudes achieved with each high-resolution imaging method.

While precise RV monitoring started in 2010, the discovery of the fourth planet, and the adjustment of all four planet radii to values below $2.5 R_{\oplus}$, motivated an increased RV cadence in the 2012 observing season. The RVs obtained during $1073 \mathrm{~d}$ (Figure 12, top) exhibit no evidence of non-transiting planets nor monotonic trends. We computed a self-consistent fourplanet model fit to the RV, assuming circular orbits (as with all transiting planets in this paper) and the Kepler orbital ephemeris. Those fits show no evidence of the 6 days and 24 days planets in phased plots of the RVs (each plot having the remaining three planets removed).

The phased RVs for the planet (Kepler-106c) with a period of 13.57 days and radius $2.5 R_{\oplus}$ do correlate with the RVs predicted from the ephemeris, as shown in Figure 12, lower right. That RV signal yields a planet mass of $10.4 \pm 3 M_{\oplus}$, a $3 \sigma$ detection of the planet. This planet mass and radius corresponds to a planet density of $3.3 \pm 1.6 \mathrm{~g} \mathrm{~cm}^{-3}$.

Finally, Kepler-106e with $P=43 \mathrm{~d}$ and $R_{p}=2.6 R_{\oplus}$, shows only a weak correlation with the RVs, enabling a constraint on the planet mass of $11.2 \pm 6 M_{\oplus}$, below a $2 \sigma$ detection, and density $=3.1 \pm 2.1 \mathrm{~g} \mathrm{~cm}^{-3}$. While we cannot place useful upper limits on the masses or densities of the two sub-Earth radii planets, the RVs are consistent with the four planet system. The phase-folded RV curves for each planet are shown in Figure 12 (bottom right). The full set of RVs are listed, along with their $\log R_{\mathrm{HK}}^{\prime}$ activity values in Table 4 .

\subsection{KOI-122, Kepler-95}

Kepler-95 was identified by Kepler to have a single transiting planet with orbital period 11.5 days and radius $3.4 R_{\oplus}$. The first follow-up observations were two recon spectra taken in 2009 August at the McDonald $2.7 \mathrm{~m}$. The resulting measurements of $T_{\text {eff }}$ agree with the KIC value, but the $\log g$ value differs significantly, yielding an implied stellar radius of $1.41 R_{\odot}, 75 \%$ larger than the KIC value. We obtained a Keck-HIRES spectrum, and the SME analysis combined with asteroseismology (Huber et al. 2013) provides $\log g=4.17 \pm 0.04, T_{\text {eff }}=5699 \pm 74 \mathrm{~K}$ and $[\mathrm{Fe} / \mathrm{H}]=+0.30 \pm 0.1$ (see Table 1$)$. The corresponding adjustment to the planet radius (originally $1.9 R_{\oplus}$ ), moved this KOI out of the Kepler TCERT prioritized range of planet radii, i.e., above $3 R_{\oplus}$. This planet probably would not have been included in this survey, had we known the radii precisely to begin with.

Follow-up speckle imaging with the WIYN telescope in 2009 August found no stellar companions, but AO imaging at Palomar in 2010 June revealed a single companion star 4". 1 from the primary that is $6.5 \mathrm{mag}$ fainter in the $J$ band. Centroid analysis of this target rules out the possibility of the transit occurring on that neighboring star. Figure 13 (top left) shows a seeinglimited image of the field of view of the HIRES guide camera, in which the 4". 1 neighboring stars does not appear, presumably because it is so faint in the optical. Figure 13 (middle) shows the limiting magnitudes achieved with each high-resolution imaging method. Keck AO imaging on 2013 June 13/14 and 14/15 yielded a FWHM of 0 '.05, with a full detectability curve given in Figure 13. No additional companions were found beside that 4". 1 neighbor.

The precise RV time baseline from Keck-HIRES spans 1078 days and exhibits an rms of $5.1 \mathrm{~m} \mathrm{~s}^{-1}$ (see Figure 14, top). After fitting the ephemeris of the single planet, of radius $=3.4 R_{\oplus}$, to the RVs we detect the planet mass to be $13.0 \pm 2.9 M_{\oplus}$ with a corresponding density of $1.7 \pm 0.4 \mathrm{~g} \mathrm{~cm}^{-3}$. The RV detection is statistically significant, and the low density of $1.7 \mathrm{~g} \mathrm{~cm}^{-3}$ requires the planet to consist of a large fraction of volatiles by volume. This planet density is consistent with theoretical expectations for a planet of its size, $3-4 R_{\oplus}$, as noted in Table 4 of Lopez et al. (2012). The full set of RVs are listed, along with their $\log R_{\mathrm{HK}}^{\prime}$ activity values in Table 4.

\subsection{KOI-123, Kepler-109}

The Kepler-109 planetary system consists of two transiting planets with orbital periods of 6.5 days (Kepler-109b) and 21 days (Kepler-109c), and radii of 2.4 and $2.5 R_{\oplus}$, respectively. Two recon-level spectra were acquired at the McDonald $2.7 \mathrm{~m}$ in 2009 August, separated by 18 days, and they showed no $\mathrm{RV}$ variation of more than $500 \mathrm{~m} \mathrm{~s}^{-1}$. The measured $T_{\text {eff }}$ and $\log g$ values agreed, within errors, with the KIC values. The low projected rotational velocity of $v \sin i=4 \mathrm{~km} \mathrm{~s}^{-1}$ made this a good target for follow-up with precise RVs. The template spectrum from Keck-HIRES was used to determine the stellar parameters with SME, which was used as inputs to the asteroseismology analysis. The final stellar parameters are $T_{\text {eff }}=5952 \pm 75 \mathrm{~K}, \log g=4.21 \pm 0.04$, and $[\mathrm{Fe} / \mathrm{H}]=$ $-0.08 \pm 0.1$ (see Table 1). The stellar parameters from the KIC, recon spectra, and SME/asteroseismology are all in agreement, 
and no large modifications to the stellar radius nor to the planet radii were needed.

In 2009 September AO imaging at Mt. Palomar revealed two stellar companions in the $J$ band, located 2 ".03 and 5".27 away from the primary target star, with delta magnitudes of 7.4 and 8.1, respectively. Speckle imaging was acquired in 2010 June, and no additional companions were found. The two companions found with AO are beyond the detection limits of speckle. Centroid analysis of the Kepler photometry excludes the possibility that the transits fall on either of the known companion stars. Figure 15 (top left) shows a seeing-limited image of the field of view of the HIRES guide camera that reaches to $\mathrm{Vmag}=21$. It shows four neighboring stars, the two brightest being those seen in the Palomar AO imaging. More stringent limits on companions were placed with Keck AO images we obtained on 2013 June 13/14 and 14/15 yielding a FWHM of 0 '.05 and a detection threshold of $8 \mathrm{mag}$ at $K^{\prime}$ beyond $0^{\prime \prime} .4$ from the primary. The absence of neighboring stars between $0^{\prime \prime} .1$ and $2^{\prime \prime} .0$ reduces the probability of any background and gravitationally bound stars that might masquerade as the two transiting planets. Figure 15 (middle) shows the threshold magnitudes achieved with each highresolution imaging method.

The sparsely populated $\sim 1100$ days time baseline of $\mathrm{RV}$ measurements has an rms of $7.1 \mathrm{~m} \mathrm{~s}^{-1}$ (see Figure 16, top), and the RVs do not correlate in phase with either transiting planet (see Figure 16, bottom right). We believe that the $7.1 \mathrm{~m} \mathrm{~s}^{-1}$ scatter may be due to a combination of the noise, stellar jitter, instrumental errors, and perhaps non-transiting planets not identified with our limited number of RVs.

We provide mass limits from our usual MCMC analysis for each planet. The nominal planet masses are $1.3 \pm 5.4 M_{\oplus}$ and $2.2 \pm 7.8 M_{\oplus}$ for the inner and outer planets respectively. The corresponding density upper limits of $0.3 \pm 2 \mathrm{~g} \mathrm{~cm}^{-3}$ and $0.6 \pm$ $2.3 \mathrm{~g} \mathrm{~cm}^{-3}$ suggest that compositions of pure rocky material are very unlikely in both cases, as the densities are inconsistent with rocky interiors. If they were mostly rocky, both planets would have masses easily detectable by the existing RVs. The full set of RVs are listed, along with their $\log R_{\mathrm{HK}}^{\prime}$ activity values in Table 4.

\section{9. $\mathrm{KOI}-148$, Kepler- 48}

This four planet system, has three transiting planets with orbital periods of $4.8,9.7$, and 43 days with radii of 1.9, 2.7 and $2.0 R_{\oplus}$, respectively. The RVs reveal a non-transiting planet with an orbital period of $982 \pm 10$ days which we name Kepler-48e. It has a minimum mass of $M \sin i=2.1 \pm 0.08 M_{\mathrm{JUP}}$. Follow-up observations of this system began with the acquisition of three recon spectra from the Lick $3 \mathrm{~m}$ and McDonald $2.7 \mathrm{~m}$. The spectral analysis of these recon spectra conducted with SPC found this star to be a slowly rotating main sequence $\mathrm{K} 0$ star, confirming the KIC parameters and adding a measurement of $v \sin i<2 \mathrm{~km} \mathrm{~s}^{-1}$. When we started collecting precise RVs with Keck-HIRES, SME analysis was conducted on the template spectrum. When combined with Yonsei-Yale stellar models, SME found $T_{\text {eff }}=5194 \pm 73 \mathrm{~K}, \log g=4.49 \pm 0.05$ and $[\mathrm{Fe} / \mathrm{H}]=0.17 \pm 0.07$. Final stellar parameters are listed in Table 1.

High-resolution imaging at WIYN using the speckle camera found no stellar companions. AO imaging at Mt. Palomar probed the field of view beyond the $2^{\prime \prime}$ field of view of speckle imaging, revealing four companions within $6^{\prime \prime}$ of the primary. Their separations and brightnesses are: 2 ".44 and delta magnitude of
$4.9,4$ ".32 away and delta magnitude of $3.3,4$.'39 and delta magnitude 7.3, and 5".89 away delta magnitude of 7.0. This Palomar imaging was conducted in the $J$ band and probes down to a $K p$ of 22.1 (Adams et al. 2012). All four of these companion stars are identifiable in the Keck guide camera and care was taken to keep these stars from entering the slit during precise RV observations. However line bisector variations of a few observations suggest that the nearest companion could have been present along the slit on three occasions. Strong limits on stellar companions were imposed with Keck AO imaging on 2013 June 13/14 and 14/15 yielding a FWHM of $0^{\prime \prime} 05$. No additional companions were seen. The detectability curve from all imaging of Kepler-48 is shown in Figure 17. The key result is a lack of stellar companions within the inner $2^{\prime \prime}$. We remain blind to stellar companions within $\sim 0^{\prime \prime} 1$ where Keck AO becomes increasingly less sensitive.

Centroid analysis of the Kepler images, suggests that the transits occurred on the primary star, and not on any of the four companion stars. The centroid-based exclusion radii are less than 0 .'3 for all three planets, ruling out the four stars as sites of the dimming. Figure 17 (top left) shows a seeinglimited image of the field of view from the Keck guide camera. Figure 17 (middle) shows the detection thresholds from the high-resolution imaging analysis.

We took high-resolution spectra of Kepler-48 with KeckHIRES starting in 2009 August (Figure 18, top). The initial RV epochs in summer 2009 would prove vital for detecting the nontransiting planet in a nearly 3 yr orbit. Further RV measurements will more tightly constrain the orbital parameters of the nontransiting planet, which will likewise improve the measurement of the masses of the transiting planets.

The 4.7 days and 9.7 days planets have been shown to be gravitationally interacting, in a 2:1 mean motion resonance, as measured by their TTVs (Steffen et al. 2013; Wu \& Lithwick 2013; Xie 2013). These studies perform a stability analysis to calculate the possibility of these two inner planets as false positives, leading to a FPP of less than 0.001. This agrees with the low FPP we find here, based on Morton (2012) analysis listed in Section 2.3 and Table 3. The planet with the 43 days period does not gravitationally interact with the inner planets in a way that leads to a detectable TTV signal. The masses of the two interacting planets have been measured with TTVs, but with different stellar parameters and different planet radii, making a direct comparison here difficult.

These planet mass values are constrained with a TTV analysis which results in maximum masses of $17.2 \pm 3.9$ and $10.1 \pm$ $3.5 M_{\oplus}$, for 148.01 and 148.02 , respectively. RV measurements currently find the mass of $3.9 \pm 2.1 M_{\oplus}$ and $14.6 \pm 2.3 M_{\oplus}$. Phase-folded RV curves for each planet are shown in Figure 18 (bottom right). Radial velocities constrain the mass of.01 more tightly than TTV analysis. The mass determined for Kepler-48c is consistent between the two methods within errors. A joint analysis using both TTVs and RVs would likely constrain the masses even more.

After the second observing season, a linear trend in the RVs was detected. After the third year of observing the RV trend turned over and the period of the non-transiting planet was known to within a factor of two. Only after the fourth year of observations and a full orbit of the non-transiting planet had occurred, was the period determined to better than $10 \%$. We now find its period, $P=982 \pm 8$ days and $M \sin i=657 \pm$ $25 M_{\oplus}\left(2.1 \pm 0.1 M_{\mathrm{JUP}}\right)$. As the orbital parameters, especially eccentricity, of the non-transiting planet are further constrained 
with more observations, the masses of the inner planets will be more tightly constrained. The full set of RVs are listed, along with their $\log R_{\mathrm{HK}}^{\prime}$ activity values in Table 4.

\subsection{KOI-153, Kepler-113}

Kepler-113 was identified by Kepler as having two transiting planet candidates with orbital periods of $8.9 \mathrm{~d}$ and $4.7 \mathrm{~d}$ having radii of 2.2 and $1.8 R_{\oplus}$, respectively. Recon-level spectra of this system were taken at the $2.6 \mathrm{~m}$ Nordic Optical Telescope and McDonald $2.7 \mathrm{~m}$ in August and 2009 September. Using SPC, stellar parameters of $T_{\text {eff }}$ and $\log g$ were confirmed to be within the errors of the KIC values, and no RV scatter above $1 \mathrm{~km} \mathrm{~s}^{-1}$ was found. This star was found to be a slowly rotating $\mathrm{K} 3 \mathrm{~V}$ star, making it amenable for precise RVs with KeckHIRES. The main challenge posed by this KOI is its faintness, $K p=13.5$ mag, making high-resolution spectroscopy time consuming: Keck-HIRES exposures of 45 minutes are required to achieve $\mathrm{S} / \mathrm{N}=70$ per pixel. The final stellar parameters, determined with SME and Yonsei-Yale stellar models, yielded a stellar radius $30 \%$ less than the KIC value, causing an equal decrease in the determination of the planet radii. The final stellar parameters are $T_{\text {eff }}=4725 \pm 74 \mathrm{~K}, \log g=4.64 \pm 0.03$ and $[\mathrm{Fe} / \mathrm{H}]=0.05 \pm 0.07$, as listed in Table 1 .

Speckle imaging at the WIYN telescope in 2010 June found no companions. AO imaging taken with ARIES in the 2009 observing season and with Palomar in 2010 found one companion within 6 .'0. It was found in both the $K s$ and $J$ bands at $55^{\prime \prime} 14$ from the primary with delta magnitudes of 8.1 and 8.3 respectively. Seeing with ARIES was only 0.'4, worse than typical conditions, while seeing with Palomar was $0.1-00^{\prime \prime} 2$ for $K s$ and $J$ respectively.

Greater sensitivity to stellar companions was achieved with Keck AO imaging, in $K^{\prime}$ bandpass, on 2013 June 14/15 yielding a PSF FWHM of $0{ }^{\prime} .05$, and again on 2013 July 5. The image appears single in the Keck AO image, except for the well known stellar companion located 5".14 from the primary star. This stellar neighbor cannot be the cause of the dimmings because the astrometric exclusion distances are only 0.14 and 0 '.09 for the two planets (see Table 3). Figure 19 at top left shows a seeing-limited image of the field of view of the keck-HIRES guide camera. Figure 19 (middle) shows the limiting magnitudes achieved with each high-resolution imaging method, achieving sensitivity of (and ruling out companions) 7.5 mag fainter than the primary star in near-IR bandpasses.

We obtained precise RVs spanning a time baseline of 832 days (Figure 20, top). The RVs of Kepler-113b ( $P=4.8$ days) phase with the transit-based ephemeris at the 2- $\sigma$ confidence level, yielding a planet mass of $11.7 \pm 4.2 M_{\oplus}$, and a density of $10.7 \pm 3.9 \mathrm{~g} \mathrm{~cm}^{-3}$. The mass and density are consistent with a planet that is composed mostly of rock. The 8.9 day transiting planet is not detected in the RVs and we provide an upper limit to its mass of $9 M_{\oplus}$.

We note that the upper limit of the mass for the 8.9 days planet is difficult to interpret because the peak in the posterior distribution is negative, at $-4.0 M_{\oplus}$. This upper limit is calculated by identifying the 95 th percentile of the posterior mass distribution. The 95th percentile upper limit to mass is thus $9 M_{\oplus}$ and unlikely to be much more massive.

We computed a periodogram of the RV residuals to the best fit. That periodogram exhibits peaks at periods of 1.065 days (strongest), 16 days, 16.4, 0.984 days, and 0.515 days. These periods are aliases of each other. The strongest peak is at 1.065 days and has a false alarm probability near $1 \%$. This false alarm probability is difficult to determine accurately because power is so clearly distributed among the aliases. It appears likely that there is a non-transiting planet with a period likely equal to one of the aliases quoted above. The rms of the RV residuals drops from $8.0 \mathrm{~m} \mathrm{~s}^{-1}$ to $\sim 4.9 \mathrm{~m} \mathrm{~s}^{-1}$ when adopting the 1.065 days period and a circular orbit as a "real" non-transiting planet. The rms decreases less for the other aliases, making them less likely but not ruled out. This reduction in the RV residuals with only three additional parameters (for the circular orbit) indicates that one of the periods is likely real. Further, the lowest value of the reduced $\chi^{2}$ statistic in our fit of the RVs with a three-planet model occurred with the non-transiting planet having $P=1.0651$ days, adding support to that period. A nontransiting planet with $\sim 1$ day period would necessarily be highly misaligned relative to the two transiting planets in the system. Among these four aliases we cannot be sure which is real, if any, and which are the aliases. Thus, we choose not to report any of these periodicities as a definitive "planet candidate." More RV measurements will be needed to assess these periods. The full set of RVs are listed, along with their $\log R_{\mathrm{HK}}^{\prime}$ activity values in Table 4.

\subsection{KOI-244, Kepler-25}

Kepler identified two transiting planets with orbital periods of 12.7 and 6.2 days, with planet radii of 5.2 and $2.7 R_{\oplus}$. The RVs reveal a non-transiting planet with an orbital period of $123 \pm 2$ days and minimum mass of $M \sin i=90 \pm 14 M_{\oplus}$, described below. TTVs for the system were published in Steffen et al. (2012), validating the existence of both planets and providing names, Kepler-25b $(P=6.2$ days $)$ and Kepler-25c $(P=12.7$ days $)$. Note that the names "b" and "c" are placed in order of distance from star, unlike the order of discovery.

Follow-up observations were begun with the acquisition of two recon spectra at the McDonald Observatory $2.7 \mathrm{~m}$ and with the Smithsonian Astrophysical Observatory's Fred L. Whipple Observatory on Mt Hopkins in Arizona, using the Tillinghast Reflector Echelle Spectrograph (TRES), all in 2010 March. The stellar parameters determined from these spectra agree, within errors, with the KIC values. This is a non-typical RV target in the sense that its rotational Doppler broadening is high, $v \sin i=10 \mathrm{~km} \mathrm{~s}^{-1}$, and its surface temperature is high, $T_{\mathrm{eff}}=$ $6270 \mathrm{~K}$, implying few and washed-out absorption lines. The star is rotating faster and has a higher temperature than we normally choose. But its brightness $(K p=10.7)$ makes this a suitable KOI to observe with high spectral resolution. Also, its fast rotation promotes the chance of measuring a Rossiter-McLaughlin (RM) signal. The final stellar parameters, which agreed with the KIC values, were obtained using SME analysis performed on a Keck-HIRES spectrum. SME results were combined with asteroseismology analysis, and the detection of solar like oscillations were used to determine the stellar properties (Huber et al. 2013). The final stellar parameters are $T_{\mathrm{eff}}=6270 \pm 79 \mathrm{~K}$, $\log g=4.28 \pm 0.03$, and $[\mathrm{Fe} / \mathrm{H}]=-0.04 \pm 0.10$, which are listed in Table 1.

Follow-up speckle and AO imaging of Kepler-25 conducted at the WIYN telescope and at the Mt. Palomar $5 \mathrm{~m}$ found no companions, within limits, between $1^{\prime \prime}$ to $6^{\prime \prime}$ from the target. The detection thresholds from Palomar are extraordinary, $7.5 / 8 \mathrm{mag}(\mathrm{J} / \mathrm{K})$ at a separation of $1^{\prime \prime}$ and $>9 \mathrm{mag}$ for $2^{\prime \prime}$ and beyond at $J$ and $K$ bands. Keck AO imaging on 2013 June 13/14 and $14 / 15$ was characterized by a PSF FWHM of 0.05 , offering tight limits on any companions (none found) inward of 0'.5. There was early discussion on the CFOP about a possible false 
positive seen as centroid motion of the primary star in and out of transit. But further analysis ruled out any such displacements to within 2".0, corresponding to half a Kepler pixel. The confusion was due to this star saturating the Kepler CCD light detector. Figure 21 (top) shows a seeing-limited image of the field of view of the HIRES guide camera, with Kepler-25 being the brighter of the two bright stars in that image. Figure 21 (middle) shows the limiting magnitudes achieved with each high-resolution imaging method, with a full detectability curve given in Figure 21. No additional companions were seen.

Gravitational interactions between the two inner planets has been revealed by TTVs as described by Steffen et al. (2012) and Lithwick et al. (2012), and Wu \& Lithwick (2013). Steffen et al. (2012) performs a dynamical analysis of the planet motions based on the observed times of transits, serving as a tool for false-positive assessment. The FPP from the dynamical analysis is $10^{-3}$. Here, employing the extraordinary highresolution speckle and AO imaging, we find a FPP of 0.0001 (see Section 6), lower than from the TTVs alone. Thus, we support the reality of Kepler-25b. The masses are also constrained by TTVs to a similar level of accuracy (Lithwick et al. 2012; Wu \& Lithwick 2013).

Extensive precise RV follow-up was carried out at KeckHIRES from 2009 to 2012 (see Figure 22, top), including two separate measurements of the RM effect, in which RVs were collected continuously while the planet was transiting the host star. The RM results are summarized in Albrecht et al. (2013) showing the stellar spin axis to be well aligned with the orbital axis. Albrecht et al. (2013) find lambda, the projected angle between the orbital plane of the transiting planet and the stellar equatorial plane to be $2^{\circ} \pm 5^{\circ}$. The RVs taken while the planet was transiting were removed for the orbital analysis presented here.

The two transiting planets in the system were confirmed with TTVs, providing upper limits on the planet masses (Lithwick et al. 2012; Wu \& Lithwick 2013). A self-consistent two-planet model shows the RVs to phase well with the transit ephemerides of both planets. For Kepler-25c ( $P=12.7$ days), we find a mass $=24.6 \pm 5.7 M_{\oplus}$, with a corresponding density of $0.90 \pm$ $0.21 \mathrm{~g} \mathrm{~cm}^{-3}$. For Kepler-25b ( $P=6.2$ days $)$ we find a planet mass of $9.6 \pm 4.2 M_{\oplus}$ and a density of $2.5 \pm 1.1 \mathrm{~g} \mathrm{~cm}^{-3}$. The RVs reveal an additional non-transiting planet with of period $123 \pm 2 \mathrm{~d}$, with a lower limit to its mass of $M \sin i=$ $89.9 \pm 13.7 M_{\oplus}$. The orbit of the non-transiting planet is slightly eccentric $(e=0.18)$. The densities of the two transiting planets are both so low that they must consist of some light material, presumably $\mathrm{H}$ and $\mathrm{He}$ (Batygin \& Stevenson 2013).

Spectra of Kepler-25 were also obtained with SOPHIE, the spectrograph dedicated to high-precision RV measurements at the $1.93 \mathrm{~m}$ telescope of the Haute-Provence Observatory, France (Bouchy et al. 2009). SOPHIE is a cross-dispersed, environmentally stabilized echelle spectrograph fed by a set of two optical fibers and calibrated in wavelength with thoriumargon lamps. Observations were secured in high-resolution mode (resolving power $\lambda / \Delta \lambda=75,000$ ). Twelve spectra were secured in 2011 August-November, with exposure times between 30 and 60 minutes allowing $\mathrm{S} / \mathrm{Ns}$ per pixel at $550 \mathrm{~nm}$ between 30 and 70 to be reached. The spectra were extracted using the SOPHIE pipeline, and the RVs were measured from the weighted cross-correlation with a numerical mask characteristic of a G2 star (Baranne et al. 1996), together with the bisector of the cross-correlation function. All the spectral orders except the first 17 were used in the cross-correlation to reduce the dispersion of the measurements. The blue part of the spectra are particularly noisy and using them degrades the accuracy of the RV measurement. The error bars on the RVs were computed from the cross-correlation using the method presented by Boisse et al. (2010); they are typically of the order of $\pm 15 \mathrm{~m} \mathrm{~s}^{-1}$. Three spectra were contaminated by moonlight. Following the method described in Hébrard et al. (2008) we estimated and corrected for the moonlight contamination by using the second SOPHIE fiber aperture, which is targeted on the sky while the first aperture points toward the target. This typically results in RV corrections of a few tens of $\mathrm{m} \mathrm{s}^{-1}$. The final SOPHIE measurements are reported in Table 4 .

The self-consistent three-planet model included RVs obtained with SOPHIE, together with the HIRES RVs and Kepler photometry, to provide the final planet parameters for Kepler$25 \mathrm{~b}$, c, and d, given in Table 2. All parameters for the two transiting planets agree at better than $1 \sigma$ with those obtained above without SOPHIE. This is not surprising as the SOPHIE RV measurements here are about four times less accurate than the HIRES ones. SOPHIE data alone do not allow the two transiting planets to be significantly detected. For the outer, non-transiting planet Kepler-25d, the SOPHIE RVs alone favor an orbital period of $93 \pm 2$ days. That value is an alias with a sampling of $1 \mathrm{yr}$ of the 123 day period reported above for Kepler-25d. Additional observations on a longer time span will allow the correct orbital period to be established. This will be the object of a forthcoming paper. Whether the period for Kepler$25 \mathrm{~d}$ is 93 or 123 days has no significant effect on the derived semi-amplitudes or masses measured for each of the three planet masses for Kepler-25.

Finally, we studied the variations of the line bisectors obtained with HIRES and SOPHIE. We found neither variations nor trends as a function of RV. The RV residuals considering only one or two of the detected planets do not show either any correlations with the bisectors. This reinforces the conclusion that each of the three RV variations are due to planetary signals, and not caused by spectral-line profile changes attributable to blends or stellar activity. The full set of RVs, minus the RVs used to measure the RM effect, are listed, along with their $\log R_{\mathrm{HK}}^{\prime}$ activity values in Table 4 .

\subsection{KOI-245, Kepler-37}

Kepler identified three transiting planets having orbital periods of $13.36,21.30$, and $39.79 \mathrm{~d}$ with corresponding planet radii of $0.32,0.75$ and $1.94 R_{\oplus}$, respectively. A fourth candidate transiting planet was reported in Batalha et al. (2013), but it has since been deemed a false positive and it is not included in this study. A detailed analysis of the Kepler light curve, blend scenarios, and asteroseismic analysis of this exceptionally bright $(K p=9.7)$ KOI with a sub-Mercury sized planet can be found in Barclay et al. (2013). The existences of all three planets were validated, yielding new names, Kepler-37 b,c,d. Here, we summarize the follow-up observations, and we place limits on the planet masses from the precise RV observations.

Recon spectra from the McDonald $2.7 \mathrm{~m}$ and the Tillinghast $1.5 \mathrm{~m}$ were taken on 2010 March and 2010 April, respectively. These spectra confirmed the stellar parameters from the KIC, and showed that $v \sin i$ is less than $2.0 \mathrm{~km} \mathrm{~s}^{-1}$. SME analysis of a Keck-HIRES spectrum also agreed with the KIC parameters, and the final stellar parameters are listed in Table 1, which were determined via asteroseismology, with SME-based $T_{\text {eff }}, \log g$, and $[\mathrm{Fe} / \mathrm{H}]$ used as inputs. The final stellar parameters are $T_{\text {eff }}=$ $5417 \pm 75 \mathrm{~K}, \log g=4.57 \pm 0.05$, and $[\mathrm{Fe} / \mathrm{H}]=-0.32 \pm 0.07$, 
and are consistent with those in Barclay et al. (2013). This KOI is the most dense star yet to reveal asteroseismic oscillations, made possible by its brightness and lengthy coverage in short cadence observations.

We present a seeing-limited image of the field of view in Figure 23 (top left). There is a stellar neighbor located 7 " south of the primary star and about 4 mag fainter in $V$ band. Speckle imaging was acquired at the WIYN telescope and also at the Gemini Telescope, showing Kepler-37 to be a single star within 2 ".7 square box, with no companion detected to a threshold of 6 mag in $R$ band and $5.1 \mathrm{mag}$ in $V$ band. No neighboring star was seen. Near infrared AO taken with ARIES also revealed no stellar companions. Extensive AO observations were also taken with the Mt. Palomar $5 \mathrm{~m}$ telescope using a Brackett-gamma filter to avoid saturation of the IR detector. No stellar companion was found. A more thorough analysis of these AO observations is found in Barclay et al. (2013) where the probability of background stars falling, undetected, into the Kepler aperture, is discussed. Still we obtained Keck AO images on 2013 June $14 / 15$ to achieve even better imaging of the region $0.1-0^{\prime \prime} .5$ from the star. The detectability curves from Keck AO and from all imaging are shown in Figure 15, showing detectability at near-IR wavelengths 8 mag fainter for all angular separations beyond 0.3 . No stellar companion was seen.

The full set of RVs are listed, along with their $\log R_{\mathrm{HK}}^{\prime}$ activity values in Table 4. The precise RVs from Keck-HIRES are unable to determine the masses of the three transiting planets because their expected RV amplitudes are all below $1 \mathrm{~m} \mathrm{~s}^{-1}$. We constrain the mass of the 40,21, and 13 day planets to be less than 12.2, 12.0, and $10.0 M_{\oplus}$, respectively, based on the 95th percentile positive extent of the posterior distribution of planet mass. The mass for the 40 days planet corresponds to a density upper limit of $8.7 \mathrm{~g} \mathrm{~cm}^{-3}$, not useful to distinguish pure rocky from mostly volatile-rich compositions. The 21 and 13 day planets similarly do not offer useful constraints on their densities. The 862 day baseline for the RV measurements finds no significant periods or trends above the noise. Thus no non-transiting planets are detected.

\subsection{KOI-246, Kepler-68}

Kepler identified two transiting planets with orbital periods of 5.40 and 9.60 days and planet radii of 2.3 and $0.95 R_{\oplus}$, as described and validated in Gilliland et al. (2013), giving them names, Kepler-68 b and c. Our RVs indicate a non-transiting planet with an orbital period of $625 \pm 16$ days and mass of $267 \pm 16 M_{\oplus}$. Here, the planet properties and orbital parameters are refined over those provided in Gilliland et al. (2013), with five additional RVs obtained in 2013. For a detailed summary of the light curve analysis, asteroseismic analysis, RV analysis, and discussion of false-positive scenarios with BLENDER, see Gilliland et al. (2013). Recon spectra were taken at the McDonald $2.7 \mathrm{~m}$ on 2010 March 25 and 2010 March 28. A spectrum was acquired at Tillinghast $1.5 \mathrm{~m}$ on 2010 March 25 . The near solar values of temperature, $\log g$ and $v \sin i$ listed in the KIC were confirmed by these recon spectra. SME combined with asteroseismology analysis was then used to confirm the near solar values of stellar mass and stellar radius. The final stellar parameters are $T_{\text {eff }}=5793 \pm 74 \mathrm{~K}, \log g=4.28 \pm 0.02$, and $[\mathrm{Fe} / \mathrm{H}]=0.12 \pm 0.07$, listed in Table 1 .

Speckle imaging at WIYN taken in 2010 June and AO imaging taken with ARIES, in summer 2010 found no companion stars that could cause confusion or pollution in the light curve analysis. Figure 25 (top left) shows a seeing-limited image of the field of view of the HIRES guide camera. Figure 25 (middle) shows the detection threshold delta magnitudes achieved with each high-resolution imaging method. While Gilliland had already effectively ruled out false positives, we obtained Keck AO images on 2013 June 14/15 yielding a FWHM of 0.'05, with a full detectability curve given in Figure 25, supporting and improving the lack of stellar companions to within $0{ }^{\prime \prime} 05$ of the star.

The precise RVs collected from 2009 to 2013 (see Figure 26, top) determine the mass and density of the $2.3 R_{\oplus}$, innermost transiting planet to be $5.97 M_{\oplus}$ and $2.60 \pm 0.74 \mathrm{~g} \mathrm{~cm}^{-3}$, respectively. The RVs also provide upper limits to the mass of the second transiting planet. All current planet parameters for Kepler-68 are listed in Table 2), and they differ from those in Gilliland et al. by typically $\sim 1 \sigma$.

The RVs clearly reveal a non-transiting planet with an orbital period of $625 \pm 16$ days, with a minimum mass $(M \sin i)$ of $0.84 \pm 0.06 M_{\mathrm{JUP}}$. During the initial publication of the Kepler-68 results, the period of the non-transiting planet had a high eccentricity alias solution (I. Crossfield 2013, private communication). The RVs obtained in 2013 show the true period to be that quoted both here and in Gilliland et al. (2013), not the alias. The full set of RVs are listed, along with their $\log R_{\mathrm{HK}}^{\prime}$ activity values in Table 4 .

The innermost planet with its density of $2.6 \mathrm{~g} \mathrm{~cm}^{-3}$ occupies an important niche in exoplanet science. This density is intermediate between that of purely rocky planets and the mostly gaseous ones. Lopez et al. (2012), Lopez \& Fortney (2013) discusses the implications of such hybrid planets.

\subsection{KOI-261, Kepler-96}

The Kepler photometry revealed a single transiting planet in this system with a period of $16.2 \mathrm{~d}$ and a radius of $2.67 R_{\oplus}$. Three recon spectra were taken at the McDonald $2.7 \mathrm{~m}$ and Tillinghast Observatories in 2010 March and 2010 April. Stellar parameters determined from these spectra with SPC agreed in $T_{\text {eff }}$, but the $\log g$ value was off by 0.4 dex from the KIC value. When the new $\log g$ was confirmed with SME analysis of a Keck-HIRES template spectrum, Yonsei-Yale stellar models were used to adjust the stellar radius from its KIC value of 1.9 to $1.02 \pm 0.09 R_{\odot}$. The planet radius decreased from 6.2 to $2.7 R_{\oplus}$. Further evidence that the stellar radius is near solar, and not near $1.9 R_{\odot}$, is the non-detection of stellar oscillations in the asteroseismic analysis. A typical $1.9 R_{\odot}$ star would have a detectable asteroseismic signal, which was searched for and not detected. The final stellar parameters are $T_{\text {eff }}=5690 \pm 73 \mathrm{~K}$, $\log g=4.42 \pm 0.08$, and $[\mathrm{Fe} / \mathrm{H}]=0.04 \pm 0.07$ and other stellar values are listed in Table 1 .

High-resolution imaging with the WIYN speckle camera in 2010 September detected no stellar companions, but MMTARIES observations in summer 2010 detected one nearby companion located 5..4 northeast of the primary star at P.A. $=65^{\circ} .2$, and delta magnitude of 7.1 in $J$ band, and 6.8 in $K s$ band. The estimated Kepler magnitude of the companion is 18.1 . Figure 27 (at top left) shows a seeing-limited image of the field of view of the HIRES guide camera. Figure 27 (middle) shows the detection threshold magnitudes achieved with each high-resolution imaging method. A detailed analysis of the rotation period (from the Kepler photometry) and the rotational Doppler broadening, $v \sin i$, indicates the star may be oriented nearly pole-on, suggesting a spin-orbit misalignment (Hirano et al. 2012). Tighter limits on stellar companions were placed with Keck AO imaging on 2013 June 13/14, yielding a 
resolution of 0.05 (FWHM), and all of the detectability curves from all imaging attempts are given in Figure 27. No additional companions were seen besides that located 5.4 away, noted above.

Precise RV follow-up was initiated in 2010 July and 25 RVs have been acquired for a time baseline of 772 days (Figure 28, top). The RVs show a weak correlation with the Kepler transit ephemeris. We constrain the RV $K$ amplitude to be $2.1 \pm$ $0.8 \mathrm{~m} \mathrm{~s}^{-1}$. This translates to a planet mass of $8.46 \pm 3.4 M_{\oplus}$. The corresponding density is $2.26 \pm 1.11 \mathrm{~g} \mathrm{~cm}^{-3}$, which is significant in that we constrain the density to be less than $3.4 \mathrm{~g} \mathrm{~cm}^{-3}$ at the $1 \sigma$ level and $4.5 \mathrm{~g} \mathrm{~cm}^{-3}$ at the $2 \sigma$ level. Such a low density rules out a purely rocky composition, and requires some contribution from low-density materials such as water, $\mathrm{H}$ or He. The RVs show no sign of any nontransiting planet after $3 \mathrm{yr}$ at levels of $\sim 3 \mathrm{~m} \mathrm{~s}^{-1}$. The full set of RVs are listed, along with their $\log R_{\mathrm{HK}}^{\prime}$ activity values in Table 4.

As in the case of Kepler-25, Kepler-96 was observed with SOPHIE. Six 60 minute observations were secured in 2011 July-September, allowing S/Ns of the order of 70 and RV accuracy of $\pm 25 \mathrm{~m} \mathrm{~s}^{-1}$ reached on each exposure. The SOPHIE RVs do not allow the upper limit on the reflex motion due to Kepler-96b to be significantly improved.

\subsection{KOI-283, Kepler-131}

Kepler identified two transiting planets with orbital periods of 16.0 and 25.5 days and radii 2.4 and $0.8 R_{\oplus}$. The two recon spectra taken with the McDonald $2.7 \mathrm{~m}$ and the TRES spectrometer gave good agreement in $T_{\text {eff }}$ but the value of $\log g$ found with spectroscopy is 0.5 dex larger than the KIC value, resulting in a decrease in stellar radius of $40 \%$. An SME analysis of a Keck-HIRES spectrum combined with Yonsei-Yale stellar models gave $T_{\text {eff }}=5685 \pm 74 \mathrm{~K}, \log g=4.42 \pm 0.08$ and $[\mathrm{Fe} / \mathrm{H}]=0.12 \pm 0.07$, listed in Table 1 .

Speckle imaging from the WIYN found no stellar companions and the lone stellar companion detected with AO imaging from Mt. Palomar is 6" away and 8 mag fainter in $J$ band and $K s$ band. Such a stellar neighbor could not be responsible for the dimming, as it would be easily detected with astrometric centroid motion analysis in and out of transit in the Kepler images. Keck AO imaging took place on 2013 June 13/14, yielding a FWHM of 0 .'05, with a full detectability curve given in Figure 15 , revealing no additional companions. Figure 29 (top left) shows a seeinglimited image of the field of view of the HIRES guide camera. Figure 29 (middle) shows the limiting magnitudes achieved with each high-resolution imaging method.

The RV measurements for Kepler-131 span 741 days (Figure 30, top) and are listed, along with their $\log R_{\mathrm{HK}}^{\prime}$ activity values in Table 4 . The results consist of an RV detection of the $16 \mathrm{~d}$ planet giving a mass of $16.1 \pm 3.5 M_{\oplus}$, and a density of $6.0 \pm 2.0 \mathrm{~g} \mathrm{~cm}^{-3}$. We find a similar mass and density whether we fit this planet by itself or with the second planet simultaneously. The second planet shows up in the RVs only marginally. A self-consistent fit of RVs and photometry yields a peak of the posterior mass distribution at $M=8.25 \pm 5.9 M_{\oplus}$ and density of $78 \pm 55 \mathrm{~g} \mathrm{~cm}^{-3}$. The density is unphysically large, and hence the mass is too large, indeed a detection at less than $2 \sigma$. We find an upper limit to the mass of Kepler-131c from the 95th percentile of the posterior mass distribution, yielding $M<20.0 M_{\oplus}$. Certainly the mass of Kepler-131c remains highly uncertain. Table 2 lists all of the best-fit planet parameters.

\subsection{KOI-292, Kepler-97}

The single transiting planet identified by Kepler in this system has an orbital period of $2.587 \mathrm{~d}$ and a radius of $1.5 R_{\oplus}$. Followup observations began with recon spectra in 2010 March and 2010 April at the McDonald $2.7 \mathrm{~m}$ telescope. SPC analysis found this star to be a slowly rotating main sequence star, an ideal target for Keck-HIRES spectroscopy, the analysis of which using Yonsei-Yale stellar models gave a final stellar radius $30 \%$ smaller than that reported in the KIC. The final stellar parameters are $T_{\text {eff }}=5779 \pm 74 \mathrm{~K}, \log g=4.43 \pm 0.08$, and $[\mathrm{Fe} / \mathrm{H}]=$ $-0.20 \pm 0.07$, sub-solar metallicity, with all stellar parameters in Table 1

AO imaging at both Palomar and Keck-NIRC2 shows a companion star with an angular separation of $0.37 \pm 0.01$ from the primary at a P.A. $=121.8$. The companion was measured to be $2.7 \mathrm{mag}$ fainter in the optical (from speckle observations), $2.8 \mathrm{mag}$ fainter than the primary in the $J$ band, and $3.2 \mathrm{mag}$ fainter in $K^{\prime}$ band. These magnitude differences have uncertainties of $0.15 \mathrm{mag}$. The Keck AO images revealed no additional neighboring stars (besides the one 0'.37 away), down to delta magnitude of $8 \mathrm{mag}$ ( $K^{\prime}$ band), shown in detail in Figure 31. Speckle imaging revealed no companions down to a delta magnitude of 3 and 4 in the $R$ band and $V$ band, respectively. The non-detection with speckle is likely due to the companion being brighter in the infrared than in the visible, relative to the primary. The companion is listed in Adams et al. (2012), and makes this a less than ideal target for precise RVs due to the contamination of the companion in the spectrum of the primary. Figure 31 (top left) shows a seeing-limited image of the field of view of the HIRES guide camera. Figure 31 (middle) shows the limiting magnitudes achieved with each high-resolution imaging method.

The stellar companion located 0 '.37 from the primary star resides farther than the maximum exclusion radius determined from the astrometric centroid measurements in and out of transit. Thus, it is unlikely that the neighboring star is responsible for the dimmings. If it were, the centroid of light would appear to shift more than the upper limit exclusion radius (see Table 3). But it remains difficult to calculate the possibility that the centroid measurements are not robust enough to rule out this stellar companion. We remain concerned that while formally this stellar companion is ruled out as the cause of the apparent transits, this assessment should be revisited.

The full set of RVs are listed, along with their $\log R_{\mathrm{HK}}^{\prime}$ activity values in Table 4 . The RVs exhibit a weak downward trend of $5 \mathrm{~m} \mathrm{~s}^{-1}$ per year during three seasons ( $2 \mathrm{yr}$ ). Figure 32 shows no deviations from a monotonic downward trend, but more RVs are needed to confirm this trend. The companion causing the linear trend likely has a an orbital period longer than the twice the time baseline, $P>4 \mathrm{yr}$ and a mass greater than $1 M_{\mathrm{JUP}}$. The RV trend may be caused by the stellar companion 0.37 away found with the AO imaging, but we have not pursued this question in detail.

Fitting for the linear trend, the RVs marginally correlate with the ephemeris of the transiting planet. The best-fit planet mass is $M=3.51 \pm 1.9 M_{\oplus}$, corresponding to a density of 5.44 $\pm 3.48 \mathrm{~g} \mathrm{~cm}^{-3}$. This is less than a $2 \sigma$ detection of mass and density, meriting more RV measurements. We adopt an upper limit on the mass of the transiting planet to be $9.1 M_{\oplus}$ from the 95th percentile of the posterior mass distribution. This upper limit corresponds to an upper limit for the planet density of $14 \mathrm{~g} \mathrm{~cm}^{-3}$, which seems unphysically high. Table 2) gives all 
of the resulting planet parameters. The phase-folded RV curves are shown in Figure 32 (bottom right).

\subsection{KOI-299, Kepler-98}

Kepler identified a transiting planet around Kepler-98 with an orbital period of $1.5 \mathrm{~d}$ and a radius of $2.0 R_{\oplus}$. Before precise RVs were acquired, recon spectra were taken with the McDonald $2.7 \mathrm{~m}$ in 2010 March and the Tillinghast $1.5 \mathrm{~m}$ (TRES spectrometer) in 2010 June. The stellar surface temperatures found by SPC from the recon spectra were in fair agreement with KIC values. We acquired a spectrum with Keck-HIRES, giving final stellar parameters of $T_{\text {eff }}=5589 \pm 73 \mathrm{~K}, \log g=4.34 \pm$ 0.10 and $[\mathrm{Fe} / \mathrm{H}]=0.18 \pm 0.07$, found by SME analysis and matching of spectral parameters to Yonsei-Yale stellar models (see Table 1).

Speckle imaging at the WIYN telescope conducted in 2010 September detected no companions within its limits. Keck AO imaging took place on 2013 June 14, yielding a FWHM of 0'.05, with a full detectability curve given in Figure 33. No stellar companions were detected at limits of 8 magnitues fainter (in $K$ band) than the primary star. The Keck guider image (Figure 33, top left) shows no companions from $1^{\prime \prime}$ to $6^{\prime \prime}$ from the primary, down to a delta magnitude of 7 .

The full set of RVs are listed, along with their $\log R_{\mathrm{HK}}^{\prime}$ activity values in Table 4 . The RVs cover a time baseline of $2 \mathrm{yr}$ and show only a marginal detection of the transiting planet (Figure 34). We find its mass to be $3.55 \pm 1.6 M_{\oplus}$ with a density of $2.2 \pm$ $1.2 \mathrm{~g} \mathrm{~cm}^{-3}$. The 95 th percentile of the posterior mass distribution provides a useful upper limit to the planet mass of $6.4 M_{\oplus}$ and a corresponding upper limit to density of $3.9 \mathrm{~g} \mathrm{~cm}^{-3}$. This upper limit to the density of the planet indicates a likely composition consistent with significant contributions of low-density material, presumably $\mathrm{H}, \mathrm{He}$, and/or water. Table 2 gives the full set of planet parameters.

\subsection{KOI-305, Kepler-99}

The single transiting planet found by Kepler in this system has an orbital period of $4.6 \mathrm{~d}$ and a radius of $1.5 R_{\oplus}$. Recon spectroscopy of the target was initiated in 2010 March at the McDonald $2.7 \mathrm{~m}$. In 2010 May, a second recon spectrum was taken at the Tillinghast $1.5 \mathrm{~m}$. Both spectra were used to determine $T_{\text {eff }}$ and $\log g$. The stellar parameters determined from each spectrum were self-consistent. These were in agreement with the KIC values in $T_{\text {eff }}$, but only in marginal agreement with the KIC in $\log g$. No large (over $1 \mathrm{~km} \mathrm{~s}^{-1}$ ) RV variation was seen and the low rotational velocity of the star led to collection of precise RVs. Once a Keck-HIRES template was taken, SME was used with Yonsei-Yale stellar models to determine the final stellar parameters of $T_{\text {eff }}=4782 \pm 129 \mathrm{~K}, \log g=4.61 \pm 0.05$, and $[\mathrm{Fe} / \mathrm{H}]=0.18 \pm 0.07$. The final stellar radius is $30 \%$ smaller than the KIC values (see Table 1).

Speckle imaging taken at the WIYN telescope found no companions within its limits and no further imaging is available. Keck AO imaging took place on 2013 June 15, yielding a FWHM of 0 '. 05 , with a full detectability curve given in Figure 35, revealing no companions. When observing with Keck-HIRES, a guider image was taken. No companions were detected from $2^{\prime \prime} 0$ out to $4^{\prime \prime} 0$ within seven magnitudes of the primary star in the $R$ band. Figure 35 (top left) shows a seeinglimited image of the field of view of the HIRES guide camera.

The full set of RVs are listed, along with their $\log R_{\mathrm{HK}}^{\prime}$ activity values in Table 4 . The RV baseline of 791 days (Figure 35, top) shows no linear trends or periodicities aside from the transiting planet. This single, $1.5 R_{\oplus}$ planet has a mass determined from RVs of $6.15 \pm 1.3 M_{\oplus}$. The planet density is $10.9 \pm 2.8 \mathrm{~g} \mathrm{~cm}^{-3}$, suggesting the planet has a rocky interior, similar in density to Kepler-10b and CoRoT-7b (see Kepler-406 below). The phasefolded RV curve is shown in Figure 36 (bottom right).

\subsection{KOI-321, Kepler-406}

The Kepler mission identified two transiting planets around Kepler-406, having orbital periods of 2.43 and 4.62 days with radii of 1.43 and $0.85 R_{\oplus}$, respectively. Recon spectroscopy was taken at the Tillinghast $1.5 \mathrm{~m}$ (TRES), where $T_{\text {eff }}$ was confirmed to be near the KIC value. The slow rotational broadening of the stellar lines was seen $\left(v \sin i<3 \mathrm{~km} \mathrm{~s}^{-1}\right)$, and any RV variation was clearly below $500 \mathrm{~m} \mathrm{~s}^{-1}$. The $\log g$ value found by recon, and later refined with SME and asteroseismology analysis, is larger than that in the KIC, leading to an increase in the stellar and planet radii of $30 \%$. The final stellar parameters, although not published in Huber et al. (2013), were found using similar methods. The SME analysis of the Keck-HIRES spectrum served as the starting point of the asteroseismology analysis that led to $T_{\text {eff }}=5538 \pm 44 \mathrm{~K}, \log g=4.41 \pm 0.02$, and $[\mathrm{Fe} / \mathrm{H}]=0.18 \pm 0.04$. Table 1 contains the full set of stellar parameters including mass and radius.

Imaging including Speckle imaging with WIYN in 2010 September and AO imaging taken with the Lick 3m in 2011 September, found no companions nearby that are contaminating the light curve. Keck AO imaging took place on 2013 June 13, yielding a FWHM of 0 '. $^{\prime} 05$, with a full detectability curve given in Figure 37, also revealing no neighboring stars. Figure 37 (top left) shows a seeing-limited image of the field of view of the HIRES guide camera.

The full set of RVs are listed, along with their $\log R_{\mathrm{HK}}^{\prime}$ activity values in Table 4. Precise RVs taken over 800 days, beginning in 2010 July, are shown in Figure 38, top. The inner transiting planet appears clearly in the RVs phased to the ephemeris from the transits. We find this $1.43 R_{\oplus}$ planet to have a mass of $6.35 \pm$ $1.4 M_{\oplus}$ with a corresponding density of $11.8 \pm 2.7 \mathrm{~g} \mathrm{~cm}^{-3}$. Such high density, measured at the $4 \sigma$ confidence level, implies that this planet is essentially made of rock and iron/nickel, perhaps compressed. This planet represents one of the most convincing cases of a rocky planet around a main sequence star, joining CoRoT-7b, Kepler-10, and Kepler-36b.

The outer transiting planet shows only a marginal signal in the RVs, yielding a mass of $2.71 \pm 1.8 M_{\oplus}$ and a density of $24.4 \pm$ $16 \mathrm{~g} \mathrm{~cm}^{-3}$. This density is unphysically high, indicating that the RVs provide only a upper limit. We adopt the 95th percentile of the posterior mass distribution, giving an upper limit to its mass of $6.0 M_{\oplus}$.

\subsection{KOI-1442, Kepler-407}

With a period of 0.67 days and a radius of $1.1 R_{\oplus}$, Kepler-407b has a similar orbital period to the planet Kepler-10b. However, the RVs provide only upper limits to the transiting planet's mass, and a partial orbit of a non-transiting planet. Followup observing began in 2011 March when recon spectra were collected at the McDonald $2.7 \mathrm{~m}$. SPC analysis of the spectrum confirmed the $T_{\text {eff }}$ and $\log g$ from the KIC. After acquisition of the Keck-HIRES template spectrum, SME analysis was run, and the final stellar parameters were calculated by combining the SME result with Yonsei-Yale isochrones. The final stellar parameters are $T_{\text {eff }}=5476 \pm 75 \mathrm{~K}, \log g=4.43 \pm 0.06$ and $[\mathrm{Fe} / \mathrm{H}]=0.04 \pm 0.07$. 
Speckle observing at WIYN in 2010 September did not identify any companion stars within the limits. There is one companion identified in UKIRT images that is outside of the field of view of speckle. The companion star is 2 ." 1 to the NE of the primary and roughly 5.3 mag fainter in the Kepler bandpass. Centroid analysis of the pixel level data constrains the centroid of the planet transit to be within 0'.44 of the primary, ruling out the possibility that the transits are actually occurring on a nearby star. Figure 39 (top left) shows a seeing-limited image of the field of view of the HIRES guide camera. Figure 39 (middle) shows the limiting magnitudes achieved with speckle imaging. Keck AO imaging took place on 2013 June 13/14, yielding a FWHM of 0 '.05, with a full detectability curve given in Figure 39. We found no stellar companions, except for the known companion 2 ". 1 away, thus greatly limiting the FPP, as discussed in Section 6.

The most prominent signal in the RVs is a decrease of $300 \mathrm{~m} \mathrm{~s}^{-1}$ during the $2 \mathrm{yr}$ baseline, with clear curvature, as seen in Figure 40, top. The slight curvature in the RVs is indicative of a massive body in a orbit of roughly 6-12 yr, with a minimum mass, $M \sin i \sim 5-10 M_{\mathrm{JUP}}$. With only roughly one quarter of this potential decade long orbit observed, the outer body remains poorly constrained in both orbital period and $M \sin i$. Its mass could be above $10 M_{\mathrm{JUP}}$ and even above $80 M_{\mathrm{JUP}}$, for extremely face-on orbits. Thus the non-transiting object is likely a planetary body of 5-20 $M_{\mathrm{JUP}}$, but could be classed as a brown dwarf or stellar, depending on the orbital inclination. The RVs in the next few years will settle its $M \sin i$. The transiting planet and the non-transiting body are fit simultaneously. There are too few RV points to confidently measure the mass of the transiting planet and we place a $1 \sigma$ upper limit on the mass of $1.7 \mathrm{M}_{\oplus}$. The phase-folded RV curves are shown in Figure 40 (bottom right). We caution that the transiting planet could be orbiting that massive, non-transiting body, if luminous, rather than the primary star. The full set of RVs are listed, along with their $\log R_{\mathrm{HK}}^{\prime}$ activity values in Table 4 .

\subsection{KOI-1612}

The single planet in this system has a period of 2.5 days and a radius of $0.82 R_{\oplus}$. This F-type star was first followed up from the ground at the Tillinghast $1.5 \mathrm{~m}$ where two spectra were taken. SPC determined the stellar parameters to be consistent with the KIC and showed no dramatic RV variation. Before the asteroseismology analysis was conducted in Huber et al. (2013), Bruntt et al. (2012) identified this bright star as having detectable asteroseismic oscillations. We use the SME-asteroseismology values in this work. The $T_{\text {eff }}=6104 \pm 74 \mathrm{~K}, \log g=4.29 \pm$ 0.03 , and $[\mathrm{Fe} / \mathrm{H}]=-0.20 \pm 0.10$.

Speckle imaging taken at the WIYN in 2011 July found no companion stars within the limits. Figure 41 (top left) shows a seeing-limited image of the field of view of the HIRES guide camera. Figure 41 (middle) shows the limiting magnitudes achieved with speckle imaging. Keck AO imaging took place on 2013 June 13/14, yielding a FWHM of $0^{\prime \prime} .05$, with a full detectability curve given in Figure 41. No stellar companions were found. As described in Section 6, this stringent AO nondetection from Keck was able to limit the FPP to $2.1 \%$, but not below $1 \%$.

The first precise RVs were obtained shortly after the recon spectra were taken in 2011 May, resulting in an RV baseline of 477 days (Figure 42, top). The brightness of the star allowed us to obtain high signal to noise observations $(\mathrm{S} / \mathrm{N}=200)$, similar to those obtained for the standard CPS planet search stars. While we have not directly detected the planet, we place a $1 \sigma$ upper limit on the mass of the planet equal to $4.3 M_{\oplus}$, but the limit on density is too uncertain to provide insight into composition. There are no RV signatures or periodicities that suggest the presence of a non-transiting planet in the system that would predict an RV amplitude greater than $4 \mathrm{~m} \mathrm{~s}^{-1}$. A longer time baseline of observations will further constrain nontransiting planets. The posterior distribution of expected mass values is well behaved, and peaks near zero, as expected for RVs with well understood, photon-limited uncertainties that provide only upper limits on the planet mass. The phase-folded RV curve is shown in Figure 42 (bottom right). The full set of RVs are plotted in Figure 42 (top) and listed, along with their $\log R_{\mathrm{HK}}^{\prime}$ activity values, in Table 4.

\subsection{KOI-1925, Kepler-409}

This KOI with a radius of $1.2 R_{\oplus}$ and period of 69 days was first followed up in the 2012 observing season. Kepler-409 is one of Kepler's brightest KOIs ( $K p=9.44 \mathrm{mag}$ ) with a near earth sized planet. It has well determined parameters from asteroseismology (Bruntt et al. 2012; Huber et al. 2013). This analysis determined that the final value of $\log g$ differed from the KIC value and therefore the stellar radius was adjusted to only $40 \%$ of the KIC value. Recon spectra from the Tillinghast $1.5 \mathrm{~m}$ were taken and broadly confirmed the analysis of asteroseismology. The final stellar parameters are $T_{\text {eff }}=5460 \pm 75 \mathrm{~K}, \log g=$ $4.50 \pm 0.03$ and $[\mathrm{Fe} / \mathrm{H}]=0.08 \pm 0.10$.

Careful Keck AO imaging took place on 2013 June 13/14, yielding a FWHM of 0 '.05, with a detectability curve given in Figure 43 . We found no stellar companions, greatly limiting the FPP, as discussed in Section 6.

Keck-HIRES RVs taken over 174 days in summer 2012 (Figure 44, top) all have high $\mathrm{S} / \mathrm{Ns}$ of 200 . This $\mathrm{S} / \mathrm{N}$ is similar to the typical planet search stars, acquired in roughly ten minute exposures. The planet's expected RV amplitude, assuming an approximate mass-radius relationship tied to known low mass planets (Weiss et al. 2013), is below our detection threshold. Nonetheless, we limit the planet's mass to be less than $8.9 M_{\oplus}$ at the $1 \sigma$ level. Such limits are insufficient to make conclusions about planet composition. With $25 \mathrm{RVs}$, showing an rms of only $3.2 \mathrm{~m} \mathrm{~s}^{-1}$ we place upper limits on the mass of the transiting planet, and rule out any massive $\left(M \sin i>30 M_{\oplus}\right.$, expected $K$ amplitude $=4.5 \mathrm{~m} \mathrm{~s}^{-1}$ ) planets in orbits interior to the transiting planet.

The full set of RVs are plotted in Figure 44 (top) and listed, along with their $\log R_{\mathrm{HK}}^{\prime}$ activity values in Table 4 .

\section{DISCUSSION}

\subsection{Science Drivers and Selection Effects}

NASA's Kepler mission has discovered over 4000 planet candidates to date, offering unprecedented statistical information on the occurrence, sizes, and orbits of planets, including over 400 multi-planet systems(Batalha et al. 2013; Fabrycky et al. 2012; Lissauer et al. 2012). Among the remarkable results is that $30 \%-50 \%$ of Sun-like stars harbor planets of size, $1-4 R_{\oplus}$, residing in orbits within 0.5 au (Batalha et al. 2013; Howard et al. 2012; Petigura et al. 2013; Fressin et al. 2013). The small M dwarf stars also commonly harbor such small planets (Dressing \& Charbonneau 2013).

The nature of these $1-4 R_{\oplus}$ planets within 0.5 au remains poorly understood, including their formation and internal composition. They may have formed as imagined for Neptune and 
Uranus, beyond the ice line where they accreted gas and ices, notably $\mathrm{H}$ and $\mathrm{He}$ as well as water. Alternatively, $1-4 R_{\oplus}$ planets may be "terrestrial" planets that formed as did Earth, Venus, and Mars, but also acquired varying, small amounts of $\mathrm{H}$ and $\mathrm{He}$ to puff their sizes above $1 R_{\oplus}$ (Batygin \& Stevenson 2013; Zeng \& Seager 2008; Rogers et al. 2011; Valencia et al. 2007).

The nature of the $1-4 R_{\oplus}$ planets also bears on habitability, as atmospheres of tiny mass yield modest pressures at the surface allowing complex organic molecules to exist (Seager et al. 2013). But $\mathrm{H}$ and $\mathrm{He}$ "atmospheres" having over $1 \%$ the mass of the planet will cause surface pressures above 1 Mbar and temperatures above $1000 \mathrm{~K}$, causing severe intermolecular Van der Waals interactions that threaten the existence of complex organic molecules such as RNA or DNA.

Here, we studied 42 small transiting planets and 7 nontransiting planets that orbit 22 KOIs. This KOI sample was selected from among the candidate transiting planets identified by Kepler (Batalha et al. 2013), as described in Section 2. The selection criteria stemmed from the goal of constraining the interior structure of planets smaller than $\sim 4 R_{\oplus}$, with planets smaller than $2.5 R_{\oplus}$ being higher priority. Measuring small-planet masses with RV measurements required orbital periods short enough that the reflex motion of the star might be detectable by precise Doppler measurements. However, we had no prior knowledge of the planet masses in advance, and thus there was no selection effect in mass, for a given planet radius. Planets having photometrically determined radii could have had any mass prior to our RV measurements.

With extensive observations and modeling of false-positive channels, we found an FPP under $1 \%$ for all of the transiting planets except two which have only a $\sim 2 \%$ FPP (see Section 6 and Table 3). The likely existence of all 42 transiting planets allows them to be employed in statistical studies, notably of their radii, masses, densities, orbits, and host stars.

\subsection{Properties of the Host Stars}

The stellar properties were derived from high-resolution spectroscopy and (for 11 KOIs) from asteroseismology, providing accurate values of stellar mass, radius, metallicity, and age (see Table 1 and Section 3). The 22 host stars have effective temperatures in the range, $T_{\text {eff }}=4700-6300 \mathrm{~K}$, i.e., mid-K-type stars to late $\mathrm{F}$ stars. The surface gravities are in the range, $\log g=$ 4.1-4.6, i.e., on the main sequence. The 22 metallicities are solar within 0.3 dex, with comparable numbers of slightly metal-rich and metal-poor stars, similar to the solar neighborhood. The ages of the stars (from stellar structure models) are distributed between 1-8.5 Gyr, with one (Kepler-25) nominally 11 Gyr old, consistent with the distribution of ages in the solar neighborhood. Thus, the $22 \mathrm{KOIs}$ in the stellar sample presented here are apparently similar to the distribution of FGK stars in the solar neighborhood.

\subsection{Properties of the Planets and Host Stars}

The planet properties were derived from modeling the transit light curve and the RVs, yielding measurements of the planet radii, masses, and bulk densities for the 42 transiting planets, listed in Table 2 and described in Section 5. We found 16 transiting planets that have masses and densities measured at a confidence level of $2 \sigma$ or better, useful for characterizing the population of the planets statistically. Another 14 planets emerged having uncertainties in density less than $6.5 \mathrm{~g} \mathrm{~cm}^{-3}$, offering constraints on the light element content.

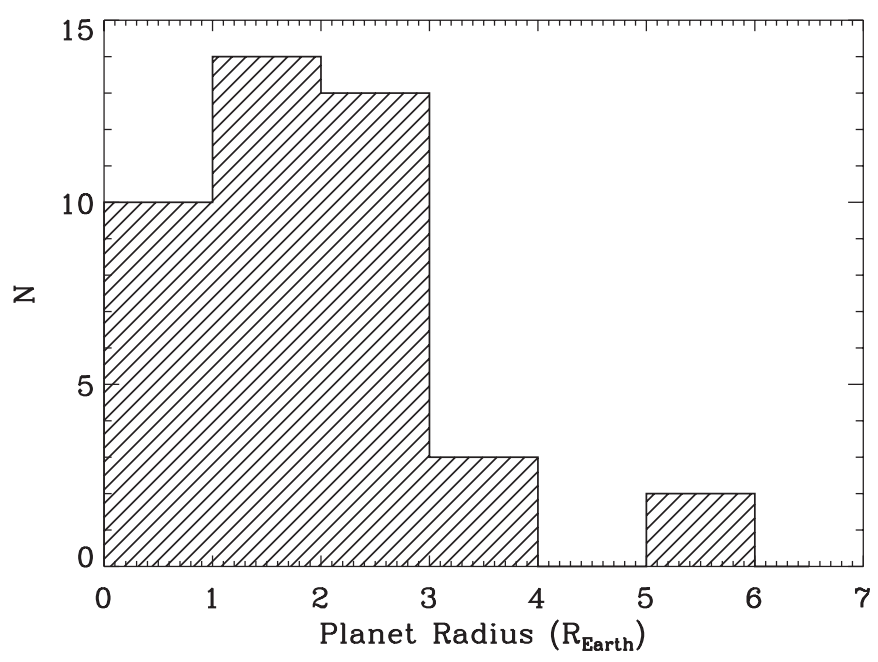

Figure 45. Histogram of the radii of the 42 transiting planets studied here, most of which are smaller than $3 R_{\oplus}$, with 6 greater than $3 R_{\oplus}$. The radii were determined from full models of the transit light curves, and stellar radii coming from analysis of high-resolution spectra and, for 11 cases, asteroseismological analysis.

Figure 45 shows a histogram of the 42 planet radii in the present sample of transiting planets. By selection, the radii are mostly smaller than $4 R_{\oplus}$ with most being $1-3 R_{\oplus}$. This range of planet radii corresponds to the most common size of planets (above $1 R_{\oplus}$ ) revealed by Kepler (Howard et al. 2012; Fressin et al. 2013; Petigura et al. 2013). The 22 KOIs, harboring 42 planets, were selected by the radii of the planet candidates, favoring 1-3 $R_{\oplus}$, without knowledge of their masses or densities.

Figure 46 shows the histogram of 42 planet masses measured here. The mass of each planet is defined arbitrarily as the peak mass (mode) of the posterior distribution of masses from the MCMC analysis and planet-model of the light curve and RVs. The planet masses span a range up to $25 M_{\oplus}$. Four planets have a peak in the posterior distribution at negative mass, due to errors in the RVs or "noise" from other planets (see Section 5), and four planets have densities unphysically high, namely Kepler102b, Kepler-37c, Kepler-37b, Kepler-103c, due to long orbital periods.

Figure 47 shows the histogram of planet densities for the 42 transiting planets. The densities span a range from near zero to $15 \mathrm{~g} \mathrm{~cm}^{-3}$. Planets with low density are presumably composed of considerable amounts of $\mathrm{H}$ and $\mathrm{He}$ gas (by volume) and planets with densities above $6 \mathrm{~g} \mathrm{~cm}^{-3}$ are likely composed mostly of rock and iron-nickel (Batygin \& Stevenson 2013; Zeng \& Seager 2008; Rogers et al. 2011; Valencia et al. 2007). While negative mass and negative density are not physical, bulk densities of $10-12 \mathrm{~g} \mathrm{~cm}^{-3}$ may be composed of solid or compressed iron. Several planets have densities out of the range of the plot due to poorly determined masses (See Table 2).

Figure 48 shows planet mass as a function of planet radius, for the 16 planets having a mass measured here at the $2 \sigma$ level or better. The planet masses represent the peak of the posterior mass distribution, and the uncertainties represent the mass $34 \%$ in area from the peak in both directions. Planet mass is correlated with radius, but the available radii and masses and their scatter warrant only a linear fit. A weighted linear fit (dotted line) has the relation: $M=3.28 R+0.79$ with $\mathrm{M}$ and $\mathrm{R}$ representing the planet mass and radius in Earth units. The scatter of the masses about the linear fit is roughly consistent with the uncertainties 


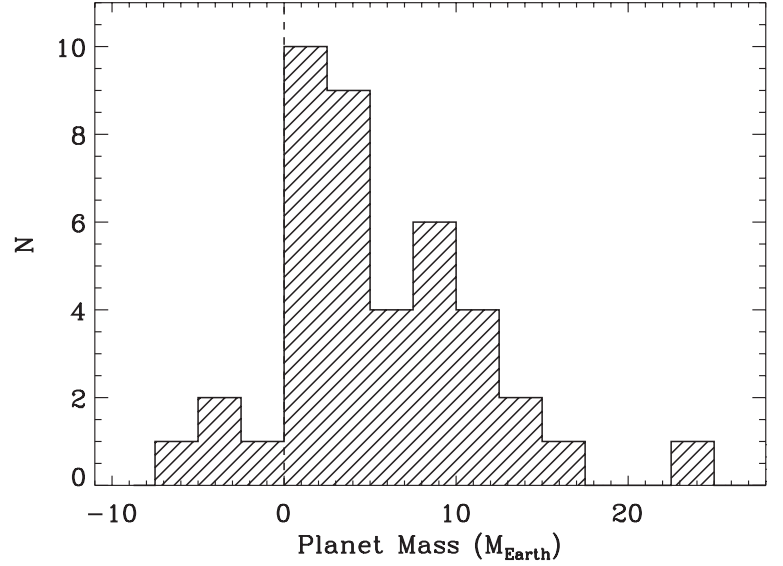

Figure 46. Histogram of the masses of the 42 transiting planets studied here. The mass determinations stem from the multiple RV measurements of the host star and the constrained orbital period and measured time of transit from the Kepler photometry. Circular orbits were assumed in all cases. Mass determinations represent the peak of the posterior mass distribution from the MCMC analysis of the RVs. For four planets, fluctuations (errors) in the RVs happen to yield a peak in the posterior mass distribution that resides at a negative mass ( $K$ is negative, given the frozen orbital phase). While unphysical, the negative masses offer an unbiased statistical balance against the fluctuations that yield overly positive masses. The masses span a range up to $25 M_{\oplus}$. See Figure 49 for the well determined planet masses.

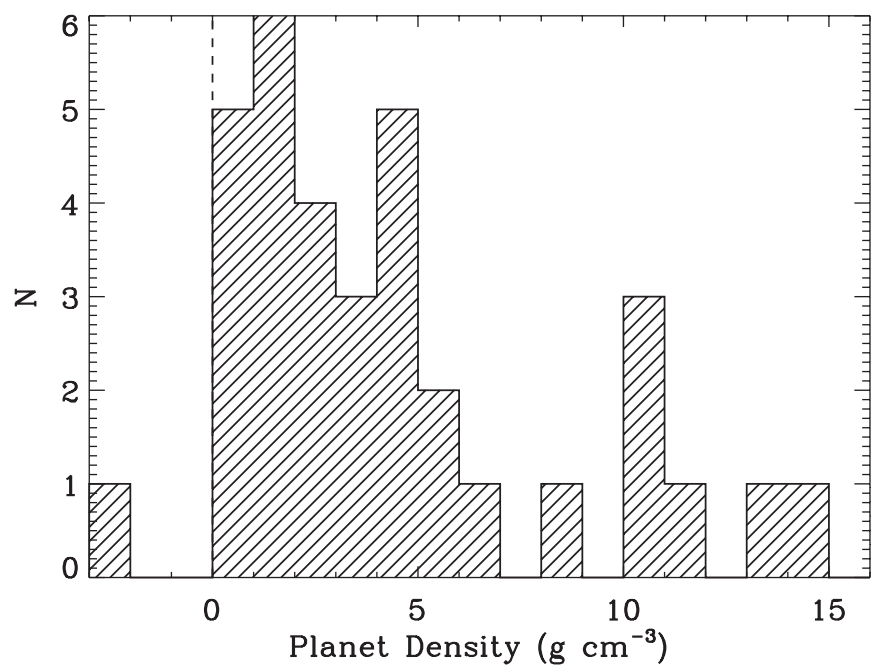

Figure 47. Histogram of the densities of the 42 transiting planets studied here. Four values of density are negative, due to negative masses (See Figure 46), three being offscale. The physical planet densities span a range from below unity to $15 \mathrm{~g} \mathrm{~cm}^{-3}$. As each density is the mode of a broad posterior distribution, caution should be exercised in interpreting the histogram. See Figure 51 for the well-measured density measurements.

(error bars), leaving unclear whether some of the scatter in mass at a given radius is intrinsic to the planets. For radii above $4 R_{\oplus}$, the mass-radius relation curves upward, as is easily seen by placing gas giant planets on the plot at $R \approx 11 R_{\oplus}$ and $M=$ $318 M_{\oplus}$. Two companion papers will examine this relationship more closely (Weiss \& Marcy (2013) and L. Rogers et al. 2014, in preparation).

Figure 49 shows planet mass versus planet radius for the 16 planets with $2 \sigma$ mass detections, as in Figure 48. Here, we include the 14 additional planets that do not meet the $2 \sigma$ criterion, i.e., have a poorer mass determination. For them, we plot only an upper limit as a downward arrow. The upper limit is defined to be the planet mass at the 95th percentile of the posterior distribution. Including the upper limits provides 14

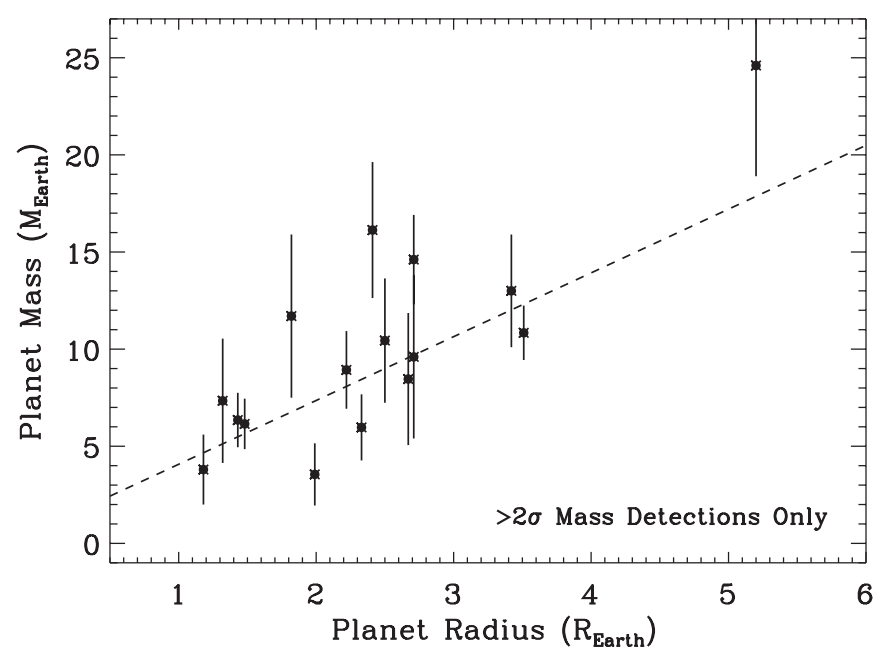

Figure 48. Planet mass vs. radius for the 16 transiting planets studied here having a mass measured at the $2 \sigma$ confidence level or better. Planet mass increases systematically with increasing radius. The weighted, linear fit to the planet masses and radii is, $M=3.28 R+0.79$ (with $M$ and $R$ in Earth units), for planet radii in the range $1-5.5 R_{\oplus}$. The monotonic relationship is consistent with a predominantly rocky composition for planets smaller than $\sim 2 R_{\oplus}$, and an increasing contribution of low-density material (probably $\mathrm{H}, \mathrm{He}$, or light molecules) for larger planets. The present data do not warrant more than a linear relationship, but see Weiss \& Marcy (2013) and L. Rogers et al. (2014, in preparation).

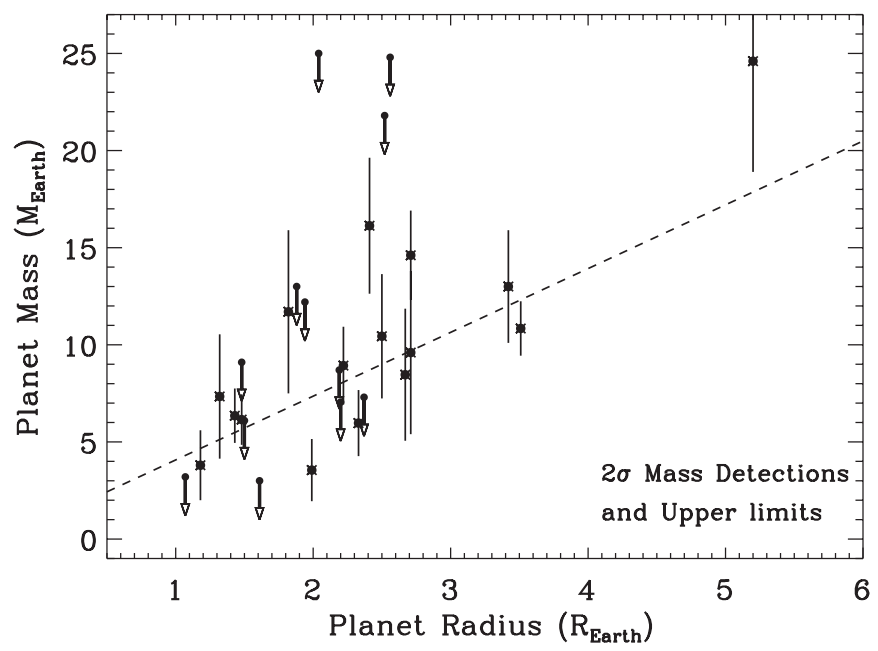

Figure 49. Planet mass vs. radius for both the 16 transiting planets having a $2 \sigma$ mass measurement, along with 14 upper limits to the masses of the remaining planets. The best-fit linear relation between planet mass and radius is, $M=3.28 R+0.79$ (with $M$ and $R$ in Earth units), for planet radii in the range $1-5.5 R_{\oplus}$. The upper limits are consistent with the linear trend in M vs. R found from the 16 detections and their scatter. These upper limits are consistent with mass increasing systematically with radius, as in Figure 48 Some planets appear to have masses below the linear fit at the $2 \sigma$ level, indicating that some of them contain more volatile material than average for their radius, i.e., diversity of composition for a given radius. See Weiss \& Marcy (2013) and L. Rogers et al. (2014, in preparation) for further statistical treatment. The 12 planets with an uncertainty in density greater than $6.5 \mathrm{~g} \mathrm{~cm}^{-3}$ were excluded from this plot.

additional masses and radii, which are apparently consistent with the linear fit to the 16 planets with $2 \sigma$ masses. Two upper limits in mass reside below the linear fit, but not by more than the errors. Thus the monotonic linear relation between mass and radius is not refuted by inclusion of the upper limits in planet mass. We exclude from this figure the 12 planets having mass determinations so poor that the density is uncertain by more than $6.5 \mathrm{~g} \mathrm{~cm}^{-3}$. 


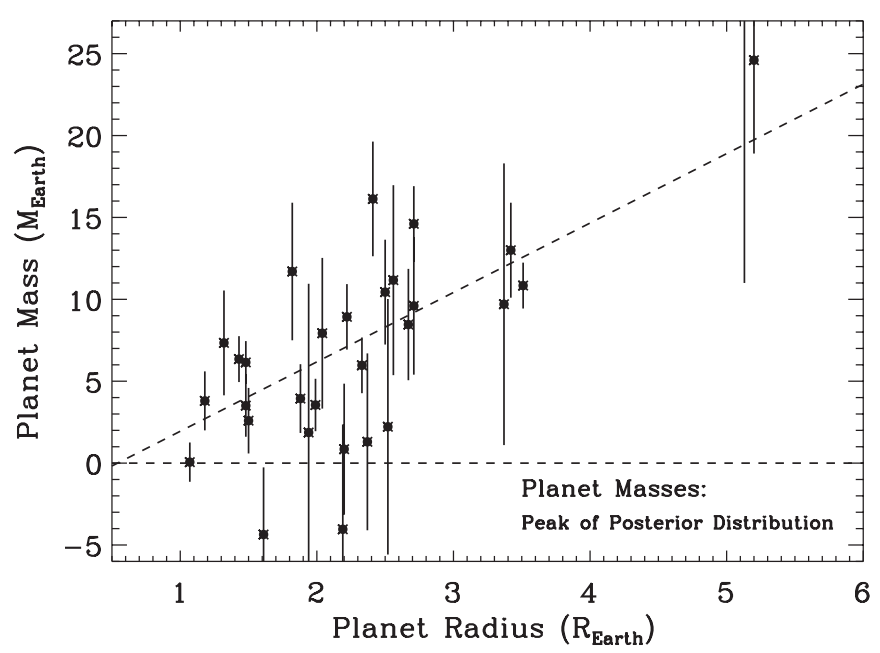

Figure 50. Planet mass vs. radius for 30 transiting planets measured here. The plotted masses represent the peak of the posterior distribution of the planet masses. Most peaks reside at positive masses, but RV fluctuations occasionally yield a peak at negative masses, clearly representing non-detections. We retain the negative masses to avoid Lutz-Kelker-type bias toward positive masses. The best-fit linear relation is $M=4.24 R-2.29$ (in Earth units) for planet radii in the range $1-5.5 R_{\oplus}$, including negative masses in the fit. Of the 42 transiting planets studied here, we excluded from this plot the 12 transiting planets having a density uncertainty greater than $6.5 \mathrm{~g} \mathrm{~cm}^{-3}$ (but included all mass measurements, even if less than a $2 \sigma$ detection).

Figure 50 shows planet mass versus planet radius for all 30 transiting planets. (We exclude the 12 planets with uncertainties in density greater than $6.5 \mathrm{~g} \mathrm{~cm}^{-3}$.) Planet mass increases monotonically with radius, as seen in Figure 49. Here, masses less secure than $2 \sigma$, and even negative masses, are included in the plot. We specifically allowed our MCMC analysis to include negative planet masses in the solution. Among the 42 transiting planets, 4 of them had a peak in the posterior mass distribution that was negative (see Table 2). Such unphysical masses are a normal outcome of fluctuations in the RV measurements from both RV errors and unknown planets, which will be mostly uncorrelated in orbital phase with the known planets, aside from mean motion resonances. Negative masses arise naturally from $\mathrm{RV}$ uncertainties of $\mathrm{m} \mathrm{s}^{-1}$ for planets that induce RV semiamplitudes, $K$, that are smaller (due to small planet mass and/or long period). RV errors may cause measured RVs to be high when the actual RV is low, and vice versa, due to those errors. For the low mass planets, such RV non-detections are expected, yielding equal numbers of apparently positive and "negative" masses.

The "negative" masses are useful for any statistical treatment of masses or densities of the planet population as an ensemble. Because planet masses are certainly positive, the ensemble of planets (in a given range of planet radii) will, on average, yield positive values of mass. As the KOI target selection was based on planet radius not mass, we may measure the average planet mass as a function of planet radius. We report in Table 2, Column 5, the planet masses corresponding to the peak in the MCMC posterior distribution, four of which are negative. For the uncertainty, we report the mass corresponding to $34 \%$ of the integrated area of the distribution on either side, representing the $1 \sigma$ departure from the peak mass.

Figure 51 shows planet density versus radius for the 30 transiting planets having an uncertainty in density less than $6.5 \mathrm{~g} \mathrm{~cm}^{-3}$. Planet density decreases with increasing planet radius from 1.5 to $5 R_{\oplus}$. The densities of planets smaller than

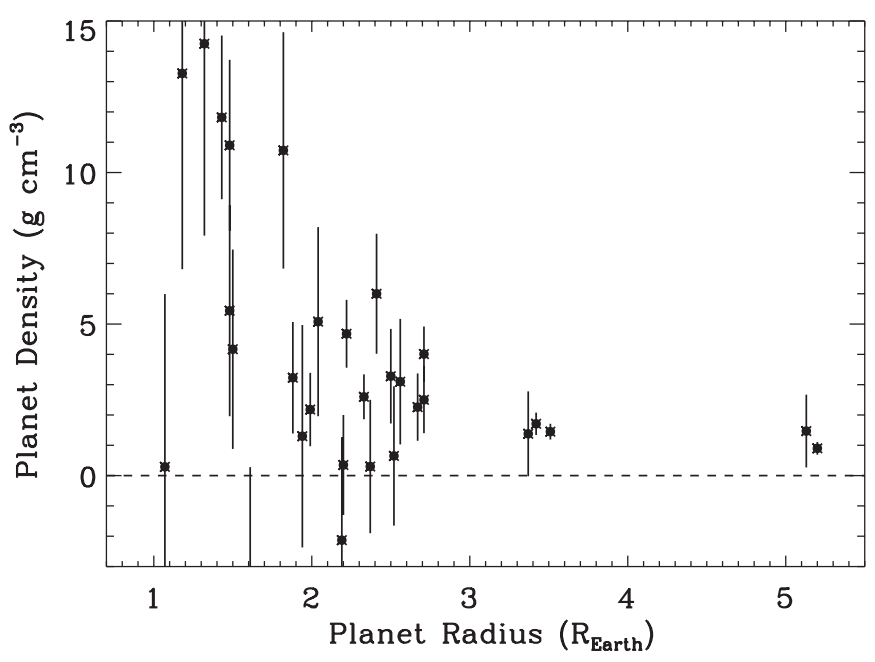

Figure 51. Planet density vs. radius for all 30 transiting planets having an uncertainty in density less than $6.5 \mathrm{~g} \mathrm{~cm}^{-3}$. Planet density declines systematically with increasing planet radius in the domain of $1.0-5.5 R_{\oplus}$. Increasing planet radius apparently is associated with increasing amounts of low-density material, presumably $\mathrm{H}, \mathrm{He}$, and light molecules. For an analysis of density vs. radius for all known planets, see Weiss \& Marcy (2013) and L. Rogers et al. 2014 (in preparation).

$1.5 R_{\oplus}$ are systematically greater than $\sim 5 \mathrm{~g} \mathrm{~cm}^{-3}$, similar to that of Earth $\left(5.5 \mathrm{~g} \mathrm{~cm}^{-3}\right)$, indicating that these planets have mostly rocky interiors. Planets with radii larger than $2 R_{\oplus}$ have densities predominatly and increasingly less than $5 \mathrm{~g} \mathrm{~cm}^{-3}$, indicating increasing amounts by volume of light material, presumably $\mathrm{H}$ and He (Batygin \& Stevenson 2013; Zeng \& Seager 2008; Rogers et al. 2011; Valencia et al. 2007). Thus the transition from rocky to gas-dominated planets (by volume) occurs at roughly $2 R_{\oplus}$ (Weiss \& Marcy (2013), and L. Rogers et al. (2014, in preparation).

Figure 52 shows planet density versus planet mass for 30 transiting planets measured here. Planet density is not a strong function of planet mass in the domain of $0-30 M_{\oplus}$. There is weak evidence in the plot of a rise in density for masses from $0-6 M_{\oplus}$, and a decline in density for planet masses 6-25 $M_{\oplus}$. This behavior of the density is consistent with increasing gravitational compression of rocky planet interiors with increasing mass from $1-6 M_{\oplus}$. The decline in density from 6-25 $M_{\oplus}$ is consistent with an increasing contribution from light elements, $\mathrm{H}$ and $\mathrm{He}$, toward Neptune-like envelopes.

Figure 53 shows planet radius versus orbital period for 30 well-measured transiting planets. The planet radii are not a strong function of orbital period. It is difficult to interpret this lack of correlation securely. Selection effects may contribute to the sizes, and hence anticipated masses, of planets farther from the host star where Doppler reflex velocities are lower. Figure 54 shows planet density versus orbital period for 30 well-measured transiting planets, which shows no clear dependence. As with Figure 53, the interpretation of no correlation between period and bulk density is not clear, due to possible selection effects with orbital period.

Figure 55 shows a histogram of impact parameters, $b$, for all 42 transiting planets. The impact parameter is mostly decoupled from the RV and mass detections. The values of $b$ offer a test of the integrity of the light curve fitting. The roughly uniform distribution of $b$ from $0-1$ is consistent with the expectation of randomly oriented orbital planes leading to a uniform distribution of impact parameters, $b$. We note that the 


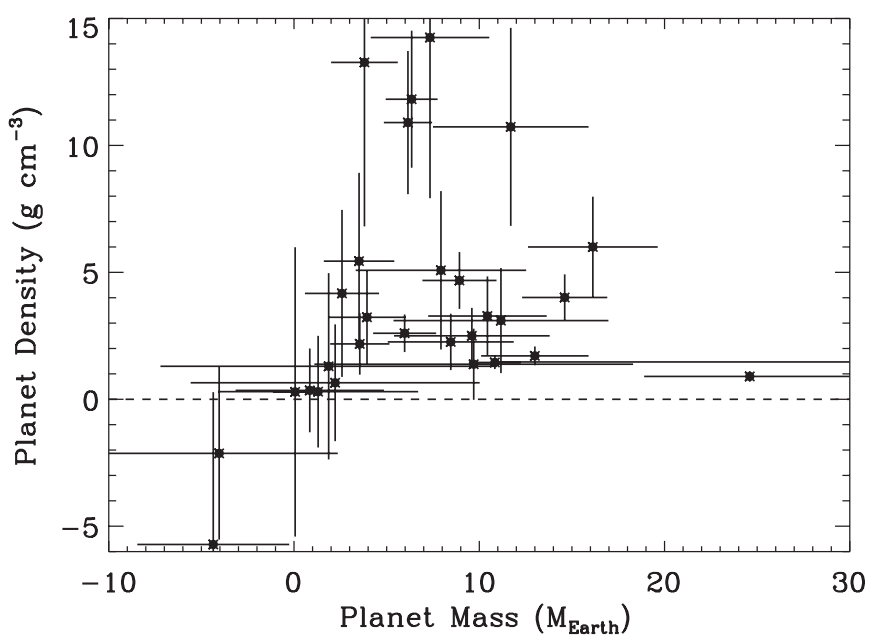

Figure 52. Planet density vs. planet mass for the 30 transiting planets measured here. Planet density appears to increase from 1 to $\sim 6 M_{\oplus}$, and then decline from $\sim 6-25 R_{\oplus}$, with large scatter in density. This behavior is consistent with planets of 0-6 $M_{\oplus}$ having a mostly rocky interior experiencing increased gravitational compression (or greater iron/nickel content). The decline in density for planet masses, $M>6 M_{\oplus}$ indicates increasing amounts of low-density material, presumably $\mathrm{H}, \mathrm{He}$, and light molecules, with increasing planet mass. The scatter in planet density, at a given planet mass, indicates a diversity of chemical composition at a given mass, with different admixtures of iron, rock, and light elements.

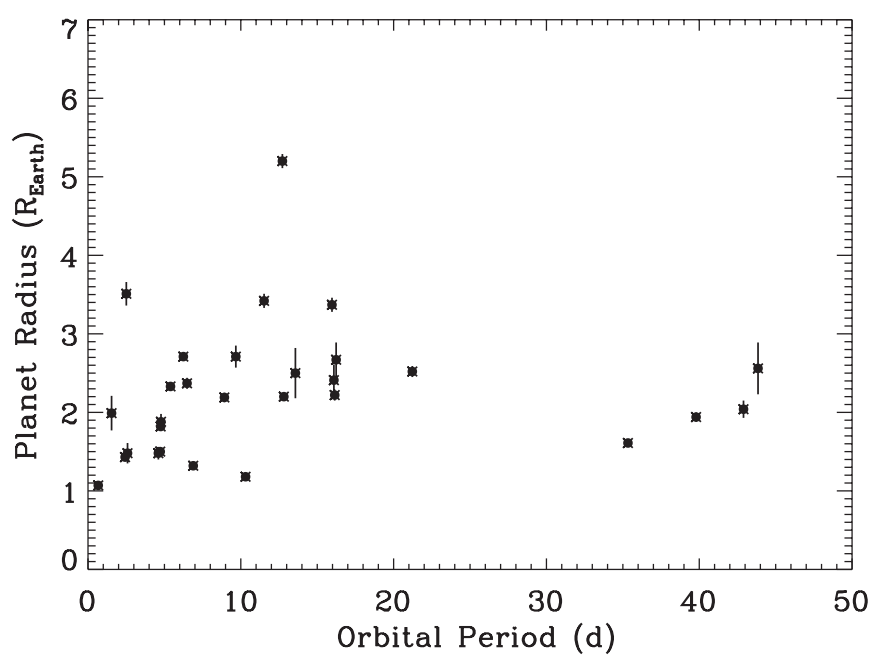

Figure 53. Planet radius vs. orbital period for 30 well-measured transiting planets. Planet radii are not strongly correlated with orbital period in the present sample.

impact parameter distribution in Batalha et al. (2013) appears not to be uniform, raising concerns about bias there.

\subsection{Conclusions about Planet Composition and Formation}

In general, the distribution of planet masses for a given planet radius may be a function of orbital period and the type of host star, stemming from the complex processes of planet formation in a protoplanetary disk. The distribution of planet masses surely depends on planet radius, stellar mass, orbital semi-major axis and eccentricity, and on the chemical and thermodynamic properties of the protoplanetary region where they form. Thus, the measured planet masses and radii here inform only one plane of a multi-dimensional space that characterizes planet properties.

Planet density may decrease with increasing planet radius due to an increasing admixture of light building material $(\mathrm{H}, \mathrm{He}$, and

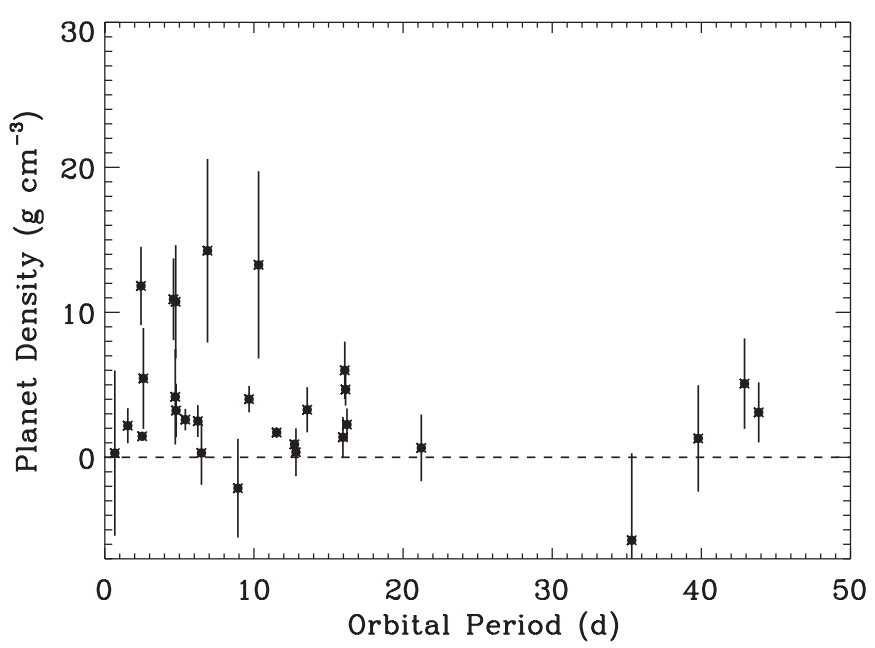

Figure 54. Planet density vs. orbital period for 30 well-measured transiting planets. Planet density is not strongly correlated with orbital period. There is no convincing evidence that the density, and hence planet composition, is a strong function of orbital distance, out to periods of 45 days. But mass detection biases may affect this interpretation.

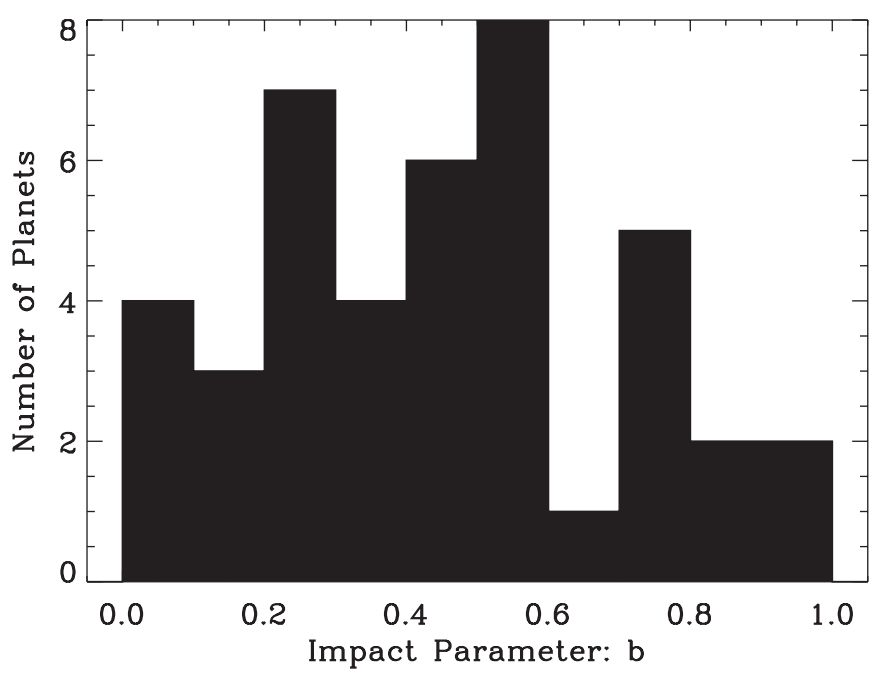

Figure 55. Histogram of impact parameters, $b$, for all 42 transiting planets studied here. The impact parameter is derived from a self-consistent transitingplanet model fit to the photometry and RVs, with constraints on the stellar radius from spectroscopy and asteroseismology. The roughly uniform distribution of impact parameters, from 0 to 1 , is consistent with the geometrical expectation of a random distribution of orbital tilt angles relative to our line of sight, leading to a uniform distribution of impact parameters, $b$.

water) (Lopez et al. 2012; Lopez \& Fortney 2013; Rogers et al. 2011; Rogers \& Seager 2010b; Zeng \& Seager 2008; Weiss \& Marcy 2013) and see also L. Rogers et al. (2013, in preparation). Indeed this decline in bulk density with increasing radius is seen clearly in Figure 51. This supports previous analyses, e.g., Weiss et al. (2013), that similarly noted a declining density with increasing radius. This density behavior with radius strongly supports the suggestion that planets with sizes increasing above $2 R_{\oplus}$ have interiors with an increasing contribution from light elements, $\mathrm{H}$ and $\mathrm{He}$, as described theoretically by (Batygin \& Stevenson 2013; Zeng \& Seager 2008; Rogers et al. 2011; Valencia et al. 2007).

Interestingly, average planet masses may remain constant or even decrease with increasing planet radii for radii near the transition from rocky to volatile-rich planets near $2 R_{\oplus}$. For example, planets of $1.8 R_{\oplus}$ may, on average, be only slightly 
more massive (or even less massive) than those of $1.5 R_{\oplus}$ if they contain large differences in the amount of light elements.

The unknown composition and density of planets having radii, $R<3 R_{\oplus}$ motivated the RV measurements here, but left great uncertainty about whether RV signals would be detectable. In retrospect, the low densities near $1 \mathrm{~g} \mathrm{~cm}^{-3}$ for the planets with $R>2.5 R_{\oplus}$ certainly led to many of the non-detections of RV signals we found. We simply didn't know, at the start, if $\sim 2.5 R_{\oplus}$ planets were mostly rocky or mostly volatile-rich. It is worth emphasizing that for each planet candidate only the radius was known, leaving the resulting mass and density unbiased for the given planet radius.

The primary goal of this paper is to provide a statistically useful and unbiased set of planet masses and densities as a function of planet radii. However, these planet masses and densities pertain only to the stellar masses of $0.8-1.1 M_{\odot}$ and to orbital periods $(P<25 \mathrm{~d})$ represented in this work. The population of planet masses and densities, as a function of radius, may be a function of orbital period and stellar mass, confining the conclusions here to such stars and orbital distances.

This work benefited greatly from the transit ephemeris for each candidate that came directly from the Kepler photometry. That ephemeris provided a prediction of the times (i.e., orbital phase) when the RVs were expected to be maxima or minima. We optimized the times of observations based on those predictions, to more efficiently detect and measure the RV semiamplitude, $K$, and hence planet mass. Moreover, for circular orbits, only the RV amplitude is unknown prior to taking RV measurements, thus concentrating the RV information into the measurement of planet mass, rather than having to constrain other orbital parameters too. The only caveat stems from the unknown orbital eccentricities.

Why were no clear false positives detected? This program began by studying 42 transiting planet "candidates." The Kepler photometry, planet-detection pipeline, and associated DV efforts conferred "KOI" status to these planet "candidates." The Kepler Follow-up Observation Team (FOP) had obtained low signalto-noise spectra for each of the $22 \mathrm{KOIs}$, rendering the stars and prospective planets suitable for precise RV follow-up. The FPPs were not known for the 22 KOIs and their associated 42 transiting planets. However we carried out careful vetting of the planets by detailed light curve models, astrometric "centroid" analysis, multiple high-resolution imaging, and sophisticated modeling of the FPP (Table 3). After all that, none of the 42 transiting planets were revealed to be false positives (but one has an FPP of $\sim 2 \%$ ).

This absence of false positives is noteworthy, as Fressin et al. (2013) and Morton (2012) predict an FPP of $\sim 10 \%$. We might have expected 4 false positives from 42 transiting Kepler planets, but we found none. The implication is that the preliminary recon spectroscopy, seeing-limited imaging, and the centroid analysis were effective in diminishing the false-positive rate. These KOIs are also much brighter than the typical KOI, these being magnitude 10-13 mag, and hence are closer than the typical Kepler KOI. Typical distances are only 100-200 pc, making the various imaging techniques more efficient at detecting angularly nearby stars that can harbor the false positive. The Kepler pipeline and DV efforts seem to be performing well in keeping the false-positive rate of small planets to no more than the $10 \%$ rate that one predicts simplistically without imaging and spectroscopy. Moreover, many of the KOIs studied here are multi-planet transiting systems which have been shown to be mostly real planets (Lissauer et al. 2012). Based on these 42 transiting planets, the Kepler pipeline and vetting appears to have a false-positive rate under $10 \%$ for planets less than $4 R_{\oplus}$. This conclusion deserves a more careful analysis, including quantitative assessment of early vetting of these candidates.

\subsection{Rocky Planets and Interiors}

Six of the planets studied here have $2 \sigma$ densities between $5.0-12.0 \mathrm{~g} \mathrm{~cm}^{-3}$. They are marked in bold in Column 7 of Table 2. These six planets are likely to have compositions that consist mostly of rocky or iron/nickel material. Those six rocky planets are Kepler-100b, Kepler-102e, Kepler-102d, Kepler48c, Kepler-113b, Kepler-131b, Kepler-99b, and Kepler-406b. Most of these planets have densities secure only at the $2 \sigma-3 \sigma$ level. They should be considered only "candidate" rocky planets, requiring further RVs to secure their masses and densities.

Several of these six high-density planets have densities greater than that of Earth, making them plausibly composed nearly entirely of rock or iron/nickel. These planets join previously detected planets that have measured masses and radii implying rocky compositions, namely CoRoT-7b, Kepler-10b and Kepler-36b.

Detailed models of planet interiors, including possible chemical compositions, stratified differentiation, and equations of state are needed to predict the plausible bulk densities associated with planets with a given mass. Recent work on the interiors of rocky planets have been carried out by Rogers \& Seager (2010a, 2010b), Rogers et al. (2011), Zeng \& Sasselov (2013), Lopez \& Fortney (2013), and L. Rogers et al. (2014, in preparation).

Discussions have ensued about whether iron-rich planets such as Mercury, might form from giant collisions that strip the silicate and volatile envelopes or instead whether such compositional oddities might result from processes intrinsic to planet formation. Recent work by Wurm et al. (2013) provides an interesting mechanism of photophoretic separation of metals from silicates. Meanwhile, models of photo-evaporation seem promising in predicting the statistical behavior of these close-in rocky planets (Lopez et al. 2012; Lopez \& Fortney 2013).

Future observations of RVs and TTVs are needed to provide more masses and densities for small planets. It is highly desirable to measure masses and densities for $1-3 R_{\oplus}$ planets that orbit farther from the host star, to determine whether the transition size between rocky and gaseous planets is different at 1 au than at 0.1 au as measured here.

Kepler was competitively selected as the tenth NASA Discovery mission. Funding for this mission is provided by the NASA Science Mission Directorate. Some of the data presented herein were obtained at the W. M. Keck Observatory, which is operated as a scientific partnership among the California Institute of Technology, the University of California, and the National Aeronautics and Space Administration. The Keck Observatory was made possible by the generous financial support of the W. M. Keck Foundation. Some of the asteroseismology analysis was performed by the Stellar Astrophysics Centre which is funded by the Danish National Research Foundation (Grant DNRF106). The research is supported by the ASTERISK project (ASTERoseismic Investigations with SONG and Kepler) funded by the European Research Council (Grant 267864). D.H. is supported by an appointment to the NASA Postdoctoral Program at Ames Research Center, administered by Oak Ridge Associated Universities through a contract with NASA. W.J.C., Y.E., T.L.C., G.R.D, R.H and 
A.M. acknowledge financial support from the UK Science and Technology Facilities Council (STFC). S.B. acknowledges NSF grant AST-1105930. Funding for the Stellar Astrophysics Centre is provided by The Danish National Research Foundation (Grant agreement no.: DNRF106). The research is supported by the ASTERISK project (ASTERoseismic Investigations with SONG and Kepler) funded by the European Research Council (Grant agreement no. 267864). S.H. acknowledges financial support from the Netherlands organisation for Scientific Research (NWO). The research leading to the presented results has received funding from the European Research Council under the European Community's Seventh Framewrok Programme (FP7/2007-2013)/ERC grant agreement no. 338251 (StellarAges). W. F. Welsh and J. A. Orosz acknowledge support from NASA through the Kepler Participating Scientist Program and from the NSF via grant AST-1109928. D. Fischer acknowledges support from NASA ADAP12-0172. O. R. Sanchis-Ojeda \& J. N. Winn are supported by the Kepler Participating Scientist Program (PSP) through grant NNX12AC76G. E. Ford is partially supported by NASA PSP grants NNX08AR04G \& NNX12AF73G. Eric Agol acknowledges NSF Career grant AST-0645416. R.L.G. has been partially supported by NASA co-operative agreement: NNX09AG09A. A. Santerne acknowledges the support by the European Research Council/European Community under the FP7 through Starting Grant agreement number 239953. The authors would like to thank the many people who gave so generously of their time to make this Mission a success. All Kepler data products are available to the public at the Mikulski Archive for Space Telescopes http://stdatu.stsci.edu/kepler and the spectra and their products are made available at the NExSci Exoplanet Archive and its CFOP Web site: http://exoplanetarchive.ipac.caltech.edu. We thank the many observers who contributed to the measurements reported here. We gratefully acknowledge the efforts and dedication of the Keck Observatory staff, especially Scott Dahm, Hien Tran, and Grant Hill for support with HIRES and Greg Wirth for support with remote observing. This work made use of the SIMBAD database (operated at CDS, Strasbourg, France) and NASA's Astrophysics Data System Bibliographic Services. This research has made use of the NASA Exoplanet Archive, which is operated by the California Institute of Technology, under contract with the National Aeronautics and Space Administration under the Exoplanet Exploration Program. Finally, the authors wish to extend special thanks to those of Hawai 'ian ancestry on whose sacred mountain of Mauna Kea we are privileged to be guests. Without their generous hospitality, the Keck observations presented herein would not have been possible.

\section{REFERENCES}

Adams, E. R., Ciardi, D. R., Dupree, A. K., et al. 2012, AJ, 144, 42 Albrecht, S., Winn, J. N., Marcy, G. W., et al. 2013, ApJ, 711, 11 Argabright, V. S., VanCleve, J. E., Bachtell, E. E., et al. 2008, Proc. SPIE, 7010, 70102L

Baranne, A., Queloz, D., Mayor, M., et al. 1996, A\&AS, 119, 373 Barclay, T., Rowe, J. F., Lissauer, J. J., et al. 2013, Natur, 494, 452 Batalha, N. M., Borucki, W. J., Bryson, S. T., et al. 2011, ApJ, 729, 27 Batalha, N. M., Rowe, J. F., Bryson, S. T., et al. 2013, ApJS, 204, 24 Batalha, N. M., Rowe, J. F., Gilliland, R. L., et al. 2010, ApJL, 713, L103

Batygin, K., \& Stevenson, D. J. 2013, ApJL, 769, L9

Boisse, I., Eggenberger, A., Santos, N. C., et al. 2010, A\&A, 523, A88

Bonfils, X., Gillon, M., Udry, S., et al. 2012, A\&A, 546, A27

Borucki, W. J., Agol, E., Fressin, F., et al. 2013, Sci, 340, 587

Borucki, W. J., Koch, D., Basri, G., et al. 2010, Sci, 327, 977

Borucki, W. J., Koch, D. G., Basri, G., et al. 2011, ApJ, 736, 19
Borucki, W. J., Koch, D. G., Batalha, N., et al. 2012, ApJ, 745, 120

Bouchy, F., Hébrard, G., Udry, S., et al. 2009, A\&A, 505, 853

Bruntt, H., Basu, S., Smalley, B., et al. 2012, MNRAS, 423, 122

Bryson, S. T., Jenkins, J. M., Gilliland, R. L., et al. 2013, PASP, 125, 889

Buchhave, L. A., Latham, D. W., Carter, J. A., et al. 2011, ApJS, 197, 3

Buchhave, L. A., Latham, D. W., Johansen, A., et al. 2012, Natur, 486, 375

Caldwell, D. A., Kolodziejczak, J. J., Van Cleve, J. E., et al. 2010, ApJL, 713, L92

Carter, J. A., Agol, E., Chaplin, W. J., et al. 2012, Sci, 337, 556

Charbonneau, D., Berta, Z. K., Irwin, J., et al. 2009, Natur, 462, 891

Chiang, E., \& Laughlin, G. 2013, MNRAS, 431, 3444

Chubak, C., Marcy, G., Fischer, D. A., et al. 2012, arXiv:1207.6212

Claret, A., \& Bloemen, S. 2011, A\&A, 529, A75

Cochran, W. D., Fabrycky, D. C., Torres, G., et al. 2011, ApJS, 197, 7

Demarque, P., Woo, J., Kim, Y., \& Yi, S. K. 2004, ApJS, 155, 667

Demory, B.-O., de Wit, J., Lewis, N., et al. 2013, ApJL, 776, L25

Demory, B.-O., Gillon, M., Deming, D., et al. 2011, A\&A, 533, A114

Désert, J. M. 2014, submitted

Dressing, C. D., \& Charbonneau, D. 2013, ApJ, 767, 95

Endl, M., Robertson, P., Cochran, W. D., et al. 2012, ApJ, 759, 19

Fabrycky, D. C., Lissauer, J. J., Ragozzine, D., et al. 2012, arXiv:1202.6328

Figueira, P., Pont, F., Mordasini, C., et al. 2009, A\&A, 493, 671

Fischer, D. A., \& Valenti, J. 2005, ApJ, 622, 1102

Fortney, J. J., Marley, M. S., \& Barnes, J. W. 2007, ApJ, 659, 1661

Fressin, F., Torres, G., Charbonneau, D., et al. 2013, ApJ, 766, 81

Gautier, T. N., III, Batalha, N. M., Borucki, W. J., et al. 2010, arXiv:1001.0352

Gilliland, R. L., Marcy, G. W., Rowe, J. F., et al. 2013, ApJ, 766, 40

Gillon, M., Pont, F., Demory, B.-O., et al. 2007, A\&A, 472, L13

Goldreich, P., Lithwick, Y., \& Sari, R. 2004, ARA\&A, 42, 549

Hansen, B. M. S., \& Murray, N. 2013, ApJ, 775, 53

Hartman, J. D., Bakos, G. ÁÁ., Kipping, D. M., et al. 2011, ApJ, 728, 138

Hayward, T. L., Brandl, B., Pirger, B., et al. 2001, PASP, 113, 105

Hébrard, G., Bouchy, F., Pont, F., et al. 2008, A\&A, 488, 763

Hirano, T., Sanchis-Ojeda, R., Takeda, Y., et al. 2012, ApJ, 756, 66

Horch, E. P., Veillette, D. R., Baena Gallé, R., et al. 2009, AJ, 137, 5057

Hormuth, F. 2007, Master's thesis, Univ. Heidelberg

Howard, A. W., Marcy, G. W., Bryson, S. T., et al. 2012, ApJS, 201, 15

Howard, A. W., Marcy, G. W., Johnson, J. A., et al. 2010, Sci, 330, 653

Howard, A. W., Sanchis-Ojeda, R., Marcy, G. W., et al. 2013, Natur, 503, 381

Howell, S. B., Everett, M. E., Sherry, W., Horch, E., \& Ciardi, D. R. 2011, AJ, 142,19

Huber, D., Chaplin, W. J., Christensen-Dalsgaard,, et al. 2013, ApJ, 767, 127

Ida, S., \& Lin, D. N. C. 2010, ApJ, 719, 810

Isaacson, H., \& Fischer, D. 2010, ApJ, 725, 875

Jenkins, J. M., Caldwell, D. A., Chandrasekaran, H., et al. 2010a, ApJL, 713, L120

Jenkins, J. M., Caldwell, D. A., Chandrasekaran, H., et al. 2010b, ApJL, 713, L87

Johansson, E. M., van Dam, M. A., Stomski, P. J., et al. 2008, Proc. SPIE, 7015, $70153 \mathrm{E}$

Johnson, J. A., Butler, R. P., Marcy, G. W., et al. 2007, ApJ, 670, 833

Johnson, J. A., Howard, A. W., Bowler, B. P., et al. 2010, PASP, 122, 701

Latham, D. W., \& Buchhave, L. A. 2012, in American Astronomical Society Meeting Abstracts, Vol. 220, 306.03

Lawrence, A., Warren, S. J., Almaini, O., et al. 2007, MNRAS, 379, 1599

Lillo-Box, J., Barrado, D., \& Bouy, H. 2012, A\&A, 546, A10

Lissauer, J. J., Marcy, G. W., Rowe, J. F., et al. 2012, ApJ, 750, 112

Lissauer, J. J., Ragozzine, D., Fabrycky, D. C., et al. 2011, ApJS, 197, 8

Lissauer, J. J., Marcy, G. W., Bryson, S. T., et al. 2014, ApJ, submitted

Lissauer, J. J., et al. 2013, ApJ, in press

Lithwick, Y., Xie, J., \& Wu, Y. 2012, ApJ, 761, 122

Lopez, E. D., \& Fortney, J. J. 2013, ApJ, 776, 2

Lopez, E. D., Fortney, J. J., \& Miller, N. 2012, ApJ, 761, 59

Mandel, K., \& Agol, E. 2002, ApJL, 580, L171

Maness, H. L., Marcy, G. W., Ford, E. B., et al. 2007, PASP, 119, 90

Marcy, G. W., \& Butler, R. P. 1992a, PASP, 104, 270

Marcy, G. W., \& Butler, R. P. 1992b, PASP, 104, 270

Marcy, G. W., Butler, R. P., Vogt, S. S., et al. 2001, ApJ, 555, 418

Marcy, G. W., Butler, R. P., Vogt, S. S., et al. 2008, PhST, 130, 014001

Mayor, M., Marmier, M., Lovis, C., et al. 2011, arXiv:1109.2497

Morbidelli, A. 2013, in Planets, Stars, and Stellar Systems, Vol. 3: Solar and

Stellar Planetary Systems, ed. T. D. Oswalt, L. M. French, \& P. Kalas, 63

Mordasini, C., Alibert, Y., Klahr, H., \& Henning, T. 2012, A\&A, 547, A111

Morton, T. D. 2012, ApJ, 761, 6

Morton, T. D., \& Johnson, J. A. 2011, ApJ, 738, 170

Muirhead, P. S., Hamren, K., Schlawin, E., et al. 2012, ApJL, 750, L37

Pepe, F., Cameron, A. C., Latham, D. W., et al. 2013, Natur, 503, 377 
Petigura, E., Marcy, G. W., \& Howard, A. 2013, in American Astronomical Society Meeting Abstracts, Vol. 221, 216.05

Pollack, J. B., Hubickyj, O., Bodenheimer, P., et al. 1996, Icar, 124, 62

Queloz, D., Bouchy, F., Moutou, C., et al. 2009, A\&A, 506, 303

Queloz, D., Henry, G. W., Sivan, J. P., et al. 2001, A\&A, 379, 279

Rappaport, S., Sanchis-Ojeda, R., Rogers, L. A., Levine, A., \& Winn, J. N. 2013, ApJL, 773, L15

Rogers, L. A., Bodenheimer, P., Lissauer, J. J., \& Seager, S. 2011, ApJ, 738, 59

Rogers, L. A., \& Seager, S. 2010a, ApJ, 712, 974

Rogers, L. A., \& Seager, S. 2010b, ApJ, 716, 1208

Rowe, J. F., Bryson, S. T., Marcy, G. W., et al. 2014, ApJ, submitted

Sanchis-Ojeda, R., Rappaport, S., Winn, J. N., et al. 2013, ApJ, 774, 54

Santerne, A., Díaz, R. F., Moutou, C., et al. 2012, A\&A, 545, A76

Seager, S., Bains, W., \& Hu, R. 2013, ApJ, 777, 95

Seager, S., Kuchner, M., Hier-Majumder, C. A., \& Militzer, B. 2007, ApJ, 669,1279

Steffen, J. H., Fabrycky, D. C., Agol, E., et al. 2013, MNRAS, 428, 1077

Steffen, J. H., Fabrycky, D. C., Ford, E. B., et al. 2012, MNRAS, 421, 2342

Torres, G., Fischer, D. A., Sozzetti, A., et al. 2012, ApJ, 757, 161

Torres, G., Fressin, F., Batalha, N. M., et al. 2011, ApJ, 727, 24

Torres, G., Winn, J. N., \& Holman, M. J. 2008, ApJ, 677, 1324
Troy, M., \& Chanan, G. 2003, ApOpt, 42, 3745

Valencia, D., Sasselov, D. D., \& O’Connell, R. J. 2007, ApJ, 665, 1413

Valenti, J. A., \& Fischer, D. A. 2005, ApJS, 159, 141

Valenti, J. A., \& Piskunov, N. 1996, A\&AS, 118, 595

Van Cleve, J. E., \& Caldwell, D. A. 2009, Kepler Instrument Handbook (KSCI19033)

Vogt, S. S., Allen, S. L., Bigelow, B. C., et al. 1994, Proc. SPIE, 2198, 362

Wang, J., Xie, J.-W., Barclay, T., \& Fischer, D. 2013, arXiv:1309.7097

Weiss, L. M., \& Marcy, G. W. 2013, arXiv:1312.0936

Weiss, L. M., Marcy, G. W., Rowe, J. F., et al. 2013, ApJ, 768, 14

Wizinowich, P. L., Le Mignant, D., Bouchez, A., et al. 2004, Proc. SPIE, 5490,1

Wu, H., Twicken, J. D., Tenenbaum, P., et al. 2010, Proc. SPIE, 7740, 774019

Wu, Y., \& Lithwick, Y. 2013, ApJ, 772, 74

Wurm, G., Trieloff, M., \& Rauer, H. 2013, ApJ, 769, 78

Xie, J.-W. 2013, ApJS, 208, 22

Yanny, B., Guhathakurta, P., Bahcall, J. N., \& Schneider, D. P. 1994, AJ, 107, 1745

Yi, S., Demarque, P., Kim, Y.-C., et al. 2001, ApJS, 136, 417

Zeng, L., \& Sasselov, D. D. 2013, arXiv e-prints

Zeng, L., \& Seager, S. 2008, PASP, 120, 983 